\title{
Dislocation dynamics simulations of plasticity at small scales
}

by

\author{
Caizhi Zhou \\ A dissertation submitted to the graduate faculty \\ in partial fulfillment of the requirements for the degree of \\ DOCTOR OF PHILOSOPHY \\ Major: Materials Science and Engineering \\ Program of Study Committee: \\ Richard LeSar, Major professor \\ Alan M. Russell \\ Scott Beckman \\ Ashraf Bastawros \\ Wei Hong \\ Iowa State University \\ Ames, Iowa \\ 2010 \\ Copyright (C) Caizhi Zhou, 2010. All rights reserved.
}




\section{TABLE OF CONTENTS}

LIST OF FIGURES ….......................................................................................

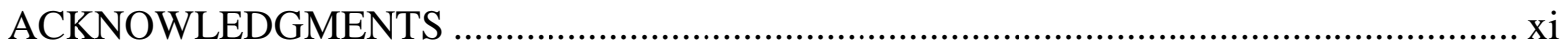

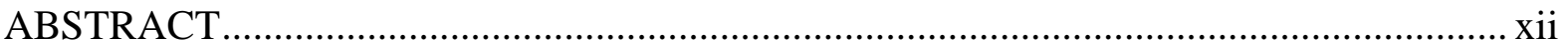

CHAPTER 1

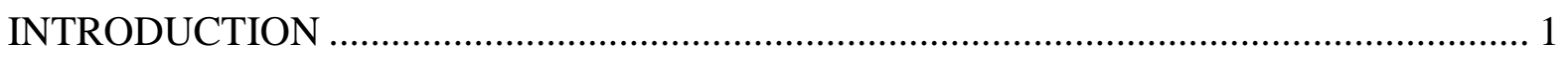

1.1 EXPERIMENTAL OBSERVATIONS OF PLASTICITY IN SINGLE CRYSTAL ...................................... 1

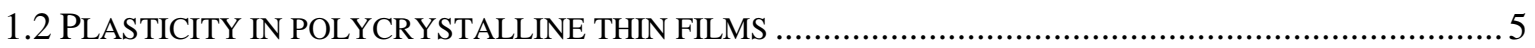

1.3 DISLOCATION DYNAMICS SIMULATIONS OF PLASTICITY IN SINGLE CRYSTALS ........................... 6

1.4 DISLOCATION DYNAMICS SIMULATIONS OF PLASTICITY IN THIN FILMS .................................. 9

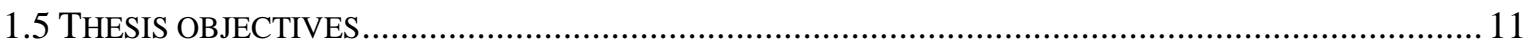

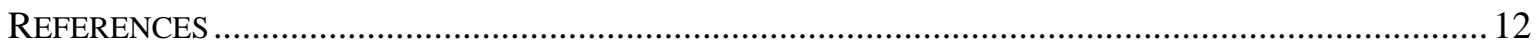

\section{CHAPTER 2}

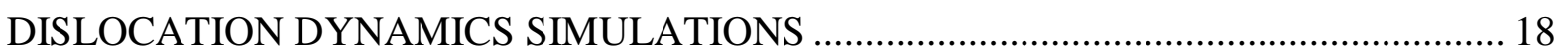

2.1 THE DISPLACEMENT FIELD OF DISLOCATIONS IN ISOTROPIC CRYSTALS .................................. 18

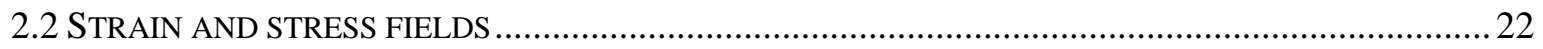

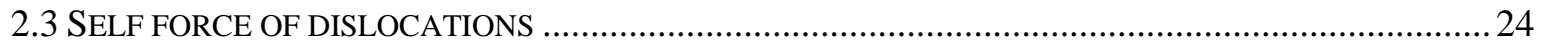

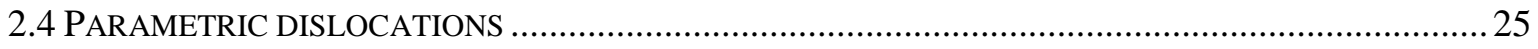

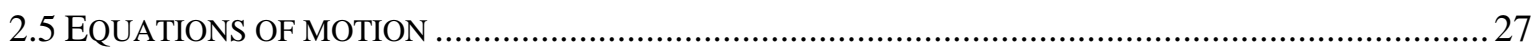

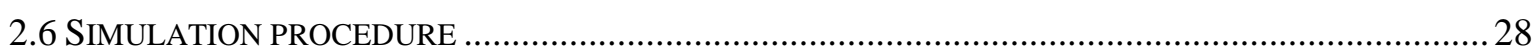

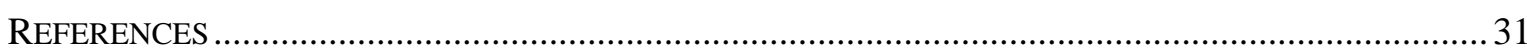

\section{CHAPTER 3}

IMAGE STRESSES IN DISLOCATION DYNAMICS SIMULATIONS ......................... 33

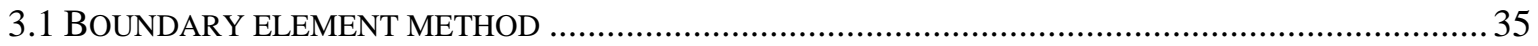

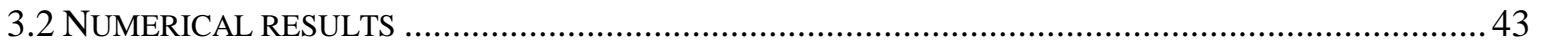

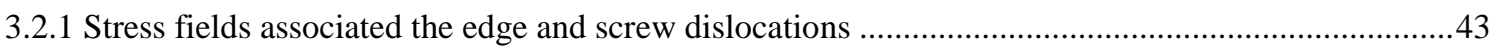

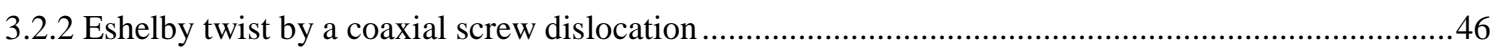

3.2.3 Image stress of a straight edge dislocation in a cylinder ................................................................4

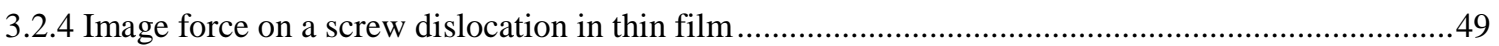


3.2.5 Effect of image stresses on the flow stress .50

REFERENCES

\section{CHAPTER 4}

SIZE EFFECTS ON PLASTICITY OF FCC SINGLE CRYSTALS .................................... 55

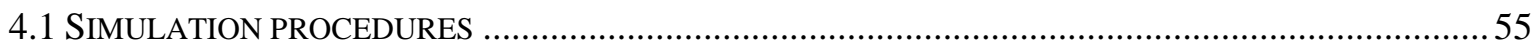

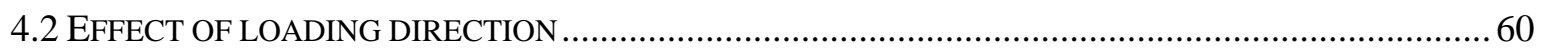

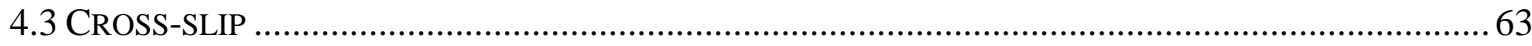

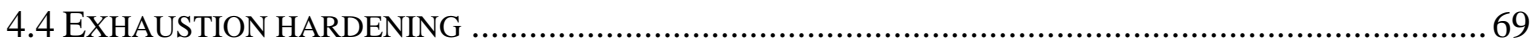

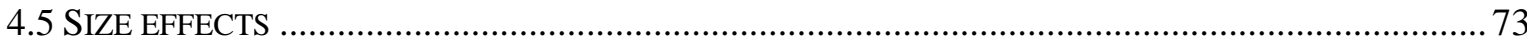

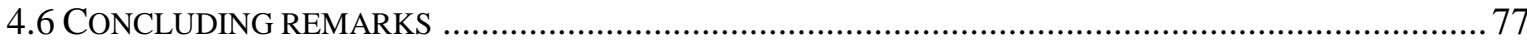

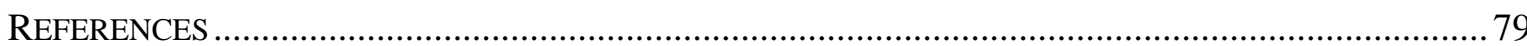

\section{CHAPTER 5}

PLASTIC DEFORMATION MECHANISMS OF FCC SINGLE CRYSTALS AT SMALL SCALES

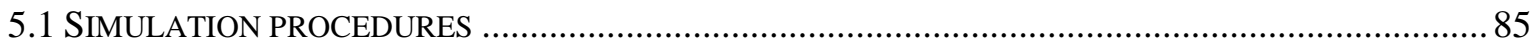

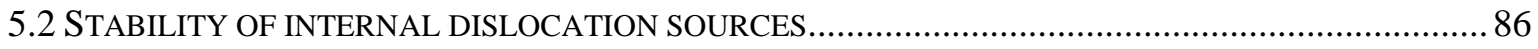

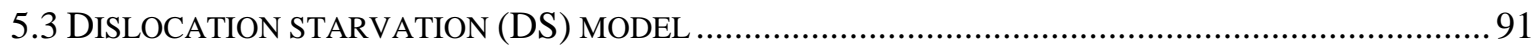

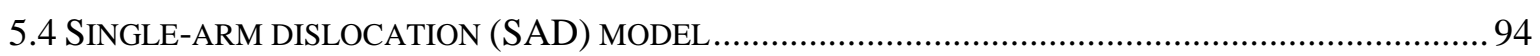

5.5 DISLOCATION INTERACTIONS CAUSING HARDENING AT SMALL SCALES...................................97

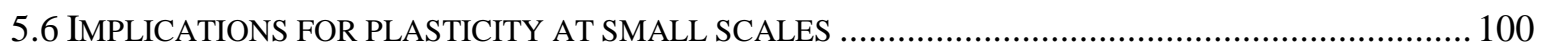

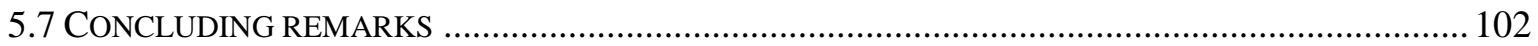

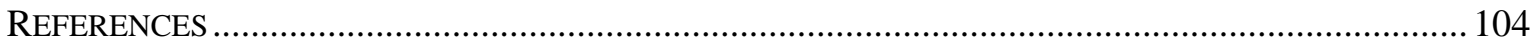

\section{CHAPTER 6}

SIMULATIONS OF THE EFFECT OF SURFACE COATINGS ON PLASTICITY AT

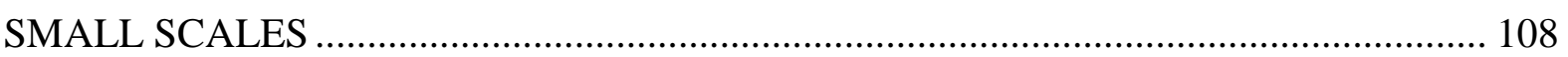

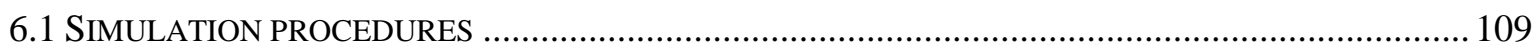

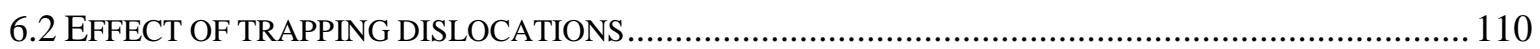

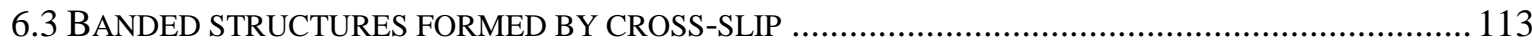

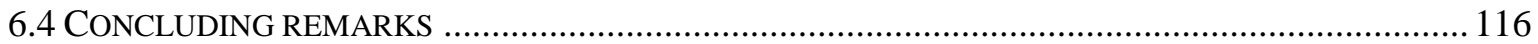

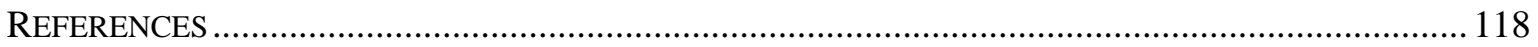

\section{CHAPTER 7}




\section{DISLOCATION DYNAMICS SIMULATIONS OF PLASTICITY IN}

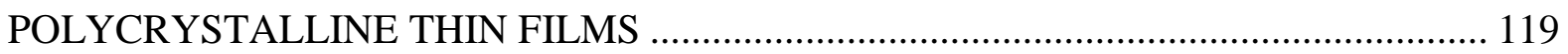

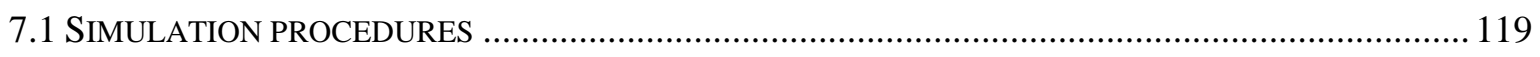

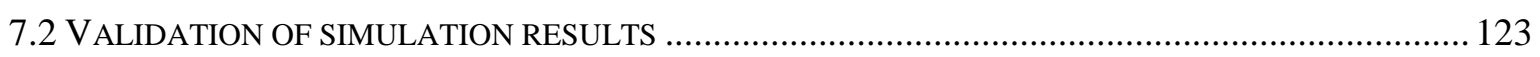

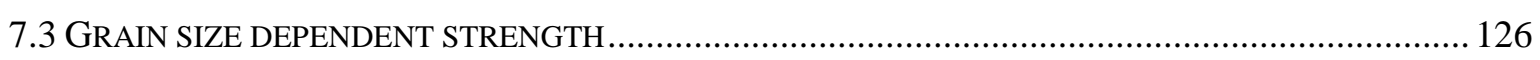

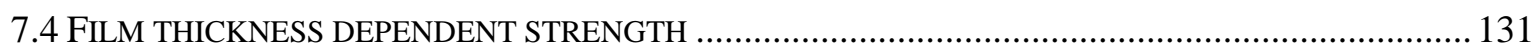

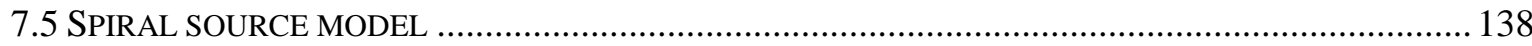

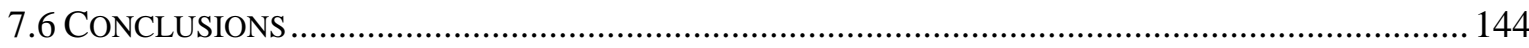

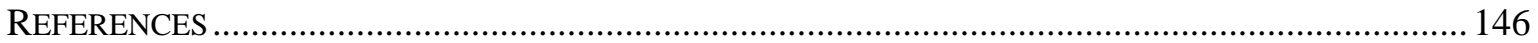

\section{CHAPTER 8}

DISLOCATION DYNAMICS SIMULATIONS OF BAUSCHINGER EFFECTS IN

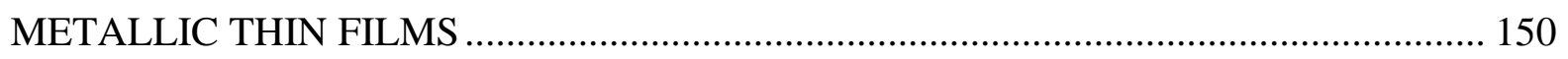

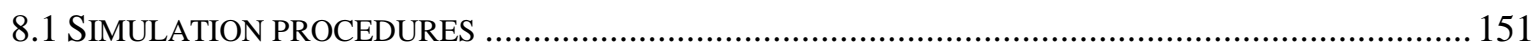

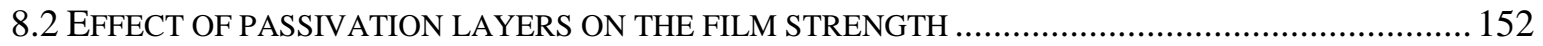

8.3 EFFECT OF PASSIVATION LAYERS ON REVERSE PLASTICITY OF THIN FILMS ............................... 154

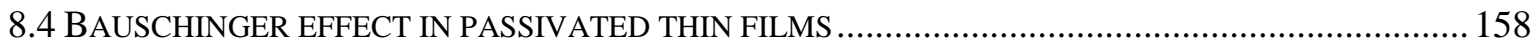

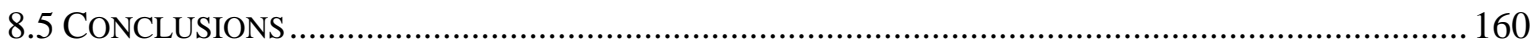

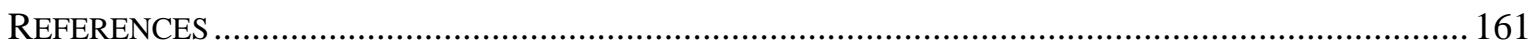

CHAPTER 9

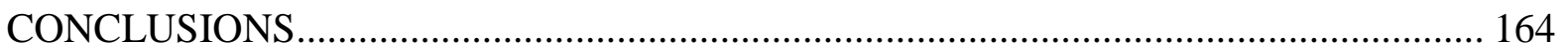




\section{LIST OF FIGURES}

Figure 1.1 (a)Schematic of the microcompression test, (b) Schematic of the flow response of a microcrystal oriented for single slip, (c) Scanning electron microscope (SEM) image of a $5 \mu \mathrm{m}$-diameter microcrystal sample of pure Ni oriented for single slip,

(d) SEM image of panel c after testing. 2

Figure 1.2 (a) stress-strain curves for Au micropillars under compression test, (b) stress-strain curves for Ni micropillars under compression test.

Figure 1.3 In situ TEM compression tests on a FIB microfabricated 160-nm-top-diameter Ni pillar with <111> orientation: (a) Dark-field TEM image of the pillar before the tests; note the high initial dislocation density, (b) Dark-field TEM image of the same pillar after the first test; the pillar is now free of dislocations. 5

Figure 2.1 Creation of a dislocation by a cut on the surface $(S)$

Figure 2.2 Representation of the solid angle, $\Omega$, at a field point $(Q)$ away from the dislocation loop line containing the set of points $(P)$.

Figure 2.3 Parametric representation of dislocation lines. (a) A dislocation loop is divided into segments connecting dislocation nodes; (b) a curved dislocation segment between two nodes

Figure 3.1 Stress and displacement fields associated an edge dislocation, (a) the configuration an edge dislocation created by inserting a half-plane of atoms, (b) 3D view of the stress and displacement field of an edge dislocation from numerical results, (c) the analytical solution of $\sigma \mathrm{xx}$ for an edge dislocation, and (d) the numerical results of $\sigma \mathrm{xx}$ for an edge dislocation (BEM mesh: 1734 elements, displacement magnification: 500, stress unit: $\mathrm{MPa})$.

Figure 3.2 Stress and displacement fields associated a screw dislocation, (a) the configuration a screw dislocation created by a "cut-and-slip" procedure in which the slip vector is parallel to the dislocation line, (b) 3D view of the stress and displacement field of a screw dislocation from numerical results, (c) the analytical solution of $\sigma x x$ for an edge dislocation, and (d) the numerical results of $\sigma \mathrm{xx}$ for an edge dislocation (BEM mesh: 1734 elements, displacement magnification: 500, stress unit: MPa) 
Figure 3.3 Numerical results of Eshelby twist by a coaxial screw dislocation, (a) the configuration of a coaxial screw dislocation in a meshed cylinder, (b) the distributions of displacement and stress fields from numerical results, (c) relative error in the twist between two cross-sections of a cylinder, located at $5 \mathrm{R}$ and $6 \mathrm{R}$ from the bottom surface, respectively, for different numbers of surface elements (Displacement magnification: 100)

Figure 3.4 The relative error as a function of the number of surface elements on the cylinder for the image force on an edge dislocation located at $d=0.3 r, 0.6 r$ and $0.9 r$. 48

Figure 3.5 Numerical results of image force on long screw dislocation in thin film, (a) the configuration of a screw dislocation in a meshed film, (b) comparison of numerical results with analytic results. .50

Figure 3.6 Comparison of flow stresses of the micropillars with and without image stresses .51

Figure 4.1 Dislocation structures in $3 \times 3 \times 3 \mu \mathrm{m}^{3}$ cube sample. (a) Initial dislocation structure in [111] view, (b) deformed structure in [001] view, (c) deformed structure in [110] view.

Figure 4.2 Dislocation structures in cut samples with $D=1.0 \mu \mathrm{m}$ (Dotted lines are BEM meshes). (a) Cutting from [001] before relaxation with $\rho=2.7 \times 10^{13} \mathrm{~m}^{-2}([111]$ view), (b) cutting from [001] after relaxation with $\rho=1.9 \times 10^{13} \mathrm{~m}^{-2}$ ([111] view), (c) cutting from [001] direction with $\rho=1.9 \times 10^{13} \mathrm{~m}^{-2}$ (upper [001] view), (lower [110] view), (d) cutting from [269] direction with $\rho=2.0 \times 10^{13} \mathrm{~m}^{-2}$ (upper [001] view), (lower [110] view).

Figure 4.3 Comparison of stress-strain curves of simulation and experiment. (a) Stress-strain and typical density-strain curves obtained from simulation with $D=1.0 \mu \mathrm{m}$, (b) Stress-strain curves obtained from experiment

Figure 4.4 Comparison of the stress and density evolution with and without cross-slip. (a) stress and density curves, (b) initial dislocation structure, (c) dislocation structure without cross-slip at $1 \%$ strain and (d) dislocation structure with cross-slip at $1 \%$ strain.

Figure 4.5 Plot of cross-slip on parallel dislocations and formation of prismatic loop (PL): (a) two parallel dislocations slip on its own planes, (b) one dislocation cross-slip under the attractive force, (c) collinear reaction of the leading segments forming two superjogs, (d) prismatic loops. 


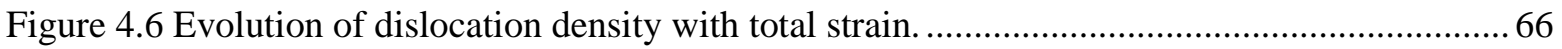

Figure 4.7 Plot of cross-slip forming dynamic FR source, see details in text.................................. 68

Figure 4.8 Configuration of superjog and dynamic spiral source, see details in text .......................70

Figure 4.9 Dislocation reactions causing flow intermittence: (a-d) glissile junction, (e-h) collinear reaction

Figure 4.10 (a) Stress-strain curves obtained from simulation with different sizes, (b) comparison log-log plot of the shear stress at $1 \%$ total strain of simulation results and experimental results.

Figure 4.11 Comparison log-log plot of the statistic model and simulation and experimental results. 76

Figure 5.1 Dislocation Nucleating and escaping from the surface of micropillar without considering image stresses (viewing along the Z-direction).

Figure 5.2 Dislocation nucleating from the surface and forming internal pinning points by cross-slip (CS) under the influence of image stresses (viewing along the Z-direction). .88

Figure 5.3 (a) Stress-strain curves and corresponding density-strain curves, (b) evolution of the number of internal dislocation sources.

Figure 5.4 Schematic sketch of one dislocation loop in a finite cylindrical sample with the distance, $v d t$, from free surfaces.

Figure 5.5 Comparison log-log plot of the general SAD model and microcompression results on various FCC single crystals.

Figure 5.6 Stress-strain and density-strain curves obtained from simulations on the sample with $D=1.0 \mu \mathrm{m}$. .98

Figure 5.7 Plot of dislocation configurations before and after hardening caused by dislocation interactions.

Figure 5.8 Complex deformation mechanism map for FCC single crystals: zone (I) nucleation of surface dislocations + starvation hardening, zone (II) nucleation/multiplication, depended on dislocation densities and structures, zone (III) multiplication of internal dislocations + exhaustion hardening. 101

Figure 6.1 Stress-strain curves for both coated and uncoated samples 110 
Figure 6.2 (a) Stress-strain and dislocation density-strain curves with diameter $D=1.0 \mu \mathrm{m}$, (b) initial dislocation structure, (c) dislocation structure in free-surface sample at $0.6 \%$ strain and (d) dislocation structure in coated sample at $0.6 \%$ strain.

Figure 6.3 (a) Stress-strain and dislocation density-strain curves with diameter $D=1.0 \mu \mathrm{m}$, (b) initial dislocation structure, (c) dislocation structure without cross-slip at $0.6 \%$ strain and $(\mathrm{d})$ dislocation structure with cross-slip at $0.6 \%$ strain.

Figure 6.4 Plot of double cross-slip in coated sample, see details in text. 116

Figure 7.1 Plot of the nine grain aggregate in DD simulations (Dashed lines are BEM mesh and dislocations are in color)

Figure 7.2 Illustration of a dislocation transmitting the tilt grain boundary according to the LT model: the incoming dislocation in the Grainl with Burgers vector, $b_{1}$, gradually bows out under the applied shear stress and then deposits a line segment along the GB; when the resolved shear stress at the GB dislocation exceeds the GB transmission strength, transmission occurs by punching a part of this deposited dislocation line onto Grain2 with Burgers vector, $b_{2}$, and left a residual dislocation with Burgers vector, $\Delta b=b_{2}-b_{1}$, in the GB plane to ensure conservation of the Burgers vector 122

Figure 7.3 Comparison of simulations results with experiment results: (a) stress-strain curves of simulation and experimental results on polycrystalline thin films with $500 \mathrm{~nm}$ grain size and $600 \mathrm{~nm}$ thickness; (b) evolution of dislocation densities; (c) dislocation structures in impenetrable GB case, (d) dislocation structures in free GB case; (e) dislocation structures in penetrable GB case with markers on transmitting GB dislocation sources. (Viewing along the [001]-direction).

Figure 7.4 Stress-strain plots comparing grain sizes at 250, 500, 1000 and $1500 \mathrm{~nm}$ for film thicknesses of (a) $250 \mathrm{~nm}$; (b) $500 \mathrm{~nm}$; (c) $1000 \mathrm{~nm}$ and (d) $1500 \mathrm{~nm}$..

Figure 7.5 Plots of total dislocation density vs. total strain in films with thicknesses of (a) 250 $\mathrm{nm}$; (b) $500 \mathrm{~nm}$; (c) $1000 \mathrm{~nm}$ and (d) $1500 \mathrm{~nm}$.

Figure 7.6 Plots of GB dislocation density vs. total strain in films with thicknesses of (a) 250 $\mathrm{nm}$; (b) $500 \mathrm{~nm}$; (c) $1000 \mathrm{~nm}$ and (d) $1500 \mathrm{~nm}$. 128

Figure 7.7 (a) Plot of yield stress vs. grain size, D, for the four film thicknesses. Solid line connecting the data points taken from samples with aspect ratio equal to one, above and below which data are taken from samples with low aspect ratio $(<1.0)$ and high 
aspect ratio (>1.0), respectively; (b) dislocation structures in the film with low aspect ratio, $(\mathrm{D}=1000 \mathrm{~nm}, \mathrm{H}=250 \mathrm{~nm}$ and $\mathrm{H} / \mathrm{D}=0.25)$ and (c) dislocation structures in the film with high aspect ratio, $(\mathrm{D}=250 \mathrm{~nm}, \mathrm{H}=1000 \mathrm{~nm}$ and $\mathrm{H} / \mathrm{D}=$ $4.0)$.

Figure 7.8 Stress-strain plots comparing different film thicknesses for grain sizes of (a) 250 $\mathrm{nm}$; (b) $500 \mathrm{~nm}$; (c) $1000 \mathrm{~nm}$ and (d) $1500 \mathrm{~nm}$.

Figure 7.9 Plots of total dislocation density vs. total strain in films with grain sizes of (a) 250 $\mathrm{nm}$; (b) $500 \mathrm{~nm}$; (c) $1000 \mathrm{~nm}$ and (d) $1500 \mathrm{~nm}$.

Figure 7.10 Plots of GB dislocation density vs. total strain in films with grain sizes of (a) 250 $\mathrm{nm}$; (b) $500 \mathrm{~nm}$; (c) $1000 \mathrm{~nm}$ and (d) $1500 \mathrm{~nm}$.

Figure 7.11 Dislocation structures in films with grain size equal $500 \mathrm{~nm}$ under $0.5 \%$ strain in different film thicknesses: (a) thicknesses equal $250 \mathrm{~nm}(\mathrm{H} / \mathrm{D}=0.5)$, upper in [001] view, lower in [ $\left[\begin{array}{lll}1 & 1 & 1\end{array}\right]$ view; (b) thicknesses equal $500 \mathrm{~nm}(\mathrm{H} / \mathrm{D}=1.0)$, upper in [001] view, lower in [ $\left[\begin{array}{lll}1 & 1 & 1\end{array}\right]$ view; (c) thicknesses equal $2000 \mathrm{~nm}(\mathrm{H} / \mathrm{D}=4.0)$, upper in [001] view, lower in [lllll 111 view. 135

Figure 7.12 Plots of mobile dislocation cross-slip when approaching the grain boundary dislocation: source $L 1$ and $L 2$ with $1 / 2[101](\overline{1} \overline{1} 1)$, source $L 3$ with $1 / 2[101](\overline{1} 11)$, and black lines indicating the grain boundaries, see details in text....... 136

Figure 7.13 Comparison of yield stresses from simulation and experiment results. Solid line connecting the data points taken from samples with aspect ratio equal to one, above and below which data are taken from samples with high aspect ratio $(>1.0)$ and low aspect ratio $(<1.0)$, respectively.

Figure 7.14 Schematic depiction of the operation of spiral source in freestanding thin films. Red lines are dislocations; $\mathrm{d} 1$ and $\mathrm{d} 2$ indicate the shortest distances of internal pinning point to the free surface and grain boundary; the spiral source operates in counterclockwise direction.

Figure 7.15 Schematic sketch of the statistical model for evaluating the effective length of spiral source in an equiaxed grain. Dashed lines indicate the axis of symmetry in the square.

Figure 7.16 Comparison of the results predicted by spiral source model stress with experimental data. The stress is shown versus the reciprocal value of the smaller dimension among film thickness or grain size. 143 
Figure 8.1 (a) Stress-strain curves of freestanding and passivated films under forward loading (dashed and solid lines for freestanding and passivated films, respectively); (b) dislocation structures in the $250 \mathrm{~nm}$ freestanding film; (c) dislocation structures in the $250 \mathrm{~nm}$ passivated film.

Figure 8.2 (a) Stress-strain curves of freestanding and passivated films during unloading (H and $\mathrm{D}$ are both equal to $500 \mathrm{~nm}$ ); (b) the corresponding total dislocation density evolution in both cases; (c) the corresponding grain boundary dislocation density evolution and interface dislocation density evolution in the passivated film.

Figure 8.3 Illustration of the reversed motion of the pile-up dislocation (marked with arrow) in passivated films during unloading.

Figure 8.4 (a) Description of notations used for quantifying BE, $\varepsilon_{y}$ denotes yield strain, $\varepsilon_{\text {pre }}$ denotes pre- strain and $\varepsilon_{B E}$ denotes BE strain; (b) plot of normalized BE strain vs normalized pre-strain from simulation results on passivated films with different aspect ratios and comparison with experiment results 159 


\section{ACKNOWLEDGMENTS}

I wish to express my sincere appreciation to my major professor, Dr. Richard LeSar for his understanding, guidance and encouragement throughout my $\mathrm{PhD}$ study. I would also like to thank the other committee members, Prof. Alan M. Russell, Prof. Scott Beckman, Prof. Ashraf Bastawros and Prof. Wei Hong for providing insightful suggestions and taking time to serve on my committee.

Gratitude should also been given to Ames Laboratory operated for the U.S. Department of Energy by Iowa State University, supporting this project.

Thanks also go to Dr. Bulent Biner in Ames Laboratory, Dr. Zhiqiang Wang in University of North Texas and Dr. Dennis Dimiduk in Air Force Research Laboratory for their help and interesting discussions. 


\begin{abstract}
As metallic structures and devices are being created on a dimension comparable to the length scales of the underlying dislocation microstructures, the mechanical properties of them change drastically. Since such small structures are increasingly common in modern technologies, there is an emergent need to understand the critical roles of elasticity, plasticity, and fracture in small structures. Dislocation dynamics (DD) simulations, in which the dislocations are the simulated entities, offer a way to extend length scales beyond those of atomistic simulations and the results from DD simulations can be directly compared with the micromechanical tests.
\end{abstract}

The primary objective of this research is to use 3-D DD simulations to study the plastic deformation of nano- and micro-scale materials and understand the correlation between dislocation motion, interactions and the mechanical response. Specifically, to identify what critical events (i.e., dislocation multiplication, cross-slip, storage, nucleation, junction and dipole formation, pinning etc.) determine the deformation response and how these change from bulk behavior as the system decreases in size and correlate and improve our current knowledge of bulk plasticity with the knowledge gained from the direct observations of small-scale plasticity. Our simulation results on single crystal micropillars and polycrystalline thin films can march the experiment results well and capture the essential features in small-scale plasticity. Furthermore, several simple and accurate models have been developed following our simulation results and can reasonably predict the plastic behavior of small scale materials. 


\section{CHAPTER 1}

\section{INTRODUCTION}

The mechanical properties of materials change drastically when specimen dimensions are smaller than a few micrometers. Since such small structures are increasingly common in modern technologies, there is an emergent need to understand the critical roles of elasticity, plasticity, and fracture in small structures. Small-scale structures also offer opportunities for direct comparison between modeling and experiment at previously inaccessible scales. The experiments provide data for validation of models, and the models provide a path for new, physically-based understanding and prediction of materials behavior. Mechanical tests at nanometer or micrometer scales are difficult to perform, but they provide guidance to develop new technologies and new theories of plasticity. Experimental studies on the mechanical behavior of small structures are not new; the first work on thin metal whiskers (with diameters of $\sim 100$ microns) occurred more than 50 years ago [1]. The past few years, however, have seen a major leap forward in the experimental study of small samples. We focus here on studies of metals, highlighting examples of previous work.

\subsection{Experimental observations of plasticity in single crystal}

Uchic et al. recently pioneered the study of size effects in compression of 1-micron diameter metal samples as shown in Figure 1.1 [2-6]. Cylindrical pillars with varying radii were machined with a focused-ion beam (FIB) from single-crystal bulk samples and compressed by a blunted nanoindentor. This pioneering work spurred similar activities from several groups, with studies on sub-micron to many-micron sample sizes [7-14]. Studies on face-centered cubic (fcc) metals show that flow stress increases as system size decreases, with the onset of deviation from bulk behavior varying somewhat from material to material. 

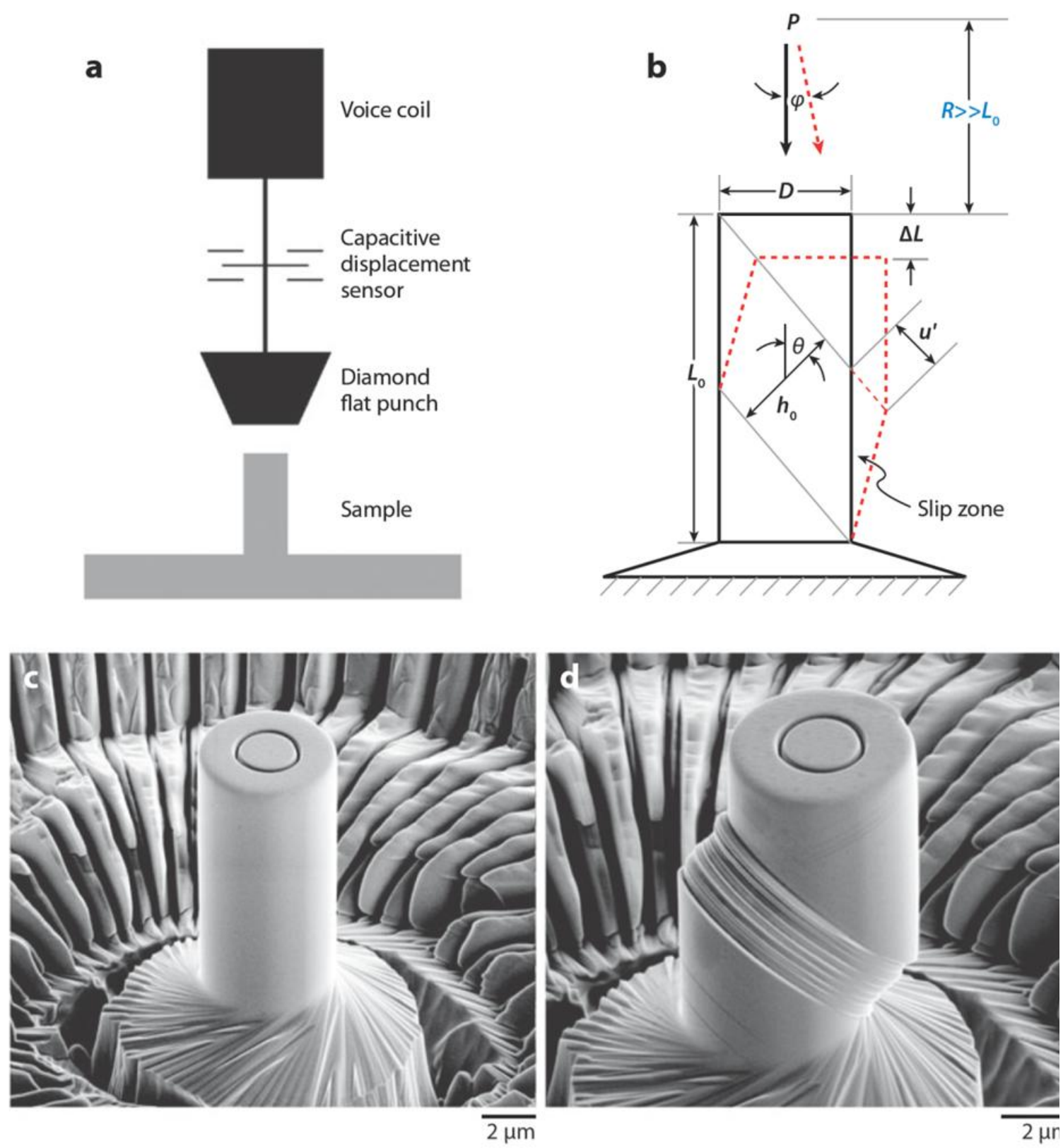

Figure 1.1 (a)Schematic of the microcompression test, (b) Schematic of the flow response of a microcrystal oriented for single slip, (c) Scanning electron microscope (SEM) image of a $5 \mu \mathrm{m}$-diameter microcrystal sample of pure Ni oriented for single slip, (d) SEM image of panel c after testing [2]. 
The increased flow stress is accompanied by extremely large strain hardening at small to moderate strains, with small samples showing higher strain-hardening rates in Figure 1.2. [2, $8,14]$. Indeed, very small samples can achieve extremely high flow stresses, e.g., a cylinder with a diameter of about 0.2 micron in nickel can sustain a stress of up to $2 \mathrm{GPa}$ [11]. This general result that yield stress increases as system size decreases is also found in other tests on fcc materials, including a study using an atomic force microscope (AFM) to bend gold nanowires [15] and also in polycrystalline membranes of copper, gold, and aluminum in pure tension [16]. Probably the most accepted explanation of these size effects is the "dislocation starvation" model [9-11], in which dislocations are drawn to free surfaces by strong image forces and exit the crystal. Recent work on body-centered cubic (bcc) molybdenum alloys showed that both the initial yield stress and size-dependent hardening rate are strongly dependent on initial dislocation density [17], an issue not well studied in the fcc metals.

Key to an understanding of these size effects is a characterization of the internal structure of microscale samples. Some work has been done with transmission electron microscopy (TEM), but there are limitations of the thickness of samples that can be studied with TEM - thin foils must be cut from the samples and the results thus depend on the plane of the foils as well as the size and orientation of the microstructures. Results from these studies are reasonably consistent, however, showing a small net increase in dislocation density after the initial loading $[10,12]$. A recent study using a novel in situ TEM micropillar method showed evidence of "mechanical annealing," a sudden drop in dislocation density upon initial loading and a subsequent small increase in density with further compression [18]. The dislocation structures before and after deformation are shown in Figure 1.3. Micro x-ray diffraction (XRD) studies [19-21] of lattice rotations in these systems indicate approximately the same dislocation contents as TEM measurements. Overall, it is clear that dislocation densities and activities are greatly affected by system size, but the connection between size-dependent strengthening and dislocation activity is not yet clearly established. 

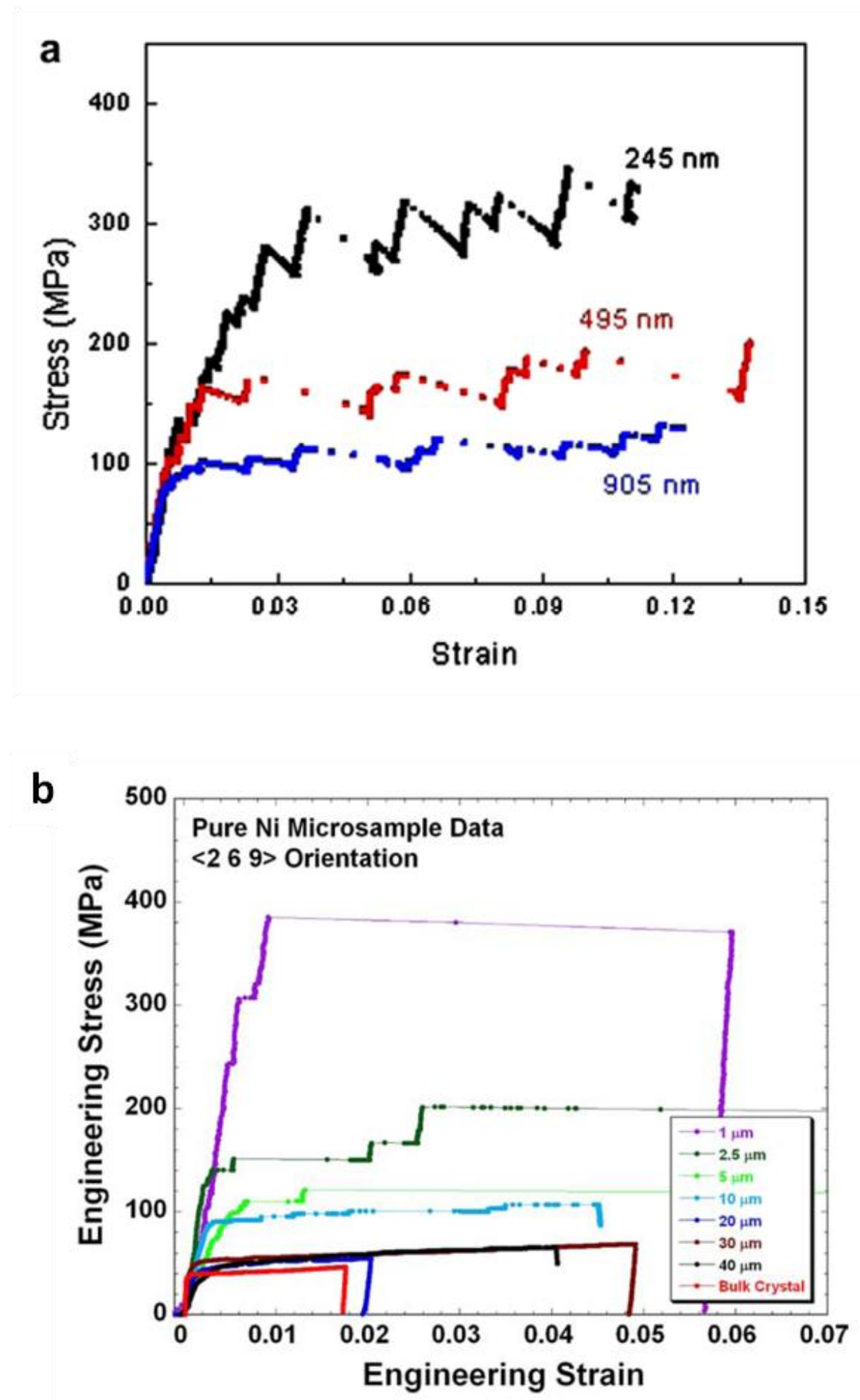

Figure 1.2 (a) stress-strain curves for $\mathrm{Au}$ micropillars under compression test [65], (b) stress-strain curves for Ni micropillars under compression test [2]. 

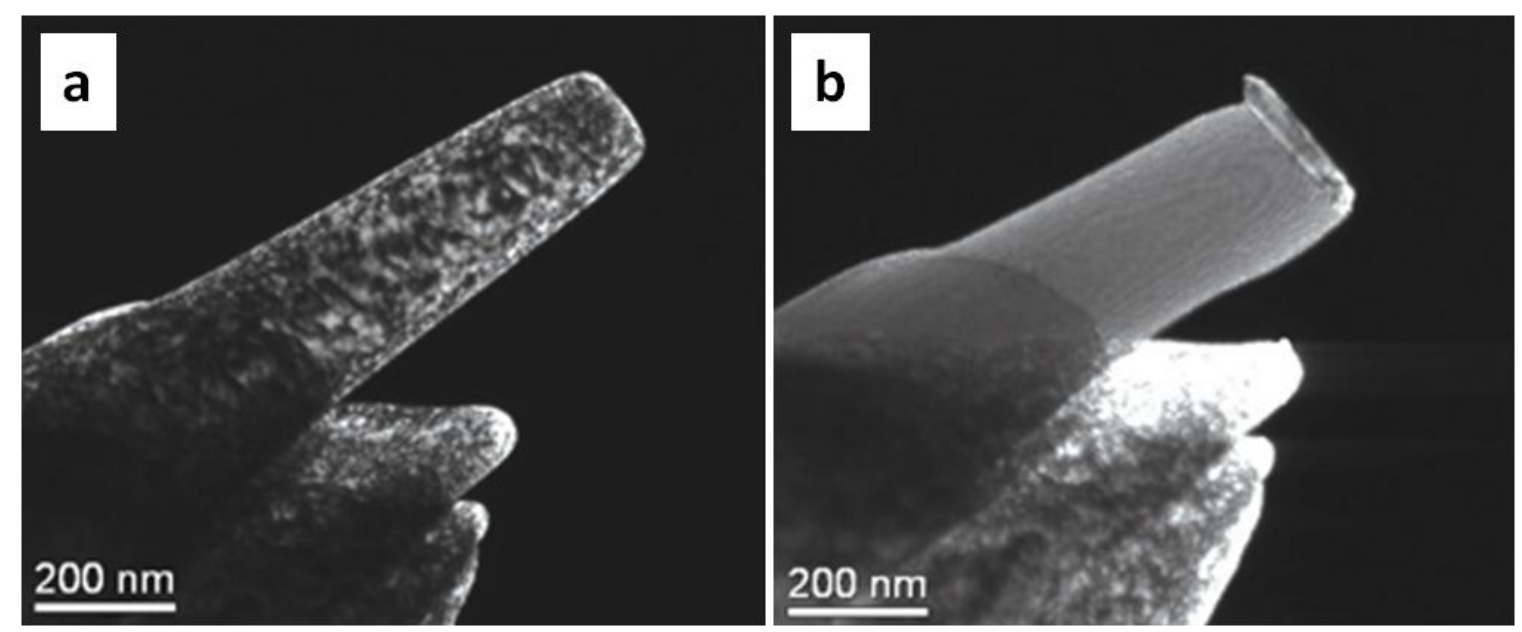

Figure 1.3 In situ TEM compression tests on a FIB microfabricated 160-nm-top-diameter Ni pillar with <111> orientation: (a) Dark-field TEM image of the pillar before the tests; note the high initial dislocation density, (b) Dark-field TEM image of the same pillar after the first test; the pillar is now free of dislocations [18].

\subsection{Plasticity in polycrystalline thin films}

One of the most important phenomena in metallic thin films is that their strength differs significantly from that of the corresponding bulk materials when their dimensions become comparable to the length scales of the underlying dislocation microstructures. Although this phenomenon has been known for quite a long time, a full understanding of thin film plasticity has neither experimentally nor theoretically been obtained [22-23].

In general, the yield stress of metallic thin films increases with decreasing the film thickness and/or grain size and the scaling behavior of the yield stress with varying film thickness or grain size is described in power-law form [24]. Experimental results for polycrystalline films reveal different scaling exponents ranging from -0.5 to -1 [25-27]. So far, two kind models are widely used to describe the observed size effect in thin films. The first one is Nix-Freund model [28-31] that considered dislocations channeling through the film are forced to deposit interfacial dislocation segments at the film/substrate interface, and 
explained the size dependent plasticity in single crystal thin films as a consequence of geometrical constraints on dislocations in thin films. This kind model can give an exponent of scaling behavior between film thickness and yield strength close to -1 . Another kind model for thin film plasticity is based on Hall-Petch-like behavior [32-33] that dislocations are assumed to totally pile-up at grain boundaries or the film/substrate interface and the effective sizes of dislocation sources will shrink due to a reduction in the effective grain size or film thickness by previously pile-up dislocations [34-35]. In contrast to Nix-Freund models, an exponent of -0.5 on the scaling was predicted by these models. Up to now, none of existed models seem to describe the plastic behavior of polycrystalline films in a satisfactory manner. Undoubtedly, dislocation interactions are important in determining the strengthening of thin films and can be more complicated than those considered in analytical calculations. Thus, a detailed understanding of dislocation motion, multiplication and interactions in a confined geometry is the key to explain the plastic deformation of polycrystalline thin films.

\subsection{Dislocation dynamics simulations of plasticity in single crystals}

The recent increase in experimental deformation data in confined geometries has been accompanied by a similar focus on use of modeling and simulation on small samples. Discrete dislocation simulations, in which the dislocations are the simulated entities, offer a way to extend length scales beyond those of atomistic simulations [36-40]. Simply put, dislocation-based simulations (1) represent the dislocation line in some convenient way, (2) determine either the forces or interaction energies between dislocations, and (3) calculate the structures and response of the dislocations to external stresses. These simulations are useful for mapping out the underlying mechanisms by providing "data" not available experimentally on, for example, dislocation ordering, evolution of large-scale dislocation structures (walls, cells, pile ups), dynamics (avalanches and instabilities), etc. For the 
micron-scale systems described above, recent DD simulations have provided important insights into the mechanisms that determine the size-affected mechanical response.

The first attempts to explain the micropillar results using DD simulations assumed two-dimensional (2-D) models. Deshpande and colleagues [41-43] examined the uniaxial deformation of 2-D simulation cells under constrained and unconstrained flow in which only one slip system was operative. In these studies, the mean and variance of the dislocation source strengths, obstacle spacing, and obstacle strength were selected to be independent of the simulation-cell size. For unconstrained simulations, the size dependency displayed in these 2-D DD simulations can be attributed to dislocation pinning, and subsequent pileups were more likely to occur in larger cells, which resulted in stronger local fluctuations of the stress field that lowered the applied stress needed to sustain plastic flow. Conversely, in the constrained simulations, almost no size dependency was observed with flow-softening behavior and all cells were able to establish internal dislocation-density gradients in order to satisfy the boundary conditions, thus locally augmenting the internal stress field and mobile segment population and mitigating the influences of cell size. After that, Benzerga and colleagues [44-45] also developed 2-D DD simulations including different rules for the effects from junction formation and source or obstacle creation. In contrast to the investigations by Deshpande et al., Benzerga and colleagues randomly assigned each dislocation source a length whereby the maximum possible length was dependent upon the cell size. These 2-D DD simulations displayed a size-dependent increase in the proportional limit with decreasing simulation-cell size, which was attributed to the change in the source activation stress for the few largest sources in any given cell. That is, the simulated material strength was directly related to the weakest source. However, whereas most simulated stress-strain curves displayed little-to-no strain hardening after initial yield, in smaller cells, dislocation pinning and subsequent blocking of sources produced strain hardening rates that approached the elastic limit. Although 2-D DD SIMULATIONS studies demonstrated a 
size-affected flow stress or strain hardening rate, the overall simulation-cell response is unlike most experimental data, especially with regard to the change in strain-hardening behavior at initial yield.

Recent 3-D DD simulations developed by a number of groups employing a variety of approximations and models have some significant advantages over the aforementioned 2-D DD SIMULATIONS ; for example, the local interactions between dislocations can be naturally accounted for, and the motion of dislocations, especially those that interact with the free surfaces of the microcrystal, can be more accurately modeled [5, 46-52]. In initial studies, the set of isolated Frank-Read sources (FRs) with rigidly fixed ends was widely employed as the starting dislocation populations [5, 46, 48-50, 52]. Tang et al., using a fixed number of Frank-Read (FR) sources as the initial condition, stated that dislocation escape through free surfaces plays a significant role in the size dependence of the plastic response of single-crystals [50]. Rao et al. found that the intermittency of plastic flow in small samples was normally caused by forest interactions [48]. Senger et al. argued that the observed size effect is not pronounced in samples larger than $2 \mu \mathrm{m}$ and the flow stress in small pillars is affected more strongly by dislocation reactions than in larger samples [49]. Meanwhile, El-Awady et al. demostrated the effect of the weakest dislocation sources in samples and cross-slip lead to additional strengthening and discontinuous on the stress-strain curves [5]. In addition, Parthasarathy et al. developed a statistical model for the flow strength of small samples, which was entirely based on the stochastics of spiral source (single-arm source) lengths in samples of finite size [53]. However, real dislocation structures in experiments are much more complicated than the set of isolated FR sources used as the initial configuration in most previous DD simulations. The recent study by Tang et al. [51] differed that the initial source distribution is not FRs predefined; rather, used artificially generated jogged dislocations as starting dislocation populations for their simulations while neglected the boundary conditions and cross-slip, and showed that sources shut-down causes staircase 
behavior observed in experiments. Motz et al. [47] used the dislocation structures relaxed from high dense dislocation loops as the initial input for DD simulations, and reported the flow stress at $0.2 \%$ plastic deformation scaled with specimen size with an exponent between -0.6 and -0.9 , depending on the initial structure and size regime. That is still under debates since most pillars have been made from well-annealed single crystals or sputtered thin films which do not involve such high densities of dislocation interactions [54]. Despite these progresses, there are still many unanswered questions regarding the plasticity at small scales, such as whether cross-slip is possible, how the image stresses induced by free surface and confined geometries influence multiplication of dislocation sources and the effect of crystal orientation (multi-slip versus single slip).

\subsection{Dislocation dynamics simulations of plasticity in thin films}

Initial attempts to explain the thin film plasticity using DD assumed two-dimensional (2-D) models. Nicola and coworkers conducted a serial of 2-D simulations on polycrystalline thin films and concluded that the yield strength of freestanding thin films is nearly independent of film thickness and the size effect results from the dislocation pile-ups at impenetrable interfaces, such as grain boundaries and passivation layers [35, 55-57]. Hartmaier et al. modeled polycrystalline films by incorporating dislocation climb in their 2-D simulations and showed the dislocation slip mechanism will be dominant in thicker films, while the creep mechanism prevails in ultra-thin films with thickness below $400 \mathrm{~nm}$ [58]. Han et al. investigated the surface induced size effects through 2-D simulations and the results indicated that a free surface might act either as a dislocation sink or as a net dislocation source that induced harder as well as softer deformation behaviors in a crystalline solid [59]. However, 2-D simulations cannot capture real microstructures in materials and are unlikely to describe thin film phenomena accurately, because dislocations are treated as infinitely long and parallel to each other, and also dislocation interactions are almost 
neglected in 2-D DD, which are important in the plastic deformation of a real specimen. Fortunately, full 3-D simulations can be used to understand these features of thin film mechanical behavior. In 3-D DD simulations, every dislocation configuration is decomposed into a succession of elementary segments, which can move under the external forces in discrete steps and generate more realistic dislocation structures. Pant et al. [60] employed 3-D DD simulations to study the interaction of threading dislocations in face-centered cubic (FCC) metal films. They found that different dislocation interactions dominate film behavior in different ranges of film thickness and applied strain, thus simple analytical calculations are unlikely to describe film phenomena. von Blanckenhagen et al. [61] investigated the plastic deformation of polycrystalline FCC metal thin films by simulating the dynamics of discrete dislocations in a representative columnar grain. Their simulations showed an inverse dependence of the flow stress on film thickness and the dependence of the hardening rate on film thickness can be reproduced by using an initial dislocation source density independent of grain dimensions. Espinosa et al. [62-63] assumed all dislocation sources were located at grain boundaries in their 3-D DD simulations and proposed a new interpretation of size scale plasticity of thin films in their study based on the probability of activating grain boundary dislocation sources. Recently, Fertig and Baker [64] conducted 3-D DD simulations on single crystal thin films and demonstrated that weak dislocation interactions still survive at high stress level, due to the inhomogeneity of the stress field in the film, and the mean free path for dislocation motion is closely related to the inhomogeneous stress distribution. So far, none of previous DD simulations on thin films considered stress relaxation mechanisms in their models, such as cross-slip of dislocations and dislocations transmitting at grain boundaries, and thus it is still unclear how these dynamic behaviors of dislocations will affect mechanical properties of polycrystalline thin films. In order to generate simple, accurate models that can be used to predict film behavior, there is an emergent need to identify the critical features in the plastic deformation of polycrystalline thin films, which can be accomplished by full 3-D DD simulations including basic dislocation mechanisms. 


\subsection{Thesis objectives}

The primary objective of this thesis is to incorporate boundary-element method (BEM) into 3-D DD SIMULATIONS to calculate the surface forces and incorporate the thermally-activated cross-slip to study the plastic deformation of nano- and micro-scale materials and understand the correlation between dislocation motion and the mechanical response. Specifically, to identify what critical events (i.e., dislocation multiplication, cross-slip, storage, nucleation, junction and dipole formation, pinning etc.) determine the deformation response and how these change from bulk behavior as the system decreases in size and correlate and improve our current knowledge of bulk plasticity with the knowledge gained from the direct observations of small-scale plasticity. 


\section{References}

[1] Herring C, Galt JK. Elastic and plastic properties of very small metal specimens. Physical Review 1952;85:1060.

[2] Dimiduk DM, Uchic MD, Parthasarathy TA. Size-affected single-slip behavior of pure nickel microcrystals. Acta Materialia 2005;53:4065.

[3] Dimiduk DM, Uchic MD, Rao SI, Woodward C, Parthasarathy TA. Overview of experiments on microcrystal plasticity in FCC-derivative materials: selected challenges for modelling and simulation of plasticity. Modelling and Simulation in Materials Science and Engineering 2007;15:135.

[4] Dimiduk DM, Woodward C, LeSar R, Uchic MD. Scale-free intermittent flow in crystal plasticity. Science 2006;312:1188.

[5] El-Awady JA, Wen M, Ghoniem NM. The role of the weakest-link mechanism in controlling the plasticity of micropillars. Journal of the Mechanics and Physics of Solids 2009;57:32.

[6] Uchic MD, Dimiduk DM, Florando JN, Nix WD. Sample dimensions influence strength and crystal plasticity. Science 2004;305:986.

[7] Brinckmann S, Kim JY, Greer JR. Fundamental differences in mechanical behavior between two types of crystals at the nanoscale. Physical Review Letters 2008;100.

[8] Frick CP, Clark BG, Orso S, Schneider AS, Arzt E. Size effect on strength and strain hardening of small-scale 111 nickel compression pillars. Materials Science and Engineering a-Structural Materials Properties Microstructure and Processing 2008;489:319.

[9] Greer JR, Nix WD. Size dependence of mechanical properties of gold at the sub-micron scale. Applied Physics a-Materials Science \& Processing 2005;80:1625.

[10] Greer JR, Nix WD. Nanoscale gold pillars strengthened through dislocation starvation. Physical Review B 2006;73.

[11] Greer JR, Oliver WC, Nix WD. Size dependence of mechanical properties of gold at the micron scale in the absence of strain gradients. Acta Materialia 2005;53:1821.

[12] Ng KS, Ngan AHW. Stochastic nature of plasticity of aluminum micro-pillars. Acta Materialia 2008;56:1712. 
[13] Ng KS, Ngan AHW. Stochastic theory for jerky deformation in small crystal volumes with pre-existing dislocations. Philosophical Magazine 2008;88:677.

[14] Volkert CA, Lilleodden ET. Size effects in the deformation of sub-micron Au columns. Philosophical Magazine 2006;86:5567.

[15] Wu B, Heidelberg A, Boland JJ. Mechanical properties of ultrahigh-strength gold nanowires. Nature Materials 2005;4:525.

[16] Espinosa HD, Prorok BC, Peng B. Plasticity size effects in free-standing submicron polycrystalline FCC films subjected to pure tension. Journal of the Mechanics and Physics of Solids 2004;52:667.

[17] Bei H, Shim S, Pharr GM, George EP. Effects of pre-strain on the compressive stress-strain response of Mo-alloy single-crystal micropillars. Acta Materialia 2008;56:4762.

[18] Shan ZW, Mishra RK, Asif SAS, Warren OL, Minor AM. Mechanical annealing and source-limited deformation in submicrometre-diameter Ni crystals. Nature Materials 2008;7:115.

[19] Budiman AS, Han SM, Greer JR, Tamura N, Patel JR, Nix WD. A search for evidence of strain gradient hardening in Au submicron pillars under uniaxial compression using synchrotron X-ray microdiffraction. Acta Materialia 2008;56:602.

[20] Maass R, Grolimund D, Van Petegem S, Willimann M, Jensen M, Van Swygenhoven H, Lehnert T, Gijs MAM, Volkert CA, Lilleodden ET, Schwaiger R. Defect structure in micropillars using X-ray microdiffraction. Applied Physics Letters 2006;89.

[21] Maaß R, Van Petegem S, Zimmermann J, Borca CN, Van Swygenhoven H. On the initial microstructure of metallic micropillars. Scripta Materialia 2008;59:471.

[22] Kraft O, Volkert CA. Mechanical testing of thin films and small structures. Advanced Engineering Materials 2001;3:99.

[23] Nix WD. Mechanical-properties of thin-films. Metallurgical Transactions a-Physical Metallurgy and Materials Science 1989;20:2217.

[24] Kraft O, Freund LB, Phillips R, Arzt E. Dislocation plasticity in thin metal films. Mrs Bulletin 2002;27:30.

[25] Keller RM, Baker SP, Arzt E. Quantitative analysis of strengthening mechanisms in thin $\mathrm{Cu}$ films: Effects of film thickness, grain size, and passivation. Journal of Materials Research 1998;13:1307. 
[26] Venkatraman R, Bravman JC. Separation of film thickness and grain-boundary strengthening effects in al thin-films on si. Journal of Materials Research 1992;7:2040.

[27] Yu DYW, Spaepen F. The yield strength of thin copper films on Kapton. Journal of Applied Physics 2004;95:2991.

[28] Freund LB. The stability of a dislocation threading a strained layer on a substrate. Journal of Applied Mechanics-Transactions of the Asme 1987;54:553.

[29] Freund LB. The mechanics of dislocations in strained-layer semiconductor-materials. Advances in Applied Mechanics, Vol 30, vol. 30. San Diego: Academic Press Inc, 1994. p.1.

[30] Freund LB, Nix WD. Critical thickness condition for a strained compliant substrate/epitaxial film system. Applied Physics Letters 1996;69:173.

[31] Nix WD. Yielding and strain hardening of thin metal films on substrates. Scripta Materialia 1998;39:545.

[32] Hall EO. The deformation and ageing of mild steel .3. discussion of results. Proceedings of the Physical Society of London Section B 1951;64:747.

[33] Petch NJ. The cleavage strength of polycrystals. Journal of the Iron and Steel Institute $1953 ; 174: 25$.

[34] Friedman LH, Chrzan DC. Continuum analysis of dislocation pile-ups: influence of sources. Philosophical Magazine a-Physics of Condensed Matter Structure Defects and Mechanical Properties 1998;77:1185.

[35] Nicola L, Van der Giessen E, Needleman A. Discrete dislocation analysis of size effects in thin films. Journal of Applied Physics 2003;93:5920.

[36] Devincre B, Kubin L, Hoc T. Physical analyses of crystal plasticity by DD simulations. Scripta Materialia 2006;54:741.

[37] Ghoniem NM, Tong SH, Sun LZ. Parametric dislocation dynamics: A thermodynamics-based approach to investigations of mesoscopic plastic deformation. Physical Review B 2000;61:913.

[38] Gulluoglu AN, Srolovitz DJ, Lesar R, Lomdahl PS. Dislocation distributions in 2 dimensions. Scripta Metallurgica 1989;23:1347.

[39] Kubin LP, Canova G. The modeling of dislocation patterns. Scripta Metallurgica Et Materialia 1992;27:957. 
[40] Zbib HM, de la Rubia TD, Rhee M, Hirth JP. 3D dislocation dynamics: stress-strain behavior and hardening mechanisms in fcc and bcc metals. Journal of Nuclear Materials 2000;276:154.

[41] Balint DS, Deshpande VS, Needleman A, Van der Giessen E. Size effects in uniaxial deformation of single and polycrystals: a discrete dislocation plasticity analysis. Modelling and Simulation in Materials Science and Engineering 2006;14:409.

[42] Deshpande VS, Needleman A, Van der Giessen E. Plasticity size effects in tension and compression of single crystals. Journal of the Mechanics and Physics of Solids 2005;53:2661.

[43] Deshpande VS, Needleman A, Van der Giessen E. Discrete dislocation plasticity analysis of single slip tension. Materials Science and Engineering a-Structural Materials Properties Microstructure and Processing 2005;400:154.

[44] Benzerga AA, Shaver NF. Scale dependence of mechanical properties of single crystals under uniform deformation. Scripta Materialia 2006;54:1937.

[45] Guruprasad PJ, Benzerga AA. Size effects under homogeneous deformation of single crystals: A discrete dislocation analysis. Journal of the Mechanics and Physics of Solids 2008;56:132.

[46] El-Awady JA, Biner SB, Ghoniem NM. A self-consistent boundary element, parametric dislocation dynamics formulation of plastic flow in finite volumes. Journal of the Mechanics and Physics of Solids 2008;56:2019.

[47] Motz C, Weygand D, Senger J, Gumbsch P. Initial dislocation structures in 3-D discrete dislocation dynamics and their influence on microscale plasticity. Acta Materialia 2009;57:1744.

[48] Rao SI, Dimiduk DM, Parthasarathy TA, Uchic MD, Tang M, Woodward C. Athermal mechanisms of size-dependent crystal flow gleaned from three-dimensional discrete dislocation simulations. Acta Materialia 2008;56:3245.

[49] Senger J, Weygand D, Gumbsch P, Kraft O. Discrete dislocation simulations of the plasticity of micro-pillars under uniaxial loading. Scripta Materialia 2008;58:587.

[50] Tang H, Schwarz KW, Espinosa HD. Dislocation escape-related size effects in single-crystal micropillars under uniaxial compression. Acta Materialia 2007;55:1607.

[51] Tang H, Schwarz KW, Espinosa HD. Dislocation-source shutdown and the plastic behavior of single-crystal micropillars. Physical Review Letters 2008;100. 
[52] Weygand D, Poignant M, Gumbsch P, Kraft O. Three-dimensional dislocation dynamics simulation of the influence of sample size on the stress-strain behavior of fcc single-crystalline pillars. Materials Science and Engineering a-Structural Materials Properties Microstructure and Processing 2008;483:188.

[53] Parthasarathy TA, Rao SI, Dimiduk DM, Uchic MD, Trinkle DR. Contribution to size effect of yield strength from the stochastics of dislocation source lengths in finite samples. Scripta Materialia 2007;56:313.

[54] Lee SW, Han SM, Nix WD. Uniaxial compression of fcc Au nanopillars on an $\mathrm{MgO}$ substrate: The effects of prestraining and annealing. Acta Materialia 2009;57:4404.

[55] Nicola L, Van der Giessen E, Needleman A. Two hardening mechanisms in single crystal thin films studied by discrete dislocation plasticity. Philosophical Magazine 2005;85:1507.

[56] Nicola L, Van der Giessen E, Needleman A. Size effects in polycrystalline thin films analyzed by discrete dislocation plasticity. Thin Solid Films 2005;479:329.

[57] Nicola L, Xiang Y, Vlassak JJ, Van der Giessen E, Needleman A. Plastic deformation of freestanding thin films: Experiments and modeling. Journal of the Mechanics and Physics of Solids 2006;54:2089.

[58] Hartmaier A, Buehler MJ, Gao HJ. Multiscale modeling of deformation in polycrystalline thin metal films on substrates. Advanced Engineering Materials 2005;7:165.

[59] Han CS, Hartmaier A, Gao HJ, Huang YG. Discrete dislocation dynamics simulations of surface induced size effects in plasticity. Materials Science and Engineering a-Structural Materials Properties Microstructure and Processing 2006;415:225.

[60] Pant P, Schwarz KW, Baker SP. Dislocation interactions in thin FCC metal films. Acta Materialia 2003;51:3243.

[61] von Blanckenhagen B, Arst E, Gumbsch P. Discrete dislocation simulation of plastic deformation in metal thin films. Acta Materialia 2004;52:773.

[62] Espinosa HD, Berbenni S, Panico M, Schwarz KW. An interpretation of size-scale plasticity in geometrically confined systems. Proceedings of the National Academy of Sciences of the United States of America 2005;102:16933. 
[63] Espinosa HD, Panico M, Berbenni S, Schwarz KW. Discrete dislocation dynamics simulations to interpret plasticity size and surface effects in freestanding FCC thin films. International Journal of Plasticity 2006;22:2091.

[64] Fertig Iii RS, Baker SP. Dislocation dynamics simulations of dislocation interactions and stresses in thin films. Acta Materialia 2010;58:5206.

[65] Kim J-Y, Greer JR. Tensile and compressive behavior of gold and molybdenum single crystals at the nano-scale. Acta Materialia 2009;57:5245. 


\section{CHAPTER 2}

\section{DISLOCATION DYNAMICS SIMULATIONS}

In recent years, dislocation dynamics (DD) simulations have attracted lots of interest because of its power to simulate materials deformations and study the plastic flow in crystalline materials [1-12]. There are several versions of 3D DD SIMULATIONS around the world, most of which represent dislocation loops as many straight or curved segments based on single dislocation theory and simulate the collective behavior of dislocation ensembles. The parametric dislocation dynamics (PDD) [9-10] developed by Ghoniem and colleagues has been employed in our studies, which avoided the abrupt variation or singularities associated with the self-force at the joining nodes in between segments and easily handled drastic variations in dislocation curvature without excessive re-meshing. In this chapter, the formulation of PDD is briefly introduced and summarized.

\subsection{The displacement field of dislocations in isotropic crystals}

A dislocation is formed by making a hypothetical cut through a sold piece of material, followed by rigid translation of the negative side of $\left(S^{-}\right)$, while holding the positive side $\left(S^{+}\right)$ fixed, as illustrated in Figure 2.1. Define the dislocation line vector $\mathbf{t}$ as the tangent to the dislocation line. The Burgers vector $b$ is prescribed as the displacement jump condition across the surface $(S)$. The elastic field is based on the Burgers equation [13], which defines the distribution of elastic displacements around dislocation loops. Referring to Figure 2.1, we define the dislocation loop by cutting over the surface $S$ and translating the negative side by the vector $\mathbf{b}$, while holding the positive side fixed. Along any linking curve $\gamma$, the closed line integral of the displacement vector is $\mathbf{b}$. Thus, 


$$
b_{i}=\oint_{\gamma} u_{i, j} d x_{j}
$$

For a given force distribution $f_{m}(\widehat{r})$ in the medium, the displacement vector is given by

$$
u_{k}(\mathbf{r})=\int_{\text {all space }} G_{k m}(\mathbf{r}-\hat{\mathbf{r}}) f_{m}(\hat{\mathbf{r}}) d^{3} \hat{\mathbf{r}}
$$

where $G_{k m}(\mathbf{r}-\hat{\mathbf{r}})=G_{k m}(\mathbf{R})$ are the isotropic elastic Green's functions,

$$
G_{k m}(\mathbf{R})=\frac{1}{8 \pi \mu}\left[\delta_{k m} R_{, p p}-\left(\frac{\lambda+\mu}{\lambda+2 \mu}\right) R_{, k m}\right]
$$

Here $\mu$ and $\lambda$ are Lamé constants, and $\delta_{i j}$ is Kronecker delta. For the volume $V$, bound by the surface $S$, and upon utilization of the divergence theorem for any rank tensor $\mathbf{T}$

$$
\int_{V} \mathbf{T}_{, i} d V=\int_{S} \mathbf{T} d S_{i}
$$

We obtain

$$
\begin{aligned}
u_{m}(\mathbf{r})= & \int_{V} G_{i m}(\mathbf{r}-\hat{\mathbf{r}}) f_{i}(\hat{\mathbf{r}}) d V-\int_{S} u_{i}(\hat{\mathbf{r}}) C_{i j k l} G_{k m, l}(\mathbf{r}-\hat{\mathbf{r}}) d S_{j} \\
& +\int_{S} u_{i}(\hat{\mathbf{r}}) C_{i j k l} G_{k m, l}(\mathbf{r}-\hat{\mathbf{r}}) d S_{j}
\end{aligned}
$$

The second and third terms in eq. (2.5) account for displacement and traction boundary conditions on the surface $S$, respectively. Assuming that body forces are absent in the medium, as well as zero traction and rigid displacements $b_{i}$ across the surface $S$, we obtain

$$
u_{m}(\mathbf{r})=-b_{i} \int_{S} C_{i j k l} G_{k m, l}(\mathbf{r}-\hat{\mathbf{r}}) d S_{j}
$$



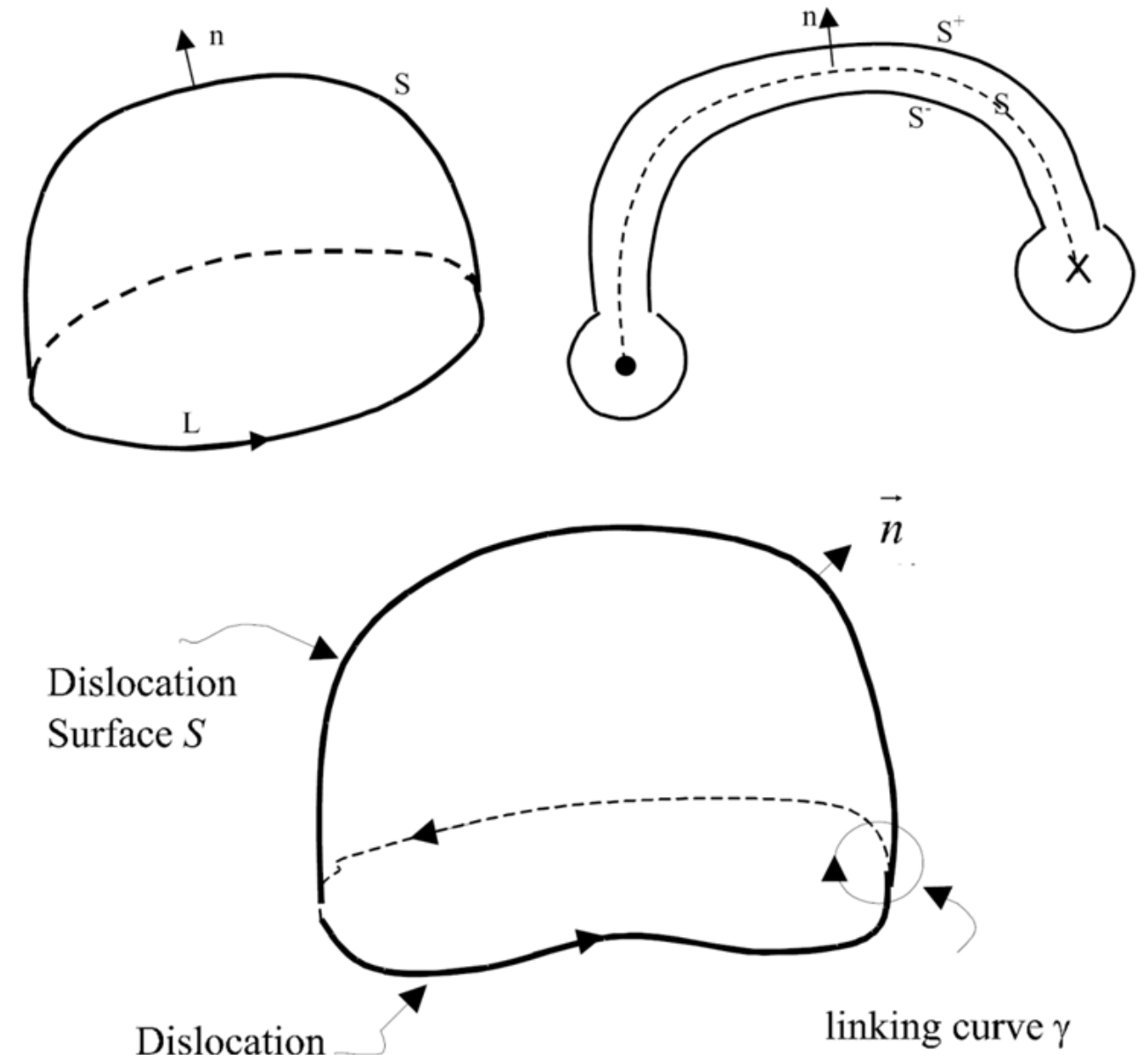

line $C$

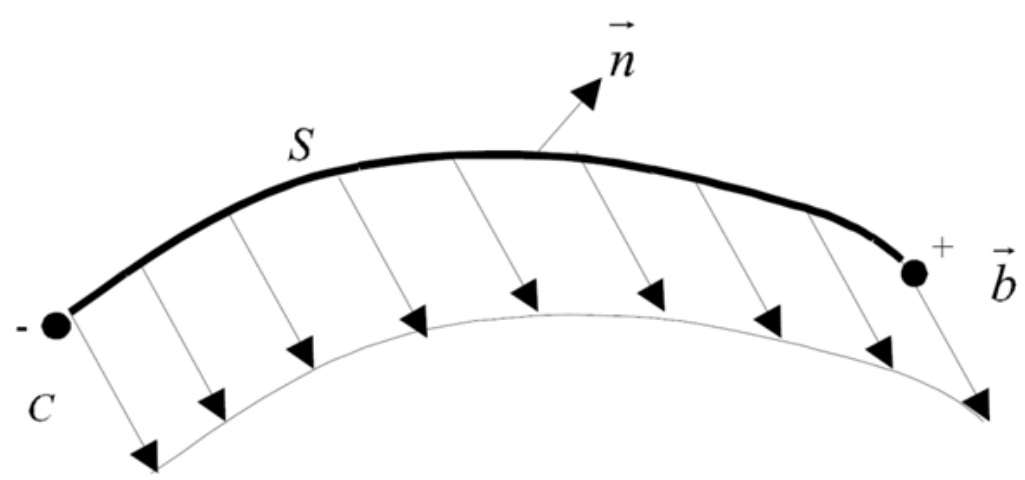

Figure 2.1 Creation of a dislocation by a cut on the surface $(S)$ [9]. 
For an elastic isotropic medium, the fourth-rank elastic constant tensor is given in terms of Lamé constants $\mu$ and $\lambda$, and thus $C_{i j k l}=\mu\left(\delta_{i k} \delta_{i k}+\delta_{i k} \delta_{i k}\right)+\lambda \delta_{i j} \delta_{k l}$. Substituting in eq. (2.6) and rearranging terms, the displacement vector is given by

$$
\begin{aligned}
u_{m}(\mathbf{r})= & \frac{1}{8 \pi} \int_{S} b_{m} R_{, p p j} d S_{j}+\frac{1}{8 \pi} \int_{S}\left(b_{l} R_{, p p l} d S_{m}-b_{j} R_{, p p m} d S_{j}\right) \\
& +\frac{1}{4 \pi} \frac{\lambda+\mu}{\lambda+2 \mu} \int_{S}\left(b_{i} R_{, p p m} d S_{j}-b_{k} R_{, k m j} d S_{j}\right)
\end{aligned}
$$

Equation 2.7 can be converted to a line integral through Stokes' theorem:

$$
u_{i}=-\frac{b_{i} \Omega}{4 \pi}+\frac{1}{8 \pi} \oint_{C}\left[\varepsilon_{i k l} b_{l} R_{, p p}+\frac{1}{1-v} \varepsilon_{k m n} b_{n} R_{, m i}\right] d l_{k}
$$

where $\varepsilon_{i j k}$ is permutation tensor and $\Omega$ is the solid angle formed by the point of interest with respect to the dislocation line. As shown in Figure 2.2, the solid angle differential $d \Omega$ is the ratio of the projected area element $d \mathbf{S}$ to the square of $R$. Thus:

$$
\Omega=\int d \Omega=\int_{S} \frac{\mathbf{e} \bullet d \mathbf{S}}{R^{2}}=\int_{S} \frac{X_{i} d S_{i}}{R^{3}}=-\frac{1}{2} \int_{S} R_{, p p i} d S_{i}
$$

where $\mathbf{e}=\mathbf{R} / R=\operatorname{set}\left\{e_{i}\right\}$ is a unit vector along $\mathbf{R}=$ set $\left\{X_{i}\right\}$, and $R_{\text {,ppi }}=-2 X_{i} / R^{3}$. The solid angle can be computed as a line integral, by virtue of Stokes theorem. Taking the derivatives of $\Omega$ in eq. (2.9), and applying Stokes theorem, we obtain:

$$
\Omega_{, i}=\frac{1}{2} \int_{S}\left(R_{, p p l} d S_{j}-R_{, p p l} d S_{l}\right)=\frac{1}{2} \oint_{C} \varepsilon_{j k l} R_{, p p l} d l_{k}
$$

Thus, the displacement field of a single dislocation loop could be determined by eq. (2.8). 


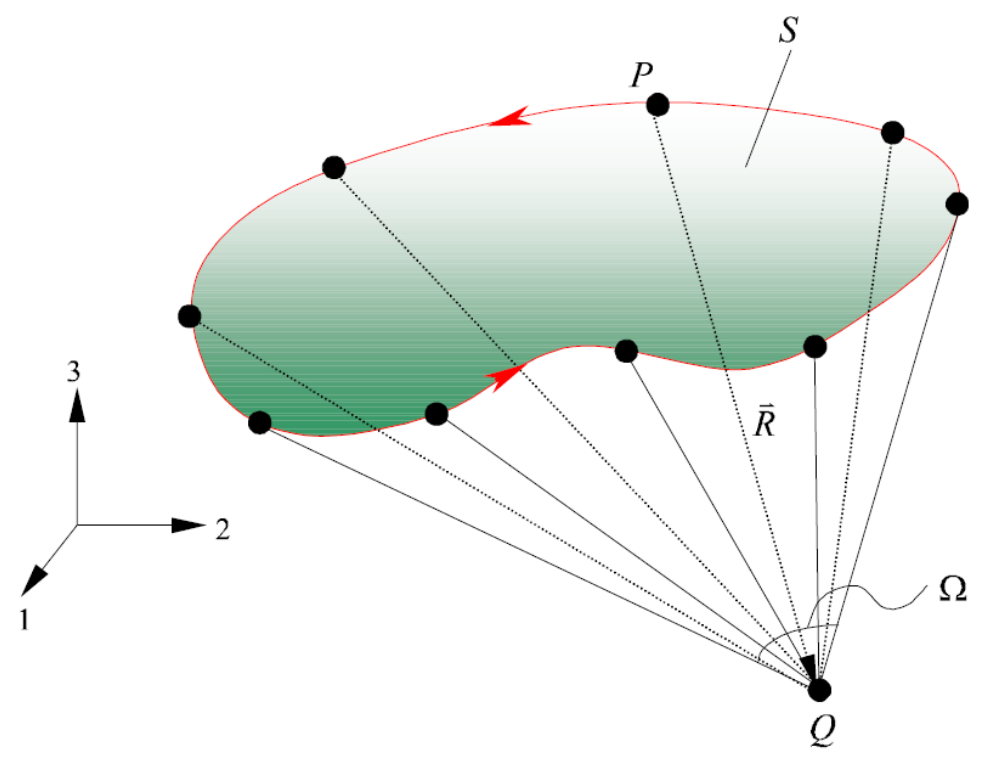

Figure 2.2 Representation of the solid angle, $\Omega$, at a field point $(Q)$ away from the dislocation loop line containing the set of points $(P)[10]$.

\subsection{Strain and stress fields}

Once the displacement field is determined, the strain tensor can be obtained from deformation gradients, while the stress tensor is readily accessible through linear constitutive relations. If we denote the deformation gradient tensor by $u_{i j}$, the strain tensor $\varepsilon_{i j}$ in infinitesimal elasticity is its symmetric decomposition:

$$
u_{i j}=\frac{1}{2}\left(u_{i, j}+u_{j, i}\right)+\frac{1}{2}\left(u_{i, j}-u_{j, i}\right)=\varepsilon_{i j}+\omega_{i j}
$$

where $\omega_{i j}$ is the rotation tensor. Taking the derivatives of eq. (2.11) yields the deformation gradient tensor 


$$
u_{i, j}=-\frac{b_{i} \Omega_{, i}}{4 \pi}+\frac{1}{8 \pi} \oint_{C}\left[\varepsilon_{i k l} b_{l} R_{, p p i}+\frac{1}{1-v} \varepsilon_{k m n} b_{n} R_{, m i j}\right] d l_{k}
$$

from which the following strain tensor is obtained

$$
\varepsilon_{i j}=-\frac{b_{i} \Omega_{, j}+b_{j} \Omega_{, i}}{8 \pi}+\frac{1}{8 \pi} \oint_{C}\left[\frac{1}{2}\left(\varepsilon_{j k l} b_{l} R_{, i}+\varepsilon_{i k l} b_{l} R_{, j}\right)_{, p p}+\frac{\varepsilon_{k m n} b_{n} R_{, m i j}}{1-v}\right] d l_{k}
$$

The derivatives of the solid angle $\Omega$ are given by eq. (2.10), which can be used to derive the strain tensor components as line integrals

$$
\varepsilon_{i j}=\frac{1}{8 \pi} \oint_{C}\left[\frac{1}{2}\left(\varepsilon_{i k l} b_{l} R_{, j}+\varepsilon_{j k l} b_{l} R_{, i}-\varepsilon_{j k l} b_{i} R_{, l}-\varepsilon_{i k l} b_{j} R_{, l}\right)_{, p p}+\frac{\varepsilon_{k m n} b_{n} R_{, m i j}}{1-v}\right] d l_{k}
$$

To deduce the stress tensor, we use the isotropic stress-strain relations of linear elasticity.

First, the dilatation is obtained by letting both $i$ and $j=r$ in eq. (2.14)

$$
\varepsilon_{r r}=-\frac{1}{8 \pi} \frac{1-2 v}{1-v} \oint_{C} \varepsilon_{k m n} b_{n} R_{, m r r} d l_{k}
$$

Using the stress-strain relations $\sigma_{i j}=2 \mu \varepsilon_{i j}+\lambda \varepsilon_{r r} \delta_{i j}$, we can readily obtain the stress tensor

$$
\sigma_{i j}=\frac{\mu b_{n}}{4 \pi} \oint_{C}\left[\frac{1}{2} R,_{m p p}\left(\varepsilon_{j m m} d l_{i}+\varepsilon_{i m n} d l_{j}\right)+\frac{1}{1-v} \varepsilon_{k m n}\left(R,{ }_{i j m}-\delta_{i j} R,{ }_{p p m}\right) d l_{k}\right] .
$$




\subsection{Self force of dislocations}

Once the stress and strain tensors are found, elastic self-energy can be obtained. By considering an infinitesimal variation in the position of the dislocation loop over a time interval $\delta t$, an expression for the sefl-energy of the loop can be formulated. This formulation is developed by Gavazza and Barnet and presented by Ghoniem is given as a single line integral over the dislocation loop $C$

$$
\begin{aligned}
U_{\text {self }} & =\int_{\Omega}\left[\sigma_{i j} d \varepsilon_{i j}\right]^{(s)} d \Omega=\oint_{C} f(\kappa, \alpha, \varphi) \bullet d r \\
& =\oint_{C}\left(\left[E(\mathbf{t})-\left(E(\mathbf{t})+E^{\prime \prime}(\mathbf{t})\right) \ln \left(\frac{8}{\varphi \kappa}\right)\right] \kappa-J(L, \mathbf{P})\right) \mathbf{n} \bullet \delta \mathbf{r}|d s|+[\delta U]_{\text {core }}
\end{aligned}
$$

where $\mathbf{n}$ is normal to the dislocation line vector $\mathbf{t}$ on the glide plane, and $\varphi=|\mathbf{b} / 2|$ is the dislocation core radius [14]. The first term results from loop stretching during the infinitesimal motion, the second and third are the line tension contribution, while $J(L, \mathbf{P})$ is a non-local contribution to the self-energy. The dominant contributions to the self-energy (or force) are dictated by the local curvature $\kappa$, and contain the pre-logarithmic energy term $E(\mathbf{t})$ for a straight dislocation tangent to the loop at point $\mathbf{P}$, and its second angular derivative $E^{\prime \prime}(\mathbf{t}) .[\delta U]_{\text {core }}$ is the contribution of the dislocation core to the self-energy. Defining the angle

between the Burgers vector and the tangent as $\alpha=\cos ^{-1}\left(\frac{t \cdot b}{|b|}\right)$, Gore [15] showed that a convenient form of the self-energy integral for an isotropic elastic medium of $v=1 / 3$ can be written as

$$
\begin{aligned}
U_{\text {self }}= & \oint_{C}\left\{-\kappa\left[E(\alpha)+E^{\prime \prime}(\alpha)\right] \ln \left(\frac{8}{\varphi \kappa}\right)\right. \\
& \left.+\mu b^{2}\left[\kappa\left(\frac{21+\cos ^{2} \alpha}{64 \pi}\right)+\bar{\kappa}\left(\frac{2 \cos ^{2} \alpha-1}{2 \pi}\right)\right]\right\} \mathbf{n} \bullet \delta \mathbf{r}|d s|
\end{aligned}
$$


where the energy prefactors are given by $E(\alpha)=\left[\mu b^{2} / 4 \pi(1-v)\right]\left(1-v \cos ^{2} \alpha\right), E^{\prime \prime}(\alpha)$ is its second angular derivative, and $\bar{\kappa}$ is the average curvature of the loop.

The self force can be thought of as line tension in the dislocation loop. The direction of this force is directed in, towards the center of curvature of the loop. The self force per unit length is found as follows

$$
\frac{\mathbf{F}}{L}=\frac{\partial \frac{U}{L}}{\partial \mathbf{r}}=-\kappa\left[E(\alpha)+E^{\prime \prime}(\alpha)\right] \ln \left(\frac{8}{\varphi \kappa}\right)+\mu b^{2}\left[\kappa\left(\frac{21+\cos ^{2} \alpha}{64 \pi}\right)+\bar{\kappa}\left(\frac{2 \cos ^{2} \alpha-1}{2 \pi}\right)\right] \mathbf{n}
$$

\subsection{Parametric dislocations}

3-D dislocation loop can be reduced to a continuous line. The parametric dislocation is different from other methods that represent dislocation poops am many straight segments $[3-8,11-12]$. In this method, the dislocation line is segmented into $\left(n_{s}\right)$ arbitrary curved segments, labeled $\left(1 \leq j \leq n_{s}\right)$, as shown in Figure 2.3(a). For each segment, we define $\mathbf{P}(u)$ as the position vector for any point on the segment, $\mathbf{T}(u)=T \mathbf{t}$ as the tangent vector to the dislocation line. The space curve is then completely described by the parameter $u$, if one defines certain relationships which determine $\mathbf{P}(u)$. As shown in Figure 2.3(b), segment $j$ is expressed as a function of a variable $\mathrm{u}$ which is from 0 to 1 , and the positions of two dislocation nodes:

$$
\mathbf{P}^{(j)}(u)=\sum_{i=0}^{N_{D F}} C_{i}(u) \mathbf{q}_{i}
$$

where $N_{D F}$ is the number of total generalized coordinates at two ends of the loop segment, $C_{i}(u)$ are the general shape functions, $\mathbf{q}_{i}$ are general coordinates of dislocation nodes. 


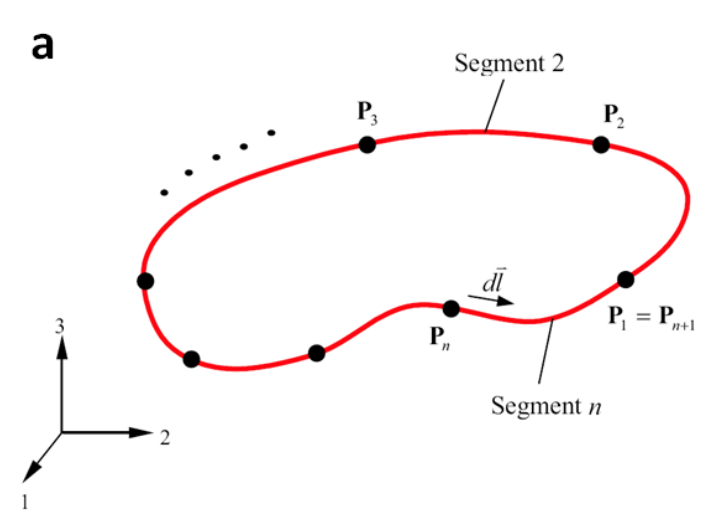

b

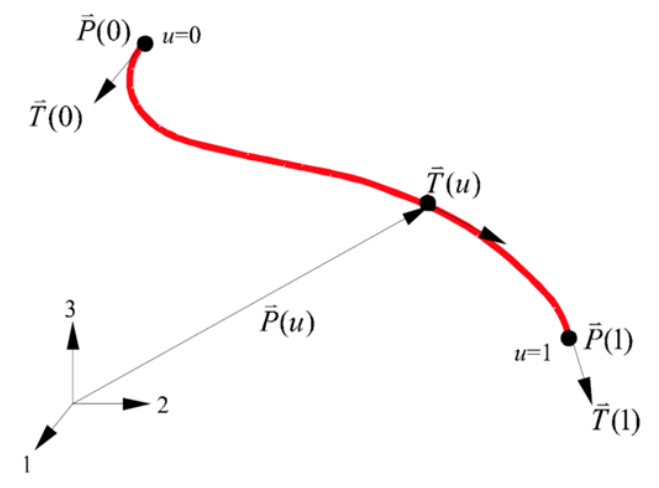

Figure 2.3 Parametric representation of dislocation lines. (a) A dislocation loop is divided into segments connecting dislocation nodes; (b) a curved dislocation segment between two nodes [10].

In PDD used in this thesis, dislocation loops are divided into segments that are represented as cubic spline curves, which could approximates self-force on a dislocation as a simple function of its curvature and allow for continuity of the self-force along the entire dislocation loop to capture non-linear deformations of the dislocation line itself. For cubic spline segments, we use the following set of shape functions and their associated degrees of freedom, respectively:

$$
\begin{aligned}
& C_{1}(u)=2 u^{3}-3 u^{2}+1 \\
& C_{2}(u)=-2 u^{3}+3 u^{2} \\
& C_{3}(u)=u^{3}-2 u^{2}+u \\
& C_{4}(u)=u^{3}-u^{2} \\
& \mathbf{q}_{1}=\mathbf{P}^{(j)}(0) \\
& \mathbf{q}_{2}=\mathbf{P}^{(j)}(1) \\
& \mathbf{q}_{3}=\mathbf{T}^{(j)}(0)
\end{aligned}
$$




$$
\mathbf{q}_{4}=\mathbf{T}^{(j)}(1)
$$

where $\mathbf{P}^{(j)}(0)$ and $\mathbf{T}^{(j)}(0)$ are position and tangent vectors of the beginning point of segment $j$ where $u=0$, and $\mathbf{P}^{(j)}(1)$ and $\mathbf{T}^{(j)}(1)$ are the position and tangent vectors of the ending point of segment $j$ where $u=1$.

Following section 2.2, the strain field and stress filed tensors at any point due to $N_{\text {loop }}$ dislocation loops that are divided into $N_{\text {seg }}$ segments can be written as fast numerical sum over: quadrature points $\left(1 \leq \alpha \leq Q_{\max }\right)$ associated with weighting factors $\left(\omega_{\alpha}\right)$, loop segments $\left(1 \leq \beta \leq N_{s}\right)$, and number of ensemble loops $\left(1 \leq \gamma \leq N_{\text {loop }}\right)[9]$ :

$$
\begin{aligned}
\varepsilon_{i j}= & \frac{1}{8 \pi} \sum_{\gamma=1}^{N_{\text {loop }}} \sum_{\beta=1}^{N_{s}} \sum_{\alpha=1}^{Q_{\max }} \omega_{\alpha}\left(\frac{\varepsilon_{k m n} b_{n} R_{, m i j}}{1-v}\right. \\
& \left.+\frac{1}{2}\left(\varepsilon_{i k l} b_{l} R_{, j}+\varepsilon_{j k l} b_{l} R_{, i}-\varepsilon_{j k l} b_{i} R_{, l}-\varepsilon_{i k l} b_{j} R_{, l}\right)_{, p p}\right) x_{k, u} \\
\sigma_{i j}= & \frac{\mu}{4 \pi} \sum_{\gamma=1}^{N_{\text {loop }}} \sum_{\beta=1}^{N_{s}} \sum_{\alpha=1}^{Q_{\max }} b_{n} \omega_{\alpha}\left[\frac{1}{2} R_{, m p p}\left(\varepsilon_{j m m} x_{i, k}+\varepsilon_{i m n} x_{j, u}\right)\right. \\
& \left.+\frac{1}{1-v} \varepsilon_{k m n}\left(R_{, i j m}-\delta_{i j} R,{ }_{p p m}\right) x_{k, u}\right]
\end{aligned}
$$

\subsection{Equations of motion}

A derivation based on thermodynamics has been developed to obtain a variational form for the equations of motion (EOM) for dislocation loops [9, 16]. The effects of inertia on dislocation motion can become important under conditions of very high strain rates, such as during shock propagation. In most other situations including the cases in this thesis, inertia can be safely ignored. This means that, to a good approximation, there is no need to worry 
about the acceleration and masses of the dislocations. Motion in this regime is often called over-damped motion, where the force determines the instantaneous velocity, leading to a first-order differential equation of motion:

$$
\oint_{C}\left(f_{k}^{t}(t)-B_{\alpha k} V_{\alpha}\right) \delta r_{k}|d s|=0
$$

where $B_{\alpha k}$ is the resistive matrix which is related to the mobility of dislocations, $\mathrm{V}_{\alpha}$ is the velocity of dislocations, and $\mathbf{f}^{t}=\mathbf{f}_{S}+\mathbf{f}_{O}+\mathbf{f}_{P K}$ is the total force acting on the dislocations and is summation of the self-force $\mathbf{f}_{S}$ of dislocations, the osmotic force $\mathbf{f}_{O}$ induced by nonequilibrium point defects on dislocations [17] and the Peach-Koehler force $\mathbf{f}_{P K}$, which can be written as:

$$
\mathbf{f}_{P K}=\left(\left(\boldsymbol{\sigma}_{a p p}+\boldsymbol{\sigma}_{\text {int }}+\boldsymbol{\sigma}_{i m g}\right) \cdot \mathbf{b}\right) \times \mathbf{t}
$$

where $\mathbf{b}$ is the burgers vector of dislocations, $t$ is the tangent vector of the dislocation lines, $\boldsymbol{\sigma}_{\text {app }}$ is the applied stress field, $\boldsymbol{\sigma}_{\text {int }}$ is the stress field from interaction of dislocations and $\boldsymbol{\sigma}_{\text {img }}$ is the image stress field described in Chapter 3.

Suppose that the dislocation line is divided into $N_{s}$ segments, by applying the Galerkin method and using the fat-sum strategy [10], the equation of motion (2.24) can be written as:

$$
F_{k}=\sum_{l=1}^{N_{\text {total }}} \Gamma_{k l} Q_{l, t}
$$

where $\left[F_{k}\right]$ is the general force load, $\left[\Gamma_{k l}\right]$ is the general resisitivity matrix and $\left[Q_{l, t}\right]$ is the general coordinates of dislocation nodes. By solving this equation dislocation positions are obtained.

\subsection{Simulation procedure}


The simulation procedure for a typical DD simulation is illustrated in this section, which is used for a uniaxial load applied to the deformation of materials. The corresponding microstructure evolution and the stress-strain curve are obtained from the results.

The loading is applied through a constant strain rate. Define

$$
c=\dot{\varepsilon}^{e}+\dot{\varepsilon}^{p}
$$

as the applied strain rate, where $\dot{\varepsilon}^{e}$ is the elastic strain rate and $\dot{\varepsilon}^{p}$ the plastic strain rate. The plastic strain rate is obtained from the motion of dislocations as

$$
\dot{\varepsilon}^{p}=\frac{1}{2 V} \sum_{i=1}^{N_{t o t}} l_{i}^{\alpha} v_{i}^{\alpha}\left(\boldsymbol{b}_{i} \otimes \boldsymbol{n}^{\alpha}+\boldsymbol{n}^{\alpha} \otimes \boldsymbol{b}_{i}\right)
$$

where $V$ is the volume of the simulated crystal, $N_{\text {tot }}$ is the total number of dislocation

segments, $l_{i}^{\alpha}$ is the length of dislocation segment $i$ moving on the slip plane $\alpha$, and $v_{i}^{\alpha}$ is the corresponding moving velocity of the segment $i . \quad \boldsymbol{b}_{i}$ and $\boldsymbol{n}^{\alpha}$ are the Burgers vector of dislocation segment $i$ and the normal of slip plane $\alpha$, respectively.

The elastic strain rate is defined as:

$$
\dot{\varepsilon}^{e}=\frac{\dot{\sigma}}{E}
$$

where $E$ is the Young's modules.

Substituting eq. 2.27 and 2.28 into eq. 2.26, we can obtain the expression of the relation of applied stress and strain rate as

$$
\dot{\sigma}=E\left(c-\dot{\varepsilon}^{p}\right)
$$




$$
\begin{aligned}
& \text { Because of } \dot{\sigma}=\frac{\sigma^{t+1}-\sigma^{t}}{\delta t} \text {, eq. } 2.29 \text { leads to } \\
& \qquad \sigma^{t+1}=\sigma^{t}+E \delta t\left(c-\dot{\varepsilon}^{p}\right)
\end{aligned}
$$

where $\delta t$ is the time step.

In simulations, the plastic strain rate is calculated from the motion of dislocations. Using eq. 2.30 we can obtain the strain-stress curves. The simulations will directly relate the dislocation motion at the microscale to the macroscale mechanical properties, which provides a way to study the material behaviors. 


\section{References}

[1] Kubin LP, Canova G. The modeling of dislocation patterns. Scripta Metallurgica Et Materialia 1992;27:957.

[2] Devincre B, Condat M. Model validation of a 3d simulation of dislocation dynamics discretization and line tension effects. Acta Metallurgica Et Materialia 1992;40:2629.

[3] Devincre B, Kubin LP. Simulations of forest interactions and strain-hardening in fcc crystals. Model. Simul. Mater. Sci. Eng. 1994;2:559.

[4] Fivel M, Verdier M, Canova G. 3D simulation of a nanoindentation test at a mesoscopic scale. Materials Science and Engineering a-Structural Materials Properties Microstructure and Processing 1997;234:923.

[5] Rhee M, Zbib HM, Hirth JP, Huang H, de la Rubia T. Models for long-/short-range interactions and cross slip in 3D dislocation simulation of BCC single crystals. Model. Simul. Mater. Sci. Eng. 1998;6:467.

[6] Schwarz KW. Simulation of dislocations on the mesoscopic scale. I. Methods and examples. J. Appl. Phys. 1999;85:108.

[7] Schwarz KW. Local rules for approximating strong dislocation interactions in discrete dislocation dynamics. Model. Simul. Mater. Sci. Eng. 2003;11:609.

[8] Zbib HM, Rhee M, Hirth JP. On plastic deformation and the dynamics of 3D dislocations. International Journal of Mechanical Sciences 1998;40:113.

[9] Ghoniem NM, Sun LZ. Fast-sum method for the elastic field off three-dimensional dislocation ensembles. Physical Review B 1999;60:128.

[10] Ghoniem NM, Tong SH, Sun LZ. Parametric dislocation dynamics: A thermodynamics-based approach to investigations of mesoscopic plastic deformation. Physical Review B 2000;61:913.

[11] Cai W, Bulatov VV, Pierce TG, Hiratani M, Rhee M, Bartelt M, Tang M. Massively-parallel dislocation dynamics simulations. In: Kitagawa H, Shibutani Y, editors. Iutam Symposium on Mesoscopic Dynamics of Fracture Process and Materials Strength, vol. 115. 2004. p.1.

[12] Arsenlis A, Cai W, Tang M, Rhee M, Oppelstrup T, Hommes G, Pierce TG, Bulatov VV. Enabling strain hardening simulations with dislocation dynamics. Model. Simul. Mater. Sci. Eng. 2007;15:553. 
[13] Burgers JM. Some considerations on the fields of stress connected with dislocations in a regular crystal lattice I. Proceedings of the Koninklijke Nederlandse Akademie Van Wetenschappen 1939;42:293.

[14] Dewit R. The continuum theory of stationary dislocations. Solid State Physics Advances in Research and Applications 1960;10:249.

[15] Gore LA. Ph.D. thesis. Stanford University 1980.

[16] Ghoniem NM. Computational methods for mesoscopic, inhomogeneous plastic deformation. Singapore: World Scientific Publ Co Pte Ltd, 2000.

[17] R. W. Balluffi, S. M. Allen, Carter WC. The Boundary Element Method in Engineering: A Complete Course Wiley-Interscience, 2005. 


\section{CHAPTER 3}

\section{IMAGE STRESSES IN DISLOCATION DYNAMICS SIMULATIONS}

In finite volume, free surface boundary condition plays an important role on the plastic deformations, since dislocation motions are limited by the confined geometries. DD SIMULATIONS at small scales mush solve the coupling between surface effect on dislocations and surface deformation caused by dislocation loop evolution. In 2D problems, this process is relatively easy as dislocations are either infinite, semi-infinite straight long, or parallel to the surface face. However, in 3D problems, the surface effects become more complex, because dislocation loops are curved and not necessarily parallel to the free surface.

The formulation and solution of the elastic boundary value problem of dislocations has been pursued by a number of authors in the case of bounded crystals. Van der Giessen and Needleman [1] set up a formalism of this problem based on the principle of superposition in linear elasticity, the total displacement and stress fields are given as

$$
u_{i j}=\tilde{u}_{i j}+\hat{u}_{i j} \text { and } \sigma_{i j}=\tilde{\sigma}_{i j}+\hat{\sigma}_{i j}
$$

where $\tilde{u}_{i j}$ and $\tilde{\sigma}_{i j}$ are the displacement and stress fields in an infinite medium from all dislocations, while $\hat{u}_{i j}$ and $\hat{\sigma}_{i j}$ are the image fields that enforce the boundary conditions. Following this procedure, Fivel and colleagues implemented the Boussinesq-point-force method in DD SIMULATIONS to study the problems containing half space and free-standing thin films [2-3]. Liu and Schwarz successfully evaluated the image field for dislocations intersecting a free surface with high accuracy by Boussinesq-Cerruti formalism. Lemarchand et al. approached the boundary problem in a crystal with a dynamic dislocation configuration by using the discrete-continuum method to solve equilibrium and compatibility conditions in a dislocated material containing interfaces [4]. Khraishi and Zbib employed an 
image segment and a distribution of prismatic rectangular dislocation loops padding the surface to obtain the segment near a free surface is obtained the image stress-field of a subsurface dislocation segment near a free surface [5]. Tang et al. [6] presented a boundary value problem formulation for the image field, in which the singular part of the image field for straight dislocations is analytically considered, while the rest of the image field is computed by applying a non-singular traction term using the finite element method (FEM). Recently, Weinberger and Cai decomposed the traction force on the free surface into Fourier modes by a discrete Fourier transform and obtained the image stress field by superimposing analytic solutions in the Fourier space [7-8].

In all of these works, the stress field of dislocations in bounded domains was evaluated with a reasonable accuracy. However, most of them have to be restricted to the simulation of plastic properties in nano- or submicronic scales containing very few dislocations (although the density can be high). The simulations with samples sizes from half micron to several microns containing free interfaces appear to be out of reach for the moment in above methods. Currently, only two attempts have successfully accomplished this task. One work is done by Weygand and colleagues who presented an approximate solution of the image stress problem in the spirit of combination of the virtual dislocation technique and coarse-meshed FEM [9]. Another method has been developed by El-Awady and coworkers to compute the image stress field of dislocations in micropillars by using boundary element method (BEM) [10].

The BEM belongs to superposition methods and has several advantages over the FEM [11]:

1. Less data preparation time. This is a direct result of the 'surface-only' modelling (i.e. the reduction of dimensionality by one). Thus the analyst's time required for data preparation (and data checking) for a given problem should be greatly reduced. 
2. High resolution of stresses. Stresses are accurate because no further approximation is imposed on the solution at interior points, i.e. solution is exact (and fully continuous) inside the domain.

3. Less computer time and storage. For the same level of accuracy, the BEM uses a lesser number of nodes and elements (but a fully populated matrix) as the level of approximation in the BEM solutions is confined to the surface

In this thesis, image fields of dislocations will be evaluated by coupling the BEM with PDD method.

\subsection{Boundary element method}

The direct boundary element formulation for elastostatics problems can be derived from Bett's reciprocal work theorem [12], which states that work done by the stresses of system $\left(\mathrm{u}_{\mathrm{i}}\right.$, $\left.t_{i}, b_{i}\right)$ on the displacements of system $\left(u_{i}^{*}, t_{i}^{*}, b_{i}^{*}\right)$ is equal to the work done by the stresses of system $\left(u_{i}^{*}, t_{i}^{*}, b_{i}^{*}\right)$ on the displacements of system $\left(u_{i}, t_{i}, b_{i}\right) ; u_{i}$ and $u_{i}^{*}$ are displacements; $t_{i}$ and $t_{i}^{*}$ are tractions; $b_{i}$ and $b_{i}^{*}$ are body forces in the domain $V$ with boundary $S$ :

$$
\int_{S} t_{i} u_{i}^{*} d S+\int_{V} b_{i} u_{i}^{*} d V=\int_{S} t_{i}^{*} u_{i} d S+\int_{V} b_{i}^{*} u_{i} d V
$$

The boundary integral equation for elastostatic problems can be derived by taking the body force $b_{i}^{*}$ to correspond to a point force in an infinite sheet, represented by the Dirac delta function $\Delta(\mathrm{x}-\mathrm{X})$ as

$$
b_{i}^{*}=\Delta(x-X) e_{i}
$$

where the unit vector component $e_{i}$ corresponds to a unit positive force in the $\mathrm{i}$ direction applied at $\mathrm{X}$ and $\mathrm{x}, \mathrm{X} \in \mathrm{V}$. In three-dimensional problems, $\mathrm{e}_{\mathrm{i}}$ is a pure concentrated force. 
The Dirac delta function has the property

$$
\int_{V} g(x) \Delta(x-X) d V=g(X)
$$

Using this property, the last integral in eq. 3.2 can be written as

$$
\int_{V} b_{i}^{*} u_{i} d V=\int_{V} \Delta(x-X) e_{i} u_{i} d V=u_{i}(X) e_{i}
$$

The displacement and traction fields corresponding to the point force solution can be written as

$$
u_{i}^{*}=U_{i, j}(X, x) e_{j}
$$

and

$$
t_{i}^{*}=T_{i, j}(X, x) e_{j}
$$

From the above solutions and eq. (3.3), it can be seen that eq. (3.2) can be written as

$$
\begin{aligned}
u_{i}(X)= & \int_{S} U_{i j}(X, x) t_{j}(x) d S \\
& -\int_{S} T_{i j}(X, x) u_{j}(x) d S+\int_{S} U_{i j}(X, x) b_{j}(x) d V
\end{aligned}
$$

where $\mathrm{x} \in \mathrm{S}$. The above equation is known as the Somigliana's identity for displacements. It relates the value of displacements at an internal point $X$ to boundary values of the displacements and tractions. Equation (3.6) can be written in matrix form for three-dimensional problems as 


$$
\begin{aligned}
& \left\{\begin{array}{l}
u_{1} \\
u_{2} \\
u_{3}
\end{array}\right\}=\int_{S}\left[\begin{array}{lll}
U_{11} & U_{12} & U_{13} \\
U_{21} & U_{22} & U_{23} \\
U_{31} & U_{32} & U_{33}
\end{array}\right]\left\{\begin{array}{l}
t_{1} \\
t_{2} \\
t_{3}
\end{array}\right\} d S \\
& -\int_{S}\left[\begin{array}{lll}
T_{11} & T_{12} & T_{13} \\
T_{21} & T_{22} & T_{23} \\
T_{31} & T_{32} & T_{33}
\end{array}\right]\left\{\begin{array}{l}
u_{1} \\
u_{2} \\
u_{3}
\end{array}\right\} d S+\int_{V}\left[\begin{array}{lll}
U_{11} & U_{12} & U_{13} \\
U_{21} & U_{22} & U_{23} \\
U_{31} & U_{32} & U_{33}
\end{array}\right]\left\{\begin{array}{l}
b_{1} \\
b_{2} \\
b_{3}
\end{array}\right\} d V
\end{aligned}
$$

The strains at any interior point can be obtained by differentiating the displacements in eq. (3.6) with respect to the source point $X$ to give

$$
\begin{aligned}
u_{i, k}(X)= & \int_{S} U_{i j, k}(X, x) t_{j}(x) d S \\
& -\int_{S} T_{i j, k}(X, x) u_{j}(x) d S+\int_{V} U_{i j, k}(X, x) b_{j}(x) d V
\end{aligned}
$$

where $\mathrm{U}_{\mathrm{ij}, \mathrm{k}}$ and $\mathrm{T}_{\mathrm{ij}, \mathrm{k}}$ are derivatives of the fundamental solutions. Finally, Somigliana's identity for stresses can be obtained by substituting eq. (3.8) into Hooke's law $\sigma_{\mathrm{ij}}=$ $2 v \mu /(1-2 v) \delta_{\mathrm{ij}} \varepsilon_{\mathrm{kk}}+2 \mu \varepsilon_{\mathrm{ij}}$, to give

$$
\begin{aligned}
\sigma_{i k}(X)= & \int_{S} D_{k i j}(X, x) t_{k}(x) d S \\
& -\int_{S} S_{k i j}(X, x) u_{k}(x) d S+\int_{V} D_{k i j}(X, x) b_{k}(x) d V
\end{aligned}
$$

where $\mathrm{D}_{\mathrm{kij}}$ and $\mathrm{B}_{\mathrm{kij}}$ are obtained from $\mathrm{U}_{\mathrm{ij}, \mathrm{k}}$ and $\mathrm{T}_{\mathrm{ij}, \mathrm{k}}$, and the application of Hooke's law.

Navier's equation can now be written for a unit point force applied to the body at a point $\mathrm{X}$, as

$$
\mu u_{i, j j}+\frac{\mu}{1-2 v} \mu_{j, j i}+\Delta(x-X) e_{i}=0
$$


The most popular technique for deriving the fundamental solutions is through the use of the Galerkin vector, $G_{i}$. The point force solution in an infinite medium was originally derived by Lord Kelvin, and is known as Kelvin's fundamental solution. The displacements are expressed in terms of the Galerkin vector as

$$
u_{i}=G_{i, k k}-\frac{1}{2(1-v)} G_{k, i k}
$$

Substituting (3.11) into (3.10) gives

$$
u G_{i, k k j j}-\frac{\mu}{2(1-v)} G_{k, i k j j}+\frac{\mu}{(1-v)}\left(G_{j, k k i j}-\frac{1}{2(1-v)}\right) G_{k, j k i j}+\Delta(x-X) e_{i}=0
$$

which can be simplified to

$$
u G_{i, k k j j}+\Delta(x-X) e_{i}=0
$$

since $G_{k, i k j j}=G_{k, j j k i}, G_{j, k k j j}=G_{k, j j k i}$ and $G_{k, j k i j}=G_{k, j j k i}$. Equation (3.13) can also be written as

$$
u \nabla^{2}\left(\nabla^{2} G_{i}\right)+\Delta(x-X) e_{i}=0
$$

The solution of (3.14) is well known from the potential theory and is given by

$$
\nabla^{2} G_{i}=\frac{1}{4 \pi \mu r} e_{i}
$$

for three-dimensional problems. The Galerkin vector is given by

$$
G_{i}=\frac{1}{8 \pi \mu} r e_{i}
$$


Substituting the derivatives of (3.16) into (3.11) gives

$$
u_{i}=\frac{1}{8 \pi \mu}\left[r_{, k k} e_{i}-\frac{1}{2(1-v)} r_{, i k} e_{k}\right]
$$

Now noting that $r_{, i k}=\left(\delta_{i k}-r_{,} r_{, k}\right) / r$ and $r_{, k k}=2 / r$, eq. (3.17) can be rewritten as

$$
u_{i}=\frac{1}{16 \pi \mu(1-v) r}\left[(3-4 v) \delta_{i j}+r_{, i} r_{, j}\right] e_{j}
$$

From (3.4), we have

$$
U_{i j}(X, x)=\frac{1}{16 \pi \mu(1-v) r}\left[(3-4 v) \delta_{i j}+r_{, i} r_{, j}\right]
$$

where $\mathrm{U}_{\mathrm{ij}}(\mathrm{X}, \mathrm{x})$ represents the displacement in the $\mathrm{j}$ direction at point $\mathrm{x}$ due to a unit point force acting in the $\mathrm{i}$ direction at $\mathrm{X}$. The traction fundamental solution is obtained from (3.18), through the usual displacement-strain and strain-stress relationships, and by noting that $\mathrm{t}_{\mathrm{i}}=$ $\sigma_{\mathrm{ji}} \mathrm{n}_{\mathrm{j}}$, to give

$$
\begin{array}{r}
t_{i}=\frac{-1}{8 \pi \mu(1-v) r^{2}\left\{\frac{\partial r}{\partial n}\left[(1-2 v) \delta_{i j}+3 r_{, i} r_{, j}\right]\right.} \\
\left.-(1-2 v)\left(n_{j} r_{, i}-n_{i} r_{j}\right)\right\} e_{j}
\end{array}
$$

where $n_{j}$ denotes the components of the outward normal at the field point x. Again from (3.5), we have 


$$
\begin{aligned}
& T_{i, j}(X, x)=\frac{-1}{8 \pi \mu(1-v) r^{2}}\left\{\frac{\partial r}{\partial n}\left[(1-2 v) \delta_{i j}+3 r_{, i} r_{, j}\right]\right. \\
& \left.-(1-2 v)\left(n_{j} r_{, i}-n_{i} r_{, j}\right)\right\}
\end{aligned}
$$

where $T_{i j}(X, x)$ represents the traction in the $\mathrm{j}$ direction at point $\mathrm{x}$ due to a unit point force acting in the $i$ direction at $X$. Differentiating (3.19) and (3.21) with respect to $X$ gives

$$
\begin{aligned}
U_{i j, k}(X, x)= & \frac{1}{16 \pi \mu(1-v) r^{2}}\left\{(3-4 v) \delta_{i j} r_{, k}+3 r_{, i} r_{, j} r_{, k}-\delta_{j k} r_{, i}-\delta_{k i} r_{, j}\right\} \\
T_{i j, j}(X, x)= & \frac{-1}{8 \pi(1-v) r^{3}}\left\{\left[(1-2 v) \delta_{i j} r_{, k}+3 r_{, i} r_{, j}\right] n_{k}\right. \\
& +(1-2 v)\left[\delta_{j k}-3 r_{, j} r_{, k}\right] n_{i}+(1-2 v)\left[\delta_{k i}-3 r_{, k} r_{, i}\right] n_{j} \\
& \left.+\left[3 \delta_{j k} r_{, i}+3 \delta_{k i} r_{, j}-3(1-2 v) \delta_{i j} r_{, k}-15 r_{, i} r_{, j} r_{, k}\right] r_{, l} n_{, j, k}\right\}
\end{aligned}
$$

Using the stress-strain relationships, gives

$$
D_{k i j}=\frac{1}{8 \pi(1-v) r^{2}}\left[(1-2 v)\left(\delta_{i k} r_{, j}+\delta_{j k} r_{, i}-\delta_{i j} r_{, k}\right)+3 r_{, i} r_{, j} r_{, k}\right]
$$




$$
\begin{aligned}
S_{k i j}= & \frac{\mu}{4 \pi(1-v) r^{3}}\left\{3 \frac{\partial r}{\partial n}\left[(1-2 v) \delta_{i j} r_{, k}+v\left(\delta_{i k} r_{, j}+\delta_{j k} r_{, i}\right)-5 r_{, i} r_{, j} r_{, k}\right]\right. \\
& +3 v\left(n_{i} r_{, j} r_{, k}+n_{j} r_{, i} r_{, k}\right)+(1-2 v)\left(3 n_{k} r_{, i} r_{, j}+n_{j} \delta_{i k}+n_{i} \delta_{j k}\right) \\
& \left.-(1-4 v) n_{k} \delta_{i j}\right\}
\end{aligned}
$$

After we obtained the boundary integral equation relating the displacements and tractions at the surface, divide the surface into elements and use shape functions to describe the geometry and variables over each element. Because analytical integrations are not practical due to the complexity of the integral functions, numerical integration is performed using the Gaussian quadrature technique. Special schemes are necessary to integrate the singular terms when the nodal points are very close to each other or the load point $\mathrm{X}$ coincides with the boundary point $\mathrm{x}$, because the fundamental solution contains terms of the order $(1 / \mathrm{r})$. By summing the integrals over each element, the total surface integral can be evaluated. Form the solution matrix by repeating the integration process with the load point $\mathrm{X}$ placed in turn at each point on the surface, which yields only three equations in three dimensional problems relating all $\mathrm{N}$ variables on the surface, till $3 \mathrm{~N}$ linearly independent equations are formed. The resulting system of linear equations is of the following form:

$$
[\mathrm{A}][\mathrm{u}]=[\mathrm{B}][\mathrm{t}]
$$

Apply the boundary conditions. These take the form of either prescribed displacement or prescribed traction (or stress). By rearranging the linear equations such that all the unknown variables are on the left-hand side and all the known variables are on the right-hand side, the following modified solution matrix is obtained:

$$
\left[\mathrm{A}^{*}\right][\mathrm{x}]=\left[\mathrm{B}^{*}\right][\mathrm{y}]=[\mathrm{c}]
$$


where the unknown vector $[\mathrm{x}]$ contains a mixture of unknown displacements and tractions, while [y] contains all the prescribed values of displacements and tractions. The right-hand side vector [c] is a vector of known coefficients.

Gaussian elimination techniques are used to solve the system of linear equations and compute all boundary displacements and tractions, since the solution matrix is unsymmetric and is fully populated. Then it is straightforward to calculate the displacements and tractions at any interior point of interest. This is achieved by substituting the boundary displacements and traction back into equation (3.8) and (3.9) and solving for the interior point X. When the interior point is close to the boundary the singularity problem will arise. This may be overcome by using integration by parts to transform the nearly singular surface integrals to a series of line integrals along the contour of the elements. In addition, the image force acting on the surface dislocation nodes is simply evaluated by the placing the node under the surface with five Burgers vectors. This cutoff regularization will not affect the dynamical behavior of the dislocations significantly [13], since all fields are computed using continuous dislocations.

In the absence of body forces, the boundary integral equations (3.8) and (3.9), are solved over any closed boundary by dividing the surface into boundary elements, where the integration is obtained numerically over each element. This can be written as a fast numerical sum over: quadrature points $\left(1 \leq \mathrm{n}, \mathrm{s} \leq \mathrm{N}_{\text {Gauss }}\right)$ associated with weight factors $\left(\omega_{\mathrm{n}}\right.$ and $\left.\omega_{\mathrm{s}}\right)$, number of nodes per boundary element $\left(1 \leq \mathrm{c} \leq \mathrm{N}_{\mathrm{n}}\right)$ and number of boundary elements $(1 \leq$ $\left.\mathrm{m} \leq \mathrm{N}_{\mathrm{e}}\right)$ as 


$$
\begin{aligned}
c_{i j}(X) u_{j}(X)= & \sum_{m=1}^{N_{e}} \sum_{c=1}^{N_{n}} \sum_{s=1}^{N_{\text {Gauss }}} \sum_{n=1}^{N_{\text {Gauss }}} w_{n} w_{s} U_{i j}(X, x) N_{c}\left(x_{1}, x_{2}\right) J\left(x_{1}, x_{2}\right) t_{j}(x) \\
& -\sum_{m=1}^{N_{e}} \sum_{c=1}^{N_{n}} \sum_{s=1}^{N_{\text {Gauss }}} \sum_{n=1}^{N_{\text {Gauss }}} w_{n} w_{s} T_{i j}(X, x) N_{c}\left(x_{1}, x_{2}\right) J\left(x_{1}, x_{2}\right) u_{j}(x)
\end{aligned}
$$

and

$$
\begin{aligned}
\sigma_{i j}(X)= & \sum_{m=1}^{N_{e}} \sum_{c=1}^{N_{n}} \sum_{s=1}^{N_{\text {Gauss }}} \sum_{n=1}^{N_{\text {Gauss }}} w_{n} w_{s} D_{k i j}(X, x) N_{c}\left(x_{1}, x_{2}\right) J\left(x_{1}, x_{2}\right) t_{k}(x) \\
& -\sum_{m=1}^{N_{e}} \sum_{c=1}^{N_{n}} \sum_{s=1}^{N_{\text {Gauss }}} \sum_{n=1}^{N_{\text {Gauss }}} w_{n} w_{s} S_{k i j}(X, x) N_{c}\left(x_{1}, x_{2}\right) J\left(x_{1}, x_{2}\right) u_{k}(x)
\end{aligned}
$$

where $c_{\mathrm{ij}}$ is a coefficient matrix, which in general is computed by applying rigid body motion, the functions $\mathrm{N}_{\mathrm{c}}\left(\mathrm{x}_{1}, \mathrm{x}_{2}\right)$ are quadratic shape functions, and the Jacobian of transformation $\mathrm{J}$ is equal to $\sqrt{d x_{1}^{2}+d x_{2}^{2}}$. Thus, the fast sum equations (3.28) and (3.29) are used to calculate the displacements and stresses at all dislocation nodes due to the boundary constraints and are added to the infinite medium solution given by equations (3.1).

\subsection{Numerical results}

In order to determine the accuracy and convergence of the PDD-BEM and optimize the computational time, we compared numerical results to the limiting analytical solutions in the following.

3.2.1 Stress fields associated the edge and screw dislocations 


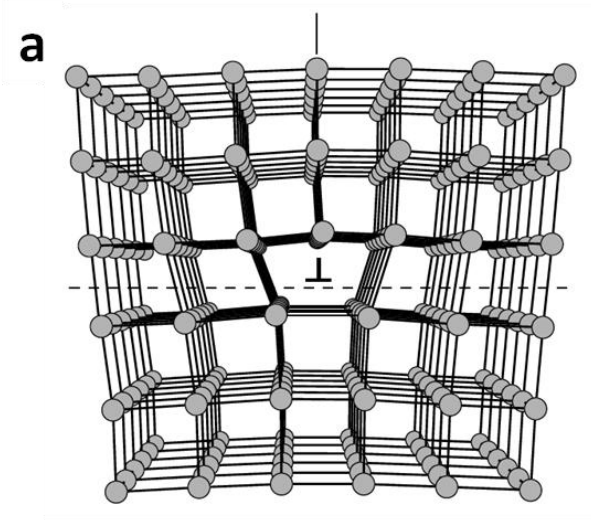

C

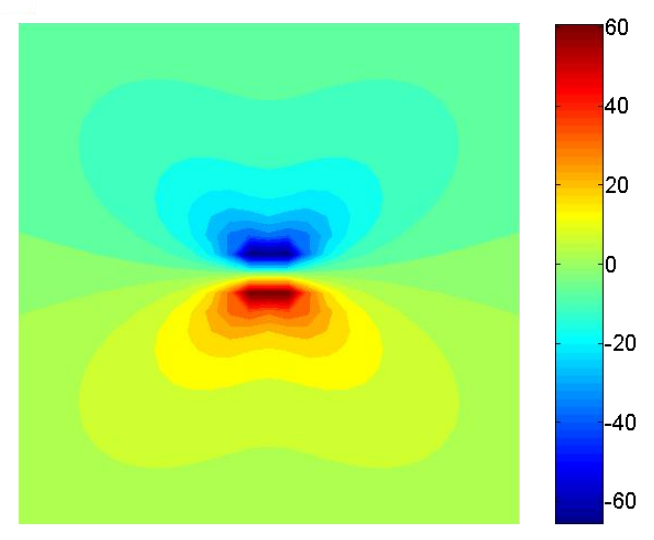

b

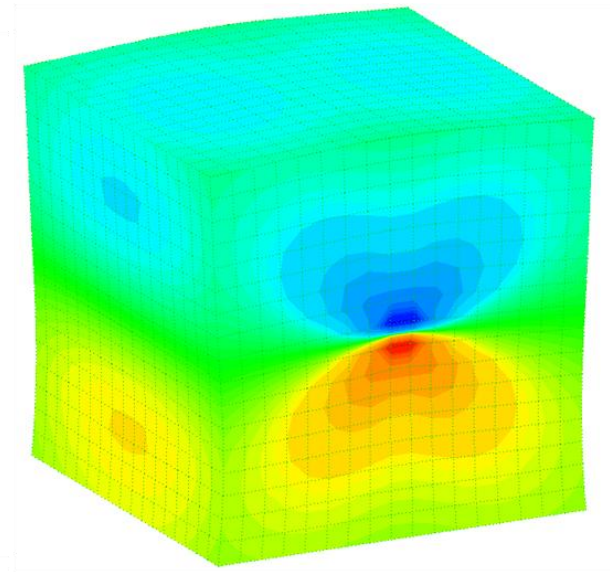

d

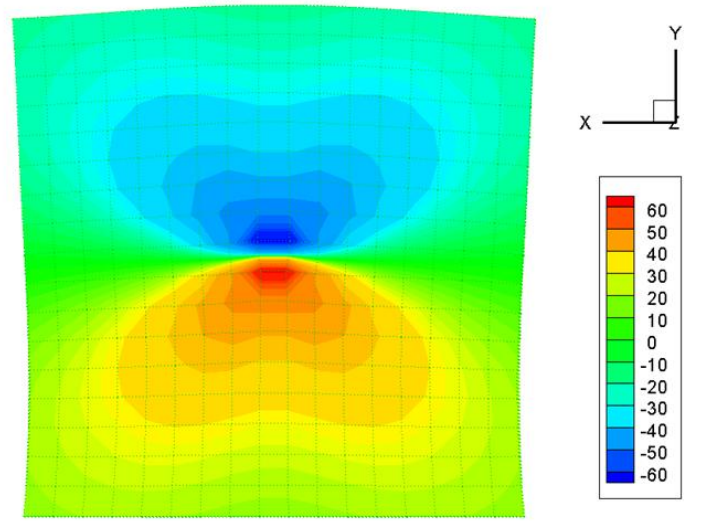

Figure 3.1 Stress and displacement fields associated an edge dislocation, (a) the configuration an edge dislocation created by inserting a half-plane of atoms, (b) 3D view of the stress and displacement field of an edge dislocation from numerical results, (c) the analytical solution of $\sigma \mathrm{xx}$ for an edge dislocation, and (d) the numerical results of $\sigma \mathrm{xx}$ for an edge dislocation (BEM mesh: 1734 elements, displacement magnification: 500, stress unit: MPa).

Since the analytic stress field of dislocation lines under complicated conditions often does not exist, only the stresses fields associated with simple edge and screw dislocations were calculated by the PDD method to compare with analytic solutions.

In the first case, an edge dislocation was set at the middle of a cube with size of $500 \times 500 \times 500 \mathrm{~nm}^{3}$ along Z-direction with slip system $1 / 2[100](010)$ as shown in Figure 3.1, and the analytical solution of $\sigma_{\mathrm{xx}}$ is as following [14]: 


$$
\sigma_{x x}=-\frac{\mu b}{2 \pi(1-v)} \frac{y\left(3 x^{2}+y^{2}\right)}{\left(x^{2}+y^{2}\right)^{2}}
$$

where shear modulus $\mu=50 \mathrm{GPa}$, the magnitude of Burgers vector $\mathrm{b}=0.3 \mathrm{~nm}$, Poisson's ratio $v=0.347$, and $\mathrm{x}, \mathrm{y}$ are the distances to the dislocation in $\mathrm{X}$ and $\mathrm{Y}$ directions.

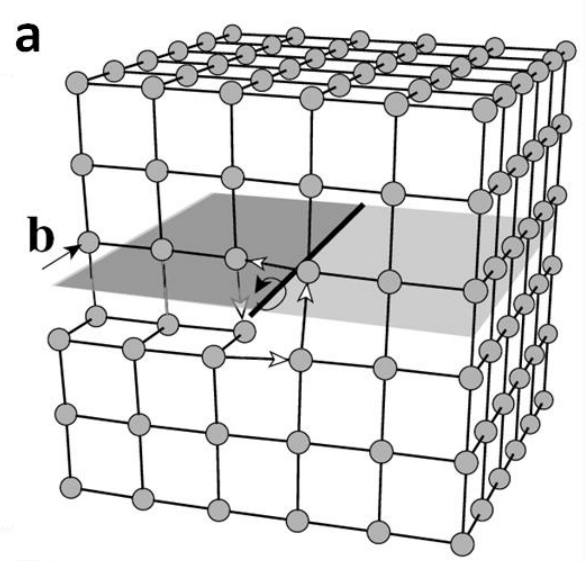

C

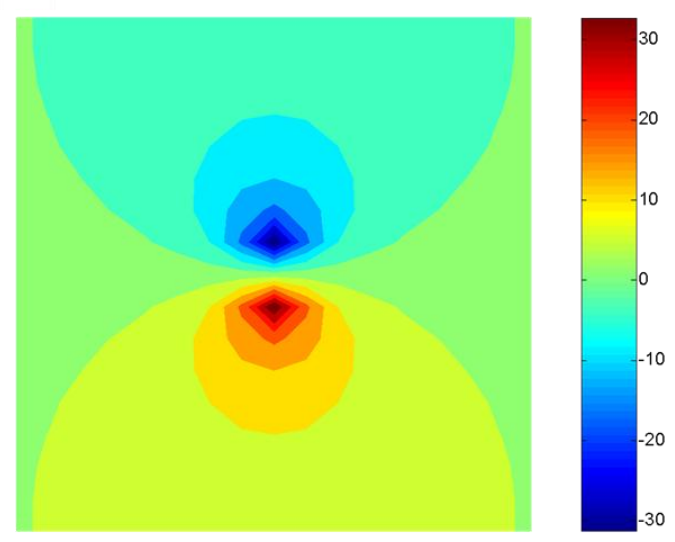

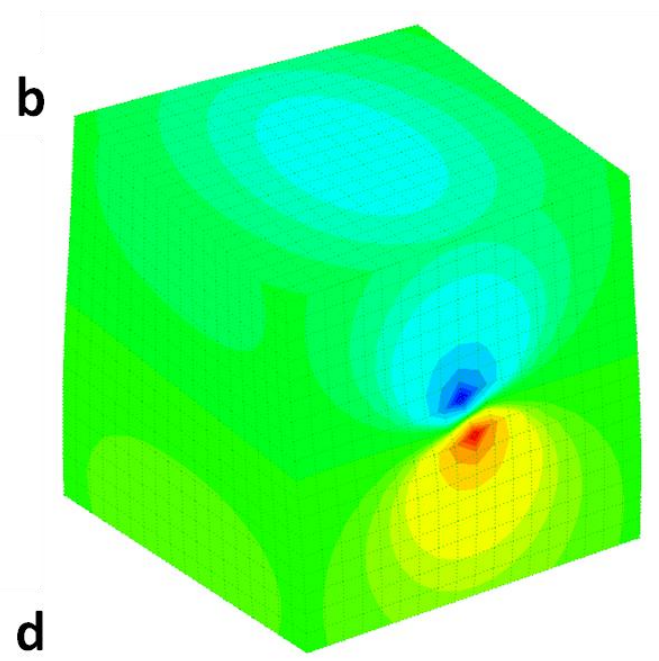

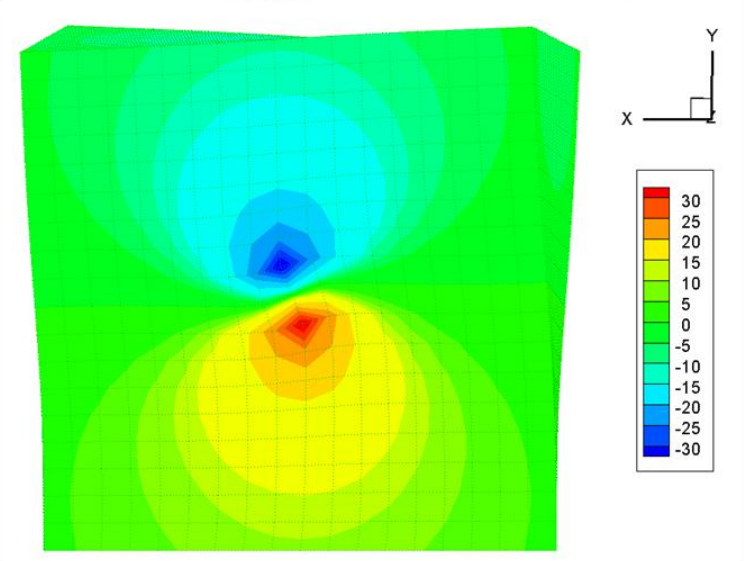

Figure 3.2 Stress and displacement fields associated a screw dislocation, (a) the configuration a screw dislocation created by a "cut-and-slip" procedure in which the slip vector is parallel to the dislocation line, (b) 3D view of the stress and displacement field of a screw dislocation from numerical results, (c) the analytical solution of $\sigma \mathrm{xx}$ for an edge dislocation, and (d) the numerical results of $\sigma x x$ for an edge dislocation (BEM mesh: 1734 elements, displacement magnification: 500, stress unit: MPa). 
In the second case, an screw dislocation was set at the middle of a cube with size of $500 \times 500 \times 500 \mathrm{~nm}^{3}$ along Z-direction with slip system $1 / 2[001](010)$ as shown in Figure 3.2, and the analytical solution of $\sigma_{\mathrm{xx}}$ is as following [14]:

$$
\sigma_{x z}=-\frac{\mu b}{2 \pi} \frac{y}{\left(x^{2}+y^{2}\right)}
$$

As illustrated in Figs. 3.1 and 3.2, the numerical results of magnitudes and distributions of stress field around dislocation lines agree well with the analytic solutions.

\subsubsection{Eshelby twist by a coaxial screw dislocation}

In this case, a coaxial screw dislocation was created in a cylinder of radius $\mathrm{R}=500 \mathrm{~nm}$ and length to diameter ratio of 5:1, to investigate the accuracy and convergence of the PDD-BEM. Eshelby (1953) worked out an analytical solution and predicted that two cross-sections of a cylinder containing a coaxial dislocation [15], will undergo a relative rotation angle $\theta$, given by

$$
\theta=\frac{b L}{\pi R^{2}}
$$

where $\mathrm{L}$ is the distance between the two cross-sections, and $\mathrm{R}$ is the radius of the cylinder. This is the so-called Eshelby twist.

The numerical result computing the deformed shape of a cylinder containing a coaxial screw dislocation was compared with the analytical solution given by eq. (3.32). According to St. Venant's principle, this elementary result can only be used at distances greater than $2 \mathrm{R}$ from the ends. Thus, the two cross-sections are chosen to be located at distances $5 \mathrm{R}$ and $6 \mathrm{R}$ from the bottom edge, respectively. The relative error of the twist angle, for different 

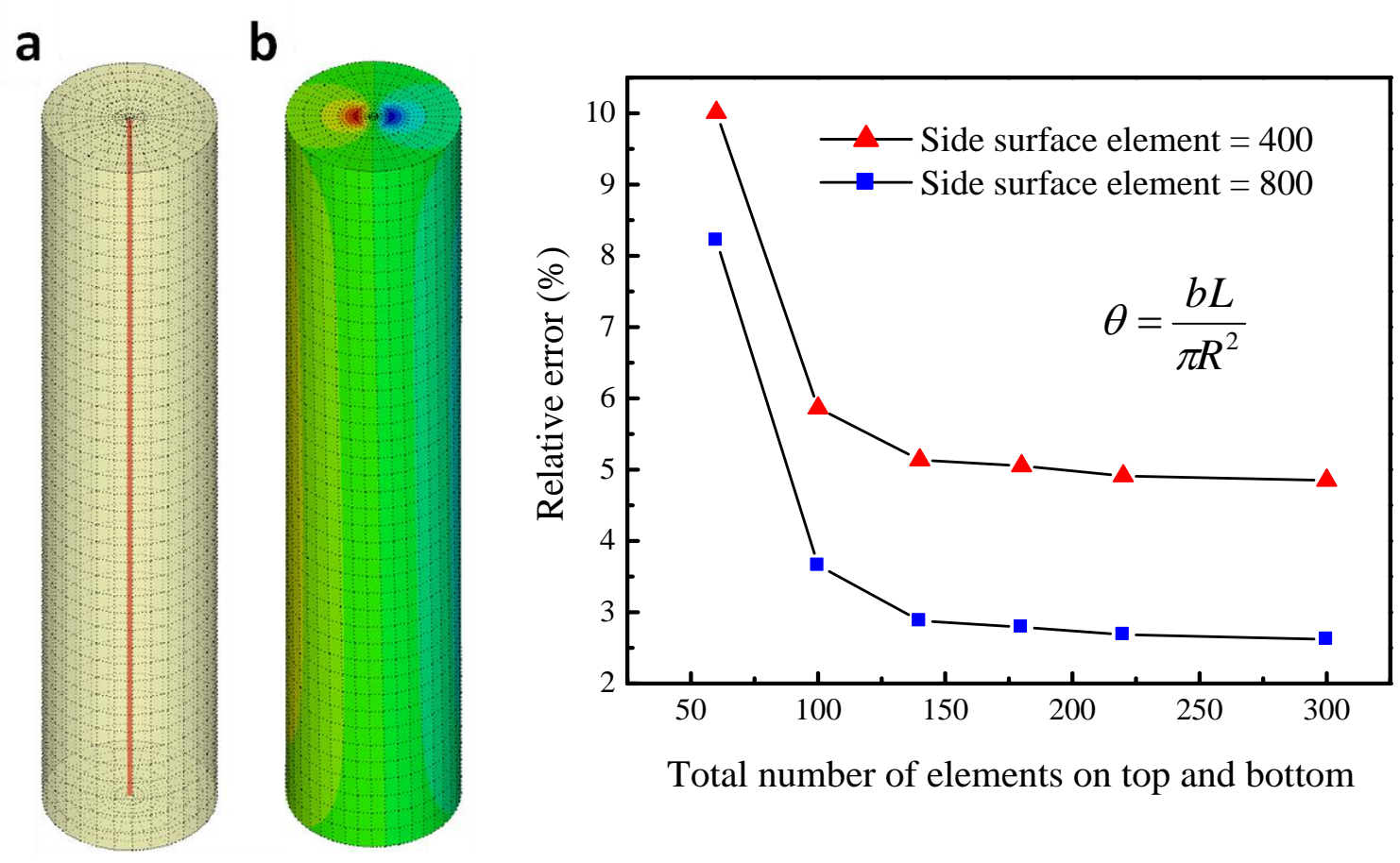

Total number of elements on top and bottom

Figure 3.3 Numerical results of Eshelby twist by a coaxial screw dislocation, (a) the configuration of a coaxial screw dislocation in a meshed cylinder, (b) the distributions of displacement and stress fields from numerical results, (c) relative error in the twist between two cross-sections of a cylinder, located at $5 \mathrm{R}$ and $6 \mathrm{R}$ from the bottom surface, respectively, for different numbers of surface elements (Displacement magnification: 100).

numbers of surface elements is shown in Figure 3.3. It is clear that relative error decreases with increasing the number of elements, either on the sides of the cylinder or on the top and bottom planes. Since the dislocation in analytical solution is infinitely long that is different from our model, the relative error only converges to $2 \%$ rather than approaches absolute zero.

\subsubsection{Image stress of a straight edge dislocation in a cylinder}

Consider comparison of the image stress of a straight edge dislocation inside a cylinder. As shown in Figure 3.4, the line direction of the edge dislocation lies in Z-axis (perpendicular to the paper) and the Burgers vector is along $\mathrm{X}$-axis. The analytical solution 


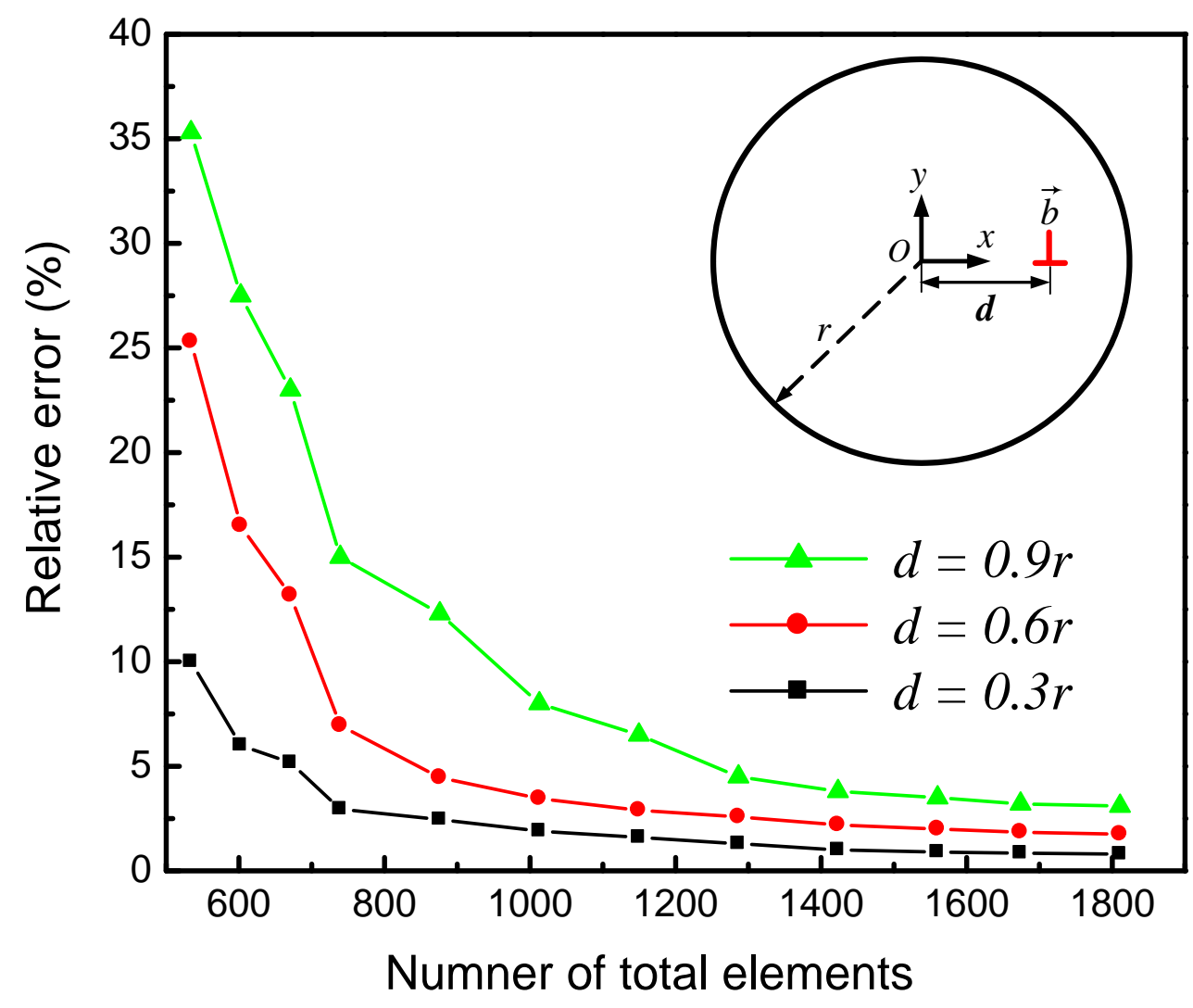

Figure 3.4 The relative error as a function of the number of surface elements on the cylinder for the image force on an edge dislocation located at $\mathrm{d}=0.3 \mathrm{r}, 0.6 \mathrm{r}$ and $0.9 \mathrm{r}$.

can be obtained by a complex image construction [16]. The stress component that gives rise to a PK force on the dislocation itself is $[7,16]$

$$
\sigma_{x y}^{i m g}=\frac{\mu b d}{2 \pi(1-v)\left(r^{2}-d^{2}\right)}
$$

where $\mathrm{d}$ is the distance offsetting from the origin along $\mathrm{X}$-axis and set to $0.3 \mathrm{r}, 0.6 \mathrm{r}$ and $0.9 \mathrm{r}$ in our test, the cylinder radius $\mathrm{r}=250 \mathrm{~nm}$ and height of cylinder $\mathrm{H}=1500 \mathrm{~nm}$. From eq. (3.33), we could see the edge dislocation is drawn towards the surface and so is the screw dislocation in Ref. [16]. As shown in Figure 3.4, the relative errors decay fast with the total number of elements increase. In addition, the numerical errors depend on the offsetting 
distance, i.e. numerical results converge faster for the dislocation near the center of cylinder than for dislocations near the cylinder surface. This phenomenon results from the feature of BEM. Since the elements of BEM mesh just located on the cylinder surface, the calculated stress fields on inside points far from the surface are more accurate than those on points near surface with a given number of elements [11]. When the number of meshed elements increases to a high level, the numerical image stress on dislocation close to surface still converges. However, numerical method itself is an approximation and could not calculated the image stress on the dislocation in an "infinitely long" cylinder as assumed in the ideal model [16], and hence only relative low rates of convergence can be arrived at by comparing with the reference analytic solution.

In addition, when dislocations intersect a free surface, we extended a virtual segment from the point of intersection with the free surface and continue tangent with the intersecting line to make the stress field computed correctly, which also has been validated by Weinberger et al [17].

\subsubsection{Image force on a screw dislocation in thin film}

The analytic solution of the image force on an infinitely long screw dislocation lying parallel to the free surfaces has been calculated by Hirth and Lothe by considering an infinite series of image dislocations originating from both sides of free surface[3].

The $\mathrm{z}$-component of the force $\mathrm{F}_{\mathrm{img}}$ acting on a screw dislocation at position 1 has the form

$$
\boldsymbol{F}_{i m g}=\frac{\mu b^{2}}{4 h} \cot \left(\frac{\pi l}{h}\right)
$$



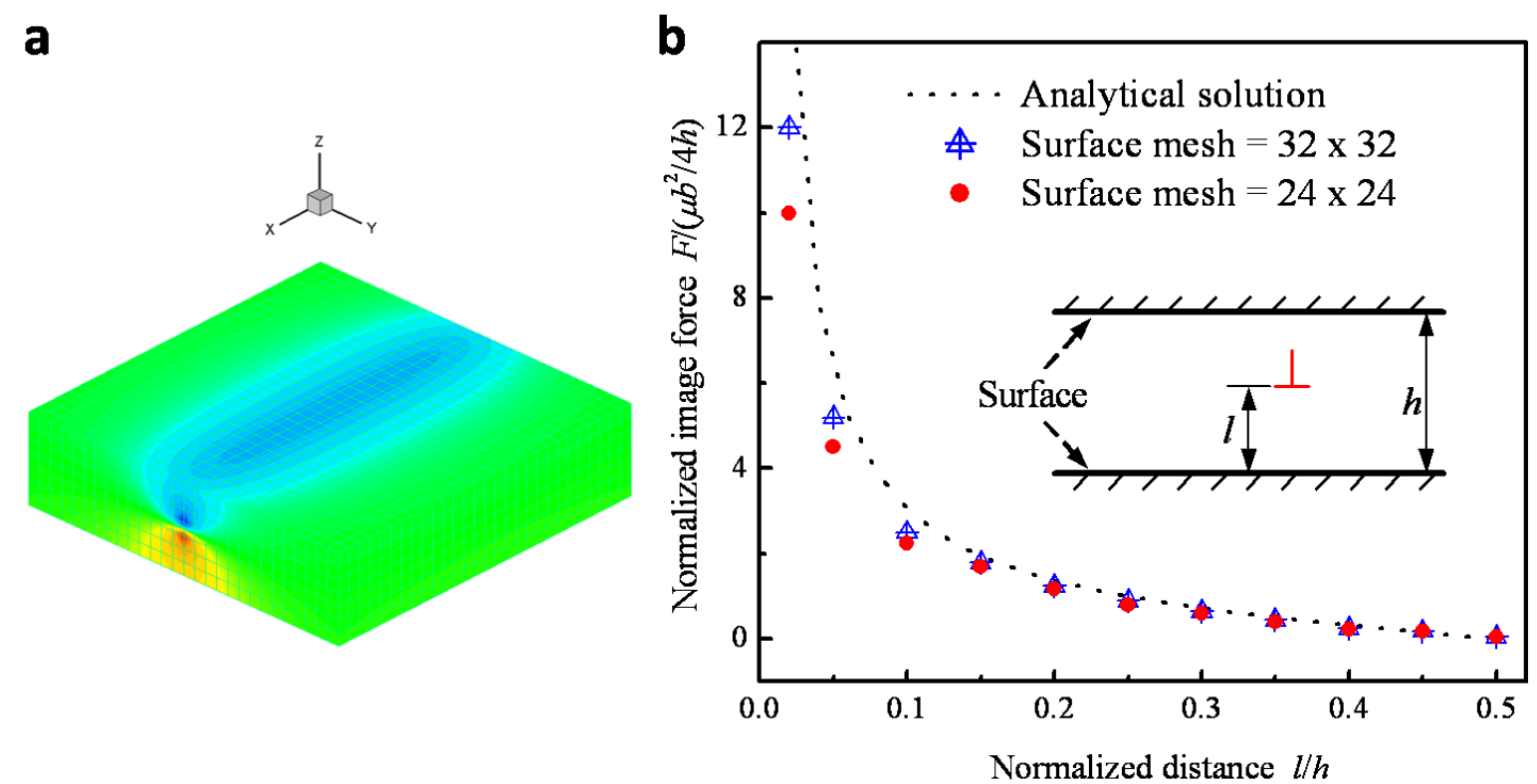

Figure 3.5 Numerical results of image force on long screw dislocation in thin film, (a) the configuration of a screw dislocation in a meshed film, (b) comparison of numerical results with analytic results.

where $\mathrm{h}$ is the film thickness and $\mu$ is the shear modulus. The simulation box used for the numerical calculation is shown in Figure 3.5. The film thickness and width are set to be 500 $\mathrm{nm}$ and $2000 \mathrm{~nm}$, respectively.

Figure 3.5 shows the comparison of numerical results with analytical results. The numerical values are all taken from the image force on the middle segment of this screw dislocation. We can see a refinement of surface mesh yields better results, especially when the dislocation moving the center of the film. However, we cannot generate infinitely long screw dislocation in our study, thus the difference between numerical and analytical results becomes lager when the dislocation approach surfaces. In a realistic case, thin films always have edges and our numerical method still can be used in the simulations on thin films.

3.2.5 Effect of image stresses on the flow stress 
In order to study the effects of image stresses on the flow stress, we generated a set of micropillars with the same diameter $\mathrm{D}=500 \mathrm{~nm}$ and height $\mathrm{H}=1500 \mathrm{~nm}$ with the aspect ratio equals to $\mathrm{H}: \mathrm{D}=3: 1$ with the same initial density $\rho=2.0 \times 10^{12} \mathrm{~m}^{-2}$. All dislocation sources were composed of FR sources randomly set on all twelve $<011>\{111\}$ slip systems with random lengths. Following the results in section 3.2.3, we chose the optimized 1560 elements as the default mesh size on all micropillars, which is more computationally efficient with negligible effect on the results compared with finer mesh sizes. And the experiment-like load procedure described in Chapter 4 was used in all simulations with the applied stain rate of $100 \mathrm{~s}^{-1}$.

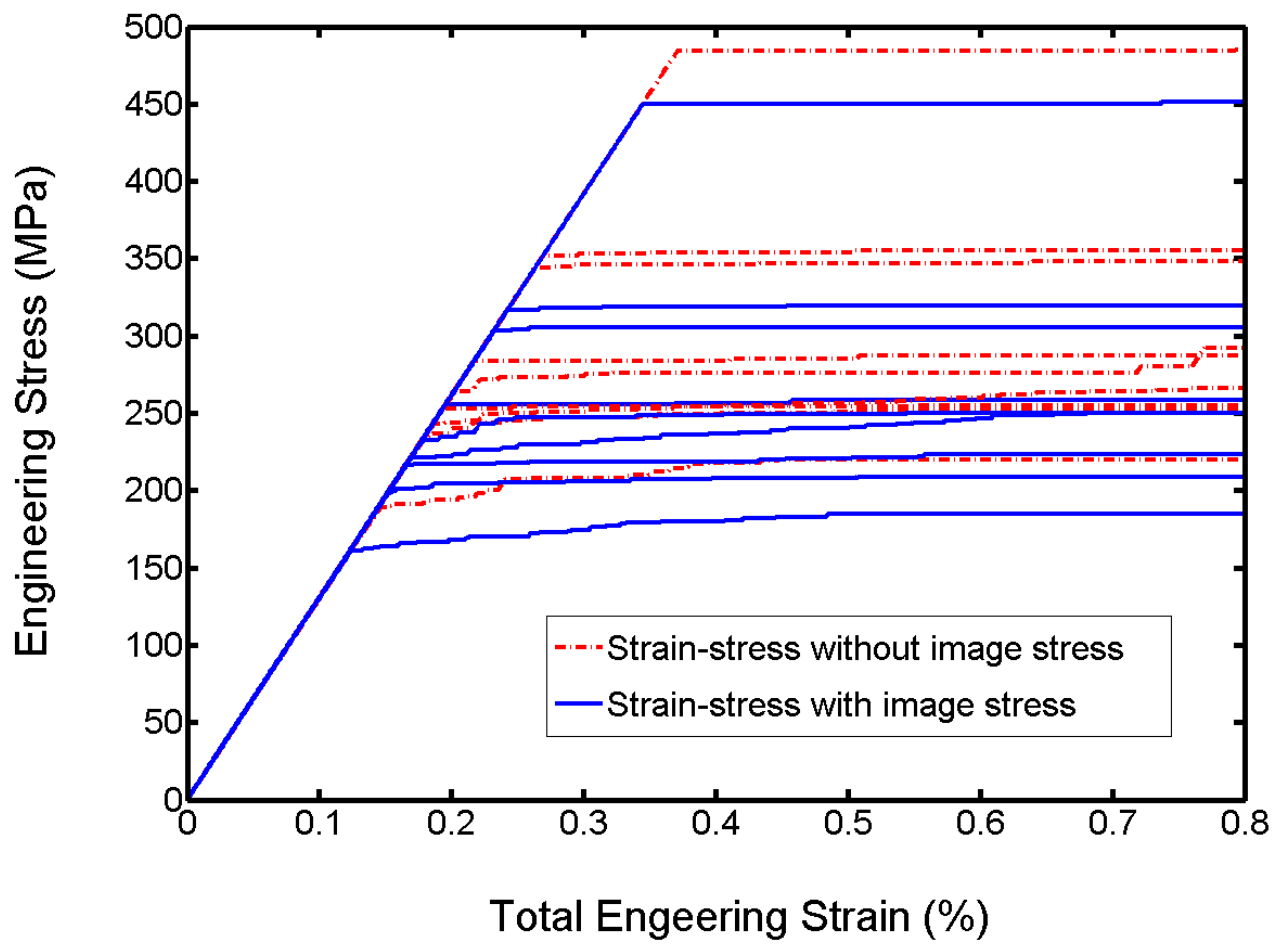

Figure 3.6 Comparison of flow stresses of the micropillars with and without image stresses 
As shown in Figure 3.6, it is clear that the flow stresses for samples with image stresses are lower than those ignoring image stresses, which results from the attractive image forces on internal dislocations from the free surface. As the dislocation sources start emitting dislocations, the image stresses assist their glide towards the surface, i.e. the image stresses assist the mobile sources in hastening the dislocation glide. This enhanced plastic flow produces a softening effect reflected by the stress-strain curves. The relative differences between flow stresses with and without considering image stresses vary from $10 \%$ to $20 \%$. The main factor affecting the relative difference is the distance between the activated dislocation sources and free surfaces. As we discussed in previous section, the magnitude of image stress increases with the decrease of the distance between the dislocation source and free surfaces, so when the activated dislocation located near free surfaces, the influence from image stresses will be stronger and cause larger relative differences on flow stresses. Consequently, the effect of image stresses decrease fast with the increase of sample sizes [10], since lager samples have lower surface to volume ratios and more dislocation sources close to the center of samples at a given dislocation density. 


\section{References}

[1] Vandergiessen E, Needleman A. Discrete dislocation plasticity - a simple planar model. Modelling and Simulation in Materials Science and Engineering 1995;3:689.

[2] Fivel MC, Gosling TJ, Canova GR. Implementing image stresses in a 3D dislocation simulation. Modelling and Simulation in Materials Science and Engineering 1996;4:581.

[3] Hartmaier A, Fivel MC, Canova GR, Gumbsch P. Image stresses in a free-standing thin film. Modelling and Simulation in Materials Science and Engineering 1999;7:781.

[4] Lemarchand C, Devincre B, Kubin LP. Homogenization method for a discrete-continuum simulation of dislocation dynamics. Journal of the Mechanics and Physics of Solids 2001;49:1969.

[5] Khraishi TA, Zbib HM. Free-surface effects in 3D dislocation dynamics: Formulation and modeling. Journal of Engineering Materials and Technology-Transactions of the Asme 2002;124:342.

[6] Tang MJ, Cai W, Xu GS, Bulatov VV. A hybrid method for computing forces on curved dislocations intersecting free surfaces in three-dimensional dislocation dynamics. Modelling and Simulation in Materials Science and Engineering 2006;14:1139.

[7] Weinberger CR, Cai W. Computing image stress in an elastic cylinder. Journal of the Mechanics and Physics of Solids 2007;55:2027.

[8] Weinberger CR, Aubry S, Lee SW, Nix WD, Cai W. Modelling dislocations in a free-standing thin film. Modelling and Simulation in Materials Science and Engineering 2009;17.

[9] Weygand D, Friedman LH, Van der Giessen E, Needleman A. Aspects of boundary-value problem solutions with three-dimensional dislocation dynamics. Modelling and Simulation in Materials Science and Engineering 2002;10:437.

[10] El-Awady JA, Biner SB, Ghoniem NM. A self-consistent boundary element, parametric dislocation dynamics formulation of plastic flow in finite volumes. Journal of the Mechanics and Physics of Solids 2008;56:2019.

[11] Becker A. The Boundary Element Method in Engineering: A Complete Course McGraw-Hill International, 1992. 
[12] Aliabadi MH. The Boundary Element Method : Applications in Solids and Structures Wiley 2002.

[13] Liu XH, Schwarz KW. Modelling of dislocations intersecting a free surface. Modelling and Simulation in Materials Science and Engineering 2005;13:1233.

[14] Hull D, Bacon DJ. Introduction to Dislocations, 4 edition. Butterworth-Heinemann, 2001.

[15] Eshelby JD. Screw dislocations in thin rods. Journal of Applied Physics 1953;24:176.

[16] Eshelby JD. Chapter 3 Boundary problems. In: Nabarro FRN, editor. Dislocations in Solids, vol. Volume 1. North-Holland Publishing Co 1979. p.167.

[17] Weinberger CR, S. Aubry, Lee SW, Cai W. Dislocation dynamics simulations in a cylinder. IOP Conf. Series: Materials Science and Engineering 2009;3:7. 


\section{CHAPTER 4}

\section{SIZE EFFECTS ON PLASTICITY OF FCC SINGLE CRYSTALS}

The goal of this work was to model the experiment as closely as possible. In addition to creating initial conditions that best mimic experiment, the simulations discussed here also include two effects not generally included in previous simulations: surface forces and cross-slip. Surface forces were included through the use of the boundary-element method. Cross slip was modeled with a stochastic method and was found to play a critical role in dislocation behavior. Finally, the effects of loading direction were also studied.

\subsection{Simulation procedures}

The 3D DD SIMULATIONS framework described in Chapter 2 has been used to simulate the mechanical behavior of $\mathrm{Ni}$ single crystals under uniform compression. . For the simulations in this work, the materials properties of nickel are used: shear modulus $\mu=76$ GPa, Poisson's ratio $v=0.31$, and lattice constant $\mathrm{a}=0.35 \mathrm{~nm}$. The dislocation mobility is taken to be $10^{-4} \mathrm{~Pa}^{-1} \mathrm{~s}^{-1}$ in the calculations [1]. In finite volume problems, it is necessary to include both the solution for dislocations in an infinite medium and the complementary elastic solution that satisfies equilibrium at external and internal boundaries. To evaluate image fields, the boundary element method (BEM) has been introduced into our dislocation dynamics simulations and performed as follows. First, the elastic stress field in an infinite medium resulting from all dislocations is evaluated. Then tractions at the surfaces of the finite crystal owing to the dislocation stress field are determined, reversed and placed on the surface as traction boundary conditions. These traction boundary conditions, as well as any other imposed constraints, are employed in BEM to calculate all unknown surface tractions 
and displacements. Finally, the image stress field is calculated and the result is superimposed as indicated in Chapter 3.

Cross slip, in which screw dislocations leave their habit planes and propagate to another glide plane [2-3], plays a key role in macroscopic plastic deformation of FCC materials. However, questions of how cross slip operates and its importance at the micron and submicron scales are still under debate. In this study, we adopt a sophisticated cross-slip model developed by Kubin and co-workers [4-5] that is based on the Friedel-Escaig mechanism of thermally-activated cross-slip [6-7]. In this model, the probability of cross-slip of a screw segment with length $L$ in the discrete time step is determined by an activation energy $V_{\text {act }}\left(|\tau|-\tau_{I I I}\right)$ and the resolved shear stress on the cross-slip plane $\tau$,

$$
P=\beta \frac{L}{L_{0}} \frac{\delta t}{\delta t_{0}} \exp \left[-\frac{V_{a c t}}{k T}\left(|\tau|-\tau_{I I I}\right)\right]
$$

where $\beta$ is a normalization constant, $k$ is the Boltzmann constant, $T$ is set to room temperature, $V_{a c t}$ is the activation volume, and $\tau_{I I I}$ is the stress at which stage-three hardening starts. In nickel, $V_{a c t}$ is equal to $420 b^{3}$ with $b$ the magnitude of Burgers vector [8], $\tau_{I I I}=55 \mathrm{MPa}$ [9], and $L_{0}=1 \mu \mathrm{m}$ and $\delta t_{0}=1 \mathrm{~s}$ are reference values for the length of the cross-slipping segment and for the time step. Eq. (4.1) describes the thermal activation of cross-slip, expressed in terms of a probability function. A stochastic (Monte Carlo) method is used to determine if cross slip is activated for a screw dislocation segment. At each time step, the probabilities for cross slip of all screw segments are calculated using equation (3). For each screw segment, the probability $P$ is compared with a randomly generated number $N$ between 0 and 1 . If the calculated $P$ is larger than $N$, cross slip is activated, otherwise, the cross slip is disregarded [1, 10]. 
Our goal is to mimic the experimental conditions as well as possible. To that end, we start by creating a "bulk" sample, from which we will "cut" a set of cylindrical samples. To model the bulk, we assume a cubic cell with periodic boundary conditions and a size $3 \times 3 \times 3$ $\mu \mathrm{m}^{3}$ containing a set of FR sources with an initial density equal to $2.0 \times 10^{12} \mathrm{~m}^{-2}$. The FR sources (straight dislocation segments pinned at both ends) were randomly set on all twelve $<011>\{111\}$ slip systems with random lengths as shown in Figure 4.1a. After compression in
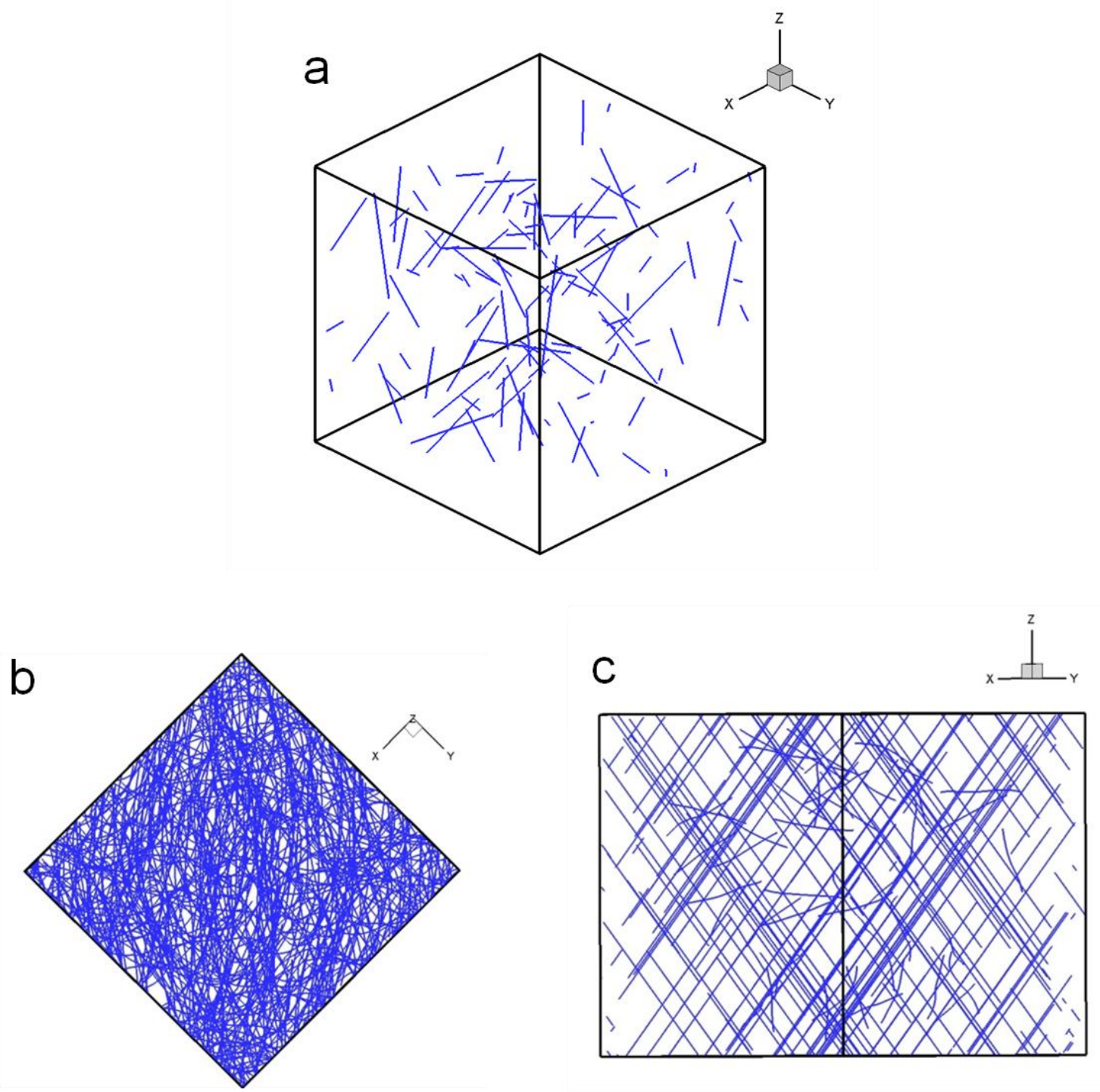

Figure 4.1 Dislocation structures in $3 \times 3 \times 3 \mu \mathrm{m}^{3}$ cube sample. (a) Initial dislocation structure in [111] view, (b) deformed structure in [001] view, (c) deformed structure in [110] view. 
the [111] direction to a plastic strain of $0.1 \%$, the distribution of dislocations evolves to the structure shown Figure4.1b with a dislocation density of about $2.5 \times 10^{13} \mathrm{~m}^{-2}$. The cubic sample was unloaded (i.e., relaxed) and cylinders of various sizes (representing micropillars) were cut out of the bulk sample. The diameters $D$ of the micropillars were $D=1.0,0.75$ and $0.5 \mu \mathrm{m}$, and the aspect ratio was set to $D: H=1: 2$, where $H$ denotes the height of micropillars. Subsequently, the deformed dislocation microstructrures were relaxed only under the influence of image and interaction forces as shown in Figure 4.2a and b. Most of the micropillars were cut along the [001] direction, except for three samples along the [269] direction with $D=1.0 \mu \mathrm{m}$. This procedure delivers what we assume to be realistic initial dislocation structures that include internal FR sources of different sizes, single-ended sources (spiral sources with one end pinned inside the cell and the other at the surface), surface
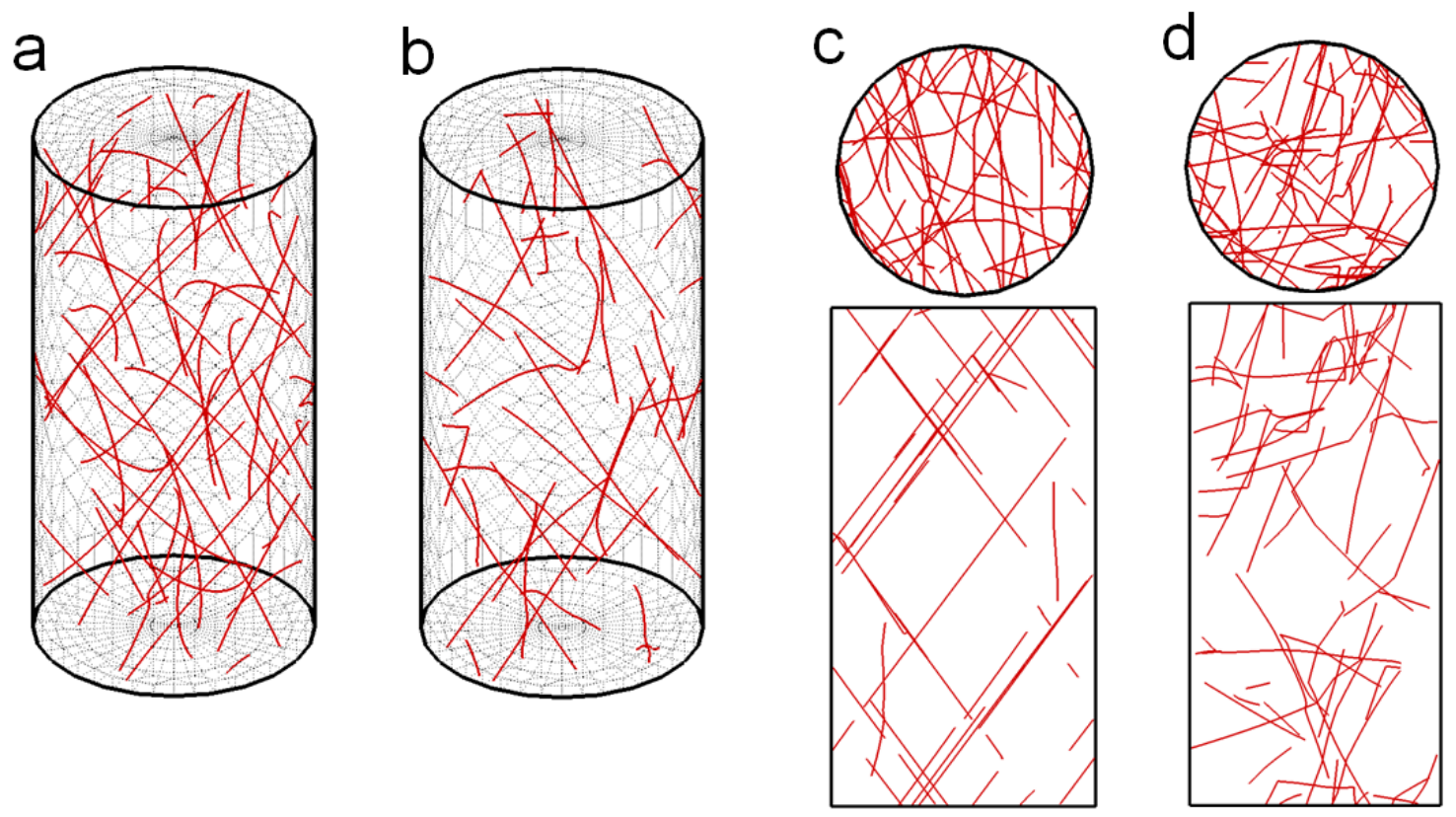

Figure 4.2 Dislocation structures in cut samples with $D=1.0 \mu \mathrm{m}$ (Dotted lines are BEM meshes). (a) Cutting from [001] before relaxation with $\rho=2.7 \times 10^{13} \mathrm{~m}^{-2}$ ([111] view), (b) cutting from [001] after relaxation with $\rho=1.9 \times 10^{13} \mathrm{~m}^{-2}$ ([111] view), (c) cutting from [001] direction with $\rho=$ $1.9 \times 10^{13} \mathrm{~m}^{-2}$ (upper [001] view), (lower [110] view), (d) cutting from [269] direction with $\rho=$ $2.0 \times 10^{13} \mathrm{~m}^{-2}$ (upper [001] view), (lower [110] view). 
dislocations (both ends at surface) and dislocation reactions, such as junctions. The dislocation densities after relaxation were all in the range of 1.0 to $2.0 \times 10^{13} \mathrm{~m}^{-2}$ and were consistent with conditions observed in experiments [11].

We simulated the experimental loading conditions of Dimiduk and coworkers [11-15] in our computations, in which a mixture of constant displacement rate and creep-like loading conditions were employed; the applied stress was discretely increased by a small fixed value $(\delta \sigma)$ every time the plastic strain rate approached zero. When the plastic strain rate was smaller than the applied rate, the applied load was increased by $2 \mathrm{MPa}$, i.e. $\delta \sigma=2 \mathrm{MPa}$, for $\dot{\varepsilon}^{p}<\dot{\varepsilon}$, while the applied stress was kept constant when the plastic strain rate was equal to or higher than that of the applied rate, i.e. $\delta \sigma=0$, for $\dot{\varepsilon}^{p}>\dot{\varepsilon}$.

In all simulations, compression loading in [001] direction was performed under a constant strain rate of $200 \mathrm{~s}^{-1}$. To identify the effects of strain rate, several simulations were performed with strain rates as low as $50 \mathrm{~s}^{-1}$. The results from those simulations did not show any significant difference from those seen at $200 \mathrm{~s}^{-1}$. We found that a strain rate of $200 \mathrm{~s}^{-1}$ is computationally efficient with negligible effect on the results while also being lower than the strain rates used in other similar simulations [16-19].

To investigate the effects of loading direction, as well as to make a direct comparison with the experimental results of Dimiduk et al. [13], we also prepared three $1.0 \mu \mathrm{m}$ samples oriented in the [269] direction. We see distinct differences in the two typical initial dislocation structures from the [001] and [269] samples as shown in Figure 4.2c and d, respectively. Since the stress was then applied along the [001] axis, the simulations correspond to a single-slip direction for samples cut from the [269] direction and along a multi-slip direction for samples cut along the [001] direction. For the single-slip case, only the $\frac{1}{2}[101](\overline{1} 11)[20]$ systems are active, each with the same Schmid factor of 0.41 , whereas the other four slip systems have zero Schmid factors and are inactive. 


\subsection{Effect of loading direction}

The stress-strain behavior for all simulations based on $1.0 \mu \mathrm{m}$ samples is shown in Figure 4.3a, while the equivalent experimental results for single-crystal nickel are shown in Figure 4.3b. Comparing Figure 4.3a and Figure 4.3b, we see that the flow stress of the multi-slip simulations (from the [100] samples) and the single-slip simulations (from the [269] samples) are both similar to each other and agree well with the experimental results, which employed loading along the [269] single-slip direction. In our simulations, only one, or at most a few, mobile dislocations determined the strength at small volumes. Thus, multiple-slip simulations and single-slip simulations exhibited similar results. The agreement between the results for single-slip and multi-slip loading is not surprising in light of recent results. Norfleet et al. [11] recently examined cut foils from deformed pillars and found that for samples $<20 \mu \mathrm{m}$ in diameter, multiple slip systems are always active regardless of the loading direction. In addition, a recent theoretical study by $\mathrm{Ng}$ et al. concluded that Schmid's law, which states that plastic flow will occur on the slip system with the largest Schmid factor, no longer holds for microcrystal deformation, because of the increase of the probability to activate sources with low Schmid factors in small samples, as the overall number of dislocation sources decreases with the sample diameter [21]. Thus, both experiment and modeling indicate that single-slip and multiple-slip deformation should be similar in these small samples.

Recent 3D DD simulations that were based on an initial dislocation structure within the cylinder consisting of only internal FR sources showed linear elastic loading up to the yield point $[17-19,22]$. In contrast to those results, our simulations showed a large amount of "microplasticity" at low loads (shown in Figure 4.3a), in agreement with the experimental results (shown in Figure 4.3b). This early-stage plasticity is often the result of essentially free dislocations being driven out of the system. These dislocations could either be weakly 

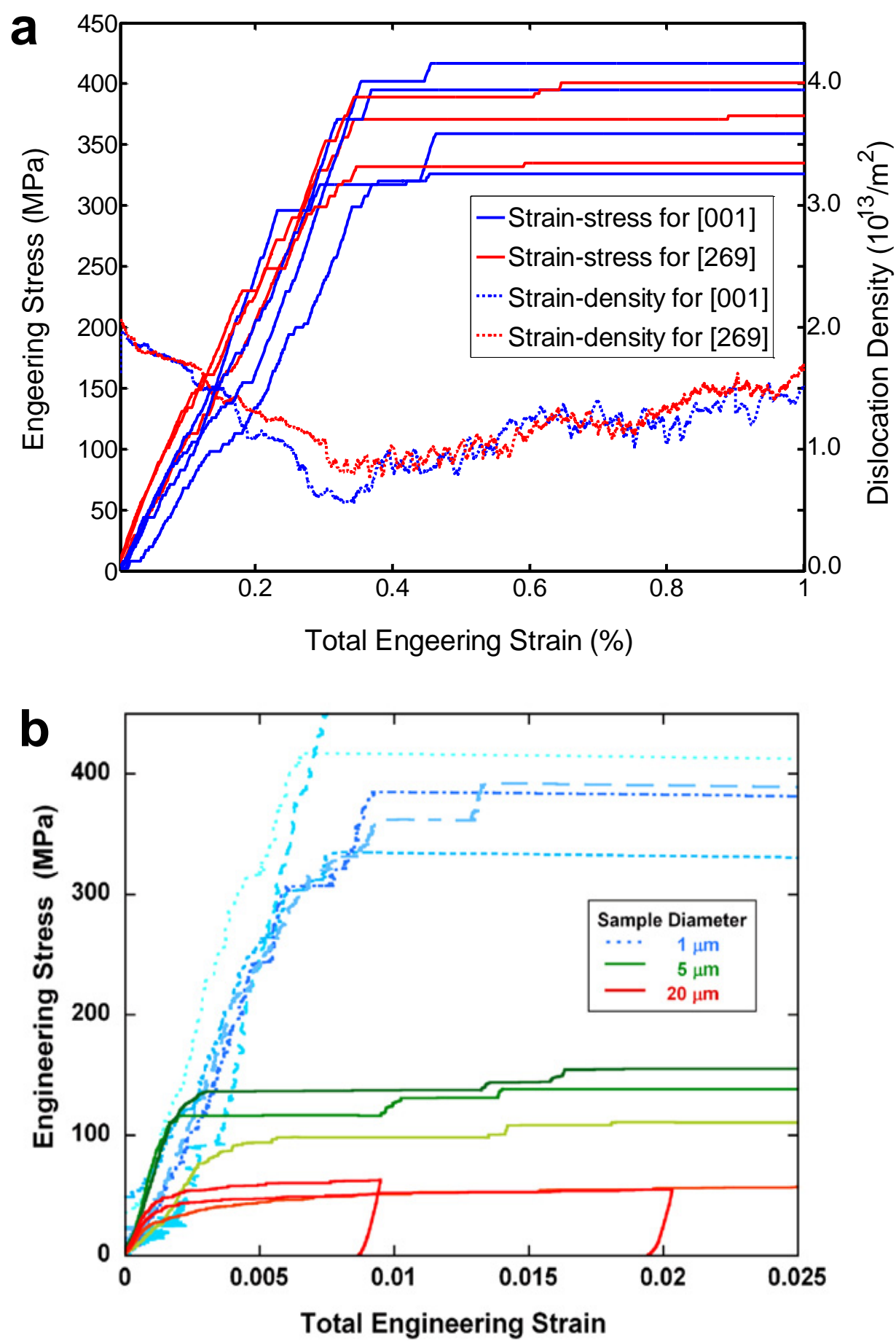

Figure 4.3 Comparison of stress-strain curves of simulation and experiment. (a) Stress-strain and typical density-strain curves obtained from simulation with $D=1.0 \mu \mathrm{m}$, (b) Stress-strain curves obtained from experiment [13]. 
entangled or pre-existing at the surface. The movie A in supplementary materials of ref. [23] illustrates one example of how a dislocation junction unzipped and then was driven out of the sample as load was increased. The presence of such dislocations can be explained as follows. After cutting the cylinders out of the bulk system, we relax the dislocation positions. While some of surface dislocations escape to the surface owing to the large image forces, many dislocations can be trapped by dislocation reactions, such as junctions, or be near the center of the sample where the image forces are insufficient to cause any significant movement [24]. In experiments, a large number of surface dislocations of different sizes might also exist in the micropillars. These dislocations may be generated by the act of the cutting, but could also arise from defects caused by preparation procedures themselves, such as focused ion beam milling [25].

As the loading is increased, the motion of free dislocations is gradually activated. The dislocations then sweep quickly across the slip plane, exiting the micropillar, leading to a rapid reduction in dislocation content referred to as "dislocation starvation." The easy movement of these free dislocations leads to a plastic strain rate that approaches the applied strain rate, which causes the applied stress increment to approach zero, as mentioned in the discussion of the loading scheme. Thus, we see an initial small strain burst on the stress-strain curves. The amount of plastic strain in our simulations is smaller than that observed in experiments, which likely arises from two possibilities. Experimental samples are all processed by focused ion beam milling, leading to many surface defects that can generate plastic strain under loading [26]. Also, the $200 \mathrm{~s}^{-1}$ strain rate in our simulation is four orders of magnitude larger than those in the experiments, which have a creep-like loading and thus can carry more deformation at low loads.

Owing to the escape of free dislocations, the dislocation density in all samples will decrease in the early stages as reflected by the density-strain curves in Figure 4.3a. In previous 3D dislocations dynamics simulations [17-19, 22], only permanent internal FR 
sources were used as the initial configuration. Thus, the dislocation density could not decrease even with the intermittent presence of mobile density-starved states. In our simulation procedure, in small pillars, only a few surface dislocations, a few jogged dislocations and no internal pinned points could be found in inside the cylinder. Under the combination of high image forces and increased applied loading (and no cross slip, as discussed below), all pre-existing dislocations can be quickly driven out of the pillar, which supports the "dislocation starvation" model in small samples. Recently, Shan et al. [27] directly observed that pre-existing dislocations could be driven out of the pillar with the entire length of the pillar being left almost dislocation free for pillars with diameter less than 130nm. This phenomenon, which was called "mechanical annealing," directly supports the ideas behind the "dislocation starvation" model in smaller samples. However, for pillars larger than $300 \mathrm{~nm}$, pre-existing dislocations could not be completely driven from the cylinder, which indicates that permanent pinning points exist in those micropillars and that the dislocation density will eventually increase following the initial "mechanical annealing". These experimental results agree well with what is observed in our simulations as plotted in Figure 4.3a. The dislocation density increase following "mechanical annealing" was caused by the activation of dislocation sources and dislocation multiplication with the increasing load arising from cross slip, as is described in next section.

\subsection{Cross-slip}

To investigate the influence of cross-slip on the mechanical response and evolution of the dislocation microstructure, an additional sample with $D=1.0 \mu \mathrm{m}$ was cut from the undeformed cube shown in Figure 4.1a. Thus, only Frank-Read and spiral sources were initially present, with an initial density of $1.8 \times 10^{12} \mathrm{~m}^{-2}$ in the sample. This sample was then put under load both with and without cross-slip enabled. In Figure 4.4 we show the comparison of microstructures and the stress and density evolution for these two cases. It is 
clear that the sample with cross-slip is softer than that without cross-slip, likely because cross-slip leads to more sources and thus greatly increased dislocation density, as shown in Figure 4.4d. We note that the cross-slip started at the onset of plastic flow.
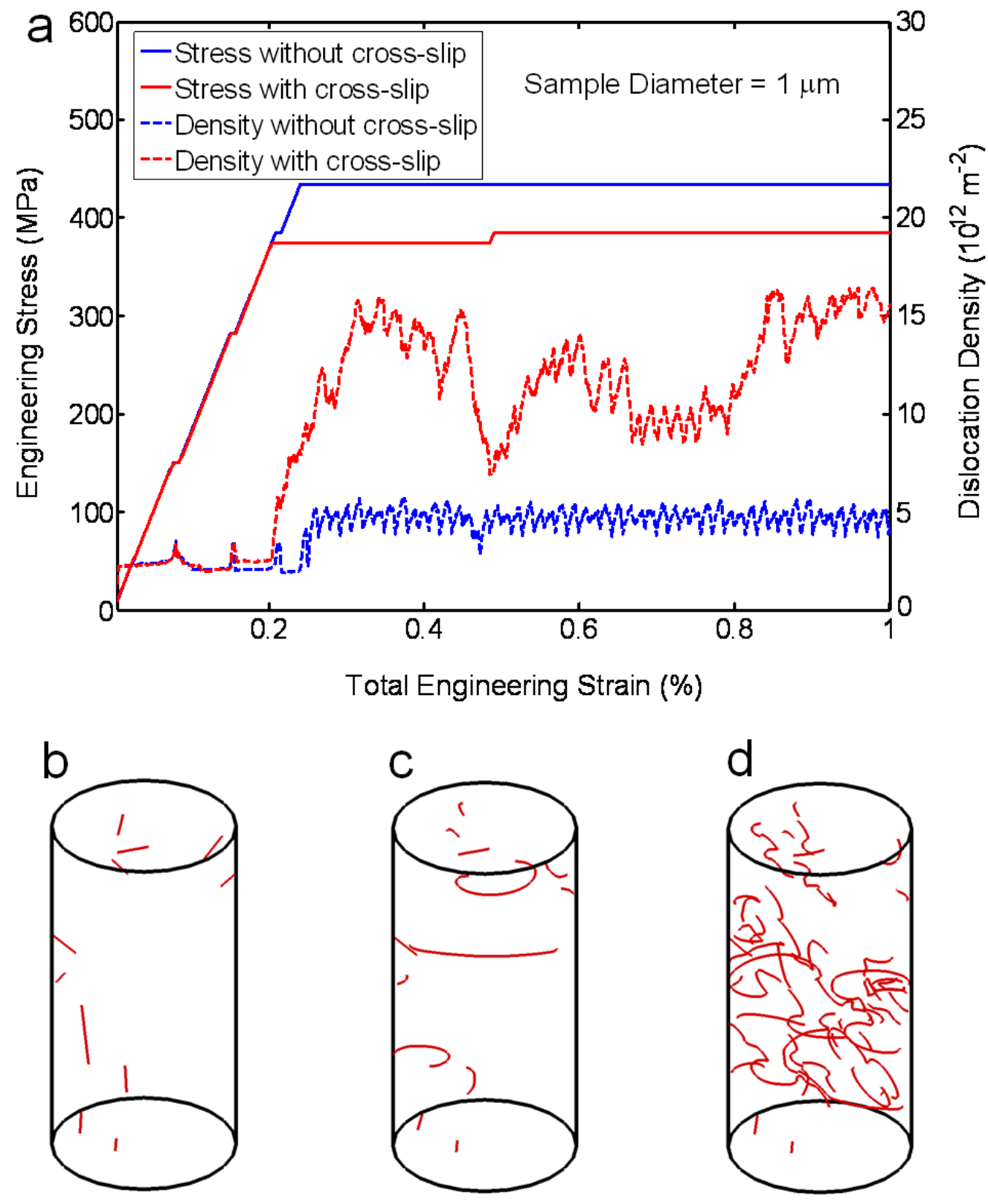

Figure 4.4 Comparison of the stress and density evolution with and without cross-slip. (a) stress and density curves, (b) initial dislocation structure, (c) dislocation structure without cross-slip at $1 \%$ strain and (d) dislocation structure with cross-slip at $1 \%$ strain. 

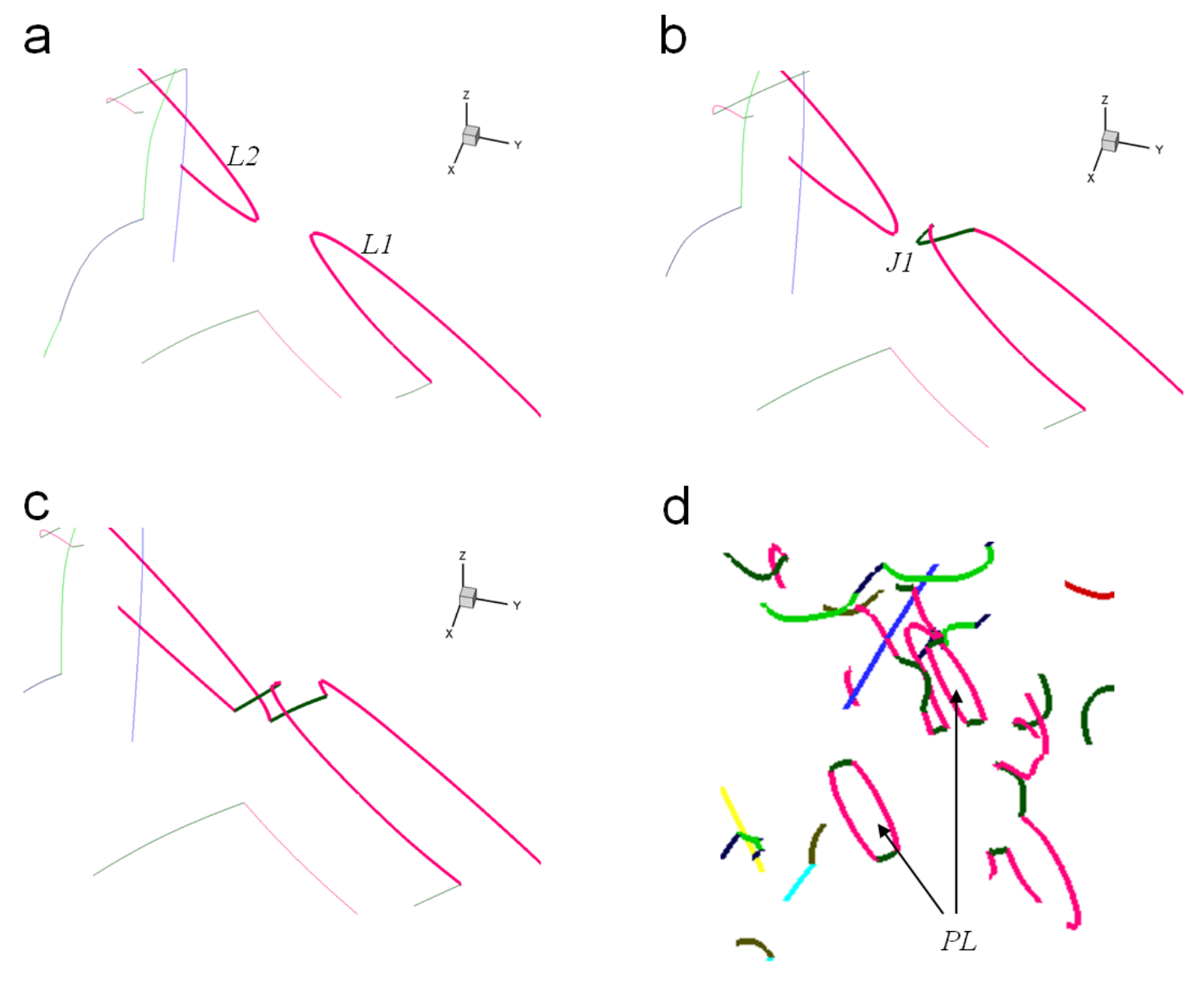

Figure 4.5 Plot of cross-slip on parallel dislocations and formation of prismatic loop (PL): pink line with $1 / 2[101](\overline{11} 1)$ and forest green line with $1 / 2[101](\overline{1} 11)$ : (a) two parallel dislocations slip on its own planes, (b) one dislocation cross-slip under the attractive force, (c) collinear reaction of the leading segments forming two superjogs, (d) prismatic loops.

Figure 4.5 shows a series of snapshots that illustrate how cross-slip activates secondary slip systems and enables oppositely signed screw dislocations on different planes to annihilate each other. The two red dislocations $L 1$ and $L 2$ have the same slip system $\frac{1}{2}[101](\overline{1} 11)$ on parallel glide planes but opposite initial orientations. Hence, there is an attractive force between the two dislocations that makes the screw segment $J l$ of dislocation 


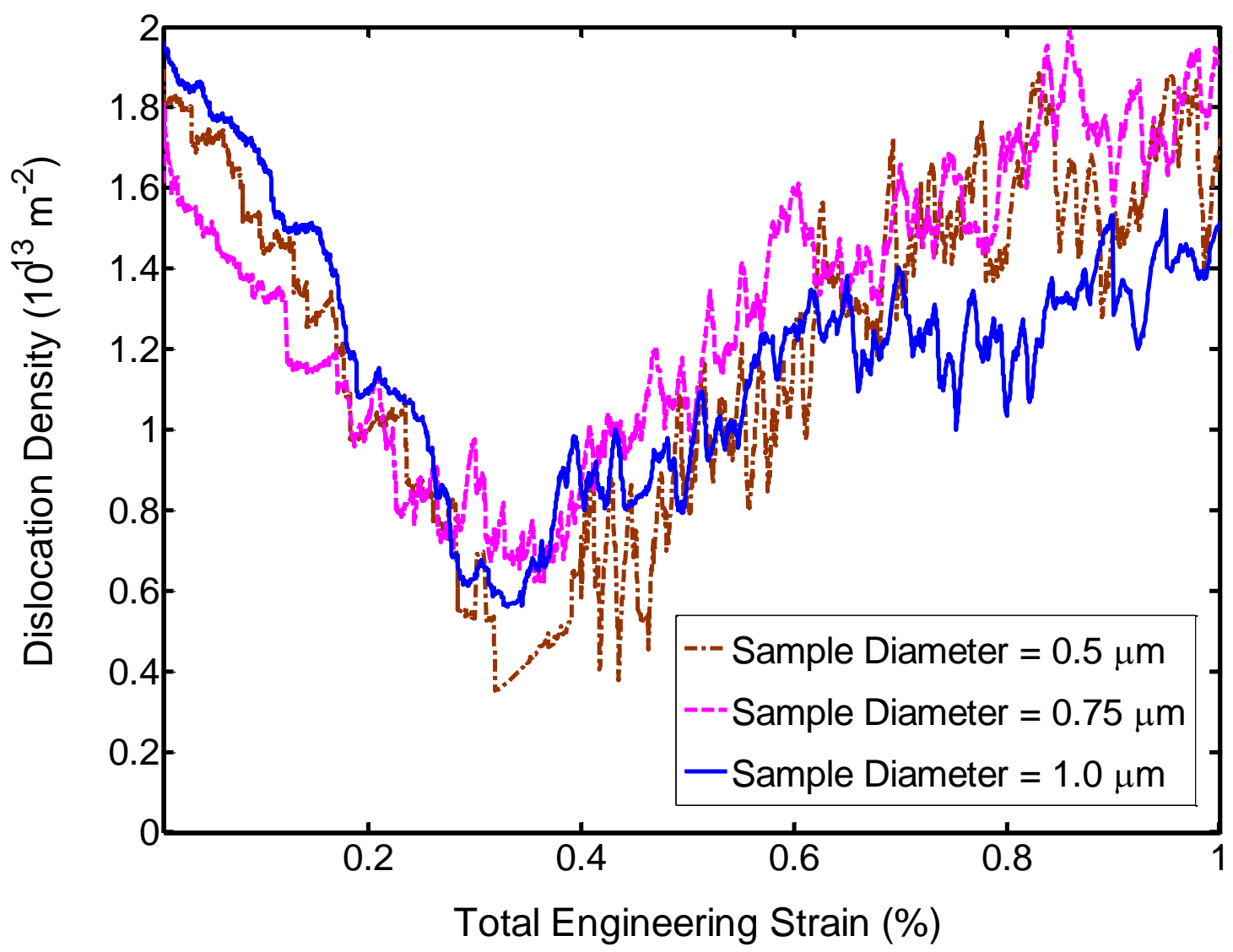

Figure 4.6 Evolution of dislocation density with total strain.

$L 1$ cross-slip on the plane $(\overline{1} \overline{1} 1) . J 1$ continues bowing out under the attractive force until its leading segments undergo a collinear reaction with the original dislocation L2 (they have the same Burgers vector and opposite line orientation). In Figure $4.5 \mathrm{c}$, we can see that two superjogs were left after the collinear reaction. Under the external stress field, the two arms of superjogs moved on their slip planes and formed a prismatic loop, as shown in Figure 4.5d. The prismatic loops are quite stable and can move only along the cylinder axis. Since this motion is difficult, the prismatic loops are fixed at the location at which the cross-slip occurred. They can then trap mobile dislocations, forming a dislocation forest as shown in Figure 4.5d, which has a strong influence on the subsequent plastic flow in small volumes. 
In Figure 4.6 we show the variation of dislocation density as a function of sample size and total strain. For all sizes studied in this study, the dislocation density initially dropped ("mechanical annealing"), followed by a steady increase (hardening). The dislocation density is reasonably insensitive to system size, with the point at which the density begins to rise occurring at approximately the same strain (approximately $0.4 \%$ ) for all samples. Below we shall discuss the behavior of the dislocation density in more detail.

The basic behavior of the hardening arises from the cross-slip mechanisms shown in Figure 4.7. At the beginning of the deformation, only a few dislocation sources are available after most of the free dislocations were driven out of the sample, as shown in Figure 4.7a and described above. Under increasing load, a spiral source $K l$ with Burgers vector $\frac{1}{2}[\overline{1} 01]$ was activated and moved in its slip plane (111) in Figure 4.7b. Screw segment $C 1$ then cross slipped on the slip plane (111) with the same Burgers vector, forming two joint corners $p 1$ and $p 2$, both of which then moved along the intersection line between the original slip plane and the cross-slip plane (Figure 4.7c, discussed in detail hereinafter). After extending on the slip plane under load, the original source $K 1$ was truncated by the free surface and then stopped moving in Figure 4.7d. However, the cross-slipped part C1 and non-cross-slipped parts $K 2$ and $K 3$ truncated from $K 1$ propagated smoothly until they encountered the free surface. In Figure 4.7e, the screw part $C 2$ on $C 1$ cross-slipped back to the original slip plane (111) (double cross-slip), a mechanism that generates considerable plastic strain in the deformation of bulk materials. Meanwhile, $K 2$ and $K 3$ behaved similarly to Frank-Read sources in the bulk, in that they annihilated each other and generated new dislocations $K 4$ and K5.

The major difference between multiplication processes observed in small volumes and those in the bulk is that the new dislocation, such as $K 5$, escape to the surface under the influence of image forces. In small volumes, it appears that the surface always confines 

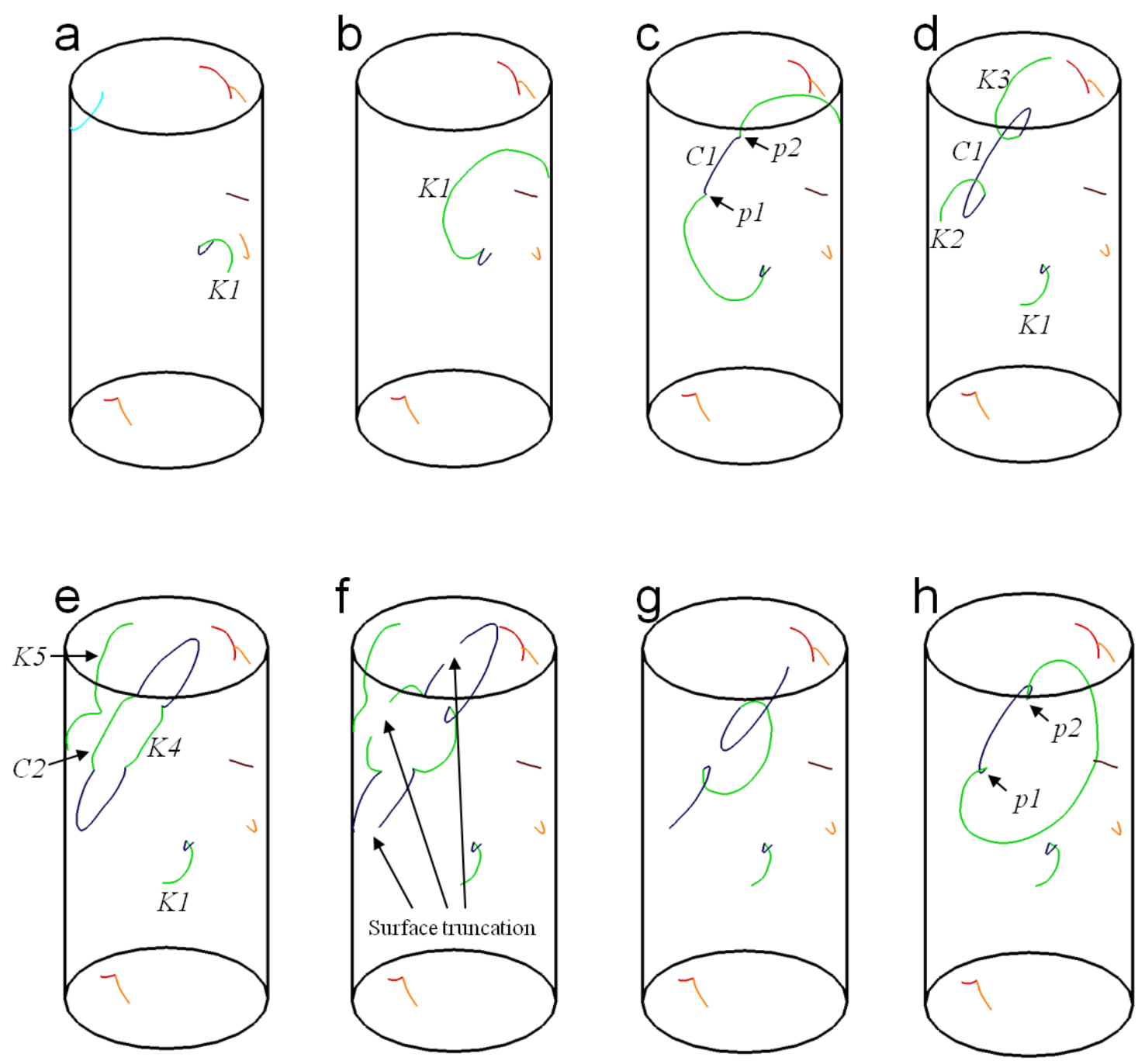

Figure 4.7 Plot of cross-slip forming dynamic FR source: green line with 1/2[1]01](111) and navy line with $1 / 2[\overline{1} 01](1 \overline{1} 1)$, see details in text.

dislocation propagation, having a potent hardening effect as sample size decreases because of the shortening of the dislocation sources. In our simulations, this "source-truncation" [28] effect is reflected in Figure 4.7d, in which the original spiral source $K l$ was pinned after being truncated by the surface. From Figure $4.7 \mathrm{e}$ to $\mathrm{h}$, the two joint corners $p 1$ and $p 2$ formed a new dynamic FR source that continuously generated dislocations on two different slip planes, leading to the constant-stress avalanches reflected on the stress-strain curves. 
However, this dynamic FR source is not as stable as regular FR sources having permanent pinning points, since the two endpoints of a dynamic FR source might move out of the sample surface, thereby releasing the dynamic source. The stability of these sources increases with the increased sample size, affecting their contribution to the accumulated plastic strain of the sample and the increase of dislocation density.

\subsection{Exhaustion hardening}

In our simulations, superjogs and dynamic spiral sources, as illustrated in Figure 4.8a and b, were always formed by cross-slip or collinear reactions [29] combined with the truncation by free surfaces. The superjog $\mathrm{AO}_{1} \mathrm{O}_{2} \mathrm{~B}$ with two ends $A$ and $B$ at the surface in Figure 4.8a is similar to jogs artificially generated in Ref. [30], except that in our simulations they were formed naturally. One difference in behavior between [30] and the present results is that the middle segment $O_{1} O_{2}$ bowed out under sufficient force in this study. Under loading, the two dislocation arms, $A O_{1}$ and $\mathrm{BO}_{2}$ operated independently around the jog corners $O_{1}$ and $O_{2}$, producing continuous plastic flow. When $O_{1} O_{2}$ is short enough, the superjog $A O_{1} O_{2} B$ formed an intermediate jog, as the dislocations arms $A O_{1}$ and $B O_{2}$ interacted like dislocation dipoles and could not pass by one another except at a high stress [31]. Once the resolved shear stress on segment $O_{1} O_{2}$ is large enough, it bowed out like an FR source. If it was truncated by the free surface, this superjog $A O_{1} O_{2} B$ transformed into two dynamic spiral sources, e.g., $A O B$ in Figure $4.8 \mathrm{~b}$. These two dislocation arms of these dynamic sources were rotated around the jog corners $O$, again producing continuous plastic flow. This type of dynamic spiral source was not seen in Ref. [30], since the middle segment of superjog was sessile and cross-slip was not considered in their simulation. As illustrated in Figure 4.8, the joint points, $O_{1}$ and $O_{2}$ in superjog $A O_{1} O_{2} B$, and $O$ in the dynamic spiral sources $A O B$, moved along the intersection line of the two intersected slip planes. When these joint points moved close to the free surface with its attractive image forces, they 

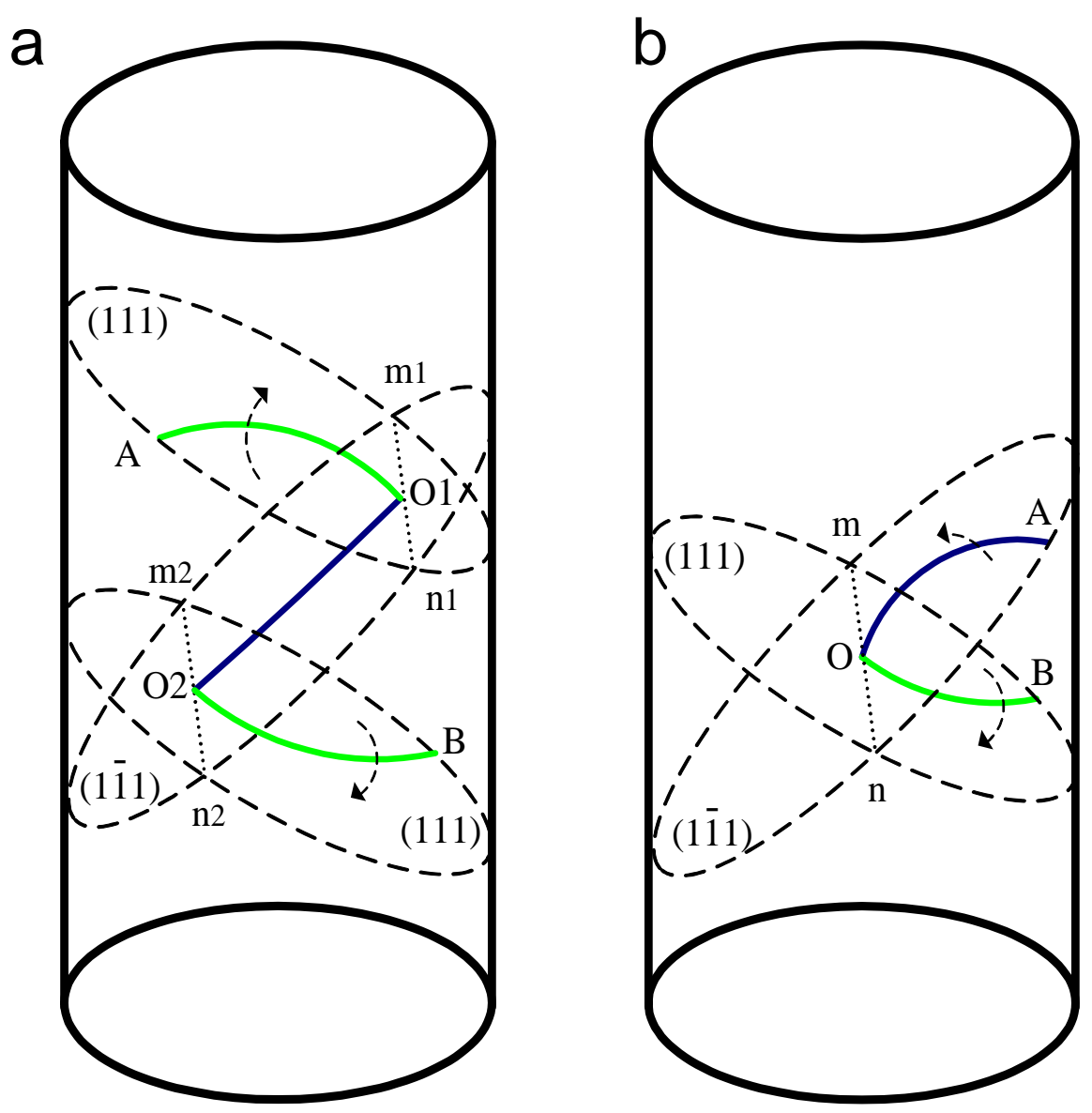

Figure 4.8 Configuration of superjog and dynamic spiral source: green line with $1 / 2[\overline{1} 01](111)$ and navy line with $1 / 2[\overline{1} 01](1 \overline{1} 1)$.

escaped and the dynamic spiral sources or superjogs ceased to operate. The movie B in supplementary materials of ref. [23] gives one example of flow intermittency as the moving dynamic spiral source escaped from free surface. The dynamic spiral source has two arms on different slip planes as shown in Figure $4.8 \mathrm{~b}$. With increasing load, they could operate independently on their own slip planes, and the joint point could move along the intersection line of the two slip planes. The stability of this dynamic source depends on the exact position of the jog corner and the sample diameter. For this source, after operating several times and 
generating a certain amount of plastic strain, it gradually escaped from the free surface and ceased to operate. Since there were no other operating sources, to sustain the applied strain rate required that the elastic strain (linearly related to the applied stress) increased until another source could be activated. During this period, the fraction of plastic strain in the total strain approached zero (no operating sources) and the strain hardening part was thus essentially elastic. This dislocation-starved condition (the shutting off of available dislocation sources) is called "exhaustion hardening", and is found both in experiments and simulations $[16,27]$. After the applied stress increased to a sufficiently high level, new sources were activated, generating plastic strain. Again, to keep the same overall strain rate, the elastic strain (applied stress) stopped increasing, leading to a plateau in the stress-strain curve corresponding to continuous operation of this new source. This type of dynamic source showed considerable variability in behavior. In some cases, the sources just operated several times and then escaped to the surface. While in others they were stable and operated numerous times, existing as long as the simulations were run. Thus, the degree of "exhaustion hardening" caused by the destruction of dynamic sources cannot be predicted $a$ priori and requires knowing the details of the internal dislocation structures. We can say, however, that the frequency of this mechanism is much higher in smaller samples, in which the dynamic sources are more easily destroyed at the surface and then regenerated.

The size-dependent exhaustion processes also affect the usual forest-hardening processes of junction formation and dipole interactions, resulting in the shutting off of already scarce dislocation sources. Figure 4.9 shows two typical cases of junction formation and collinear reaction, which leads to intermittent plastic flow. This mechanism has been observed previously by Rao and coworkers [22]. In Figure 4.9a, the single-ended spiral source S1 sweeps in its slip plane until it meets the FR source $S 2$. As $S 1$ moves close to $S 2$, a glissile junction was formed, locking the dislocations as shown in Figure $4.9 \mathrm{~b}$ and $\mathrm{c}$. When the applied stress is increased to a critical value, the glissile junction unzipped and the spiral 
a

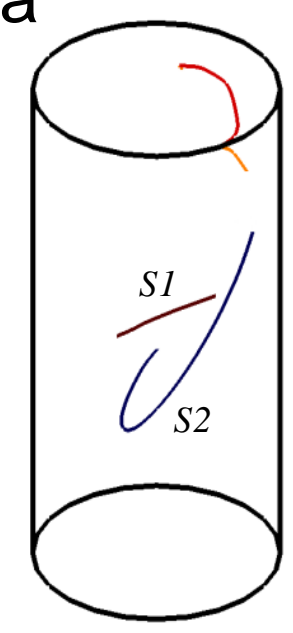

e

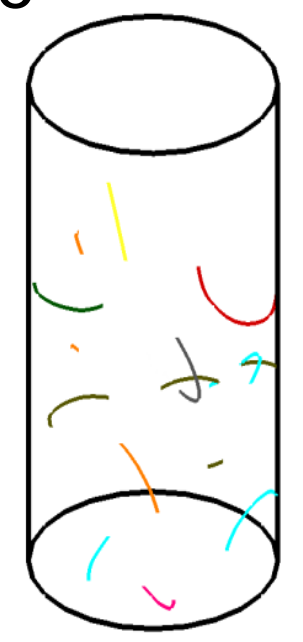

b

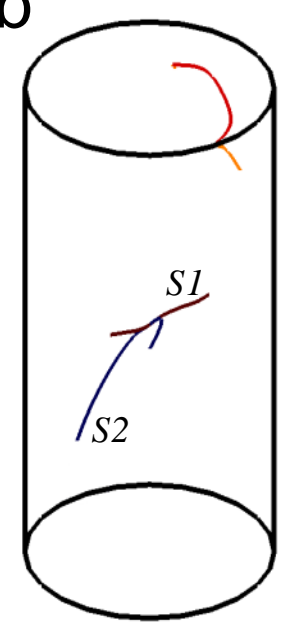

f

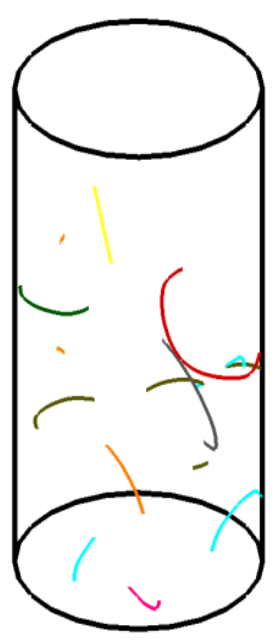

C
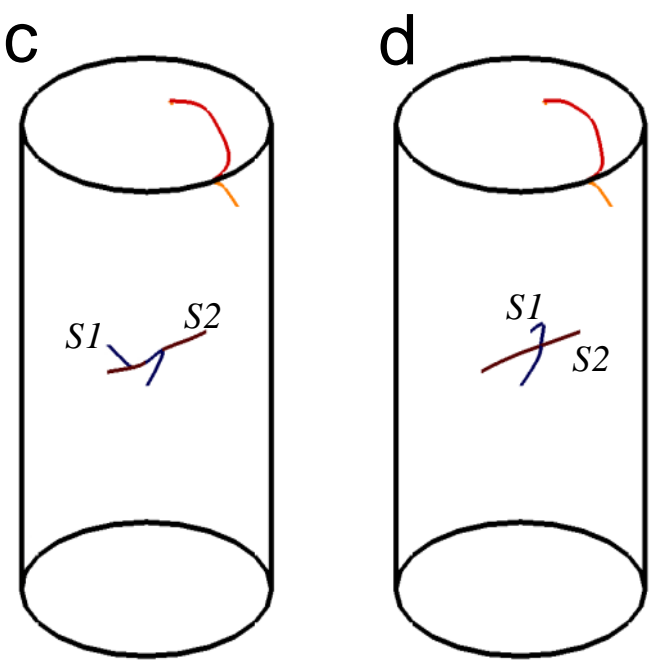

g

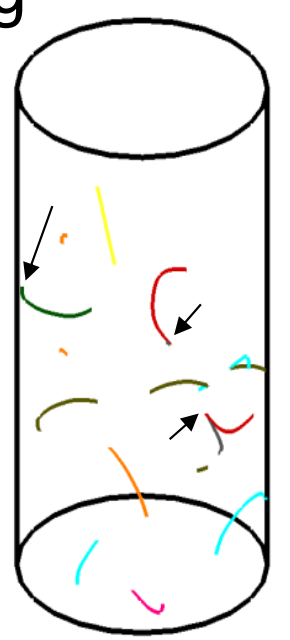

h

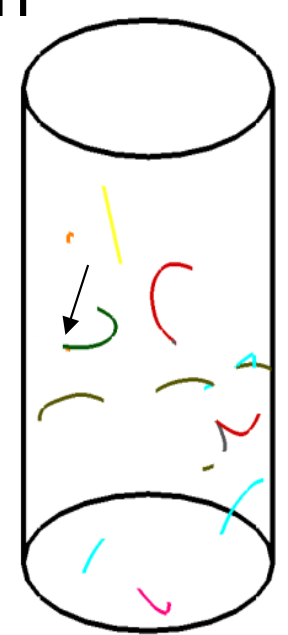

Figure 4.9 Dislocation reactions causing flow intermittence: (a-d) glissile junction, brown line with $1 / 2[\overline{1} 01](111)$ and navy line with $1 / 2[\overline{1} 01](1 \overline{1} 1),(\mathrm{e}-\mathrm{h})$ collinear reaction, grey line with $1 / 2[01 \overline{1}](\overline{1} 11)$ and red line with $1 / 2[01 \overline{1}](111)$.

source $S 1$ cyclically rotated around the pinning point and created continual plastic strain for the sample in Figure 4.9d. In contrast to glissile junction, the collinear reaction formed by two mobile spiral sources in Figure 4.9e-h was much stronger and could not be easily 
dissolved, so the new dislocation source was activated in Figure $4.9 \mathrm{~h}$ after the loading increased.

In Ref. [31], strain bursts are attributed to the destruction of jammed configurations by long-range interactions, which produce a collective avalanche-like process. This mechanism seems to be at least somewhat consistent with our observations, as shown in movie A in ref. [23] and Figure 4.9. The destruction of simple junctions leads to relatively small strain bursts as the released free dislocations quickly escape to the surface. However, the spiral sources released from the junction in Figure 4.9 continuously sweep in the slip plane and produce large strain bursts. These strain bursts, or avalanche-like processes, are strongly influenced by their physical size. As illustrated in movie B in ref. [23], the dynamic sources continuously create plastic strain under loading, with the amount of this strain dependent on their position and the sample diameter. From a statistical perspective, the probability of sources truncated by a surface increases with decreasing diameter. Thus the frequency of strain bursts and consequent flow intermittency in smaller samples is much higher than in larger samples, which is verified in both experiment and our simulation results. After the operation of dynamic sources is terminated by a surface, new sources need to be activated at a higher load level to generate continuous plastic deformation. Recently, Ngan et al. demonstrated that discrete strain bursts were directly related to the escape of dislocation sources to the sample surface [32], agreeing well with our simulation results and providing a physical explanation of the experimentally observed staircase stress-strain behavior.

\subsection{Size effects}

In Figure 4.10a, we show a series of stress-strain curves from samples with different diameters under uniaxial compression in the absence of loading gradients. These results show pronounced dependence on size, with smaller samples having higher strength. The stress shows discrete jumps accompanied by strain bursts of varying sizes before ending at a 

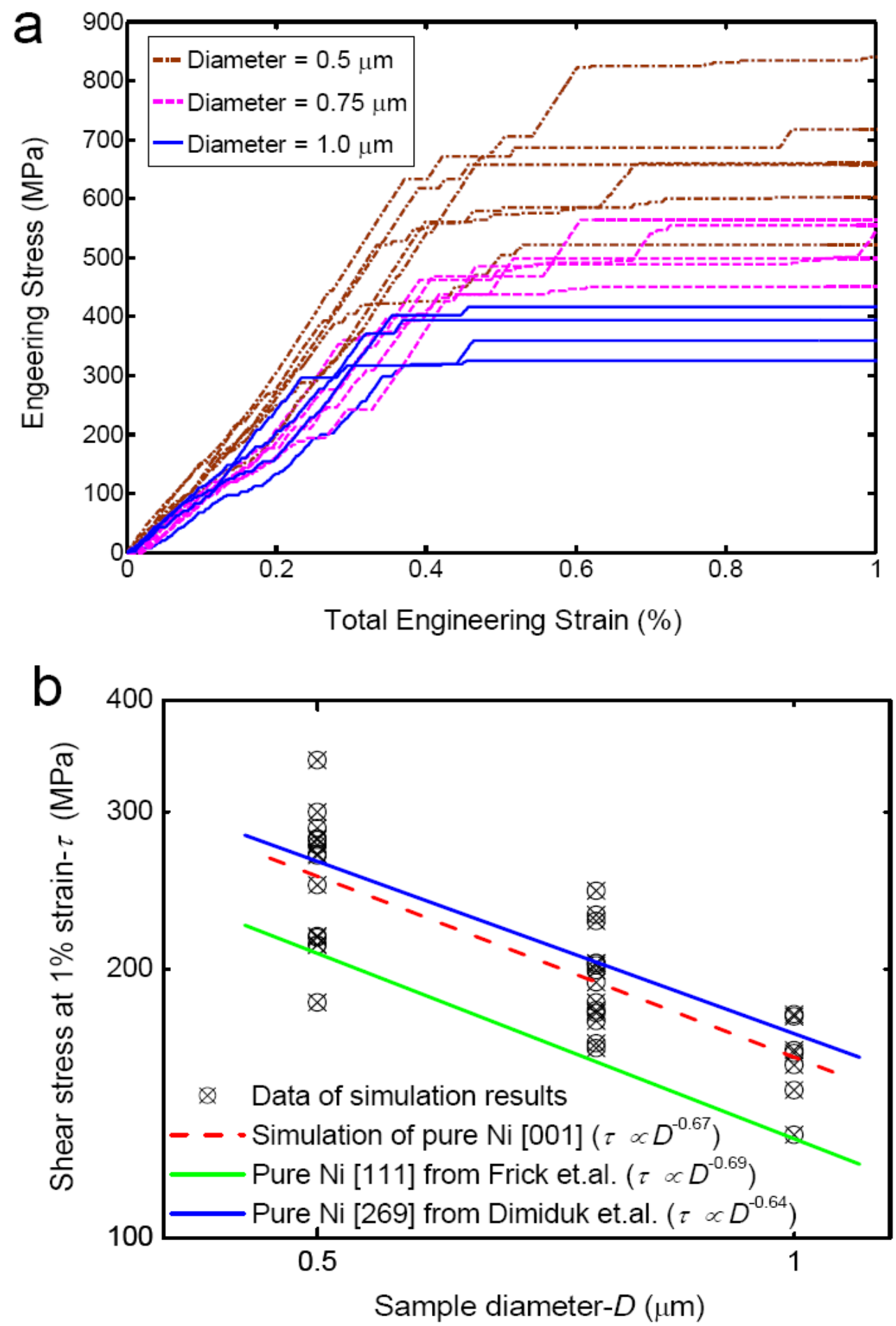

Figure 4.10 (a) Stress-strain curves obtained from simulation with different sizes, (b) comparison $\log \log$ plot of the shear stress at $1 \%$ total strain of simulation results and experimental results. 
saturation flow stress. There is a significant scatter in the magnitude of the saturation flow stress with decreasing diameter. All of these features of the compression stress-strain curves are in qualitative agreement with the experimentally observed behavior that shows discrete strain bursts separated by intervals of nearly elastic loading [13-15, 33-43].

In Figure $4.10 \mathrm{~b}$, the variation of the shear stress at $1 \%$ strain $(\tau)$ as a function of the sample diameter (D) are plotted on a logarithmic scale in both coordinates, for all simulations. The scatter in strength increases with decreasing sample size, largely because the mechanical response of smaller samples depends on a single or, at most very few, active sources. We fit the average value of $\tau$ for each size to a function of the form $\tau \propto \mathrm{D}^{-\mathrm{n}}$ and find a scaling exponent $\mathrm{n} \approx 0.67$. Similar behavior in both the magnitude and scatter of the values for the shear stress at $1 \%$ strain was seen experimentally, with an exponent of 0.64 under [269] single-slip loading from Ref. [13] and 0.69 under [111] multi-slip loading from Ref. [34].

In bulk samples, Taylor's hardening law, which states that the flow stress is proportional to the square root of the dislocation density, has been confirmed by both theoretical and experimental studies [44]. However, there is little size dependence of the evolution of the dislocation density, since all samples showing similar dislocation density variations as shown in Figure 4.6. Thus, Taylor's law does not hold and cannot be used to develop a theory of the size effects of plasticity in small volumes.

Recently, Parthasarathy et al. [45] developed a statistical model for the flow strength of small samples, which was completely based on the stochastics of spiral source (single-arm source) lengths in samples of finite size. In their studies, the spiral source with one permanent inside pinning point could be formed either by the FR sources being truncated at the free surface or directly generated in the initial structure of simulation. In either case, the spiral sources have a minimum strength based on the relative distance between the sources and the free surfaces. For the FR sources, the minimum always appears when the FR source 


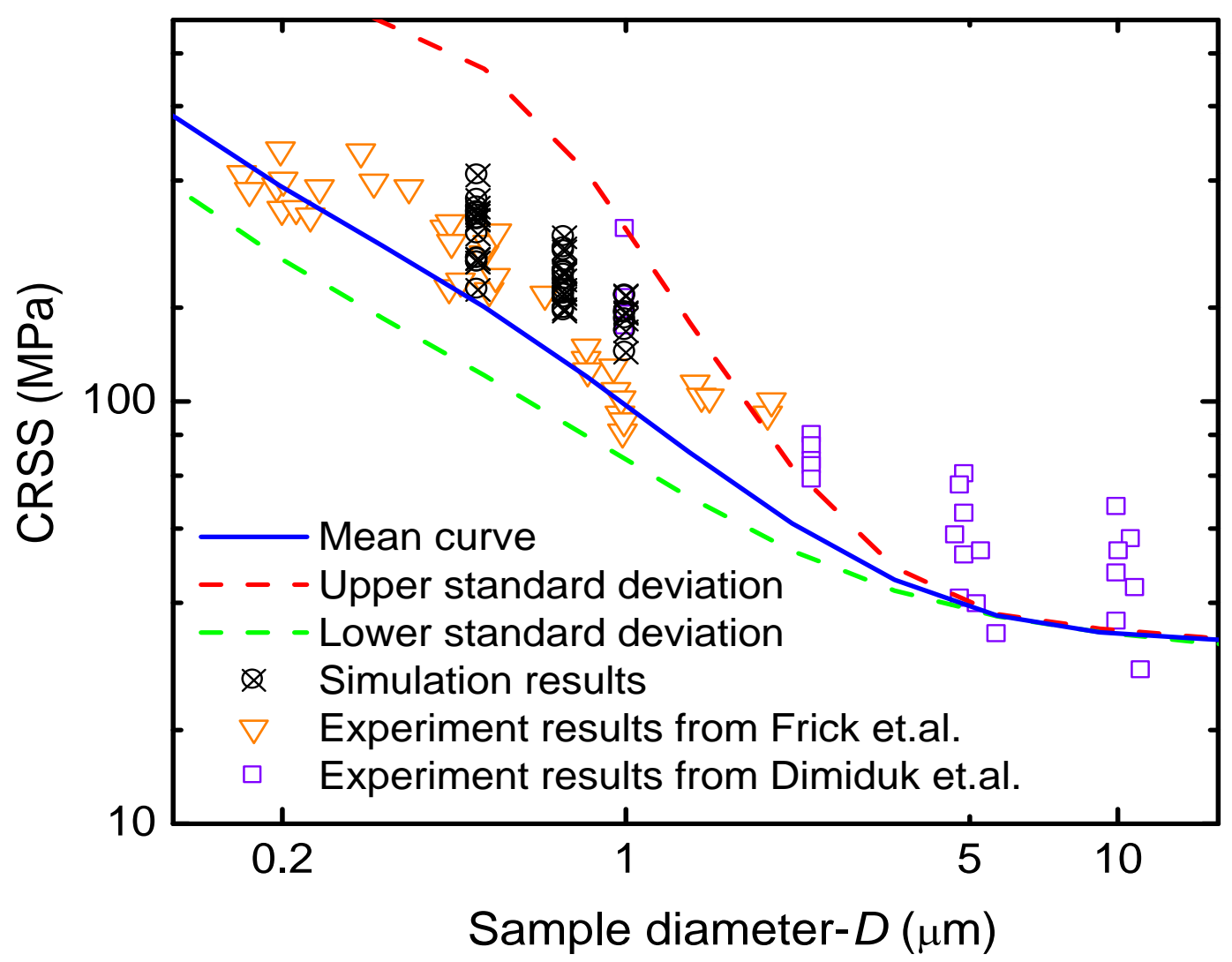

Figure 4.11 Comparison log-log plot of the statistic model and simulation and experimental results.

is set at the center of the sample and with the length of around 1/3 the slip-plane characteristic dimension [46]. For a single-arm source, the minimum is set with the source pinning point at the center of the sample [45]. This stochastic model was validated by the in-situ observation of dislocation behavior in a submicrometre single crystal in which single-ended sources are limited approximately by half of the crystal width [47].

Since the flow stress was always determined by the strength of spiral sources or stable dynamic sources in our simulations, we used our simulation results and experiment results from references $[13,34]$ to compare with this stochastic model, which estimates the critical resolved shear stress (CRSS) as following: 


$$
\tau=\tau_{o}+k_{s} \mu \frac{\ln (\bar{\lambda} / b)}{(\bar{\lambda} / b)}+k_{f} \mu b \sqrt{\rho_{f}} \ln \left(\frac{1}{b \sqrt{\rho_{f}}}\right)
$$

where $\tau_{o}$ is the lattice friction stress (11 MPa for $\left.\mathrm{Ni}\right), k_{s}$ is a source-hardening constant, with magnitude $k_{s}=0.12$, derived through a recent study [3], $k_{f}$ represents the hardening coefficient using a value of $k_{f}=0.061$ [48], $\rho_{f}$ is forest dislocations density, $\rho_{f}=2 \times 10^{12} \mathrm{~m}^{-2}$ and $\bar{\lambda}$ is an average effective source length calculated from the statistic model [45]. The second and third term in equation (5) represent source truncation strengthening [28] and forest strengthening, respectively. It can be seen from Figure 4.11 that this single-arm model could predict the initial stress for plasticity well for smaller samples, because only one or at most a few mobile dislocations determine the strength at small volumes, agreeing with the basic assumption in this model. For the larger samples, the predicted scatter is less than that observed, since internal dislocation structures and reactions are more complicated in larger samples than those in smaller ones.

\subsection{Concluding remarks}

Experimental-like initial dislocation structures cut from larger deformed samples have been introduced into 3D DD SIMULATIONS to study the plasticity in small sizes. Image forces from traction-free surface and as well as thermally-activated cross-slip were considered in our study. Three different sizes of micropillars all with initially relaxed dislocation densities around $2.0 \times 10^{13} \mathrm{~m}^{-2}$ have been analyzed under uniaxial compression to identify the relationship between the evolution of internal dislocation structure and overall mechanical behaviors.

The results indicate that the loading direction has negligible effect on the flow stress with both multi-slip and single-slip loading resulting in the similar saturation. This lack of a 
dependence on loading direction can be easily understood. Since the number of dislocation sources decreases with the sample diameters, the probability to activate a source with low Schmid factors increases in small samples.

In small samples, dynamic sources can be easily generated by cross-slip or collinear reactions, the stability of which depends on the position and sample size. There were at least two origins of "exhaustion hardening": the escape of dynamic sources from the surface and dislocation interactions such as junction formation. Both of these effects shut off the activated sources, leading to the flow intermittency. The "mechanical annealing" at the early stage of deformation were seen to arise from the surface dislocations and the weakly-entangled dislocations leaving the sample. The drop in dislocation density was followed by an increase that always resulted from processes that were enabled by cross-slip. The scarcity of available dislocation sources gives a major contribution to the higher flow stress and larger scatter of strength in smaller sizes. The scaling law determined from the current simulation results is close to that found experimentally.

There are still many unanswered questions regarding size-dependent strengthening in small volumes, such as the critical size for transition from bulk behavior and the role that dislocation structures and mechanisms play in determining that critical size. Further investigations are planned for larger samples based on our simulation framework to address these questions. Our goal is to develop a more sophisticated model to predict the mechanical behavior of microcrystals over a wide range of sizes. 


\section{References}

[1] Wang ZQ, Beyerlein IJ, Lesar R. Plastic anisotropy in fcc single crystals in high rate deformation. International Journal of Plasticity 2009;25:26.

[2] Hirth JP, Lothe J. Theory of Dislocations McGraw-Hill, New York, 1982.

[3] Puschl W. Models for dislocation cross-slip in close-packed crystal structures: a critical review. Prog. Mater. Sci. 2002;47:415.

[4] Kubin LP, Canova G. The modeling of dislocation patterns. Scripta Metallurgica Et Materialia 1992;27:957.

[5] Verdier M, Fivel M, Groma I. Mesoscopic scale simulation of dislocation dynamics in fcc metals: Principles and applications. Model. Simul. Mater. Sci. Eng. 1998;6:755.

[6] Escaig B. In: A. Rosenfield, G. Hahn, A. Bement and R. Jaffee, Editors. Proceedings of the Battelle colloquium in dislocation dynamics. New York McGraw-Hill, 1968.

[7] Friedel J. In: J. Fisher, W. Johnston, R. Thromson and T. Vreeland, Editors. Dislocations and mechanical properties of crystals. New York Wiley-Interscience, 1957.

[8] Rao S, Parthasarathy TA, Woodward C. Atomistic simulation of cross-slip processes in model fcc structures. Philos. Mag. A-Phys. Condens. Matter Struct. Defect Mech. Prop. 1999;79:1167.

[9] Starenchenko VA, Lychagin DV, Shaekhov RV, Kozlov ÉV. Action of test temperature on evolution of dislocation structure of nickel single crystals with the [001] compression axis. Russian Physics Journal 1999;42:653.

[10] Wang ZQ, Beyerlein IJ, Lesar R. The importance of cross-slip in high-rate deformation. Model. Simul. Mater. Sci. Eng. 2007;15:675.

[11] Norfleet DM, Dimiduk DM, Polasik SJ, Uchic MD, Mills MJ. Dislocation structures and their relationship to strength in deformed nickel microcrystals. Acta Materialia 2008;56:2988.

[12] Uchic MD, Dimiduk DM, Florando JN, Nix WD. Sample dimensions influence strength and crystal plasticity. Science 2004;305:986.

[13] Dimiduk DM, Uchic MD, Parthasarathy TA. Size-affected single-slip behavior of pure nickel microcrystals. Acta Materialia 2005;53:4065. 
[14] Dimiduk DM, Uchic MD, Rao SI, Woodward C, Parthasarathy TA. Overview of experiments on microcrystal plasticity in FCC-derivative materials: selected challenges for modelling and simulation of plasticity. Model. Simul. Mater. Sci. Eng. 2007; $15: 135$.

[15] Dimiduk DM, Woodward C, LeSar R, Uchic MD. Scale-free intermittent flow in crystal plasticity. Science 2006;312:1188.

[16] Motz C, Weygand D, Senger J, Gumbsch P. Initial dislocation structures in 3-D discrete dislocation dynamics and their influence on microscale plasticity. Acta Materialia 2009;57:1744.

[17] Senger J, Weygand D, Gumbsch P, Kraft O. Discrete dislocation simulations of the plasticity of micro-pillars under uniaxial loading. Scr. Mater. 2008;58:587.

[18] Tang H, Schwarz KW, Espinosa HD. Dislocation escape-related size effects in single-crystal micropillars under uniaxial compression. Acta Materialia 2007;55:1607.

[19] Weygand D, Poignant M, Gumbsch P, Kraft O. Three-dimensional dislocation dynamics simulation of the influence of sample size on the stress-strain behavior of fcc single-crystalline pillars. Materials Science and Engineering a-Structural Materials Properties Microstructure and Processing 2008;483:188.

[20] Aliabadi MH. The Boundary Element Method : Applications in Solids and Structures Wiley 2002.

[21] Ng KS, Ngan AHW. Breakdown of Schmid's law in micropillars. Scr. Mater. 2008;59:796.

[22] Rao SI, Dimiduk DM, Parthasarathy TA, Uchic MD, Tang M, Woodward C. Athermal mechanisms of size-dependent crystal flow gleaned from three-dimensional discrete dislocation simulations. Acta Materialia 2008;56:3245.

[23] Zhou C, Biner SB, LeSar R. Discrete dislocation dynamics simulations of plasticity at small scales. Acta Materialia 2010;58:1565.

[24] Weinberger CR, Cai W. Surface-controlled dislocation multiplication in metal micropillars. Proc. Natl. Acad. Sci. U. S. A. 2008;105:14304.

[25] Bei H, Shim S, Pharr GM, George EP. Effects of pre-strain on the compressive stress-strain response of Mo-alloy single-crystal micropillars. Acta Materialia 2008;56:4762. 
[26] Shim S, Bei H, Miller MK, Pharr GM, George EP. Effects of focused ion beam milling on the compressive behavior of directionally solidified micropillars and the nanoindentation response of an electropolished surface. Acta Materialia 2009;57:503.

[27] Shan ZW, Mishra RK, Asif SAS, Warren OL, Minor AM. Mechanical annealing and source-limited deformation in submicrometre-diameter $\mathrm{Ni}$ crystals. Nat. Mater. 2008;7:115.

[28] Rao SI, Dimiduk DM, Tang M, Parthasarathy TA, Uchic MD, Woodward C. Estimating the strength of single-ended dislocation sources in micron-sized single crystals. Philosophical Magazine 2007;87:4777.

[29] Madec R, Devincre B, Kubin L, Hoc T, Rodney D. The role of collinear interaction in dislocation-induced hardening. Science 2003;301:1879.

[30] Tang H, Schwarz KW, Espinosa HD. Dislocation-source shutdown and the plastic behavior of single-crystal micropillars. Physical Review Letters 2008;100.

[31] Csikor FF, Motz C, Weygand D, Zaiser M, Zapperi S. Dislocation avalanches, strain bursts, and the problem of plastic forming at the micrometer scale. Science 2007;318:251.

[32] $\mathrm{Ng} \mathrm{KS}$, Ngan AHW. Effects of trapping dislocations within small crystals on their deformation behavior. Acta Materialia 2009;57:4902.

[33] Brinckmann S, Kim JY, Greer JR. Fundamental differences in mechanical behavior between two types of crystals at the nanoscale. Physical Review Letters 2008;100.

[34] Frick CP, Clark BG, Orso S, Schneider AS, Arzt E. Size effect on strength and strain hardening of small-scale 111 nickel compression pillars. Materials Science and Engineering a-Structural Materials Properties Microstructure and Processing 2008;489:319.

[35] Greer JR. Bridging the gap between computational and experimental length scales: A review on nanoscale plasticity. Reviews on Advanced Materials Science 2006;13:59.

[36] Greer JR, Nix WD. Size dependence of mechanical properties of gold at the sub-micron scale. Applied Physics a-Materials Science \& Processing 2005;80:1625.

[37] Greer JR, Nix WD. Nanoscale gold pillars strengthened through dislocation starvation. Physical Review B 2006;73.

[38] Greer JR, Oliver WC, Nix WD. Size dependence of mechanical properties of gold at the micron scale in the absence of strain gradients. Acta Materialia 2005;53:1821. 
[39] Ng KS, Ngan AHW. Creep of micron-sized aluminium columns. Philos. Mag. Lett. 2007;87:967.

[40] Ng KS, Ngan AHW. Stochastic nature of plasticity of aluminum micro-pillars. Acta Materialia 2008;56:1712.

[41] Ng KS, Ngan AHW. Stochastic theory for jerky deformation in small crystal volumes with pre-existing dislocations. Philosophical Magazine 2008;88:677.

[42] Uchic MD, Shade PA, Dimiduk DM. Plasticity of Micrometer-Scale Single Crystals in Compression. Ann. Rev. Mater. Res. 2009;39:361.

[43] Volkert CA, Lilleodden ET. Size effects in the deformation of sub-micron Au columns. Philosophical Magazine 2006;86:5567.

[44] Kocks UF, Mecking H. Physics and phenomenology of strain hardening: the FCC case. Prog. Mater. Sci. 2003;48:171.

[45] Parthasarathy TA, Rao SI, Dimiduk DM, Uchic MD, Trinkle DR. Contribution to size effect of yield strength from the stochastics of dislocation source lengths in finite samples. Scr. Mater. 2007;56:313.

[46] von Blanckenhagen B, Gumbsch P, Arzt E. Dislocation sources and the flow stress of polycrystalline thin metal films. Philos. Mag. Lett. 2003;83:1.

[47] Oh SH, Legros M, Kiener D, Dehm G. In situ observation of dislocation nucleation and escape in a submicrometre aluminium single crystal. Nat. Mater. 2009;8:95.

[48] Basinski ZS. Forest hardening in face-centered cubic metals. Scripta Metallurgica 1974;8:1301. 


\section{CHAPTER 5}

\section{PLASTIC DEFORMATION MECHANISMS OF FCC SINGLE \\ CRYSTALS AT SMALL SCALES}

Starting with the pioneering microcompression measurements of Uchic et al. that reported an anomalous increase in the strength of micron-scale single crystal pillars as their diameter decreased[1], numerous research groups have observed similar size effects in various FCC single crystals[2-14]. An inverse relationship between sample size and flow stress was predicted by strain gradient models for small indentations, resulting from an increase in geometrically necessary dislocation densities to accommodate the lattice mismatch [15-16]. However, TEM investigations [17] revealed that the dislocation structure on the active slip systems in micropillars with diameters larger than $2 \mu \mathrm{m}$ is comparable to that found in bulk samples deformed to a similar state. Furthermore, the dislocation density in nanopillars smaller than $150 \mathrm{~nm}$ apparently approaches zero after deformation [18]. These experimental observations indicate that mechanisms other than the gradient-induced storage of geometrically necessary dislocations must be the cause of the observed size effects in microcompression tests on micropillars.

Two basic models have been used to explain the size effects in plasticity in FCC single crystals. The first is the "dislocation starvation" (DS) model [4-6, 12, 19-20], in which dislocations can easily escape from nearby free surfaces in a small sample prior to dislocation multiplication, leaving samples in a dislocation-free state. Continuous plastic flow would then require an increase in applied load to nucleate dislocations at the surface. Thus, the principal idea behind the DS model is that plastic deformation at small scale is dislocation nucleation dominated. The other model is the "single-arm dislocation" (SAD) model, which is based on the notion that size effects in the plasticity of small single crystals can be 
rationalized almost completely by considering the stochastics of single-arm dislocation source lengths in the sample [22]. In contrast with the DS model, the SAD model assumes that the plastic deformation is induced by multiplication of internal dislocation sources rather than nucleation of surface dislocations. To identify which of the two models best describes the dislocation behavior requires a better understanding of the evolution of dislocation structures with increasing strain and the details of the dynamic behavior of internal dislocation sources.

3-D dislocation dynamics (DD) simulations, in which dislocations are the simulated entities, have been the primary modeling tool employed to study the various aspects of plastic behavior in nano- and micro-samples. The first applications of 3-D DD simulations to this problem employed a set of isolated Frank-Read sources (FRs) with rigidly fixed ends as the starting dislocation populations [21-26]. To extend that simple (and limited) model, Tang et al. [27] employed artificially-generated jogged dislocations as the initial dislocation configuration for their simulations (though they neglected the image stresses and cross-slip) and demonstrated that the shut-down of sources causes staircase behavior similar to that observed in experiments. Motz et al. [28] used the dislocation structures relaxed from a high density of closed dislocation loops as the initial input for their simulations. In our previous work, we [14] employed experimental-like initial dislocation structures, which were created by cutting the cylindrical sample from the results of simulations on larger, bulk, samples. Our goal was to mimic the physics of real systems as closely as possible. To that end, we also included a boundary element method to determine the image forces and a detailed model of cross slip. We found that the scarcity of available dislocation sources is indeed a major contributor to the higher flow stress in smaller sizes [29]. Despite this progress, however, further studies are needed both to determine the critical events for plastic deformation at small scales and to derive more accurate and reliable models to predict the mechanical properties of small scale materials. 
In this paper, we present results from 3-D DD simulations on the dynamic behavior of internal dislocation sources in micropillars. Based on our simulation results, analytical formulations of the dislocation starvation (DS) model and a general single-arm dislocation (SAD) model were developed, from which we can identify the relationship between nucleation-dominated and multiplication-dominated plastic deformation in small-scale FCC single crystals.

\subsection{Simulation procedures}

We employed the parametric DD method described in detail in [30-32] to simulate the mechanical behavior of Ni single crystals under uniform compression. For the simulations in this work, the material properties of $\mathrm{Ni}$ are used: shear modulus $\mu=76 \mathrm{GPa}$, Poisson's ratio $v$ $=0.31$, and lattice constant $\mathrm{a}=0.35 \mathrm{~nm}$.

A sophisticated thermally-activated cross-slip model developed by Kubin and co-workers [33-34] was adopted in our DD simulations. We employed a Monte Carlo method to determine the activation of cross slip based on the probability of the cross slip of a screw segment with length $L$ in a discrete time step, which is determined by an activation energy $V_{a c t}\left(|\tau|-\tau_{I I I}\right)$ and the resolved shear stress on the cross-slip plane $\tau$,

$$
P=\beta \frac{L}{L_{0}} \frac{\delta t}{\delta t_{0}} \exp \left[-\frac{V_{a c t}}{k T}\left(|\tau|-\tau_{I I I}\right)\right] \text {, }
$$

where $\beta$ is a normalization constant, $k$ is the Boltzmann constant, $T$ is set to room temperature, $V_{a c t}$ is the activation volume, and $\tau_{I I I}$ is the stress at which stage-three hardening starts. In $\mathrm{Ni}, V_{a c t}$ is equal to $420 b^{3}$ with $b$ the magnitude of Burgers vector, $\tau_{I I I}=55 \mathrm{MPa}$ [35-36], and $L_{0}=1 \mu \mathrm{m}$ and $\delta t_{0}=1 \mathrm{~s}$ are reference values for the length of the cross-slipping segment and for the time step. 
In our computations, the experimental loading conditions of Dimiduk and coworkers $[1-2,17]$ were simulated, in which a mixture of constant displacement rate and creep-like loading conditions were employed; the applied stress was discretely increased by a small fixed value $(\delta \sigma)$ every time the plastic strain rate approached the applied strain rate. In all simulations, compression loading was performed in the [001] direction. When the plastic strain rate was equal to 50 , the applied load was increased by $1.0 \mathrm{MPa}$, i.e. $\delta \sigma=1.0 \mathrm{MPa}$, for $\dot{\varepsilon}^{p}=50$, while the applied stress was kept constant when the plastic strain rate was larger than 50, i.e. $\delta \sigma=0$, for $\dot{\varepsilon}^{p}>50$.

\subsection{Stability of internal dislocation sources}

The major question that has arisen from recent work on plasticity at small scales is whether the activated dislocations producing continuous plastic strain are the pre-existing internal sources or sources nucleated from the surface $[4-6,10-12,20]$. If the dislocation starvation mechanism is operant, nucleation of surface dislocation sources is the most likely contributor to continuous plastic flow. Otherwise, plastic deformation is likely to be dominated by the multiplication of internal dislocation sources. To answer this question requires an examination of balance between the stability of internal dislocation sources and the probability of dislocation starvation in samples with different sizes. In recent in situ TEM studies, Oh et al. observed that some internal dislocation sources can be naturally created by cross-slip of dislocations near free surfaces [37-38]. Unfortunately, it is unclear how stable those naturally formed sources are in different samples. If these sources have a short enough lifetime such that they can only operate a few times before finally escaping from the free surfaces, then dislocation nucleation from the surface likely plays a critical role in controlling plastic deformation.

To study the formation and stability of internal dislocation sources, we mimicked the nucleation of a dislocation by putting a surface dislocation with a length of $100 \mathrm{~nm}$ on the 
surface and lying along the $\frac{1}{2}[01 \overline{1}](111)$ slip system in micropillars with various diameters and an aspect ratio fixed at $D: H=1: 3$ (as observed in in situ experiments) [37]. All surface dislocations nucleated from the middle part of the micropillars and were set as near-screw dislocations to increase the cross-slip probability. Since we do not know the actual stress required to nucleate dislocations from a rough surface, we estimated the nucleation stress based on $\gamma / b$, where the Burgers vector, $b=0.25 \mathrm{~nm}$ and the stacking fault energy, $\gamma=0.1$ $\mathrm{J} / \mathrm{m}^{2}$ for Ni [39]. Thus, the required nucleation stress on $\{111\}$ slip systems with Schmid factor equal 0.41 is approximately equal to $1000 \mathrm{MPa}$.

Figure 5.1 shows the sequential snapshots from a simulation in which image stresses at the free surfaces were ignored. In that case, a surface dislocation was nucleated from one side of the sample, quickly swept across the plane and exited from other side. When image stresses are included, the response is quite different, in that the screw segment on the activated dislocation cross slipped from the original plane to an adjacent plane and then emitted a new FR source, as shown in Figure 5.2 for a typical case in a $750 \mathrm{~nm}$ pillar. Figure
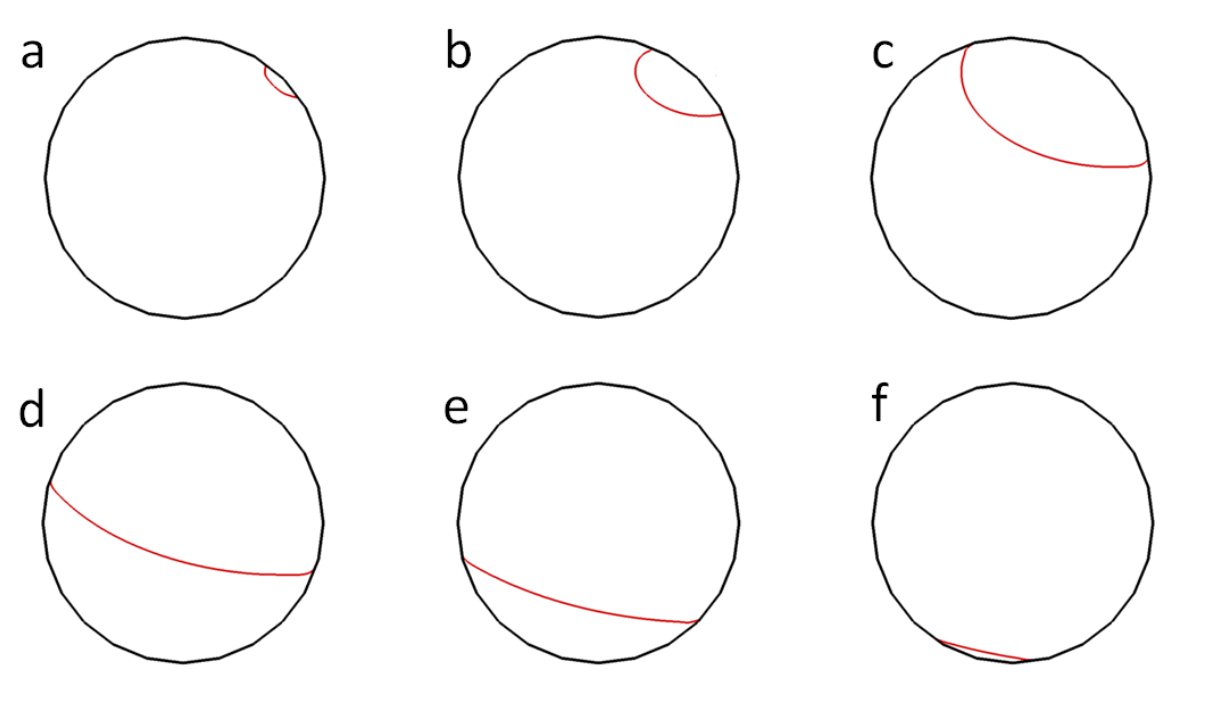

Figure 5.1 Dislocation Nucleating and escaping from the surface of micropillar without considering image stresses (viewing along the Z-direction). 
5.2a-d shows the nucleated dislocation gliding on the original (111) plane. In Figure 5.2e, the screw segment nearest the free surface has cross slipped to the (111) plane under the influence of image stresses and formed a new FR source with the same Burgers vector $\frac{1}{2}[01 \overline{1}]$ as the original one. The newly formed FR dislocation source contains two internal pinning points, $p 1$ and $p 2$, as shown in Figure 5.2e. The pinning points have two single-arms on different slip planes with the same Burgers vector and can move along the intersection line of the two slip planes [27, 29]. Under loading, the two dislocation arms will operate independently around the jog corners, producing continuous plastic flow. In Fig. $2 \mathrm{f}$ and $\mathrm{g}$, the newly formed source bowed out under the external loading and then was truncated by the free surface. Since the source containing pinning point $p 2$ was quite close to the free surface, it exited the pillar under attractive image forces and only the $p l$ source continued operating, as shown in Figure 5.2h. One of the new dislocations generated by the source $p l$ moved in the opposite direction from the original one (Figure 5.2i) and when it approached the surface, a cross-slip process similar to that in Figure 5.2e occurred, in which one new FR source was formed, as shown in Figure 5.2j. The whole process in Figure 5.2 illustrates how internal
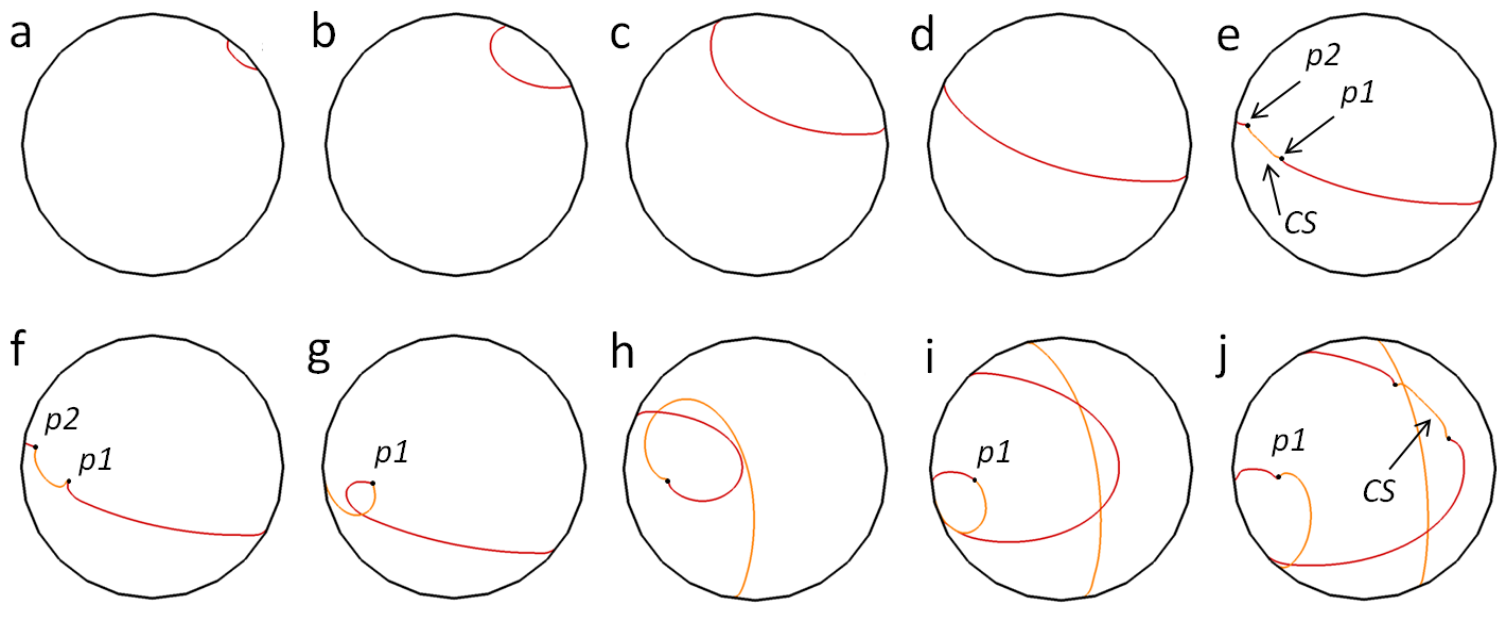

Figure 5.2 Dislocation nucleating from the surface and forming internal pinning points by cross-slip (CS) under the influence of image stresses (viewing along the Z-direction). 
sources can be formed by cross-slip under the influence of external stress fields. In FCC crystals, cross-slip of dislocations mainly depends on the local resolved shear stresses and dislocation line directions. In small volumes with large surface to volume ratios, image stresses from surfaces can alter the local resolved shear stresses on slip planes, which results in an increase in the probability of cross-slip. In our simulations, we find that internal sources formed by cross-slip of the activated surface dislocation are most likely if the surface dislocation initially nucleated from a direction within $\pm 15^{\circ}$ from the direction of the pure screw dislocation. The qualitative behavior reported here is robust against modification of simulation details such as the discretized length of dislocation segments. It is worth noting that the current DD simulation results differ from recent atomistic simulations [40], in which cross-slip of dislocations seldom happened and, thus, nucleation of surface dislocations controlled the plastic flow. However, since the sample size in the atomistic simulations is only $36 \mathrm{~nm}$ and the applied strain rate is seven orders of magnitude larger than those in our simulations, it is perhaps not surprising that the simulations differ.

To study the stability and effectiveness of the naturally formed sources, the dislocation density for samples of different sizes along with the corresponding stress-strain curves are plotted in Figure 5.3a. The plastic strain produced by the nucleated surface dislocation and subsequently emitted sources decreases with the sample size, likely because dislocation sources in smaller samples have shorter residence lifetime than those in larger samples under the influence of attractive image forces and confined geometries. When all the dislocations have been driven out from surfaces, the samples arrive at the state of dislocation starvation. The movie in the supplementary materials gives one example of dislocation starvation in the $300 \mathrm{~nm}$ pillar that corresponds to the density-strain curve shown in Figure 5.3a. After dislocation starvation, additional dislocations must be nucleated for plasticity to commence, which requires the application of significantly higher stresses. During this period, the fraction of plastic strain in the total strain is zero (because of no dislocation sources) and the strain 

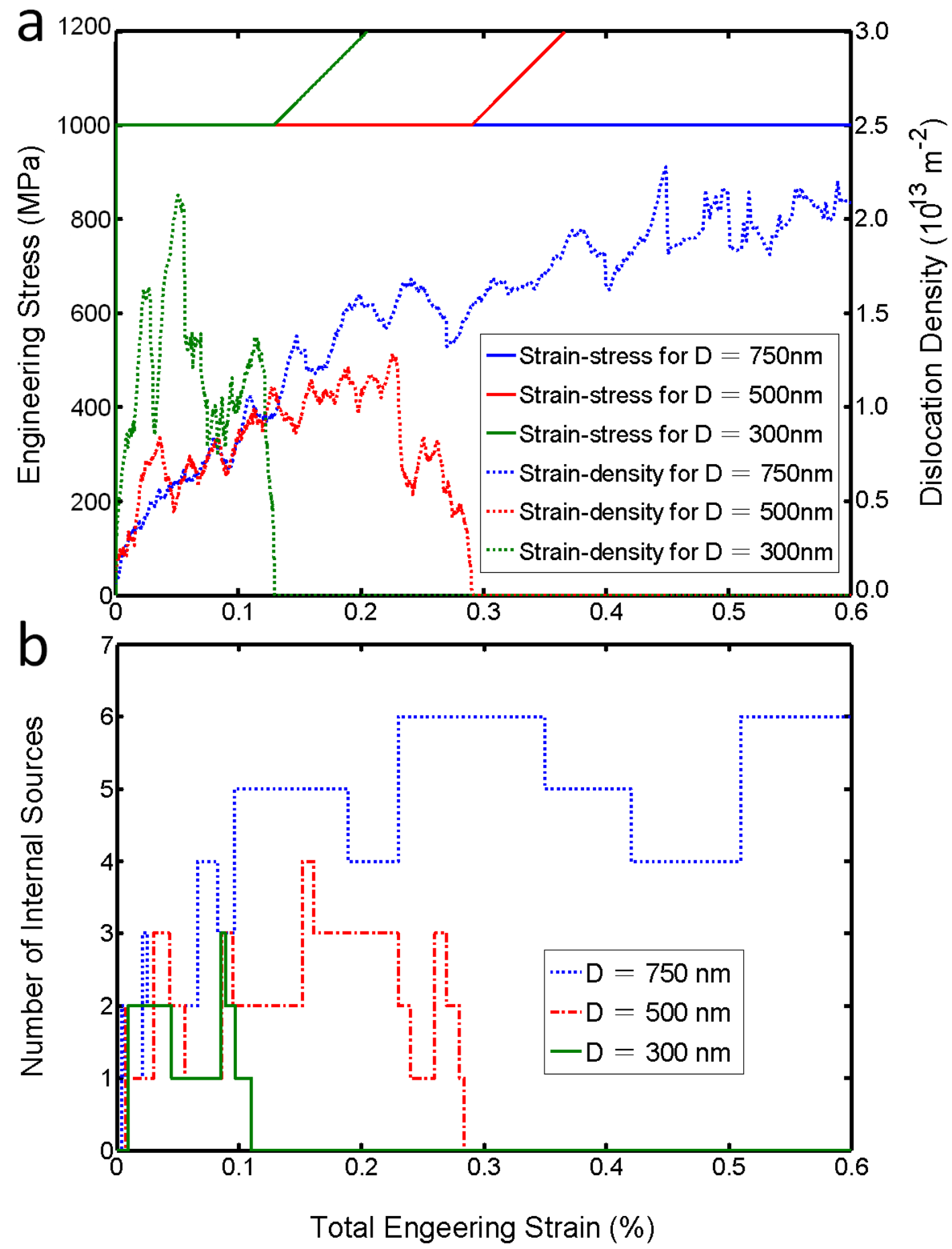

Figure 5.3 (a) Stress-strain curves and corresponding density-strain curves, (b) evolution of the number of internal dislocation sources. 
hardening is essentially elastic, as reflected in the stress-strain curves. This effect is called "starvation hardening." Since the dynamic sources have shorter residence lifetimes in smaller samples, the frequency of the repeating nucleating dislocation sources increases as the sample size decreases, which is one reasonable explanation for why smaller samples have a higher frequency of intermittency in plastic flow observed in most microcompression tests $[3-4,6,8,14]$. Figure $5.3 \mathrm{~b}$ plots the evolution of the number of internal dislocation sources for different sample sizes. Plastic flow is clearly a dynamic process, during which both loss and gain of dislocation sources can happen in all pillars at all sizes. However, the stability of these internal sources depends on the sample size, because those in the smaller samples have a shorter average distance to the free surfaces and thus it is easier for them to escape from the sample.

In real systems, internal dislocation sources are always present in the existing dislocation structures before further deformation. These structures are formed by dislocation reactions such as cross-slip, collinear annihilation [41], and Lomer-Cottrell junctions [28]. Assuming that the average length of dislocation sources in the sample scales with radius $R$ as $\langle L\rangle=s R$, the dislocation density in a micropillar with aspect ratio of $3: 1$, is equal to $s N R /\left(\pi R^{2} * 6 R\right)=$ $s N /\left(6 \pi R^{2}\right)$, where $N$ is the total number of dislocations. From this simple analysis, we can see that the increase of the total number of sources is 2 orders of magnitude faster than the increase in sample sizes at a given dislocation density. In addition, the longer lifetime of internal sources in larger micropillars increases the probability of generating new sources from an earlier source, preventing the dislocation-starved state seen in Figure 5.3. Thus, it is much harder to totally eliminate internal dislocations in larger samples than in smaller ones.

\subsection{Dislocation starvation (DS) model}

Since the rate of dislocation loss from free surfaces increases and the rate of dislocation multiplication decreases with decreasing sample size, there should be a critical size for FCC 


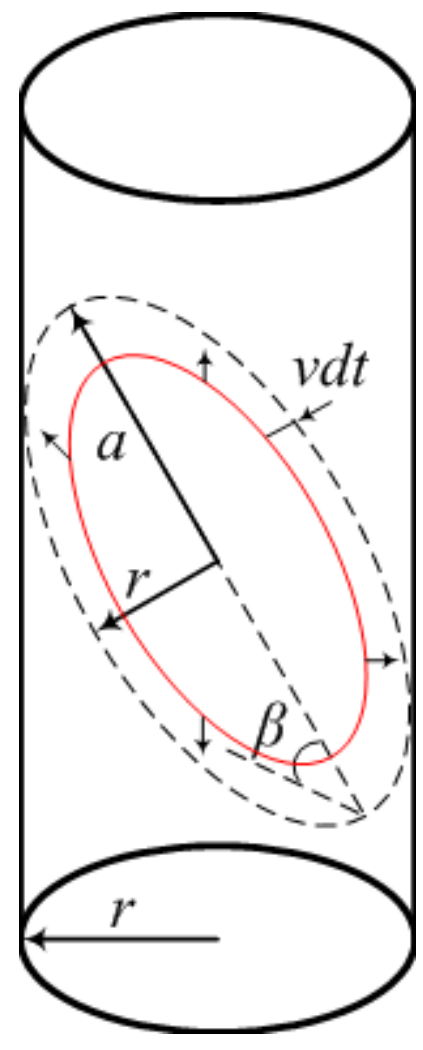

Figure 5.4 Schematic sketch of one dislocation loop in a finite cylindrical sample with the distance, $v d t$, from free surfaces.

single crystals below which the dislocation density should decline in the course of plastic deformation toward a dislocation-starved state. Here we present an analytical formulation for the Dislocation Starvation (DS) model, following an idea from ref. [19], that will enable us to predict that critical density.

Consider a cylindrical sample of radius $r$, with the primary slip plane oriented at an angle $\beta$ from the compressive axis as shown in Figure 5.4. The glide plane in this case is an ellipse with major axis $a=r / \cos \beta$ and the minor axis $r$. Assuming any dislocation within the distance $v d t$ from a free surface has a $50 \%$ chance of escaping from the surface, the dislocation loss rate is an inverse function of the sample size $r$, as follows 


$$
d \rho_{\text {loss }}=-\rho_{m o b}\left(\frac{1}{2} \frac{\pi(a+r) v d t}{\pi a r}\right)=-\rho_{m o b} \frac{\cos ^{2}(\beta / 2) v d t}{r}
$$

where $v$ is the dislocation velocity and $\rho_{m o b}$ is the mobile dislocation density. The dislocation losses from dislocation annihilation and locks have been ignored, since from our simulations they are small compared with surface losses. The dislocation multiplication rate is related to the mean free path, $L$ which is the distance traveled by a mobile dislocation before it is stored [42]

$$
d \rho_{m u l t}=\rho_{m o b} \frac{v d t}{L}
$$

The overall rate of total dislocation density, $\rho_{\text {total }}$, is the sum of dislocation loss and the multiplication rate, such that the total dislocation density evolves as:

$$
\rho_{\text {total }}=\rho_{o}+\frac{\left(1 / L-\cos ^{2}(\beta / 2) / r\right)}{b} \cdot \frac{\varepsilon_{p}}{M}
$$

where $\rho_{o}$ is the initial dislocation density, $b$ is the Burgers vector, $M$ is the Schmid factor and the plastic strain, $\varepsilon_{p}=\rho_{m o b} b v d t$. Based on eq. (5.4), samples can potentially arrive at a dislocation starvation state if the term related to the sample size, $\cos ^{2}\left(\frac{\beta}{2}\right) / r$, is smaller than the reciprocal of the mean free path. In addition, eq. (5.4) demonstrates that the dislocation density decreases faster with smaller sizes, which agrees with our DD simulation results.

From eq. (5.4), we can define a critical size, $D=2 r$, for dislocation starvation, below which the term, $\left(\frac{1}{L}-\cos ^{2}\left(\frac{\beta}{2}\right) / r\right)$ in eq. (5.4) is negative and the dislocation density will decrease with increasing strain leading to a dislocation-starved state at a given initial dislocation density. To estimate the critical size for dislocation starvation, we assumed that the mean free path of dislocations at small scales is approximated by $L \approx \rho^{-0.5}$ and $\beta=35^{\circ}$ for 
$<011>\{111\}$ slip systems. The critical size for dislocation starvation with a initial dislocation density $2.0 \times 10^{13} \mathrm{~m}^{-2}$ is about $400 \mathrm{~nm}$, and $1250 \mathrm{~nm}$ for initial dislocation density of $2.0 \times 10^{12}$ $\mathrm{m}^{-2}$. Recently, TEM analysis [14] revealed that the initial dislocation density in nanopillars, whether produced by a focused-ion beam or not, can easily reach on the order of $10^{14} \mathrm{~m}^{-2}$, suggesting that the mechanical response of nanoscale crystals is a stronger function of initial microstructure than of size regardless of fabrication method. In the case with dislocation density at $10^{14} \mathrm{~m}^{-2}$, the critical size for dislocation starvation is around $180 \mathrm{~nm}$, which is smaller than the sample sizes used in most previous experiments. This small size could explain why Shan et al. observed dislocation starvation only in their 150nm sample via in situ TEM [18] and why most experiments cannot find pristine samples with no internal dislocations after deformation [3, 10-11, 13-14, 17, 43-44]. Although this model considers the sample as a perfect single crystal without other kinds of defects, it still gives an instructive insight into the unconventional plasticity at nano- and micro-scales.

\subsection{Single-arm dislocation (SAD) model}

Unlike nanocompression tests, micropillars under compression are exposed to a nominally uniaxial stress and strain state. Thus, the observed size effects are likely to be more related to the stochastic behavior of dislocations than the gradient-induced storage of geometrically necessary dislocations [2]. In small volumes, a common dislocation source consists of a single dislocation arm with one end at a surface and the other at an internal pinning point. These single-arm sources can be formed either by the truncation of Frank-Read sources at free surfaces or directly generated in the initial dislocation structures. Guided by the observation of single-ended source operation in their 3-D DD simulations, Parthasarathy et al. developed the single-arm dislocation (SAD) model based on the observed dependence of the small-strain plastic response on the stochastic behavior of those sources [45]. Their goal was to calculate the increase and variation in the critical resolved shear 
stress by determining the stress required to activate the weakest single-arm dislocation source in a microcrystal when only one slip system is active. With these assumptions, the critical resolved shear stress at yielding can be calculated by adding the source activation stress to the lattice-friction stress and the back stress from the dislocation forest. They found that

$$
\tau=\tau_{o}+\frac{k \mu b}{\bar{\lambda}}+\alpha \mu b \sqrt{\rho}
$$

where $\tau_{o}$ is the lattice-friction stress, $k$ is the source-strength coefficient (with an average magnitude of $k=0.6$ [46]), $\alpha$ is the hardening coefficient ( 0.35 for FCC metals [47]), $\mu$ is the shear modulus, $b$ is the magnitude of the Burgers vector, $\rho$ is the dislocation density and $\bar{\lambda}$ is an effective source length calculated from the statistical model that is dependent on the sample dimensions and dislocation density [45].

In eq. (5.5), only the shear modulus and the Burgers vector show much variation for different FCC metals, while all other parameters are almost the same. Thus, it is useful to rearrange eq. (5.5) to make it independent of material parameters. Assuming the lattice-friction stress is negligible, we find

$$
\frac{\tau}{\mu b}=\frac{k}{\bar{\lambda}}+\alpha \sqrt{\rho}
$$

Since the right hand side of eq. (5.6) depends only on the effective source length $\bar{\lambda}$ and the dislocation density, we can use it to predict the yield stress of various FCC single crystals with different sample sizes and dislocation densities. In the original SAD model, the number of mobile dislocation sources was assumed to increase with the sample size at the sample yielding point. However, recent TEM observations [48-49] and 3-D DD simulation results $[22,29]$ indicated that only one, or at most a few, weakest sources determined the strength at the onset of plasticity. Thus, in the present study we determined the effective source length $\bar{\lambda}$, 


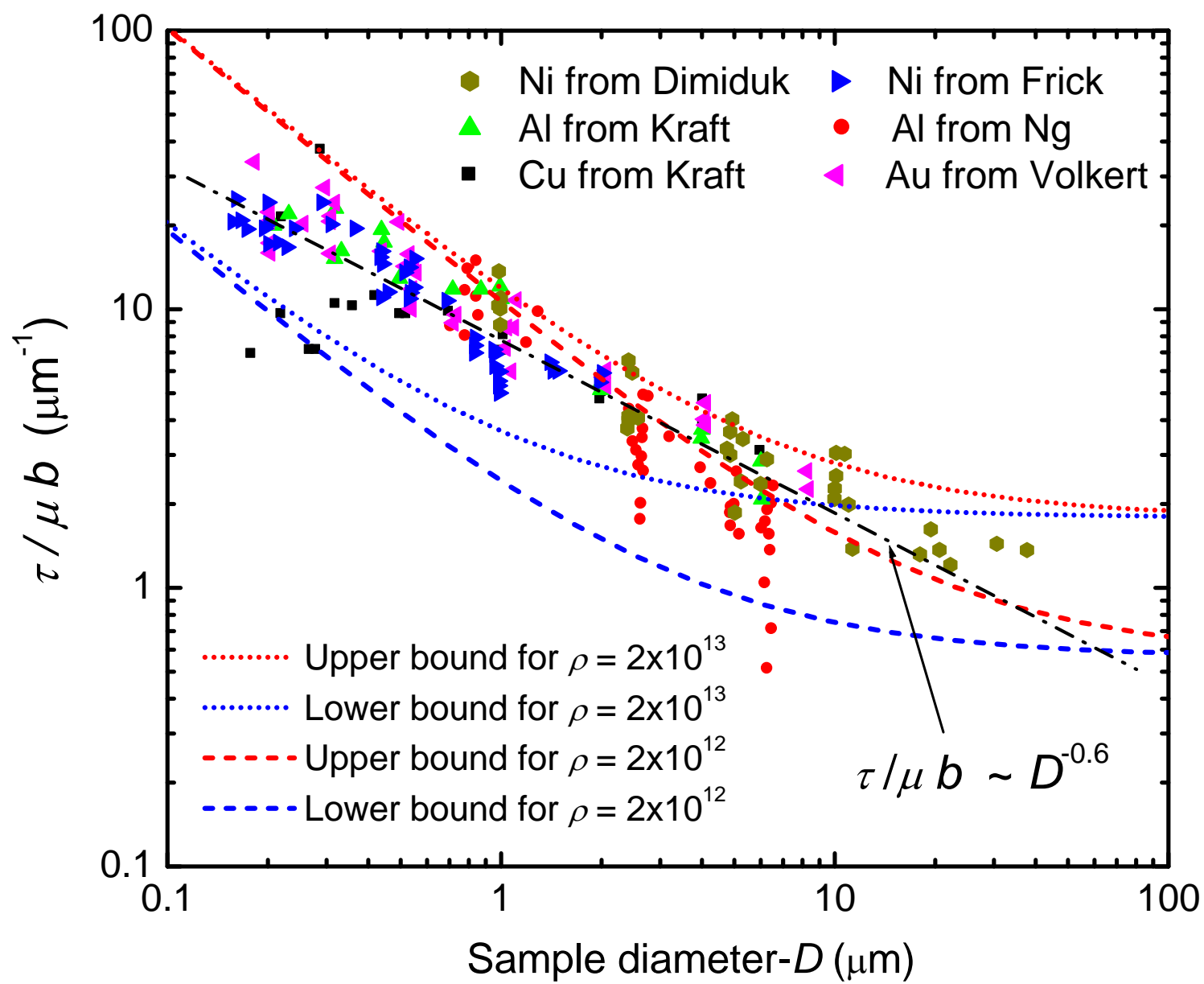

Figure 5.5 Comparison log-log plot of the general SAD model and microcompression results on various FCC single crystals.

from the statistical distribution of only one random single-arm source inside the sample. For validation, we present the data from microcompression tests from a number of FCC single crystals, $\mathrm{Ni}[2-3], \mathrm{Al}[9,11], \mathrm{Cu}[9]$ and $\mathrm{Au}[13]$, to compare with the predicted results from our version of the SAD model, where we assume limiting values of the dislocation density based on experimental observations $[2,6,10]$, with the high and low values being $2.0 \times 10^{12}$ $\mathrm{m}^{-2}$ and $2.0 \times 10^{13} \mathrm{~m}^{-2}$, respectively. All experimental data were taken at the onset of plasticity with a total strain less than $5 \%$. 
As shown in Figure 5.5, the normalized resolved shear stress for various FCC single crystals exhibits a similar size-dependent behavior with a scaling exponent of approximately 0.6. Furthermore, the general SAD model cannot only reasonably model the increase of yield strength with decreasing sample sizes, but also the statistical variation of the strength at small scales. The upper and lower bounds in Figure 5.5 were evaluated from the the standard deviations of the effective source length by the statistical model in ref. [45]. It is interesting to see that some of the sample sizes in Figure 5.5 are already below the critical size for dislocation starvation calculated in previous section, such as the sizes smaller than $400 \mathrm{~nm}$ at density equal $2.0 \times 10^{13} \mathrm{~m}^{-2}$. However, the SAD model still seems to capture the yield strength of these samples, perhaps because the process of thoroughly removing internal sources takes a long time at these small strain levels, even in $100 \mathrm{~nm}$ samples [49]. Thus the initial plastic deformation in these samples results from the motion of internal dislocation sources before they have been driven out of the sample.

\subsection{Dislocation interactions causing hardening at small scales}

The SAD model is designed to estimate the stress at the onset of yield and thus cannot explain the intermittency in plastic flow and discrete load increases in the post-yielding region, as observed in the microcompression tests [2-11, 13]. In section 3.1, we have shown that the strength increase in the post-yielding region can be caused by starvation hardening, i.e., mobile sources escaping from free surfaces. In this section, we will use DD simulations to investigate another type of hardening that arises from dislocation interactions during plastic flow. To that end, we extended our simulations to include realistic initial dislocation distributions.

We began our simulations with a cubic cell with periodic boundary conditions that was slightly deformed and unloaded, mimicking the deformation of a bulk single crystal. A cylinder was then cut out of the bulk sample with the aspect ratio of $D: H=1: 3$, where $D$ 


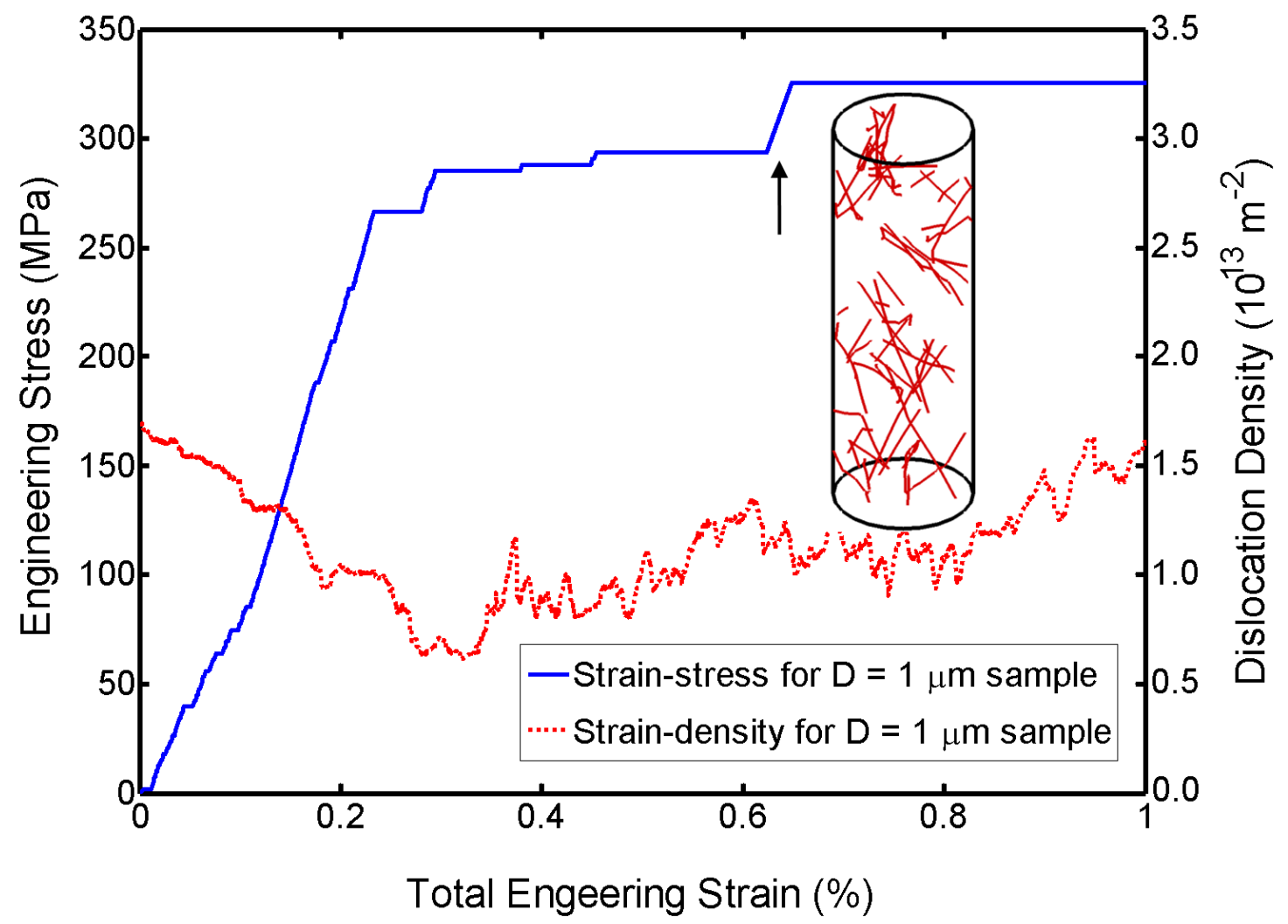

Figure 5.6 Stress-strain and density-strain curves obtained from simulations on the sample with $D$ $=1.0 \mu \mathrm{m}$.

and $H$ denote the diameter and height of the micropillar, respectively. The dislocation microstructures in the pillars were then relaxed under the influence of image and interaction forces until the initial dislocation structures reached a metastable configuration, as shown in the inset in Figure 5.6. The dislocation density after relaxation is around $1.7 \times 10^{13} \mathrm{~m}^{-2}$, which is consistent with conditions observed in experiments [17]. This experimental-like dislocation structure was then used as the initial configuration for our subsequent simulations. Further information about the simulation procedures can be found in reference [29].

Figure 5.6 shows the strain-stress curve for a $1.0 \mu \mathrm{m}$ diameter micropillar under compression along with the corresponding strain-dislocation density curve. One may observe in Figure 5.6 that a certain amount of "microplasticity" in the pillar was generated at low 
loads. This early-stage plasticity is often the result of weakly-entangled or surface dislocations being driven out of the system [29]. Owing to the escape of these dislocations, the dislocation density will decrease in the early stages as reflected by the strain-density curve in Figure 5.6. In addition, we see discrete strain bursts separated by nearly elastic loading after yielding, in agreement with experimental observations [2-11, 13]. This nearly elastic increase of load between each strain burst results from the activated sources being trapped by dislocation reactions and is called "exhaustion hardening." Figure 5.7 illustrates different dislocation configurations before and after exhaustion hardening, corresponding to the load increase in Figure 5.6 marked by an arrow. At the beginning of plastic flow, two single-arm spiral sources $S 1$ and $S 2$ cyclically sweep in their slip planes as shown in Figure 5.7a, producing continuous plastic flow. After the dislocations moved close to each other, a dislocation junction was formed that caused the cessation of plastic flow in the sample (Fig $7 \mathrm{~b}$ and $\mathrm{c})$. For plasticity to commence, additional dislocations had to be activated, which
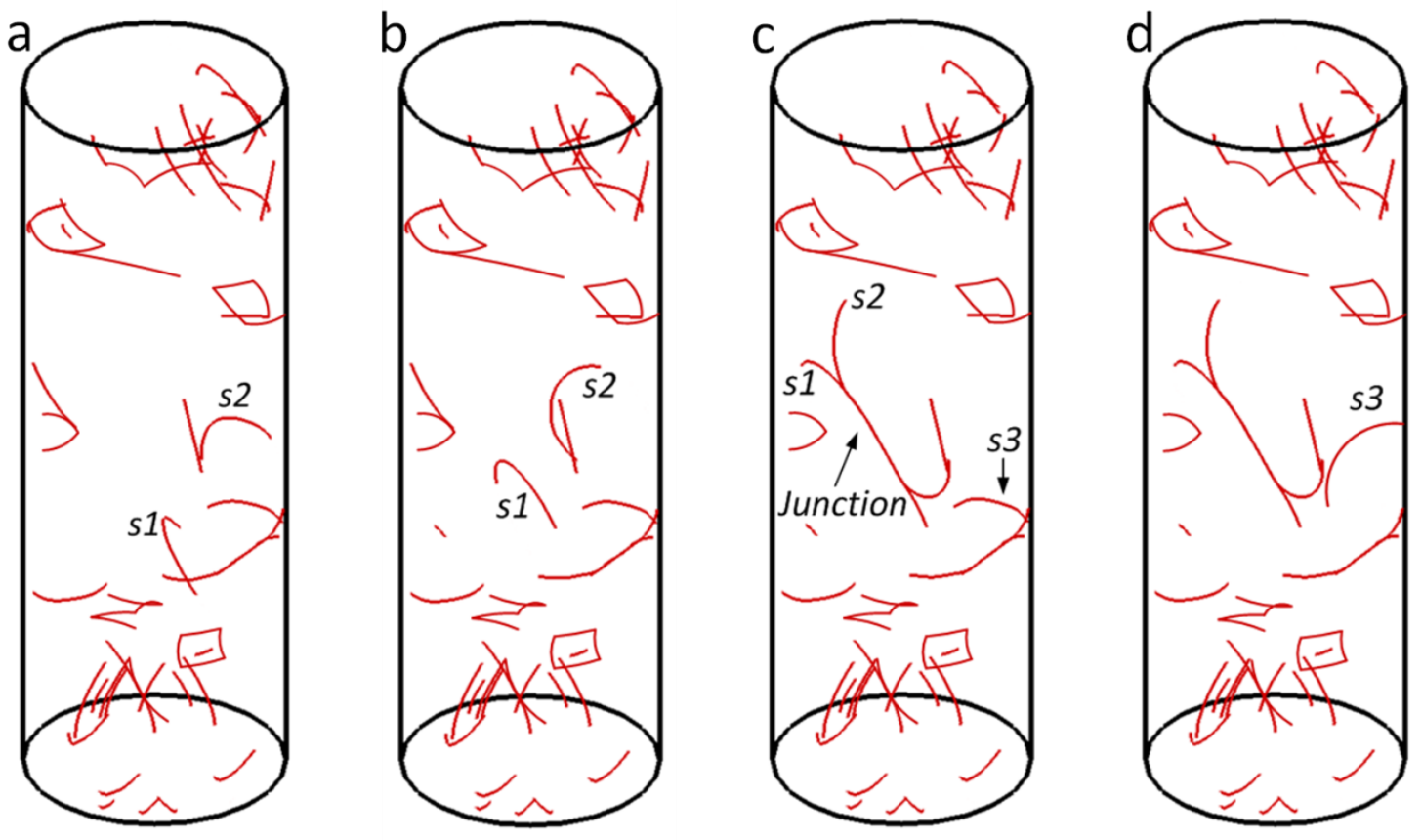

Figure 5.7 Plot of dislocation configurations before and after hardening caused by dislocation interactions. 
required the application of a significantly higher stress. After the applied stress increased to a sufficiently high level, S3 was activated (in Fig 7d), generating plastic deformation. To keep the same overall strain rate (a result of the experimental loading conditions assumed in this simulation), the applied stress stopped increasing, leading to a constant-stress flow in the stress-strain curve arising from the continuous operation of this new source.

The whole process in Figure 5.7 illustrates how multiplication of internal dislocation sources causes plastic flow in micro samples and also how dislocation interactions lead to intermittent plastic flow. When sample sizes decrease to the micro and submicron range, only one, or at most a few, mobile sources can carry all the plastic strain under loading. Thus, dislocation interactions with the mobile source will cause distinct changes in the plastic behavior of samples, resulting in highly jerky flow as manifest in stress-strain curves. In bulk samples, various dislocation interactions, such as dipole and junction formation, also occur in a similar way as in the micro samples and induce hardening, which is usually called "forest hardening". However, owing to the large number of mobile dislocation sources available for the plastic flow, the stress-strain curves of macro samples are much smoother than those of micro samples. It is worth noting that exhaustion hardening is always associated with plastic flow induced by the multiplication of internal dislocation sources, while starvation hardening is usually followed by the nucleation of surface dislocations.

\subsection{Implications for plasticity at small scales}

Combining our modeling and simulation results with dislocation structures observed in experiments [14, 17-18, 48-49], a physically based plastic deformation map for FCC single crystals at small scales has been presented in Figure 5.8, which divides the plasticity into three zones that depend on the sample size. The position of the zone boundaries are the

critical sizes for dislocation starvation as evaluated from the dislocation starvation model in section 3.2. 


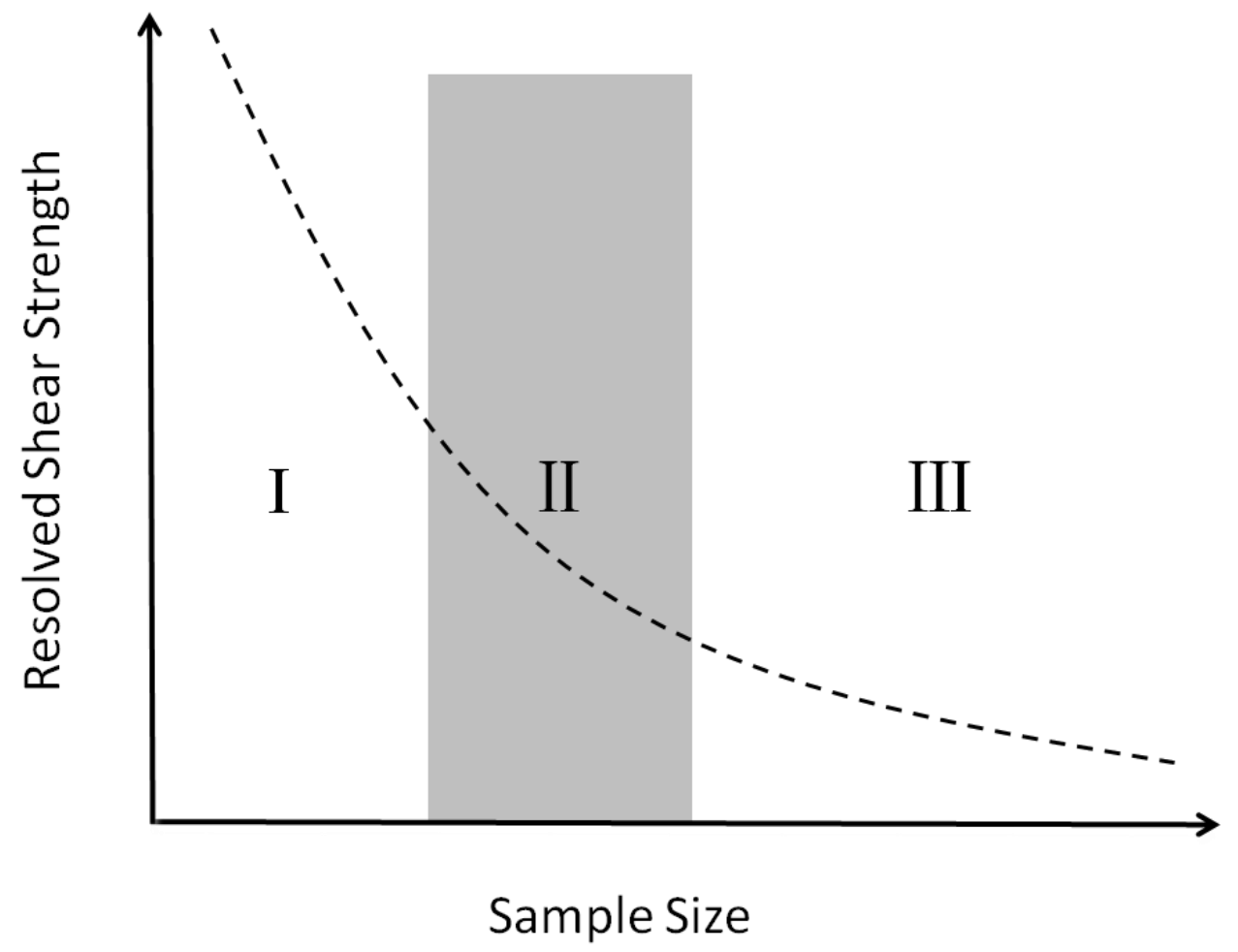

Figure 5.8 Complex deformation mechanism map for FCC single crystals: zone (I) nucleation of surface dislocations + starvation hardening, zone (II) nucleation/multiplication, depended on dislocation densities and structures, zone (III) multiplication of internal dislocations + exhaustion hardening.

In all three zones, the general single-arm dislocation (SAD) model can reasonably predict the strength at the onset of yielding induced by the action of a few internal dislocation sources. Beyond the initial plasticity, nucleation of surface dislocations or multiplication of internal dislocations are the two most probable mechanisms responsible for plastic flow in the post-yielding region. For samples located in zone I (very small samples), nucleation of surface dislocations will control the plastic deformation, with dislocation starvation being the dominant hardening mechanism, owing to the ease of dislocation sources escaping through the nearby surfaces. In contrast, in zone III (large systems), it is almost impossible to thoroughly eliminate internal sources through the free surfaces, so the multiplication of internal dislocations will carry all the plastic flow. Intermittent plasticity arises from the 
exhaustion hardening caused by dislocation interactions. In the transition zone II, both nucleation of surface dislocations and multiplication of internal dislocations are likely to occur, with a greater likelihood of nucleation-dominated plastic deformation for systems in proximity to zone I and a greater likelihood of multiplication-dominated plastic deformation for those near zone III. We note that the boundaries between these zones will likely be highly sensitive to the dislocation density and internal dislocation structures. For example, with increasing initial dislocation density, the position of zone II will shift to small sample size, which implies that the critical size for dislocation starvation should decrease correspondingly.

We can clearly see that the plastic deformation of FCC single crystals at small scales is not only size-dependent but is also dislocation density-dependent. With increasing dislocation density and sample size, multiplication of internal dislocations will likely control the plastic flow. In contrast, dislocation starvation and nucleation of surface dislocations should dominate the plastic deformation with decreasing density and size. As the observed dislocation densities from most experiments are on the order of $10^{12} \mathrm{~m}^{-2}$ to $10^{13} \mathrm{~m}^{-2}$, we can estimate that zone I in the plastic deformation map ends at around $0.5 \mu \mathrm{m}$ and zone III starts at around $1.0 \mu \mathrm{m}$.

\subsection{Concluding remarks}

3-D DD simulations were employed to study the dynamic behavior of internal dislocation sources in micropillars of different sizes. From the simulation results, we identified the dominating plastic deformation mechanisms at small scales by combining our modeling results. We note that these mechanisms are consistent with the available experimental data. 
In confined volumes, image stresses alter the local resolved shear stresses on slip planes, resulting in an increase in the probability of cross-slip to form new internal sources. These naturally formed sources have shorter residence lifetimes in smaller samples under the influence of attractive image forces from the nearby surfaces.

The normalized critically resolved shear stress for a number of FCC single crystals exhibited a similar size-dependent behavior for all the materials. The generalized single-arm dislocation model can reasonably predict both the increase of yield strength with decreasing sample size, as well as the statistical variation of the strength at small scales.

The plastic deformation of FCC single crystals at small scales depends not only on sample size but also on the dislocation density. At nano-and micro-scales, there is a critical size for dislocation starvation, which strongly depends on the initial dislocation density. Below this critical size, the dislocation loss rate will exceed the multiplication rate and thus nucleation of surface dislocations and dislocation starvation hardening will likely dominate plastic deformation process. Otherwise, multiplication of internal dislocation sources should control the plastic flow with increasing strain. 


\section{References}

[1] Uchic MD, Dimiduk DM, Florando JN, Nix WD. Sample dimensions influence strength and crystal plasticity. Science 2004;305:986.

[2] Dimiduk DM, Uchic MD, Parthasarathy TA. Size-affected single-slip behavior of pure nickel microcrystals. Acta Materialia 2005;53:4065.

[3] Frick CP, Clark BG, Orso S, Schneider AS, Arzt E. Size effect on strength and strain hardening of small-scale 111 nickel compression pillars. Materials Science and Engineering a-Structural Materials Properties Microstructure and Processing 2008;489:319.

[4] Greer JR, Nix WD. Size dependence of mechanical properties of gold at the sub-micron scale. Applied Physics a-Materials Science \& Processing 2005;80:1625.

[5] Greer JR, Nix WD. Nanoscale gold pillars strengthened through dislocation starvation. Physical Review B 2006;73.

[6] Greer JR, Oliver WC, Nix WD. Size dependence of mechanical properties of gold at the micron scale in the absence of strain gradients. Acta Materialia 2005;53:1821.

[7] Kiener D, Motz C, Schoberl T, Jenko M, Dehm G. Determination of mechanical properties of copper at the micron scale. Advanced Engineering Materials 2006;8:1119.

[8] Kim J-Y, Greer JR. Tensile and compressive behavior of gold and molybdenum single crystals at the nano-scale. Acta Materialia 2009;57:5245.

[9] Kraft O, Volkert C. Size effects on deformation and fatigue of thin films and small structures. Presented at CAMTEC 2006:Cambridge.

[10] Ng KS, Ngan AHW. Stochastic nature of plasticity of aluminum micro-pillars. Acta Materialia 2008;56:1712.

[11] Ng KS, Ngan AHW. Stochastic theory for jerky deformation in small crystal volumes with pre-existing dislocations. Philosophical Magazine 2008;88:677.

[12] Brinckmann S, Kim JY, Greer JR. Fundamental differences in mechanical behavior between two types of crystals at the nanoscale. Physical Review Letters 2008;100.

[13] Volkert CA, Lilleodden ET. Size effects in the deformation of sub-micron Au columns. Philosophical Magazine 2006;86:5567.

[14] Jennings AT, Burek MJ, Greer JR. Microstructure versus Size: Mechanical Properties of Electroplated Single Crystalline Cu Nanopillars. Physical Review Letters 2010;104. 
[15] Gao H, Huang Y, Nix WD, Hutchinson JW. Mechanism-based strain gradient plasticity - I. Theory. Journal of the Mechanics and Physics of Solids 1999;47:1239.

[16] Nix WD, Gao HJ. Indentation size effects in crystalline materials: A law for strain gradient plasticity. Journal of the Mechanics and Physics of Solids 1998;46:411.

[17] Norfleet DM, Dimiduk DM, Polasik SJ, Uchic MD, Mills MJ. Dislocation structures and their relationship to strength in deformed nickel microcrystals. Acta Materialia 2008;56:2988.

[18] Shan ZW, Mishra RK, Asif SAS, Warren OL, Minor AM. Mechanical annealing and source-limited deformation in submicrometre-diameter Ni crystals. Nature Materials 2008;7:115.

[19] Greer JR. Bridging the gap between computational and experimental length scales: A review on nanoscale plasticity. Reviews on Advanced Materials Science 2006;13:59.

[20] Greer JR, Weinberger CR, Cai W. Comparing the strength of f.c.c. and b.c.c. sub-micrometer pillars: Compression experiments and dislocation dynamics simulations. Materials Science and Engineering a-Structural Materials Properties Microstructure and Processing 2008;493:21.

[21] El-Awady JA, Biner SB, Ghoniem NM. A self-consistent boundary element, parametric dislocation dynamics formulation of plastic flow in finite volumes. Journal of the Mechanics and Physics of Solids 2008;56:2019.

[22] El-Awady JA, Wen M, Ghoniem NM. The role of the weakest-link mechanism in controlling the plasticity of micropillars. Journal of the Mechanics and Physics of Solids 2009;57:32.

[23] Rao SI, Dimiduk DM, Parthasarathy TA, Uchic MD, Tang M, Woodward C. Athermal mechanisms of size-dependent crystal flow gleaned from three-dimensional discrete dislocation simulations. Acta Materialia 2008;56:3245.

[24] Senger J, Weygand D, Gumbsch P, Kraft O. Discrete dislocation simulations of the plasticity of micro-pillars under uniaxial loading. Scripta Materialia 2008;58:587.

[25] Tang H, Schwarz KW, Espinosa HD. Dislocation escape-related size effects in single-crystal micropillars under uniaxial compression. Acta Materialia 2007;55:1607.

[26] Weygand D, Poignant M, Gumbsch P, Kraft O. Three-dimensional dislocation dynamics simulation of the influence of sample size on the stress-strain behavior of fcc 
single-crystalline pillars. Materials Science and Engineering a-Structural Materials Properties Microstructure and Processing 2008;483:188.

[27] Tang H, Schwarz KW, Espinosa HD. Dislocation-source shutdown and the plastic behavior of single-crystal micropillars. Physical Review Letters 2008;100.

[28] Motz C, Weygand D, Senger J, Gumbsch P. Initial dislocation structures in 3-D discrete dislocation dynamics and their influence on microscale plasticity. Acta Materialia 2009;57:1744.

[29] Zhou C, Biner SB, LeSar R. Discrete dislocation dynamics simulations of plasticity at small scales. Acta Materialia 2010;58:1565.

[30] Ghoniem NM, Sun LZ. Fast-sum method for the elastic field off three-dimensional dislocation ensembles. Physical Review B 1999;60:128.

[31] Ghoniem NM, Tong SH, Sun LZ. Parametric dislocation dynamics: A thermodynamics-based approach to investigations of mesoscopic plastic deformation. Physical Review B 2000;61:913.

[32] Wang ZQ, Ghoniem N, Swaminarayan S, Lesar R. A parallel algorithm for 3D dislocation dynamics. Journal of Computational Physics 2006;219:608.

[33] Kubin LP, Canova G. THE MODELING OF DISLOCATION PATTERNS. Scripta Metallurgica Et Materialia 1992;27:957.

[34] Verdier M, Fivel M, Groma I. Mesoscopic scale simulation of dislocation dynamics in fcc metals: Principles and applications. Modelling and Simulation in Materials Science and Engineering 1998;6:755.

[35] Rao S, Parthasarathy TA, Woodward C. Atomistic simulation of cross-slip processes in model fcc structures. Philosophical Magazine a-Physics of Condensed Matter Structure Defects and Mechanical Properties 1999;79:1167.

[36] Starenchenko VA, Lychagin DV, Shaekhov RV, Kozlov ÉV. Action of test temperature on evolution of dislocation structure of nickel single crystals with the [001] compression axis. Russian Physics Journal 1999;42:653.

[37] Oh SH, Legros M, Kiener D, Dehm G. In situ observation of dislocation nucleation and escape in a submicrometre aluminium single crystal. Nature Materials 2009;8:95.

[38] Oh SH, Legros M, Kiener D, Gruber P, Dehm G. In situ TEM straining of single crystal $\mathrm{Au}$ films on polyimide: Change of deformation mechanisms at the nanoscale. Acta Materialia 2007;55:5558. 
[39] Murr LE. Interfacial Phenomena in Metals and Alloys Addison-Wesley Educational Publishers Inc 1975.

[40] Weinberger CR, Cai W. Surface-controlled dislocation multiplication in metal micropillars. Proceedings of the National Academy of Sciences of the United States of America 2008;105:14304.

[41] Madec R, Devincre B, Kubin L, Hoc T, Rodney D. The role of collinear interaction in dislocation-induced hardening. Science 2003;301:1879.

[42] Devincre B, Hoc T, Kubin L. Dislocation mean free paths and strain hardening of crystals. Science 2008;320:1745.

[43] Lee SW, Han SM, Nix WD. Uniaxial compression of fcc Au nanopillars on an $\mathrm{MgO}$ substrate: The effects of prestraining and annealing. Acta Materialia 2009;57:4404.

[44] Maaß R, Van Petegem S, Zimmermann J, Borca CN, Van Swygenhoven H. On the initial microstructure of metallic micropillars. Scripta Materialia 2008;59:471.

[45] Parthasarathy TA, Rao SI, Dimiduk DM, Uchic MD, Trinkle DR. Contribution to size effect of yield strength from the stochastics of dislocation source lengths in finite samples. Scripta Materialia 2007;56:313.

[46] Rao SI, Dimiduk DM, Tang M, Parthasarathy TA, Uchic MD, Woodward C. Estimating the strength of single-ended dislocation sources in micron-sized single crystals. Philosophical Magazine 2007;87:4777.

[47] Kubin L, Devincre B, Hoc T. Modeling dislocation storage rates and mean free paths in face-centered cubic crystals. Acta Materialia 2008;56:6040.

[48] Legros M. How microstructure imposes size effects on the strength of sub-micron Al fibers. Gordon Research Conferences on Thin Film \& Small Scale Mechanical Behavior, Waterville, Maine, USA; 2010., 2010.

[49] Kiener D, Minor A. Uniaxial quantitative in situ testing of fcc and bec metals in a TEM. Gordon Research Conferences on Thin Film \& Small Scale Mechanical Behavior, Waterville, Maine, USA; 2010., 2010. 


\section{CHAPTER 6}

\section{SIMULATIONS OF THE EFFECT OF SURFACE COATINGS ON PLASTICITY AT SMALL SCALES}

Small-scale metallic structures have been widely utilized in microelectronic circuits, optical and magnetic storage media, micro-electro-mechanical systems (MEMS) and so on, owing to their excellent mechanical, chemical, or electrical properties. Recently, size-dependent deformation properties of single crystals have attracted much attention in the materials science community, in part because these properties are closely related to the reliability of such structures in technical applications.

The sudden structural softening under compression arising from large strain bursts of small scale materials is a critical problem for engineering application of such metallic structures. Recently, Ng and Ngan [1] found that coating aluminum microcolumns with tungsten significantly increased the compressive strength and alleviated strain bursts compared with the free surface samples agreeing with Greer's experiment results on $\mathrm{Au}$ micropillars coated by $\mathrm{Al}_{3} \mathrm{O}_{2}$ [2]. In addition, subcells and band structures always formed in the coated samples which have seldom been observed in free surface samples. However, the remarkable increase in strength and strain hardening rate of micropillars by hard coatings could not be explained by the conventional rule of mixtures, which indicates the strength of mixtures should be equal to the sum of the strength of each component multiplying their volume fractions [1].

In this chapter, we employed 3-D DD simulations to examine the mechanical behavior and microstructure evolution in micropillars with a hard coating layer similar to samples studied experimentally [1]. The experiment-like initial dislocation structures containing FR sources, jogged dislocations, surface dislocations and spiral (single-armed) sources was 
introduced into our DD simulations to study plastic behavior of Ni single-crystal micropillars under compression. We compare the results from coated micropillars to those from free-surface micropillars to investigate how coatings affect the plastic deformation behavior in small volumes.

\subsection{Simulation procedures}

We employ the PDD method described in Chapter 2 to simulate the mechanical behavior of Ni single crystals under uniform compression in [001] direction. For the simulations in this work, the applied strain rate was equal to $200 \mathrm{~s}^{-1}$ with the experimental loading conditions described in Chapter 4, and the materials properties of nickel were used: shear modulus $\mu=$ $76 \mathrm{GPa}$, Poisson's ratio $v=0.31$, and lattice constant $\mathrm{a}=0.35 \mathrm{~nm}$. Finally, a sophisticated thermally-activated cross-slip model developed by Kubin and co-workers [3-4] was adopted in our DD simulations with Monte Carlo sampling to determine if cross-slip is activated or not. Detailed procedures can be found in ref [5].

At beginning, a larger cubic cell was generated with periodic boundary conditions and a size $3 \times 3 \times 3 \mu \mathrm{m}^{3}$ containing a set of FR sources with an initial density equal to $2.0 \times 10^{12} \mathrm{~m}^{-2}$. The FR sources (straight dislocation segments pinned at both ends) were randomly set on all twelve $<011>\{111\}$ slip systems with random lengths. After compression in the [111] direction to a dislocation density of about $2.5 \times 10^{13} \mathrm{~m}^{-2}$, the "bulk" cubic sample was unloaded and cylinders of various sizes (representing micropillars) were cut out of the bulk sample along the [001] direction with the aspect ratio of $D: H=1: 2$, where $D$ and $H$ denote the diameter and height of micropillars, respectively. Subsequently, the deformed dislocation microstructures were relaxed under the influence of image and interaction forces. The dislocation densities after relaxation were all in the range of 1.0 to $2.0 \times 10^{13} \mathrm{~m}^{-2}$ and consistent with conditions observed in experiments [6]. In the present study, samples with diameters $D=0.5$ and $1.0 \mu \mathrm{m}$ were compressed in [001] direction with coated and free 
surfaces. In agreement with the experimental observations [1], the hard coating layer was considered as an impenetrable obstacle for dislocations, while the free surface could annihilate dislocations and generate image force attracting dislocations, which play a significant role in mechanical behavior of uncoated mciro- and nano- samples [7]. The free surfaces were modeled using the boundary element method (BEM) described in Chapter 3.

\subsection{Effect of trapping dislocations}

In Figure 6.1, the engineering stress-strain relationships of two micropillar sizes $D=0.5$ and $1.0 \mu \mathrm{m}$ are shown. The size-dependent behavior, in which smaller samples have higher strength, is observed in both coated and free-surface samples. When the identical initial dislocation configurations used, the flow stress of coated samples is approximated $110 \%$

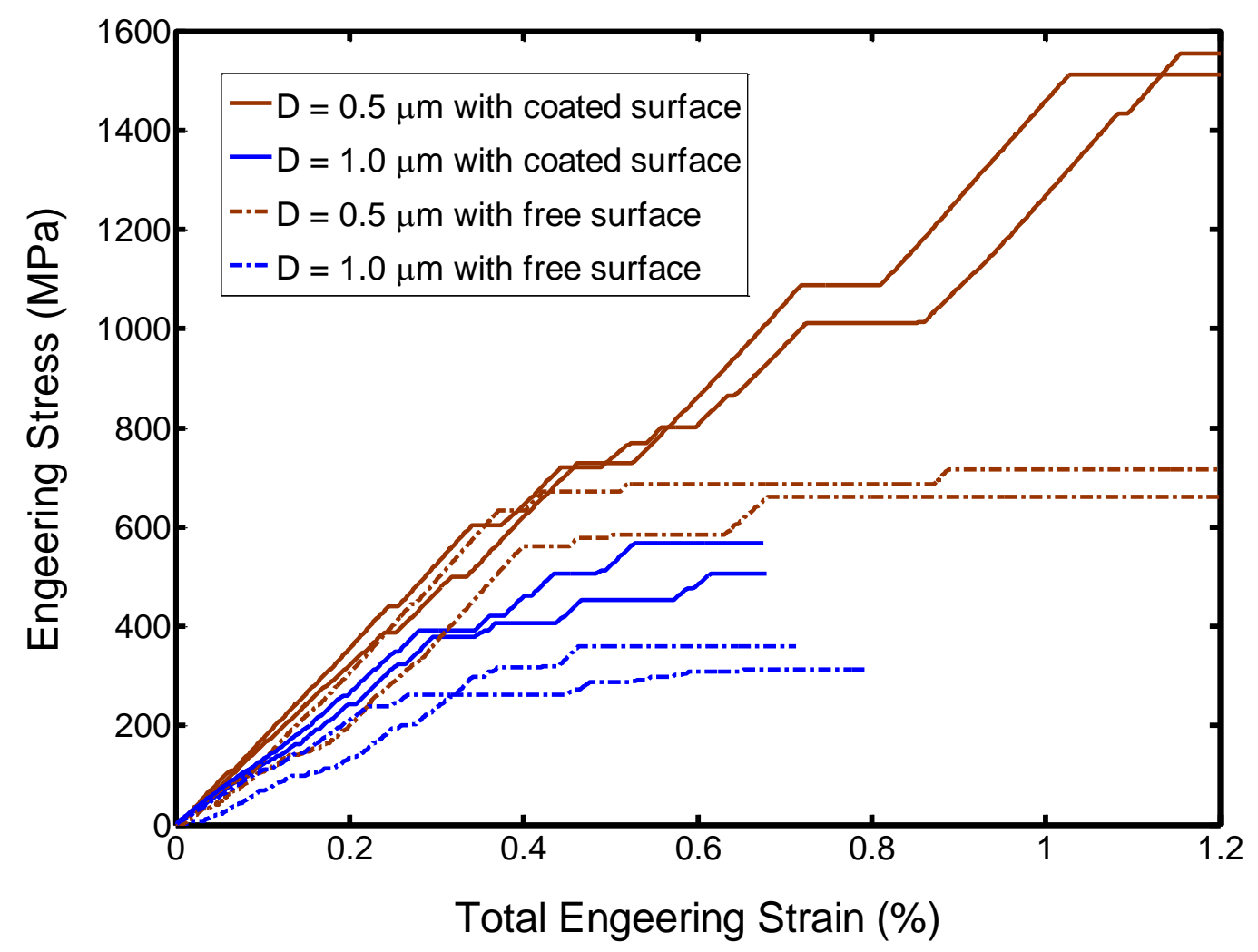

Figure 6.1 Stress-strain curves for both coated and uncoated samples 
higher than that of the free-surface samples with $D=0.5 \mu \mathrm{m}$ and $60 \%$ higher with $D=1.0$ $\mu \mathrm{m}$. In the coated samples, stress-strain curves exhibited larger stain hardening rates than those did samples with free surfaces, and the magnitude of strain bursts was decreased to a large degree, which agrees well with experiment results that coated samples demonstrated a significant increase in flow stress and the strain-hardening rate [1-2].

Figure 6.2a shows the comparison of the strain-stress curves and the corresponding variation of the dislocation density for a typical example of a coated and a free-surface micropillar with diameter $D=1.0 \mu \mathrm{m}$. The identical initial dislocation structure is shown in Figure 6.2b. The coated sample exhibited not only higher flow stress, but also a higher hardening rate i.e., at the same stress level, the total strain is smaller than that in free-surface sample. The large amount of "microplasticity", plastic strain at low loads, in samples with free surfaces is the result of the weakly-entangled dislocations from within the sample being driven out free surface [5]. In the coated sample, however, dislocations are blocked by the coated layer and stored near the interfaces, which induces a strong back stress on that activated dislocations later in time. Thus, the plastic deformation induced by the movement of dislocations was smaller in the coated sample at low loads and it showed steeper slope of strain-stress curves before yielding. This large difference in dislocation behavior in the two cases was also reflected in the corresponding strain-dislocation density curves in Figure 6.2a. For the free-surface case, dislocation density decreased at the early stage of deformation because of the mobile dislocations leaving the sample and then increased slightly with strain as cross-slip was activated, which was also demonstrated in the experiment observations [8]. Although the dislocation starvation model seems to be valid at small diameters (about $150 \mathrm{~nm}$ ) [9], because of the sizes of our samples larger than 300nm, an absolute dislocation-free state is almost impossible in our simulations. Dislocations were always observed in the experiments for samples with diameter, $D>300 \mathrm{~nm}[10]$. In coated samples, early activated dislocations could not leave through the surface and were stored near the 


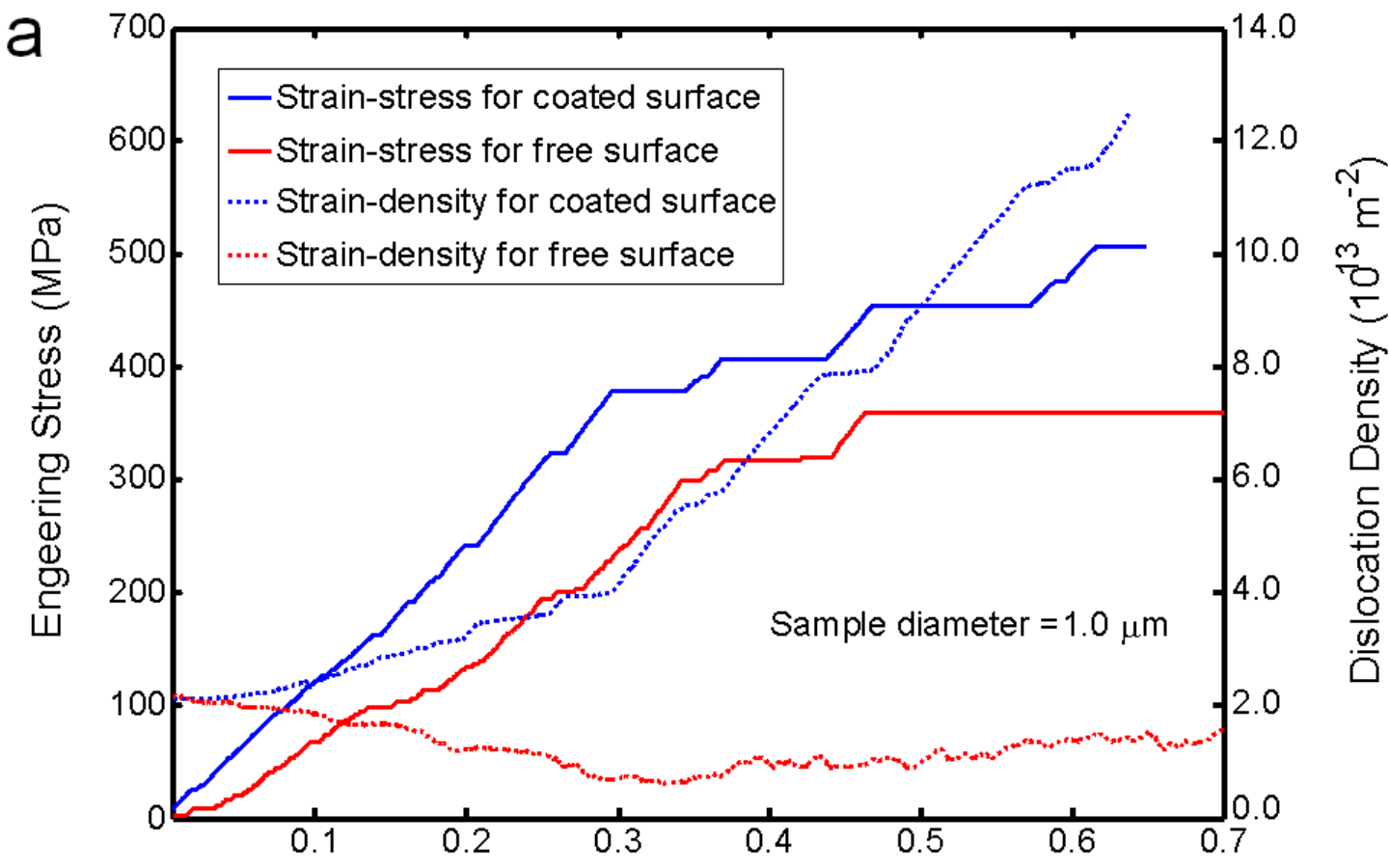

Total Engineering Strain (\%)
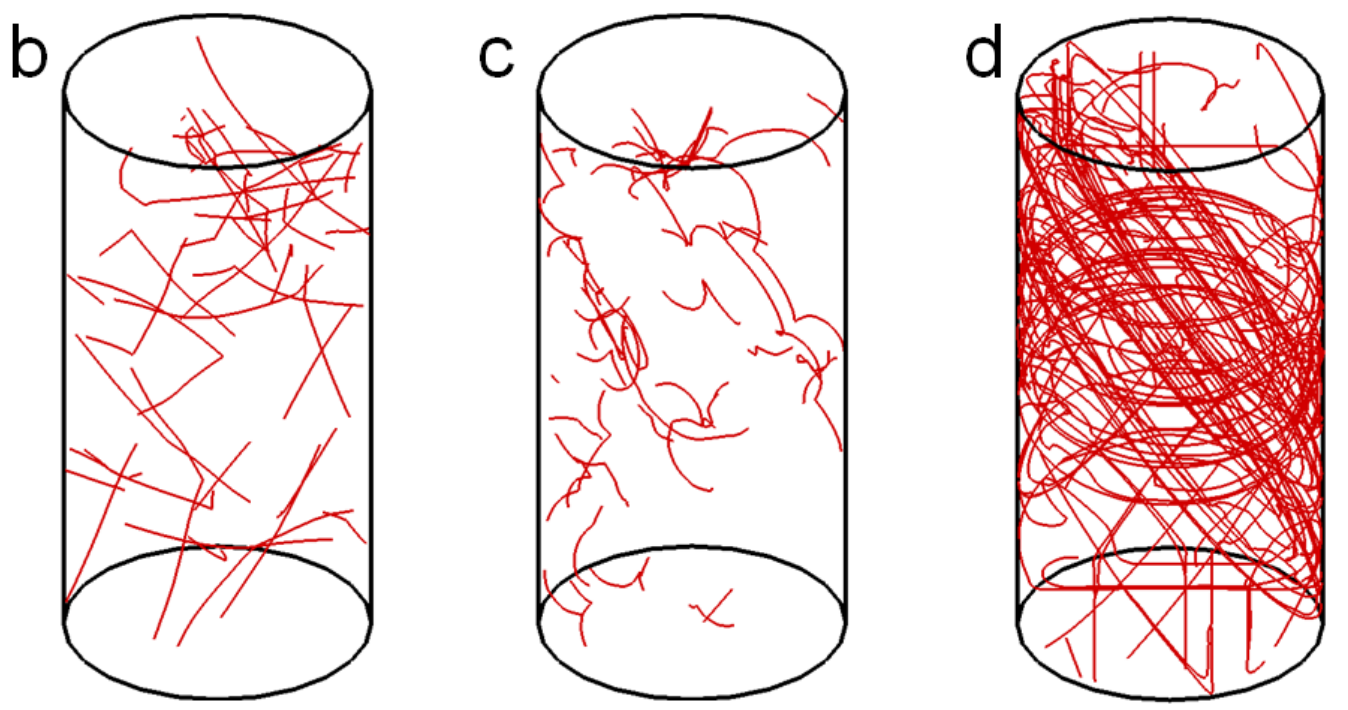

Figure 6.2 (a) Stress-strain and dislocation density-strain curves with diameter $D=1.0 \mu \mathrm{m}$, (b) initial dislocation structure, (c) dislocation structure in free-surface sample at $0.6 \%$ strain and (d) dislocation structure in coated sample at $0.6 \%$ strain. 
interface, so total dislocation density gradually increased. At some critical applied stress, which was larger than the flow stress of free-surface sample, there was a sharp increase in dislocation density, indicating that a large number of dislocation had been activated creating more mobile sources for plastic deformation. However, the higher the number of activated sources, the greater the dislocation densities near the interface, which in turn induces addition back stress that inhibits further dislocation nucleation. Thus, the large strain bursts in free surface samples were not present in coated samples, but were replaced by smaller strain bursts separated by elastic hardening stages (see Figure 6.1). The dislocation structures of both the uncoated and coated samples at a strain level of $0.6 \%$ are plotted in Figure $6.2 \mathrm{c}$ and $\mathrm{d}$, respectively. Compared with the initial configuration in Figure $6.2 \mathrm{~b}$, the dislocation density was relatively unchanged in the samples with free surfaces, but the dislocation lines have changed from being relatively long and straight into being short and jogged, which results from dislocation reactions and cross-slip. In contrast, the density in the coated sample increases to around five times the initial density at $0.6 \%$ strain and showed a large number of dislocations pile-ups near interfaces. Note that banded structures were gradually formed as the strain increased.

\subsection{Banded structures formed by cross-slip}

To investigate the effect of cross-slip on the evolution of dislocation structures and the mechanical behavior of coated samples, an additional sample with $D=1.0 \mu \mathrm{m}$ was cut from the undeformed cube shown in Figure 6.3b, such that Frank-Read and spiral sources were initially present (with an initial density of $1.8 \times 10^{12} \mathrm{~m}^{-2}$ ). This sample was put under load with and without cross-slip enabled. Figure 6.3a shows the comparison of the stress and dislocation density evolution for these two cases. The non-cross-slip case exhibited essentially hardening, except for several insignificant strain jumps corresponding to the discrete increase of dislocation density in the density-strain curve. The dislocation structure 

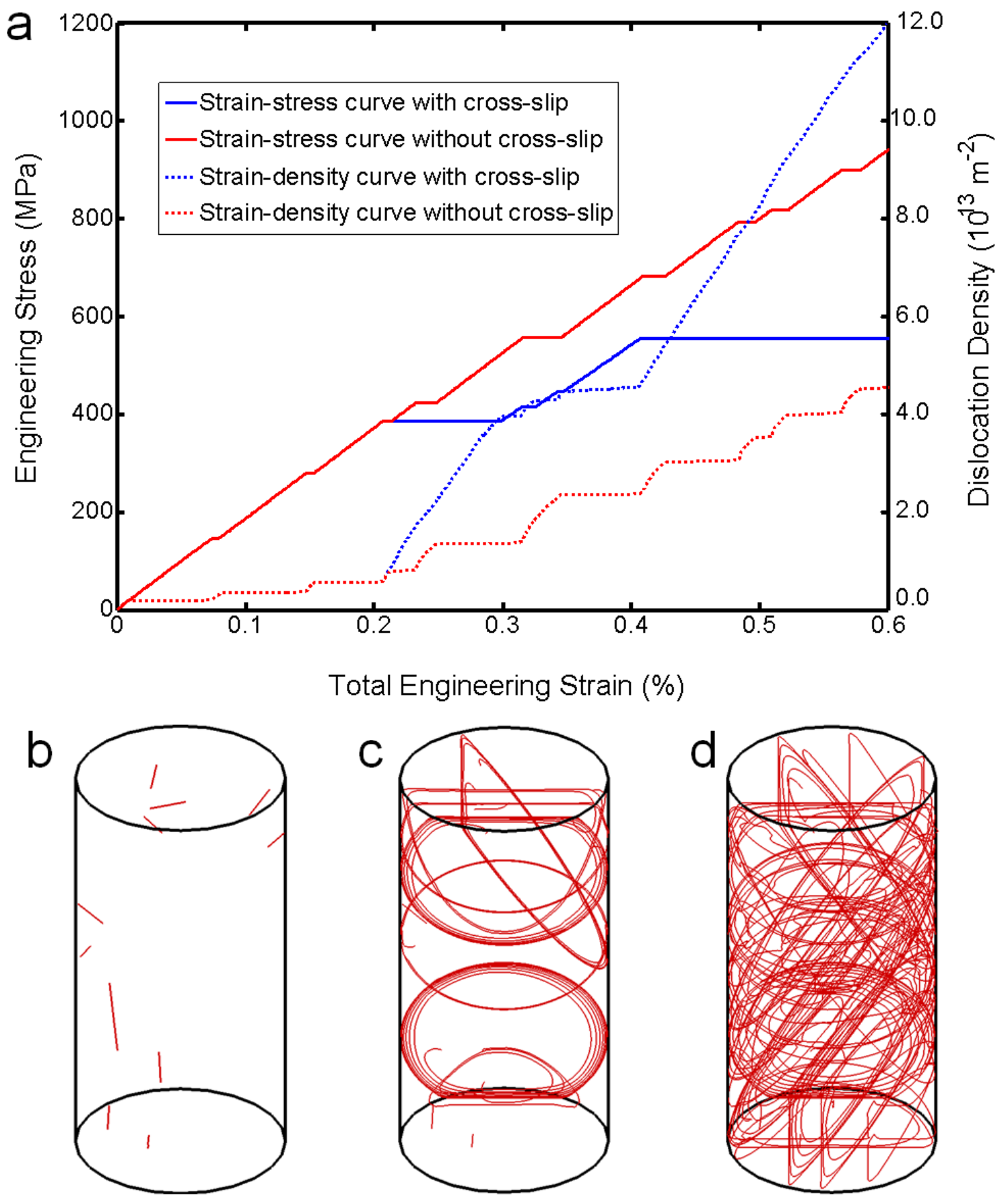

Figure 6.3 (a) Stress-strain and dislocation density-strain curves with diameter $D=1.0 \mu \mathrm{m}$, (b) initial dislocation structure, (c) dislocation structure without cross-slip at $0.6 \%$ strain and (d) dislocation structure with cross-slip at $0.6 \%$ strain. 
for non-cross-slip case at $0.6 \%$ stain is plotted in Figure $6.3 \mathrm{c}$, which indicated dislocation sources were just nucleated on several slip planes. Since fewer sources available in the non-cross-slip sample, each source had to repeatedly operate on the same slip plane to sustain the applied strain. This repeated process required much larger external loading to overcome the high back stress generated by previous pile-up dislocations than nucleating new sources on slip planes without pile-up dislocations, so non-cross-slip sample exhibited smaller strain bursts and higher strength. In contrast, cross-slip enables screw dislocations under a strong back stress to escape from the original slip plane to the cross-slip plane, generating new dislocation sources that led to more plastic deformation. As shown in Figure 6.3a, the stress and density curves of the cross-slip and non-cross-slip cases were very similar below about $0.2 \%$ strain. After that, there is a sharp increase of dislocations in the cross-slip-enabled sample reflected in strain-stress curve by strain bursts that indicates new dislocation sources were generated. The corresponding dislocation structure for the cross-slip case at $0.6 \%$ stain is shown in Figure 6.3d, from which we could see that more slip systems have been activated than of in the non-cross-slip case in Figure 6.3c. Banded structures were formed, in which double-cross slip plays an important role. Figure 6.4 illustrated one typical example of double-cross slip in the coated sample. In Figure 6.4a, the dislocation source $L 1$ with slip system $\frac{1}{2}[101](\overline{1} 11)$ approached the interface, experiencing a high repulsive stress from previous piled-up dislocations on the same slip plane. Under the combination of applied stress and dislocation interaction stress, the screw segment $S 1$ of dislocation $L 1$ cross-slipped on the plane $(\overline{1} 11)$, to escape the original slip plane $(\overline{1} 11)$, as shown in Figure $6.4 \mathrm{~b}$. S1 continued bowing out under the applied stress in Figure 6.4c. Finally, double-cross slip produced new dislocation sources in the slip plane parallel to the original one, as shown in Figure $6.4 \mathrm{~d}$. The whole procedure was continuously repeated generating large number of dislocation pile-ups on the cross-slipped planes as well as in the planes parallel to the original ones, forming banded structures and subcells as observed in experiments [1]. Thus, cross-slip 

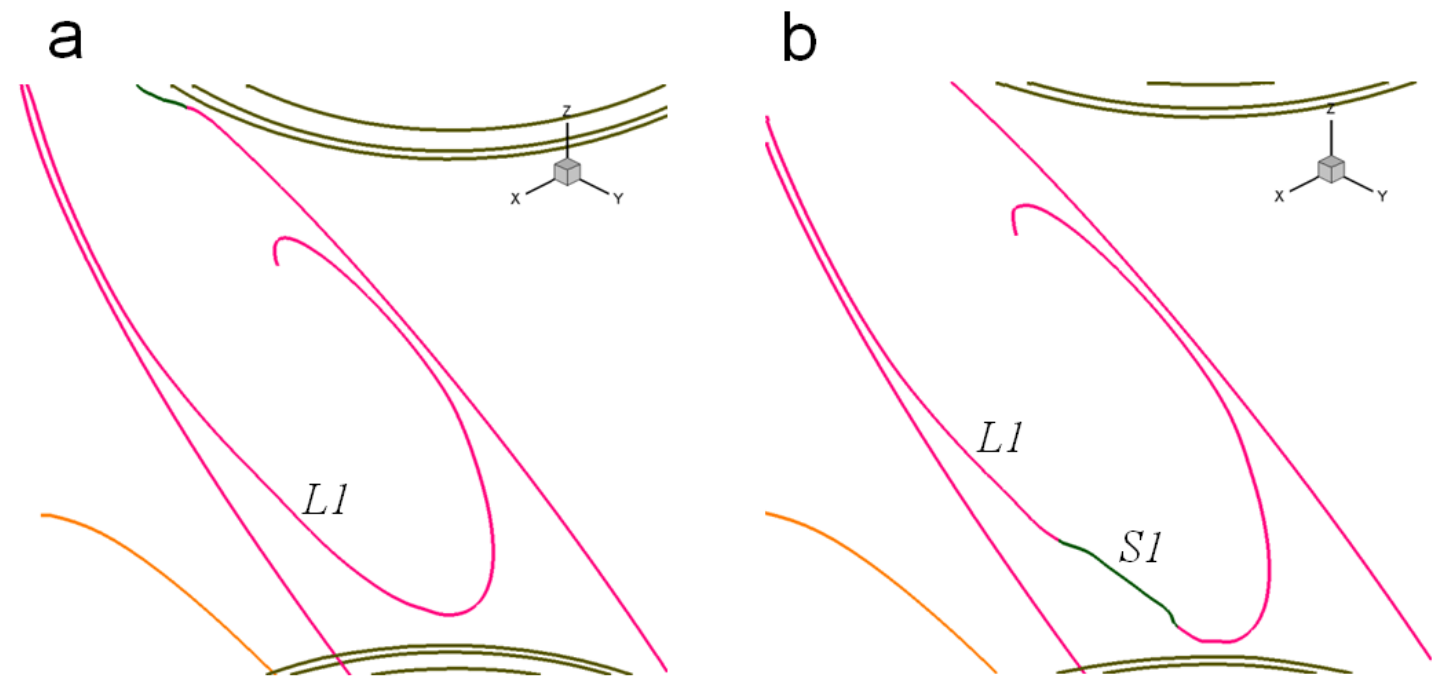

\section{C}
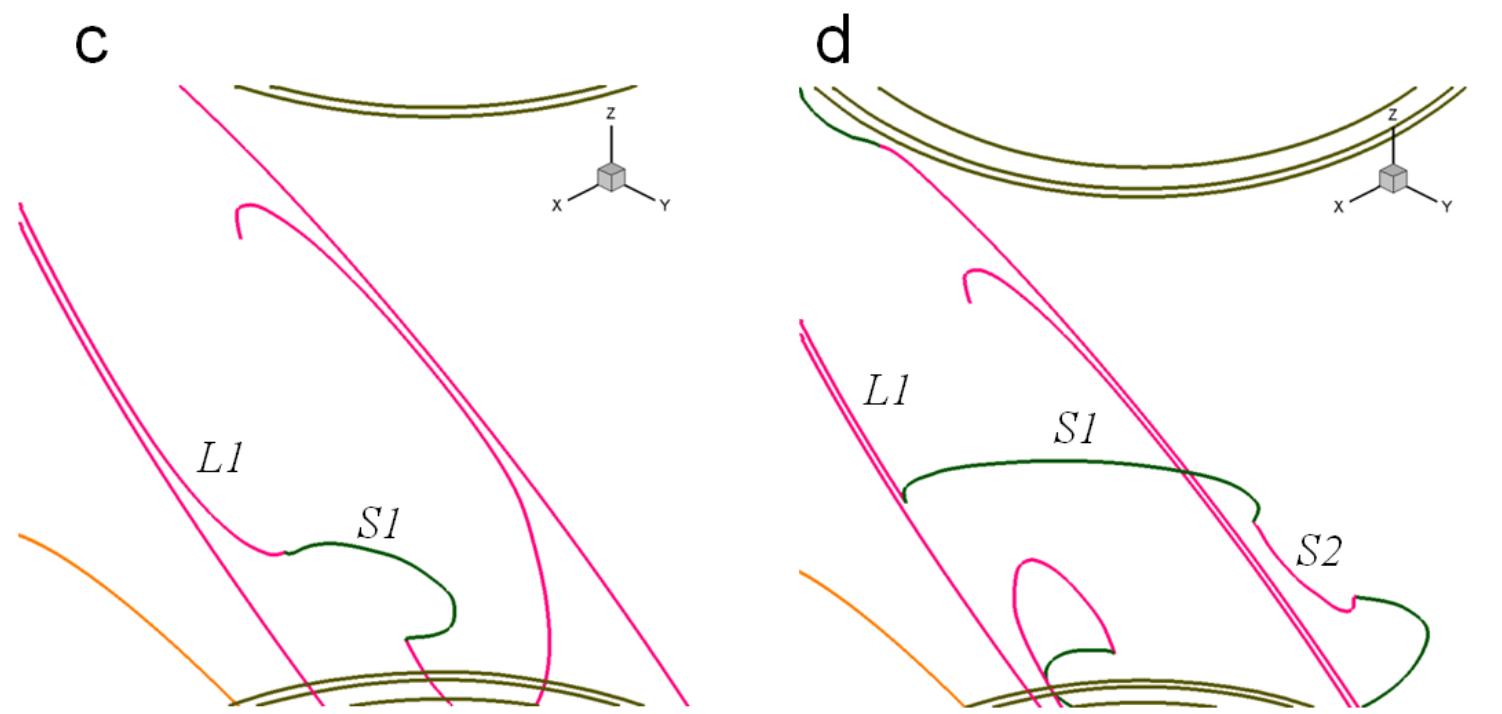

Figure 6.4 Plot of double cross-slip in coated sample: red line with $1 / 2[101](\overline{1} 11)$ and green line with $1 / 2[101](\overline{1} \overline{1} 1)$, see details in text.

seems to be the primary mechanism in producing additional dislocation sources during the plastic deformation of small coated samples.

\subsection{Concluding remarks}


Our simulations offer an explanation for the significant increase in compressive strength and formation of band structures in coated micropillars, demonstrating a fundamentally different strengthening mechanism in coated micropillars than in samples with free surfaces. Normally, in free-surface samples, image forces attract dislocations to the surface, where they exit, leaving a relative clear free path for dislocations that are activated later. In the coated samples, dislocations are blocked from leaving the sample, leading to dislocation pile-ups that induce a strong back stress on the later-activated sources, inhibiting further dislocation nucleation. The more dislocation pile-ups, the higher back stresses. Thus, the coated samples exhibited a higher strain-hardening rate, smaller strain bursts and greater flow stresses than those in samples with free surface. In addition, cross slip activated in coated samples enable screw dislocations to escape their original slip plane, generating more mobile dislocation sources for plastic deformation, and enabling the formation of banded structures and subcells. 


\section{References}

[1] $\mathrm{Ng} \mathrm{KS}$, Ngan AHW. Effects of trapping dislocations within small crystals on their deformation behavior. Acta Materialia 2009;57:4902.

[2] Greer JR. Effective use of Focused Ion Beam (FIB) in investigating fundamental mechanical properties of metals at the sub-micron scale. 2006 MRS Fall Meeting, November 27, 2006 - December 1, 2006, vol. 983. Boston, MA, United states: Materials Research Society, 2007. p.69.

[3] Kubin LP, Canova G. The modeling of dislocation patterns. Scripta Metallurgica Et Materialia 1992;27:957.

[4] Verdier M, Fivel M, Groma I. Mesoscopic scale simulation of dislocation dynamics in fcc metals: Principles and applications. Model. Simul. Mater. Sci. Eng. 1998;6:755.

[5] Zhou C, Biner SB, LeSar R. Discrete dislocation dynamics simulations of plasticity at small scales. Acta Materialia 2010;58:1565.

[6] Norfleet DM, Dimiduk DM, Polasik SJ, Uchic MD, Mills MJ. Dislocation structures and their relationship to strength in deformed nickel microcrystals. Acta Materialia 2008;56:2988.

[7] Weinberger CR, Cai W. Surface-controlled dislocation multiplication in metal micropillars. Proc. Natl. Acad. Sci. U. S. A. 2008;105:14304.

[8] Ng KS, Ngan AHW. Stochastic nature of plasticity of aluminum micro-pillars. Acta Materialia 2008;56:1712.

[9] Shan ZW, Mishra RK, Asif SAS, Warren OL, Minor AM. Mechanical annealing and source-limited deformation in submicrometre-diameter $\mathrm{Ni}$ crystals. Nat. Mater. 2008;7:115.

[10] Lee SW, Han SM, Nix WD. Uniaxial compression of fcc Au nanopillars on an MgO substrate: The effects of prestraining and annealing. Acta Materialia 2009;57:4404. 


\section{CHAPTER 7}

\section{DISLOCATION DYNAMICS SIMULATIONS OF PLASTICITY IN POLYCRYSTALLINE THIN FILMS}

In this study, 3-D DD simulations considering both cross-slip of dislocations and stress relaxation at grain boundaries have been used to investigate the size-dependent plasticity of polycrystalline thin films. According to our simulation results, we relate the plastic deformation of polycrystalline thin films to such quantities as dislocation density, grain size and thin film thickness, and finally developed a spiral source model to predict the plastic behavior of thin films.

\subsection{Simulation procedures}

The DD simulations are performed with the code UCLA-Microplasticity. Details on the methods used in this code were described in Refs [1-3]. For the simulations in this work, the materials properties of $\mathrm{Cu}$ are used: shear modulus $\mu=50 \mathrm{GPa}$, Poisson's ratio $v=0.34$, and lattice constant $\mathrm{a}=0.36 \mathrm{~nm}$. The dislocation mobility is taken to be $10^{-4} \mathrm{~Pa}^{-1} \mathrm{~s}^{-1}$ in the calculations [4].

Cross-slip of dislocations is important for the plastic deformation of crystal materials, even at small scales [5-8]. The moving dislocation leaves its habit planes and propagates on the cross-slip plane to generate new sources for the following plastic deformation or enable oppositely signed screw dislocations on different planes to annihilate each other. In our simulations, a sophisticated thermally-activated cross-slip model developed by Kubin and co-workers [9-10] was adopted in our DD simulations to study the evolution of microstructures on in thin films, and a Monte Carlo method was used to check whether cross-slip is activated or not. The probability of cross-slip of a screw segment with length $L$ 
in the discrete time step is determined by an activation energy $V_{a c t}\left(|\tau|-\tau_{I I I}\right)$ and the resolved shear stress on the cross-slip plane $\tau$,

$$
P=\beta \frac{L}{L_{0}} \frac{\delta t}{\delta t_{0}} \exp \left[-\frac{V_{a c t}}{k T}\left(|\tau|-\tau_{I I I}\right)\right],
$$

where $\beta$ is a normalization constant, $k$ is the Boltzmann constant, $T$ is set to room temperature, $V_{a c t}$ is the activation volume, and $\tau_{I I I}$ is the stress at which stage-three hardening starts. For $\mathrm{Cu}, V_{a c t}$ is equal to $300 b^{3}$ with $b$ the magnitude of Burgers vector, $\tau_{I I I}=32 \mathrm{MPa}$, and $L_{0}=1 \mu \mathrm{m}$ and $\delta t_{0}=1 \mathrm{~s}$ are reference values for the length of the cross-slipping segment and for the time step [11].

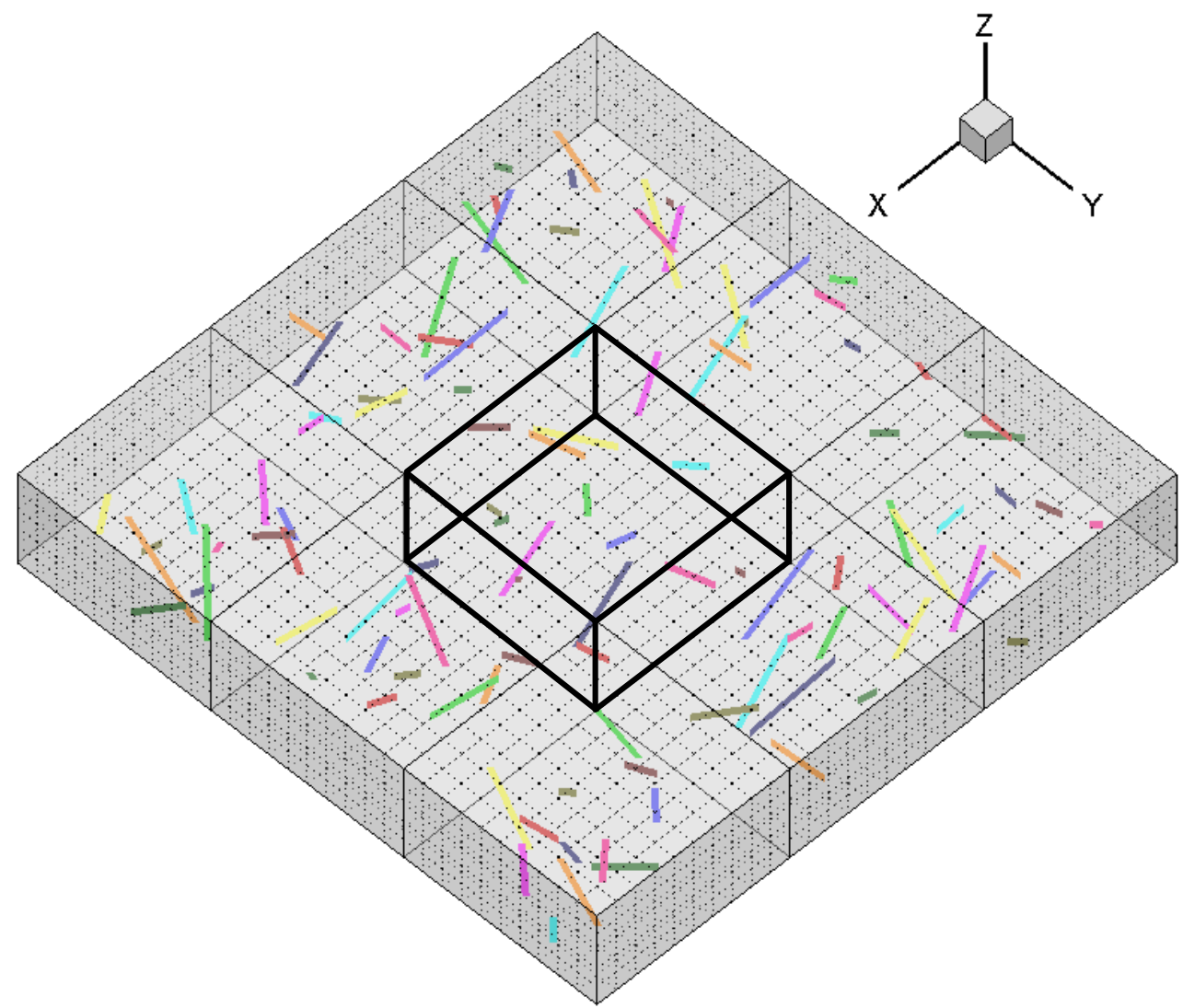

Figure 7.1 Plot of the nine grain aggregate in DD simulations (Dashed lines are BEM mesh and dislocations are in color) 
In this study, a volume element consisting of nine $(=3 \times 3 \times 1)$ columnar grains is set up and represents freestanding polycrystalline thin films as shown in Figure 7.7.1. Each grain has the same size and is set in [001] directions. The cross-section of each grain is square, and the length of each side of the square represents grain sizes (D) while the height of each grain is equal to the thickness of thin films $(\mathrm{H})$. Six sides of the grain aggregate are set as free surfaces from which dislocations can escape. At the beginning of simulations, each grain contains a set of Frank-Read sources with random lengths on twelve $<011>\{111\}$ slip systems. All initial dislocation densities of following simulations are set around $1.0 \times 10^{13} \mathrm{~m}^{-2}$. In our simulations, the grain sizes, $D$, were set to $250,500,1000$ and $1500 \mathrm{~nm}$, and the film thickness, $H$, varies from $250 \mathrm{~nm}$ to $1500 \mathrm{~nm}$ for each grain size. We performed ten calculations with different initial dislocation configurations on each sample and then the results on the same dimension are averaged.

In polycrystals, dislocation may be blocked, reflected, absorbed or transmitted at grain boundaries. The interactions between dislocations and grain boundaries (GB) have been studied by several groups using transmission electron microscopy [12-15]. On the basis of their findings, three conditions have been developed to predict the transmission of dislocations across grain boundaries: (i) the angle between the incoming and outgoing slip planes should be a minimum; (ii) the magnitude of the Burgers vector of the residual dislocation left in the grain boundary should a minimum; (iii) the resolved shear stress on the outgoing slip planes should be a maximum. Recently, de Koning and coworkers [16-17] developed a line tension (LT) model that can capture the essential features of dislocations slipping transmission across grain boundaries and is compatible with the molecular dynamics simulations and experimental results. The LT model considered the transmission as the similar way with operating a FR source on the grain boundary between the incoming and outgoing grains as illustrated in Figure 7.2. The GB transmission strength $\tau_{G B}$ can be rationalized as relatively simple functional relationships between the GB geometry and loading conditions. In our simulations, all grain boundaries are considered as pure tilt 


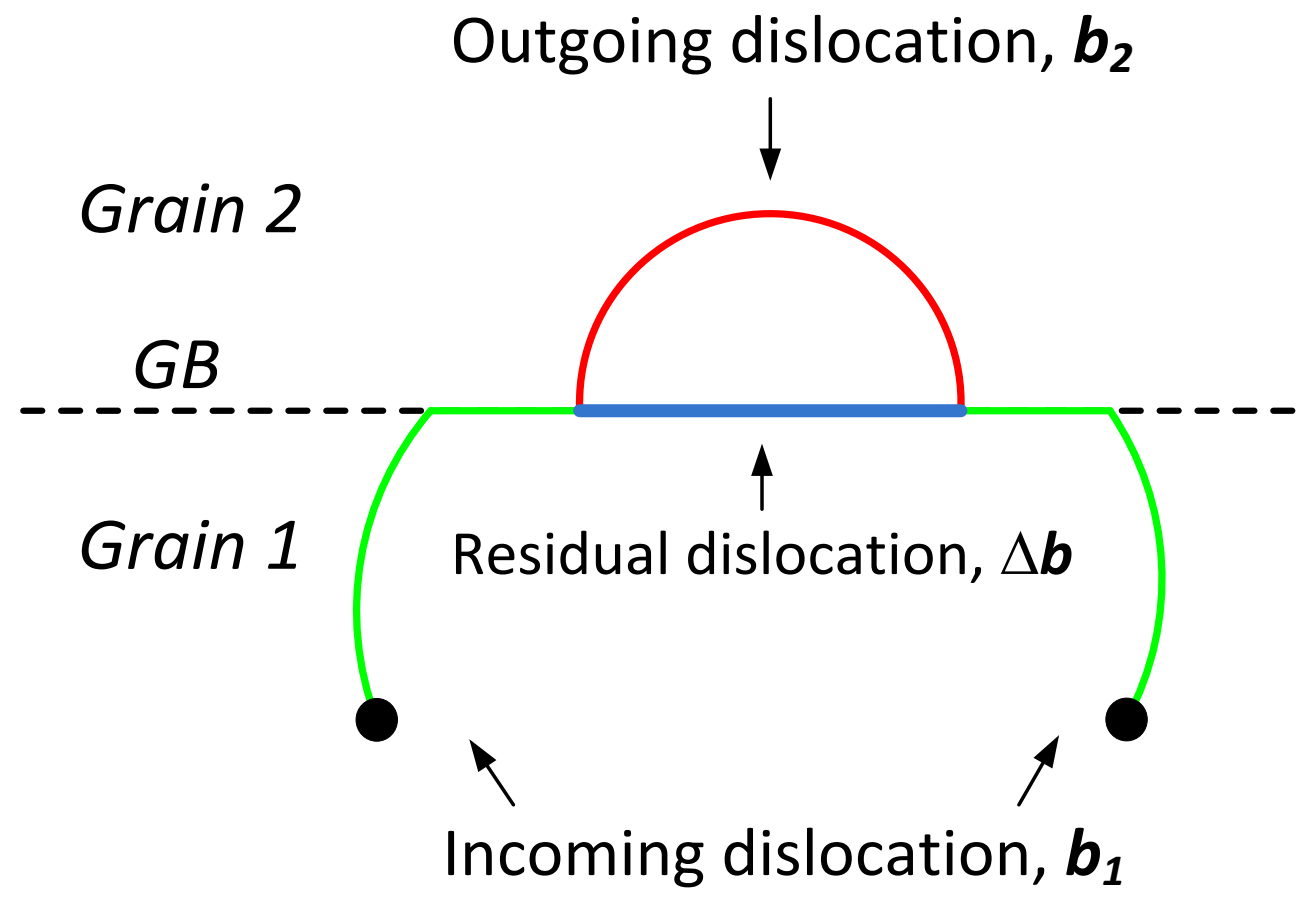

Figure 7.2 Illustration of a dislocation transmitting the tilt grain boundary according to the LT model: the incoming dislocation in the Grainl with Burgers vector, $\boldsymbol{b}_{\boldsymbol{1}}$, gradually bows out under the applied shear stress and then deposits a line segment along the GB; when the resolved shear stress at the GB dislocation exceeds the GB transmission strength, transmission occurs by punching a part of this deposited dislocation line onto Grain2 with Burgers vector, $\boldsymbol{b}_{2}$, and left a residual dislocation with Burgers vector, $\Delta \boldsymbol{b}=\boldsymbol{b}_{2}-\boldsymbol{b}_{1}$, in the GB plane to ensure conservation of the Burgers vector. (Details on the LT model were described in Ref [17]).

boundary, and the Burgers vector of outgoing dislocation segment is equal to that of incoming dislocation segment so penetration does not require the formation of residual dislocation in the GB plane. In this case, the GB transmission strength $\tau_{G B}$ compared with the critical stress to activate the FR source is in the range $\tau_{G B} / \tau_{F R} \approx 2, \ldots, 10$ [17-18]. When the resolved shear stress at the GB dislocation exceeds the GB transmission strength, the GB dislocation will transmit the grain boundary and continue operating in the outgoing grain. In section 3.1, we will compare simulation results with experiment results in Ref [19] to figure out the suitable GB transmission strength in our study. 
In our simulations, tensile loading was applied on the grain aggregate in [100] direction with a constant strain rate equal to $2000 \mathrm{~s}^{-1}$. In order to mimic the plastic deformation in real polycrystalline thin films, we tracked the stress-strain evolution in the center grain of the aggregate marked in Figure 7.1 and averaged the simulation results from a series of simulation results from different initial dislocation configurations to investigate the size-dependent plasticity in polycrystalline thin films.

\subsection{Validation of simulation results}

In order to study the effect of dislocation transmission on the plasticity of thin films and get proper parameters of GB transmission strength in our simulations, we firstly performed simulations on three types of grain boundaries: (a) $\tau_{G B} / \tau_{F R} \rightarrow \infty$, representing impenetrable $\mathrm{GB}$; (b) $\tau_{G B} / \tau_{F R}=n, n$ from 2 to 10 , representing penetrable GB; (c) $\tau_{G B} / \tau_{F R}=0$, representing free GB without resistance on dislocations. In all validation simulations, the grain size is set to $500 \mathrm{~nm}$ and the thickness of thin films is equal to $600 \mathrm{~nm}$, and 15 calculations with different initial dislocation configurations have been completed on each grain boundary condition. A comparison of the computational results for the three types of grain boundaries is shown in Figure 7.3a. To facilitate comparison between the computed and experimental stress-strain curves, the experimental curve from Ref. [19] is also plotted together in Figure 7.3a. It is clear that, when $\tau_{G B} / \tau_{F R}=5$, the computed and experimental stress-strain curves agree quite well and a good fit is obtained to the plateau regime in the stress-strain curves, while the curve for impenetrable GB conditions exhibits nearly linear hardening after initial yielding and the curve for free GB conditions has lower yielding point and flow stress. In Figure $7.3 \mathrm{~b}$, the average dislocation density is plotted against the strain for the three difference cases. We can see the impenetrable GB case has the highest dislocation density and rate of densities increasing, while the dislocation density for free GB case always keeps at the lowest level without any substantial increase. For the penetrable GB case, the 


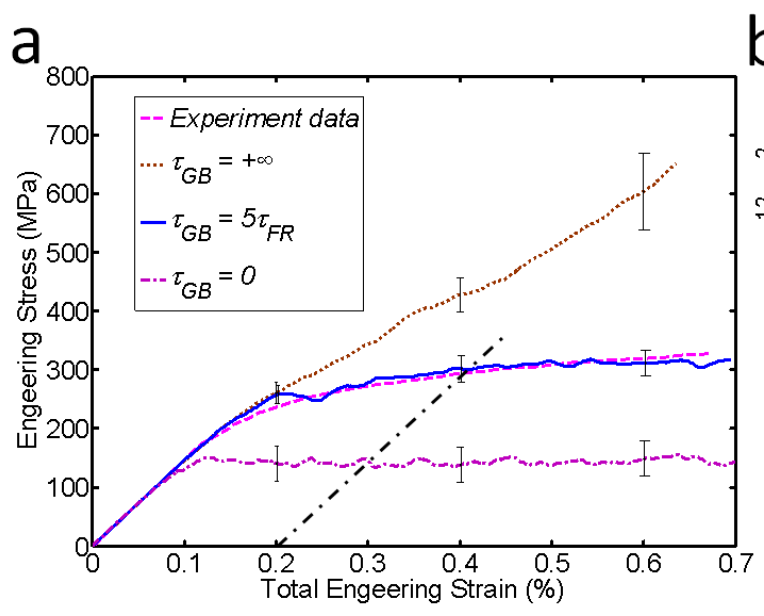

b
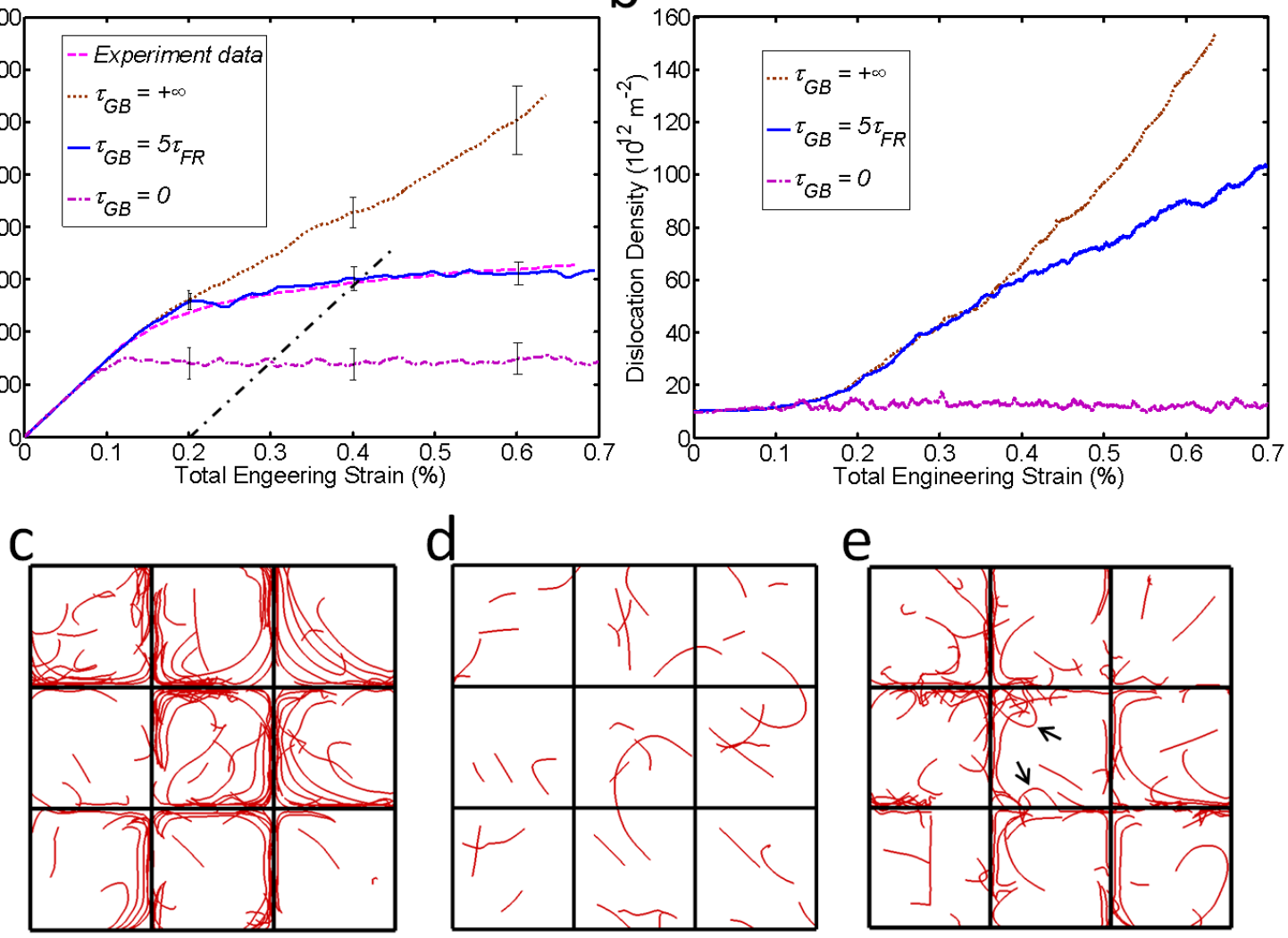

Figure 7.3 Comparison of simulations results with experiment results from Ref. [19]: (a) stress-strain curves of simulation and experimental results on polycrystalline thin films with 500 $\mathrm{nm}$ grain size and $600 \mathrm{~nm}$ thickness; (b) evolution of dislocation densities; (c) dislocation structures in impenetrable GB case, (d) dislocation structures in free GB case; (e) dislocation structures in penetrable GB case with markers on transmitting GB dislocation sources. (Viewing along the [001]-direction).

dislocation density initially has the same increasing rate with impenetrable GB case, after yielding, the increasing rate decreases and the difference of dislocation densities between impenetrable and penetrable cases gradually becomes large with strain increasing. Figure 7.3 c-e shows the typical dislocation structures in polycrystalline films with three different GB conditions after deformed to the same strain level, 0.6\%. For the impenetrable GB case shown in Figure 7.3c, the initial activated dislocations were blocked at the interface when they arrived there. Dislocations activated subsequently are repelled in their progress toward 
the interface by those blocked earlier, and dislocation pile-ups are formed, producing a back stress. Dislocation spacing in the pile-ups is smaller near the interface than it is further away. These pile-ups can effectively reduce the free path of mobile dislocations in the grain and induce hardening with increasing strain. That is why the dislocation-strain curve of impenetrable GB case increasing much faster with increasing strain than the other two cases and the corresponding stress-strain curve exhibits very high strain hardening rate in Figure 7.3a. On the contrary, in free GB case the interface between grains is very clean as shown in Figure $7.3 \mathrm{~d}$, since no resistance exists in the boundary. The corresponding dislocation density just has small fluctuations and almost keeps in the same density level as the initial condition in Figure 7.3b, and the flow stress is generally characterized by the stress required to continuously operate internal dislocation sources to generate the given plastic strain rate. Between these two extreme cases, penetrable GB can trap a certain amount of dislocation sources at the interface, but not permanently. When the resolved shear stress on the GB dislocation exceeds the GB transmission strength, the GB dislocation will transmit the interface and formed a GB source on the adjacent grain as marked in Figure 7.3e. These transmissions of dislocations in grain boundaries can relax the internal stress in grains and reduce the back stress on subsequently activated sources, thus much fewer dislocation pile-ups can form in the penetrable GB case. The comparison between simulation and experimental results in Figure 7.3 indicates that the free and impenetrable GB conditions in DD simulations will under- and overestimate the strength of polycrystalline films, respectively. This can explain why the stress-strain curves in previous 3-D DD simulations on polycrystalline thin films [20-22] always exhibited very high strain hardening rate without plateau regime in the stress-strain curves at larger strains, which idealized grain boundaries as permanent impenetrable. According these validation results, GB transmission strengths, $\tau_{G B}$, are all set to equal to five times $\tau_{F R}$, irrespective of grain sizes and film thicknesses in the following simulations. In addition, the $0.2 \%$ offset yield strength, $\sigma_{y}$, is defined by the intercept of the dash dotted line in Figure 7.3on stress-strain curves. 


\subsection{Grain size dependent strength}
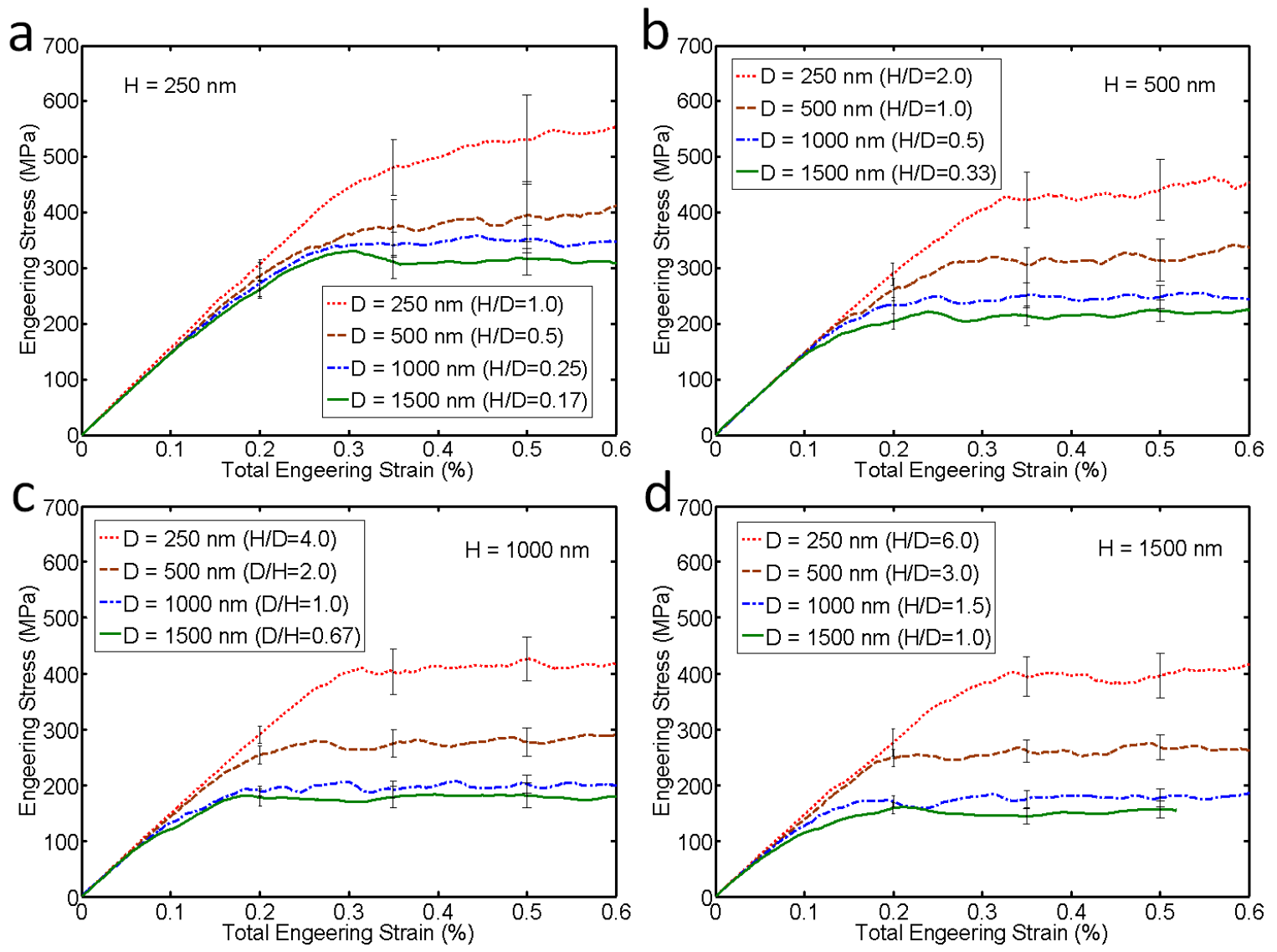

Figure 7.4 Stress-strain plots comparing grain sizes at 250, 500, 1000 and $1500 \mathrm{~nm}$ for film thicknesses of (a) $250 \mathrm{~nm}$; (b) $500 \mathrm{~nm}$; (c) $1000 \mathrm{~nm}$ and (d) $1500 \mathrm{~nm}$.

In order to analysis the effect of grain sizes on the strength of polycrystalline films at a given film thickness, the stress-strain curves for films 250, 500, 1000 and $1500 \mathrm{~nm}$ thick are plotted in Figure 7.4a-d, respectively. Each plot shows the effect of grain size on mechanical response of thin film with grain sizes of 250, 500, 1000 and $1500 \mathrm{~nm}$. Vertical bars on each signature represent data scatter over ten to ten identically sized samples. There is a significant scatter in the magnitude of the flow stress on all grain sizes with decreasing grain sizes in Fig.4. An explanation of this behavior arises from considering that the number of dislocation 

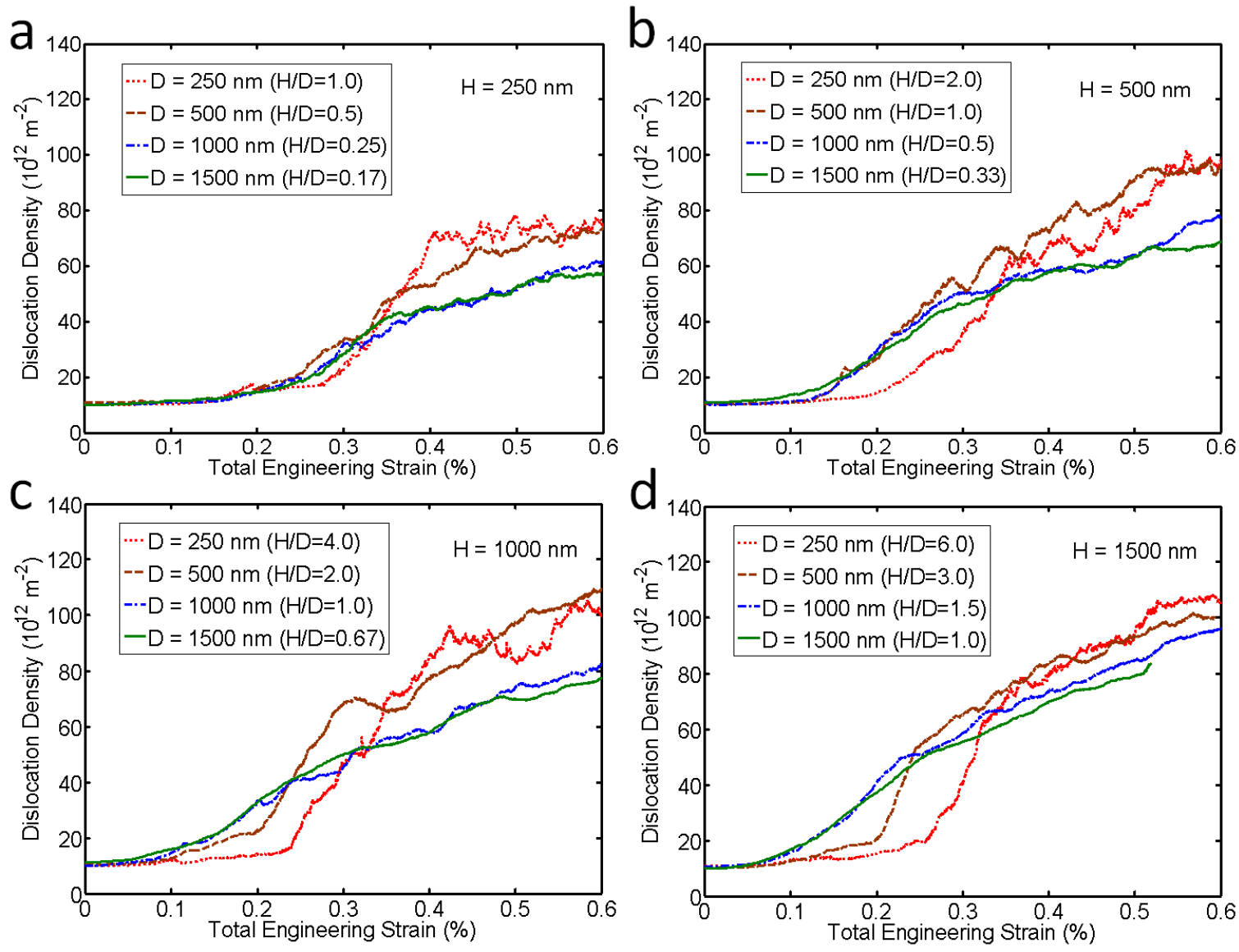

Figure 7.5 Plots of total dislocation density vs. total strain in films with thicknesses of (a) $250 \mathrm{~nm}$; (b) $500 \mathrm{~nm}$; (c) $1000 \mathrm{~nm}$ and (d) $1500 \mathrm{~nm}$.

sources in grains decreases with grain sizes at a given dislocation density. Thus, the statistical effect on the number of dislocation sources in grains will be more obviously reflected on the mechanical response of films with smaller grains [23]. In Figure 7.4, the grain size dependent behavior is remarkably exhibited that, with the grain size decreasing, the flow stress increases irrespective of the film thickness and the strain value, and also the yielding point was delayed to larger strain in smaller grains. The corresponding evolution of total dislocation density and GB dislocation density are plotted in Figure 7.5 and 6, respectively. From Figure 7.5, we can see, the total dislocation increases with increasing strain in all cases and the density-strain curves for large grains are smoother than for small grains. It is 

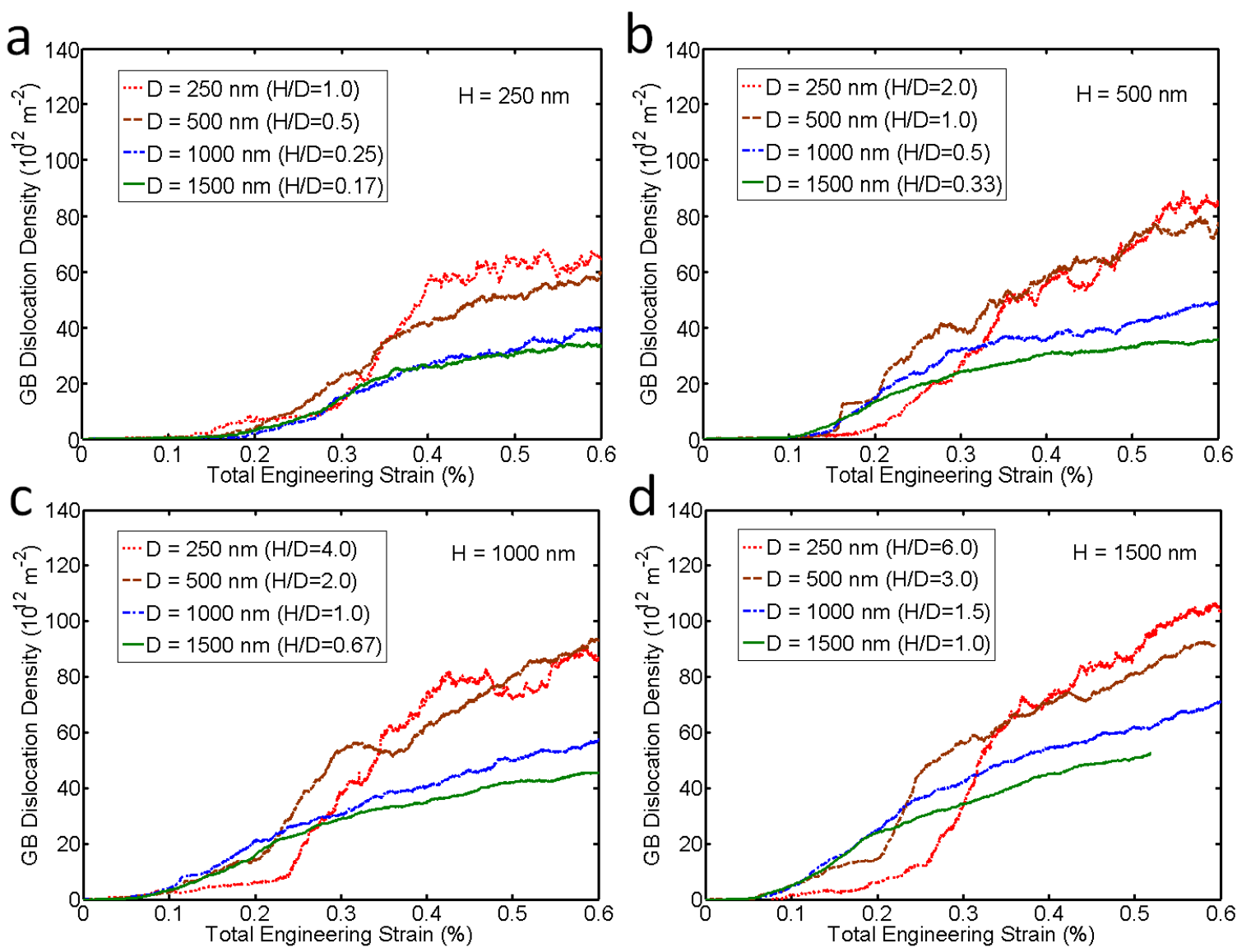

Figure 7.6 Plots of GB dislocation density vs. total strain in films with thicknesses of (a) $250 \mathrm{~nm}$; (b) $500 \mathrm{~nm}$; (c) $1000 \mathrm{~nm}$ and (d) $1500 \mathrm{~nm}$.

interesting that there is one density jump in all samples after yielding indicating large number of dislocation sources have been generated or activated after yielding. The slope of the dislocation density jump is steeper for small grains than for large grains and the jump happened earlier in samples with larger grains since they were yielded earlier. Generally, the total dislocation densities at post-yielding region for $250 \mathrm{~nm}$ and $500 \mathrm{~nm}$ grains are in higher levels, while the other two are in lower levels. Recently, Hommel and Kraft [24] conducted experiments on the deformation behavior of thin copper films and found dislocation density decreases with increasing grain size, which agrees with the trend observed in our simulations. In Figure 7.6, GB dislocation densities in different cases have been plot against the total 

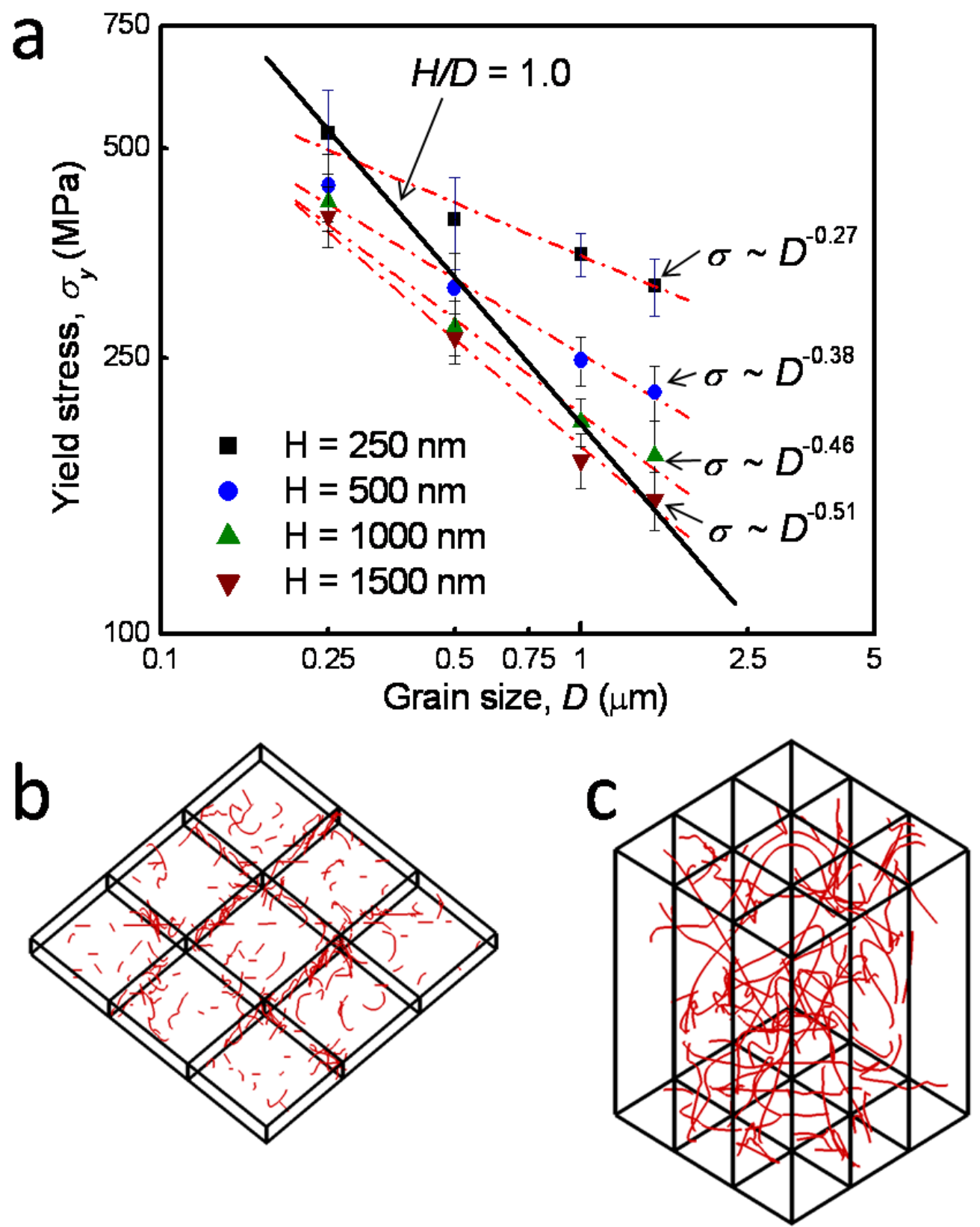

Figure 7.7 (a) Plot of yield stress vs. grain size, D, for the four film thicknesses. Solid line connecting the data points taken from samples with aspect ratio equal to one, above and below which data are taken from samples with low aspect ratio $(<1.0)$ and high aspect ratio $(>1.0)$, respectively; (b) dislocation structures in the film with low aspect ratio, $(\mathrm{D}=1000 \mathrm{~nm}, \mathrm{H}=250$ $\mathrm{nm}$ and $\mathrm{H} / \mathrm{D}=0.25)$ and (c) dislocation structures in the film with high aspect ratio, $(\mathrm{D}=250 \mathrm{~nm}$, $\mathrm{H}=1000 \mathrm{~nm}$ and $\mathrm{H} / \mathrm{D}=4.0$ ). 
strain. The evolution of GB dislocation densities in Figure 7.6 has similar increasing trend with total dislocation denies in Figure 7.5, such as the position and slope of dislocation jumps. At a constant film thickness, the GB dislocation density increases with grain size decreasing and also the portion of GB dislocation densities in the total densities increase. For samples with $250 \mathrm{~nm}$ grain size in all thickness, the fluctuations of GB dislocation density and total dislocation density have almost the same pace and the GB dislocation density almost approaches $90 \%$ of the total densities. That because, at a constant film thickness, reducing grain sizes will increase the GB surface area per unit of grain volume inducing that the free path for dislocations is largely constrained by the grain boundaries, once activated, mobile dislocations will be quickly trapped by the boundary. Hence, the total dislocation densities were most composed of GB dislocation densities in films with smaller grains. Figure 7.6 indicates a general trend that the films with smaller grains held higher GB dislocation densities than larger grain films did at the post-yielding region.

According to the well know Hall-Petch relation based on dislocation pile-up model [25-26], the refinement of grain size can produce stronger polycrystalline materials and the yield strength of the materials should linearly depend on the inverse of the square root of the grain size. To quantitatively assess the size effect observed in Figure 7.4, the $0.2 \%$ yield strength is plotted in Figure 7.7a as a function of grain size, $D$, on a Log-Log scale. This plot clearly shows that the simulation results on films with thickness at 1000 and $1500 \mathrm{~nm}$ can be fitted by the typical form of Hall-Petch relation, $\sigma=\sigma_{0}+k D^{-0.5}$, with scaling exponents approaching 0.5, while the scaling exponents are relative smaller for thinner film with thickness at 250 and $500 \mathrm{~nm}$. In addition, the scaling exponent decreases with a reduction in film thickness from 0.51 for $1500 \mathrm{~nm}$ thickness to 0.27 for $250 \mathrm{~nm}$ thickness. This trend implies that the dependence of yield strength on the grain size gradually becomes weaker with decreasing film thickness. The reason is that most of grains in 250 and $500 \mathrm{~nm}$ films have a pancake-like shape with lower aspect ratio, $\mathrm{H} / \mathrm{D}<1.0$ as shown in Figure $7.7 \mathrm{~b}$, while grains in 1000 and $1500 \mathrm{~nm}$ thicker films have a needle-like shape with higher aspect ratio, 
H/D > 1.0, as shown in Figure 7.7c. In pancake-like grains, most of slip planes intersect the free surfaces from where dislocations can escape freely, and the effective free path of mobile dislocation is largely limited by the film thickness rather than grain boundaries. That weakens the grain size dependent behavior in thin film and dramatically brings down the scaling exponent in thinner films. While in needle-like grains, there is a large number of slip planes end at grain boundaries due to the large ratio of GB areas over volumes that raises the probability of mobile dislocations blocked at grain boundaries. Therefore, needle-like grains strength the grain boundary effect in polycrystalline films and make the scaling exponent in thicker films closer to that for Hall-Petch relation, 0.5.

\subsection{Film thickness dependent strength}

Since previous experimental results showed a strength increase for thinner films over the thicker films [23, 27-30], in this section, the simulation results are sorted by grain sizes to analysis the effect of film thickness on the strength of polycrystalline films. The stress-strain curves for films with grain sizes of 250, 500, 1000 and $1500 \mathrm{~nm}$ have been plotted in Figure $7.8 \mathrm{a}, \mathrm{b}, \mathrm{c}$ and d, respectively. Still quite evident, a film thickness effect on the flow stress is presented irrespective of grain sizes that the flow strength increases as the film thickness decreases. In addition, large scatter in the magnitude of the flow stress appears with decreasing film thickness. The explanation for the large scatter in thinner films is the same as for the significant scatter observed in smaller grains in Figure 7.4. Due to scarcity of available dislocation sources in thinner films at a given dislocation density, the stochastic distribution of dislocation source has stronger influence on the variable of flow stress in thinner films than in thicker films [23]. The total dislocation density and GB dislocation density verse strain are presented in Figure 7.9 and 10, respectively. Generally, the evolution of GB dislocation densities has the same pace with total dislocation densities in all thicknesses. In Figure 7.9 and 10, the density-strain curves for thicker films are smoother 

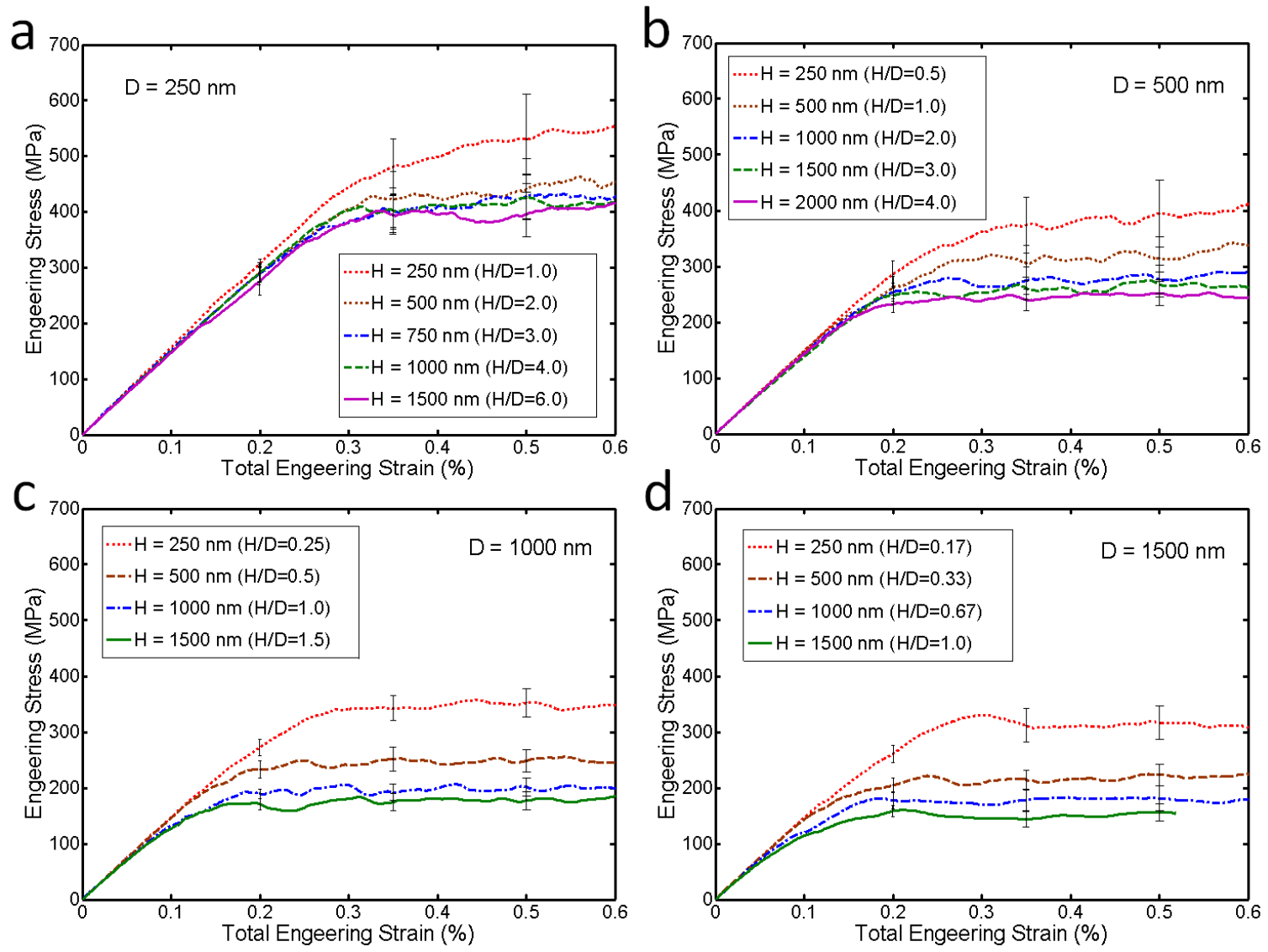

Figure 7.8 Stress-strain plots comparing different film thicknesses for grain sizes of (a) $250 \mathrm{~nm}$; (b) $500 \mathrm{~nm}$; (c) $1000 \mathrm{~nm}$ and (d) $1500 \mathrm{~nm}$.

than for thinner ones, and the dislocation density jump happened earlier in thicker films since they were yielded earlier as shown in Figure 7.8. Compatible with the trend observed in Figure 7.5 and 6 that smaller grains held higher dislocation densities, it is not surprising to find that thicker films stored higher dislocation densities than thinner films did in Figure 7.9 and10. Figure 7.11 shows dislocation structures in films with different thicknesses at the same grain size under the same strain level. It is clear that films with higher aspect ratio stored more dislocations at the grain boundaries. Actually, increasing film thickness at a given grain size and decreasing grain sizes at a given film thickness both will increase the aspect ratio of grains in polycrystalline thin films. In high aspect ratio grains, mobile dislocations have less chance to escape the sample from free surfaces and most of them will 

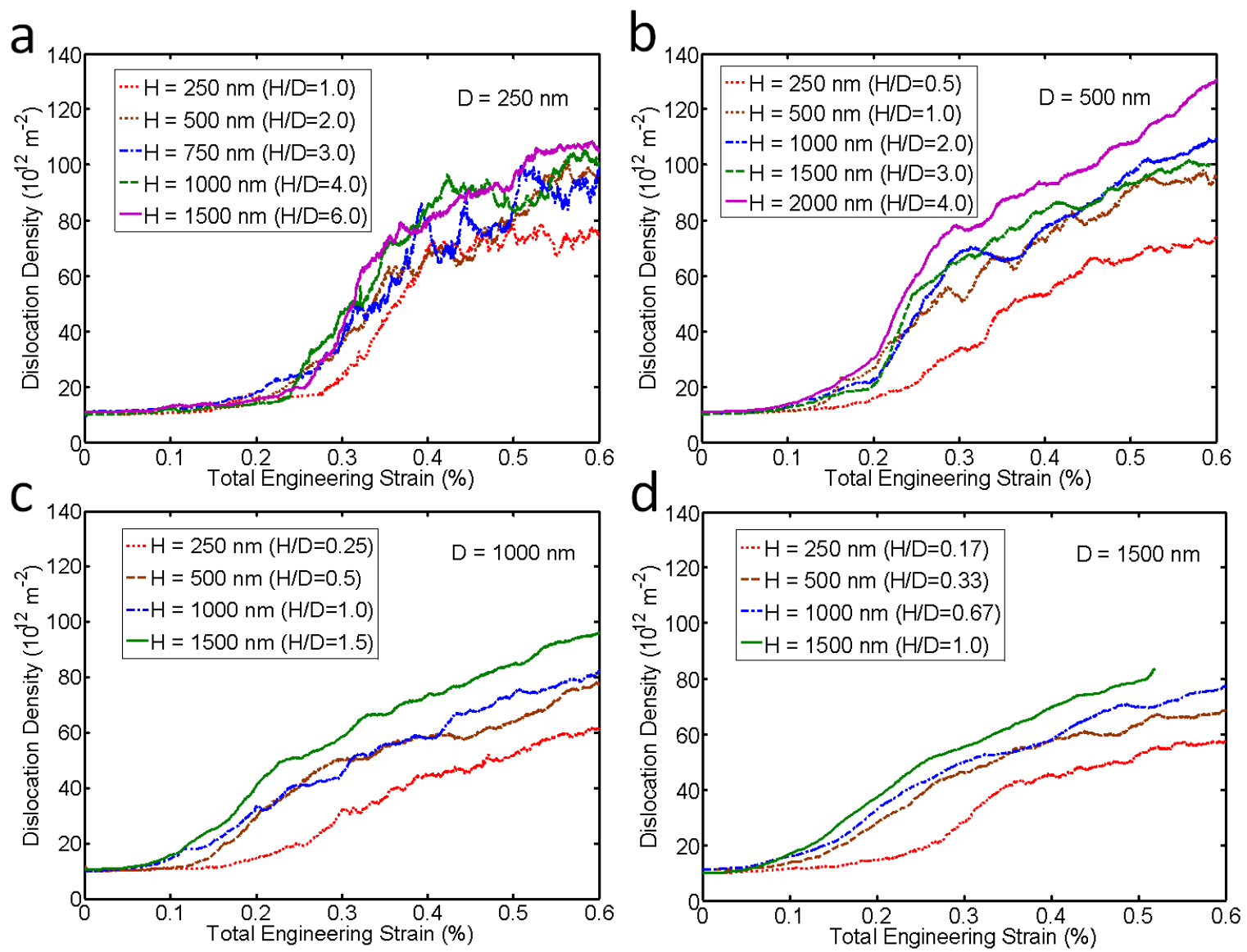

Figure 7.9 Plots of total dislocation density vs. total strain in films with grain sizes of (a) $250 \mathrm{~nm}$; (b) $500 \mathrm{~nm}$; (c) $1000 \mathrm{~nm}$ and (d) $1500 \mathrm{~nm}$.

be blocked by the grain boundaries that can preserve more dislocations in the grain. While it is worth to mention that dislocations near grain boundaries are not necessary to form pile-up under the influence of back-stresses from the source deposited at the grain boundaries, but some of them can relax the repulsive stress by cross-slip to the adjacent plane. Figure 7.12 illustrates one typical example of how the mobile dislocation cross-slip when approaching the grain boundary dislocation. In Figure $7.12 \mathrm{a}$ and $\mathrm{b}$, the mobile dislocation source, $L 2$, with slip system $\frac{1}{2}[101](\overline{1} \overline{1} 1)$ is gradually approaching the previous deposited GB dislocation, $L 1$, with the same slip system on the same plane and experiencing high repulsive stress from $L 1$. In order to relax the high repulsive stress, the screw segment $L 3$ of dislocation $L 2$ escaped the 

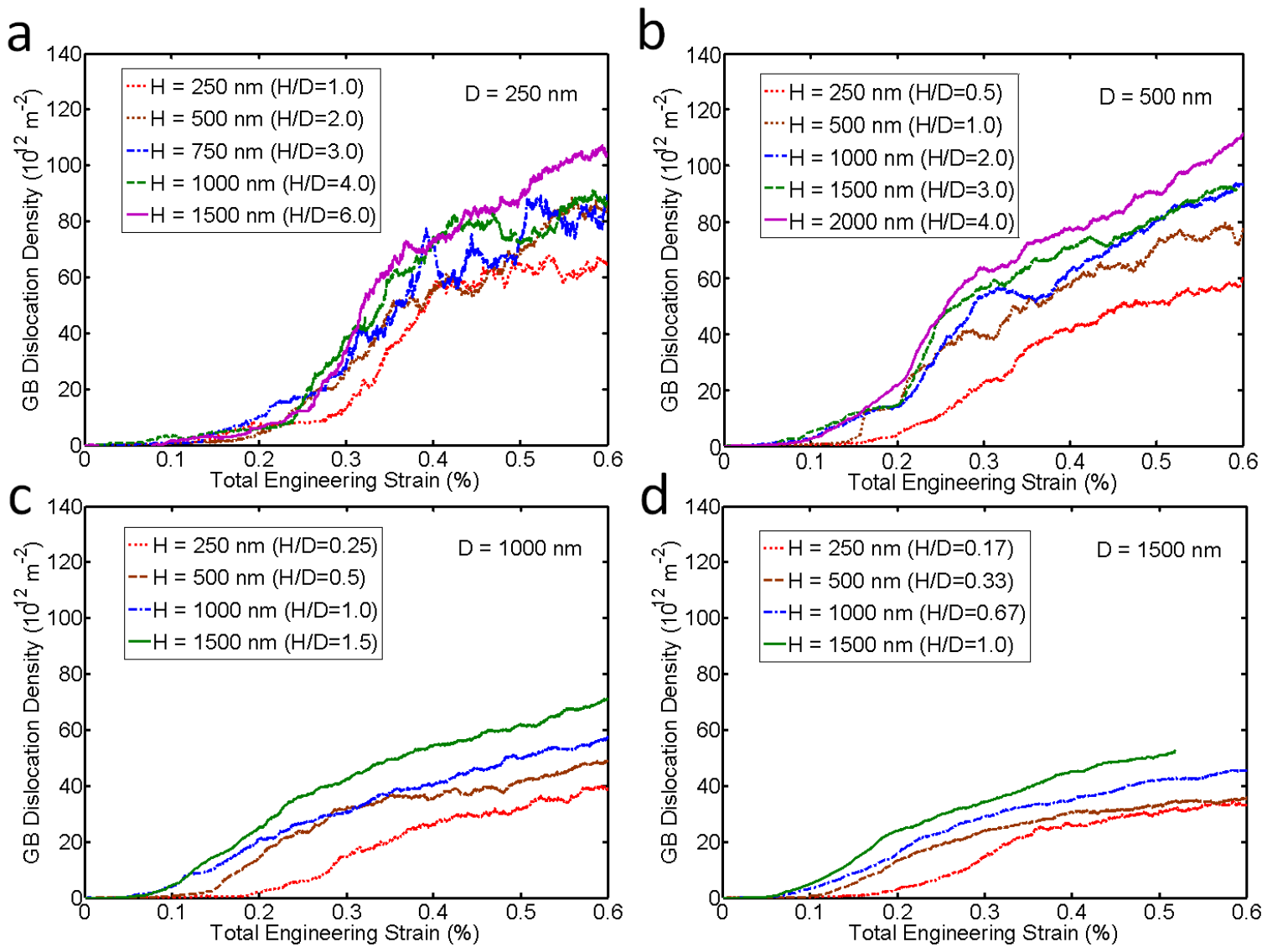

Figure 7.10 Plots of GB dislocation density vs. total strain in films with grain sizes of (a) $250 \mathrm{~nm}$; (b) $500 \mathrm{~nm}$; (c) $1000 \mathrm{~nm}$ and (d) $1500 \mathrm{~nm}$.

original (1̄11) slip plane and cross slipped on the adjacent (111) plane without resistance (Figure 7.12c). Under the applied stress, $L 3$ continued bowing out and finally deposited at the grain boundary, as shown in Figure 7.12d. The whole procedure can be repeated by any mobile dislocations experiencing a high repulsive stress from GB dislocations, generating a large number of dislocations depositing on the grain boundaries, if the area of grain boundary is large enough. This can explain why the GB dislocation density is higher in high aspect ratio films, since they have large grain boundary areas per volume in the film with the same grain size. Recently, Chauhan and Bastawrosa [31] probed dislocation storage in freestanding $\mathrm{Cu}$ films using residual electrical resistivity and found a reduction in film thickness would 

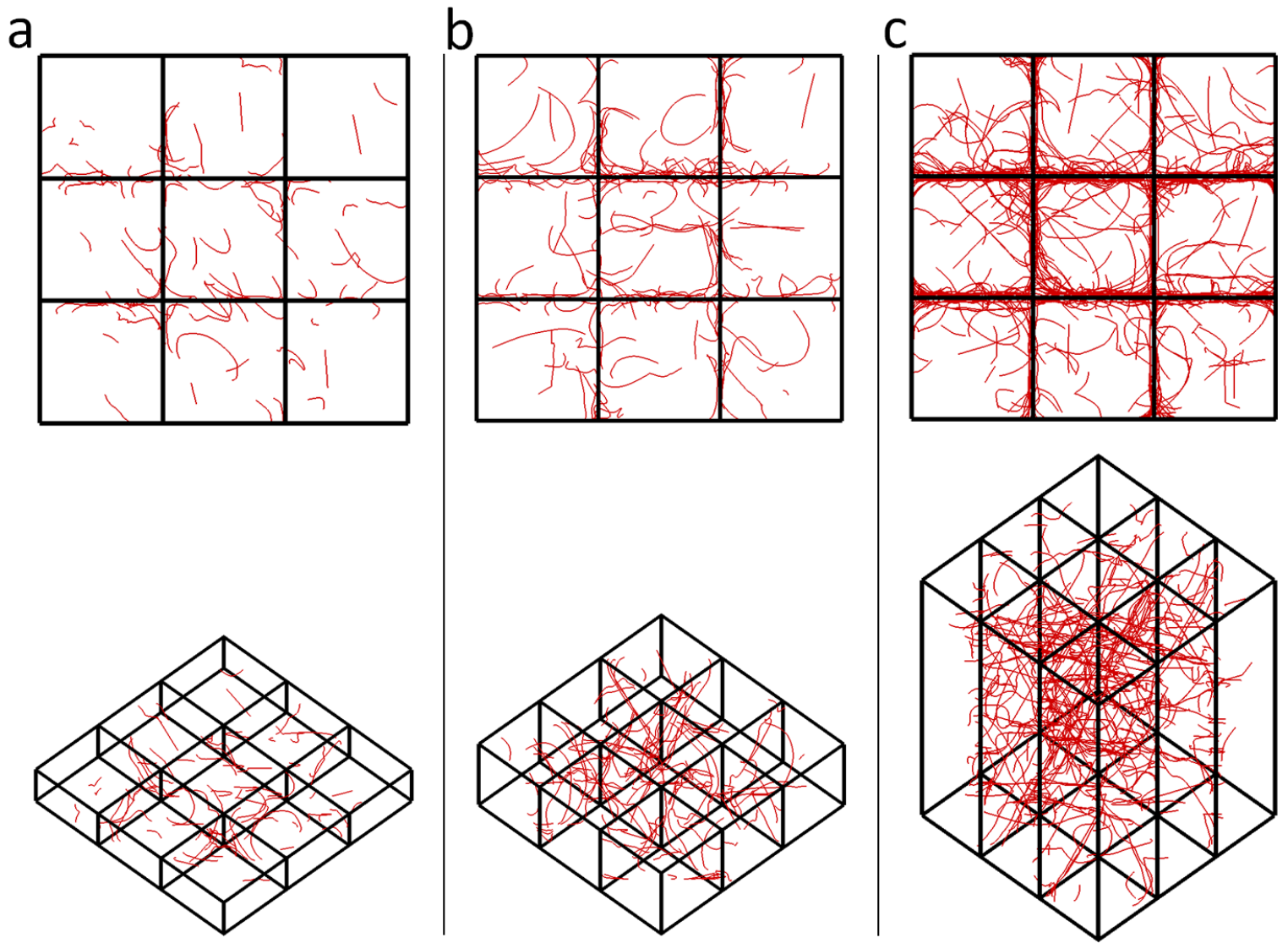

Figure 7.11 Dislocation structures in films with grain size equal $500 \mathrm{~nm}$ under $0.5 \%$ strain in different film thicknesses: (a) thicknesses equal $250 \mathrm{~nm}(\mathrm{H} / \mathrm{D}=0.5)$, upper in [001] view, lower in [ $\left.\begin{array}{lll}1 & 1 & 1\end{array}\right]$ view; (b) thicknesses equal $500 \mathrm{~nm}(\mathrm{H} / \mathrm{D}=1.0)$, upper in [001] view, lower in [1 111 1] view; (c) thicknesses equal $2000 \mathrm{~nm}(\mathrm{H} / \mathrm{D}=4.0)$, upper in [001] view, lower in [ [ 1111$]$ view.

limit GB dislocation sources and decrease dislocation densities in polycrystalline films, which is in agreement with our results. According to the strain-gradient theory [32-34], the accommodated geometrically necessary dislocations can cause lattice curvature and non-homogeneous deformations in crystal materials that induces "smaller is stronger". However, the trend observed in our simulations, thinner films are harder shown in Figure 7.9 and 10 , is not consistent with the strain-gradient theory, since they stored less dislocations density in the grain.

The thickness effect on the film strength shown in Figure 7.8 is further illustrated in Figure 7.13, where the yield stress is plotted as a function of thickness inverse and compared 

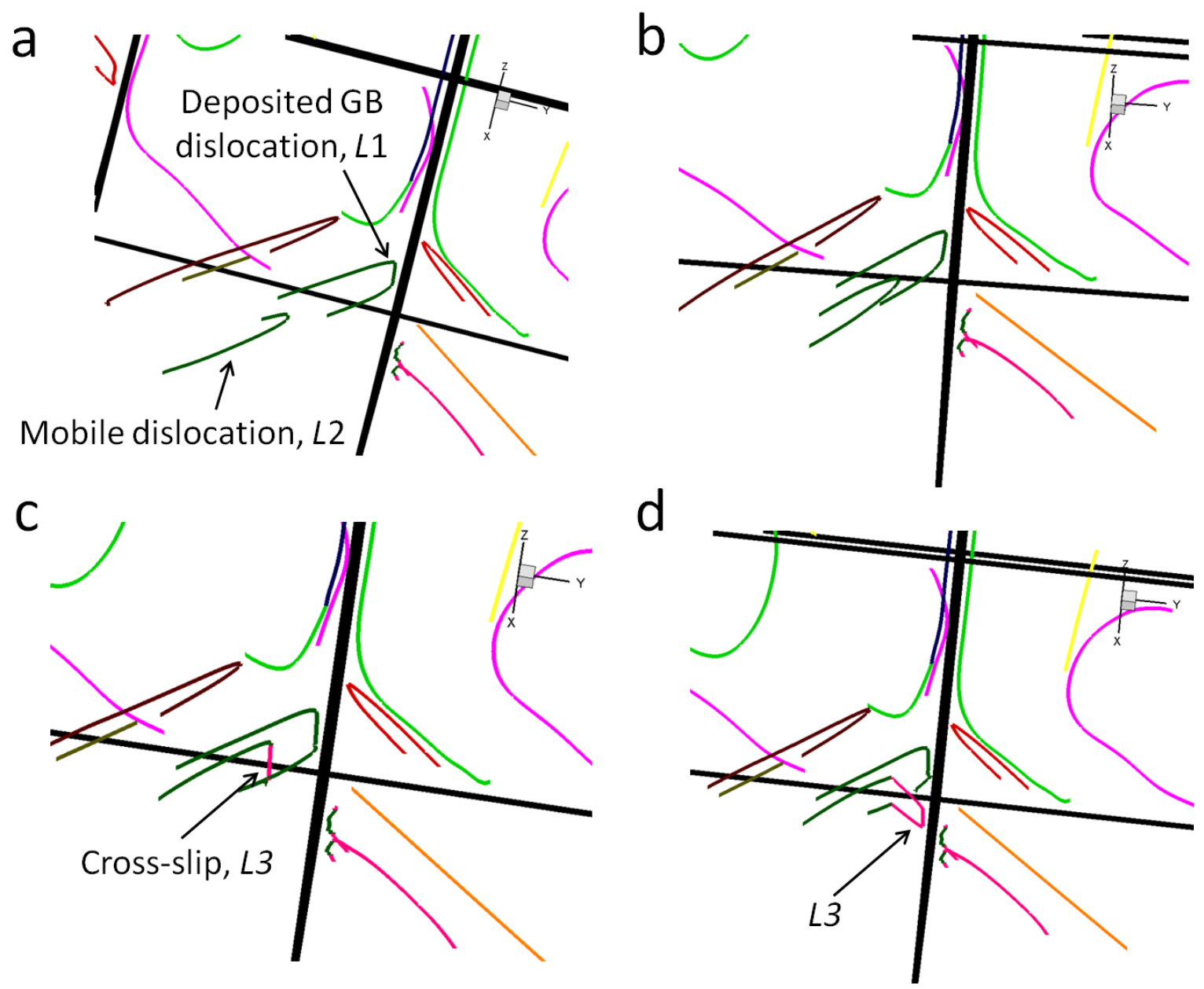

Figure 7.12 Plots of mobile dislocation cross-slip when approaching the grain boundary dislocation: source $L 1$ and $L 2$ with $1 / 2[101](\overline{1} \overline{1} 1)$, source $L 3$ with $1 / 2[101](\overline{1} 11)$, and black lines indicating the grain boundaries, see details in text.

with experiment results on freestanding polycrystalline $\mathrm{Cu}$ films $[19,35]$. For the samples with grains size at 1000 and $1500 \mathrm{~nm}$, we can observe a size effect in which the yield stress scales proportionally to $1 / \mathrm{H}$, while for 250 and $500 \mathrm{~nm}$ films, the thickness dependence is relative weaker. That because most of grains in 250 and $500 \mathrm{~nm}$ films have needle-like grains $(\mathrm{H} / \mathrm{D}>1.0)$ as shown in Figure 7.11c, in which most slip planes in the center grain has been intersected by grain boundaries, and only few of them can touch the top and bottom free surfaces. Since the free path of mobile dislocations will be mainly limited by the smaller 


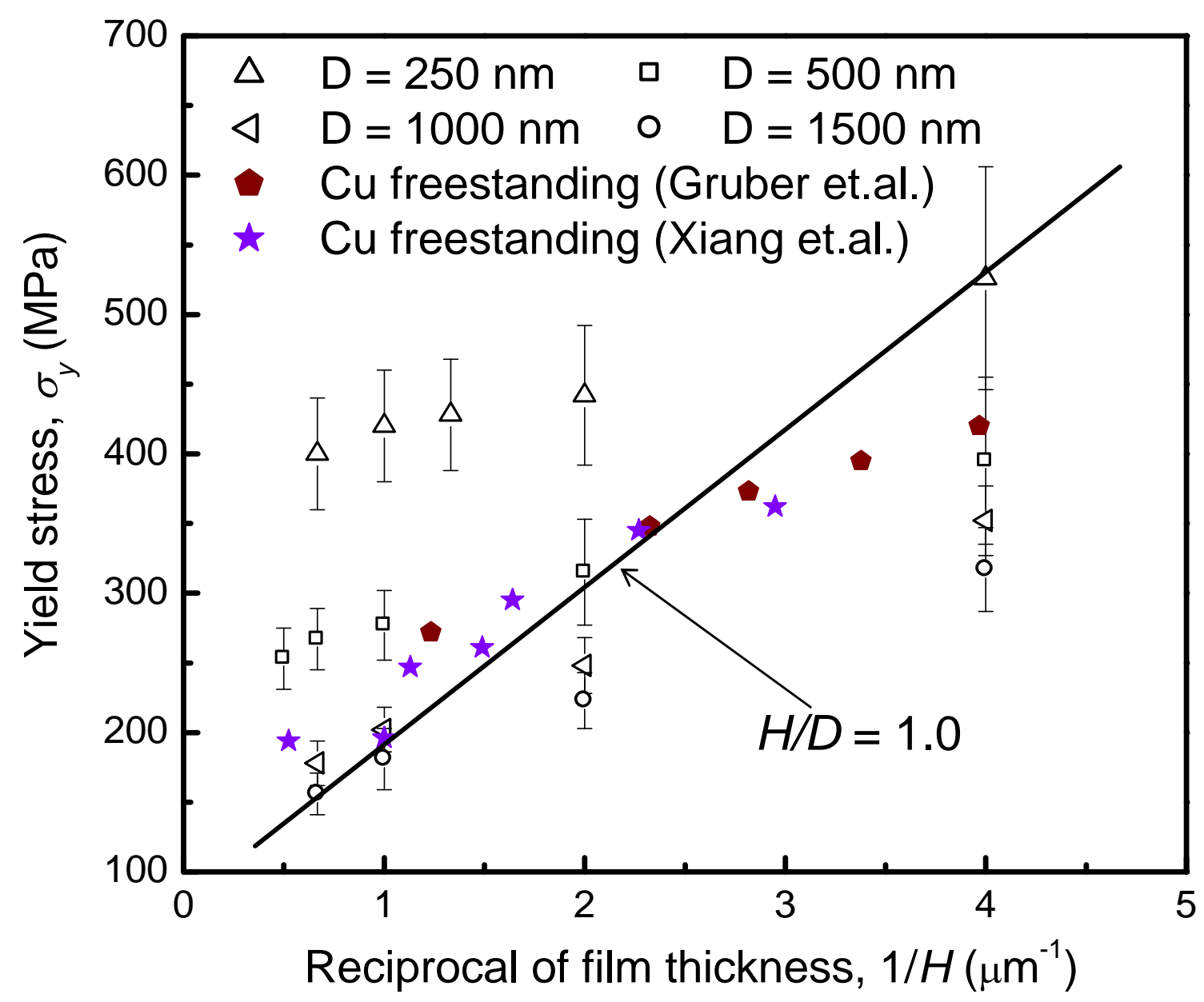

Figure 7.13 Comparison of yield stresses from simulation and experiment results. Solid line connecting the data points taken from samples with aspect ratio equal to one, above and below which data are taken from samples with high aspect ratio $(>1.0)$ and low aspect ratio $(<1.0)$, respectively.

dimension of the sample, the grain size in this case, films thicknesses exhibit a minor effect on the strength of samples. Thus, at a given grain size, the difference of yield stresses is not obvious in films with aspect ratio larger than one, which have been reflected by the larger overlapping error bars in these cases on Figure 7.8a and b. In addition, our simulation results can match experiment results well on the distribution of yield strength in Figure 7.13. Since Xiang's results were taken from samples with aspect ratio slightly larger than one, most of their data points lay above the line, $\mathrm{H} / \mathrm{D}=1.0$. While most of Gruber's results lay below the 
line, as their samples have lower aspect ratios.

\subsection{Spiral source model}

Plastic deformation in thin film can generally be divided into two main categories: (i) nucleation dominated mechanism, i.e. nucleation and absorption of partial dislocations at grain boundaries or interfaces [36-38], which is similar to the mechanism found in nanocrystalline metals, where grain boundaries are highly effective dislocation sinks and sources and perfect dislocation sources cease to operated [39-40]. Actually, the thickness dependence of the nucleation stress of partial dislocations is inherently lower than for perfect dislocations since it strongly depends on the stacking fault energy which is independent of film thickness. That may explain why some experiments found size-independent strength in metallic films with thickness approaching $100 \mathrm{~nm}$ [41-43]. (ii) Multiplication dominated mechanism, i.e. dislocation generating new sources or segments in films to relax the internal stresses to achieve the imposed plastic deformation. Normally, the activating stress required to produce new dislocation sources or segments is limited by confined geometries due to the presence of the substrate or a passivation layer [28] and grain boundaries [44], and thus strongly size-dependent plasticity can be found in the films at micron and submicron regime $[28,45]$. The plastic deformation in this study belongs to the second category, multiplication dominated mechanism, since the film thickness and grain sizes are both in the range of micron and submicron.

In the past decade, the misfit dislocation model [28] has been widely used to explain the plastic deformation in passivated films, in which work done by the applied stress must be enough to bow a dislocation and leave two dislocations at the film-substrate and film-oxide interfaces. As misfit dislocation is not present in the top or bottom of freestanding films, there must be other strengthening mechanism in these films to explain the size effects than the existing one arising from misfit dislocations. von Blanckenhagen et al. [46-47] simulated 

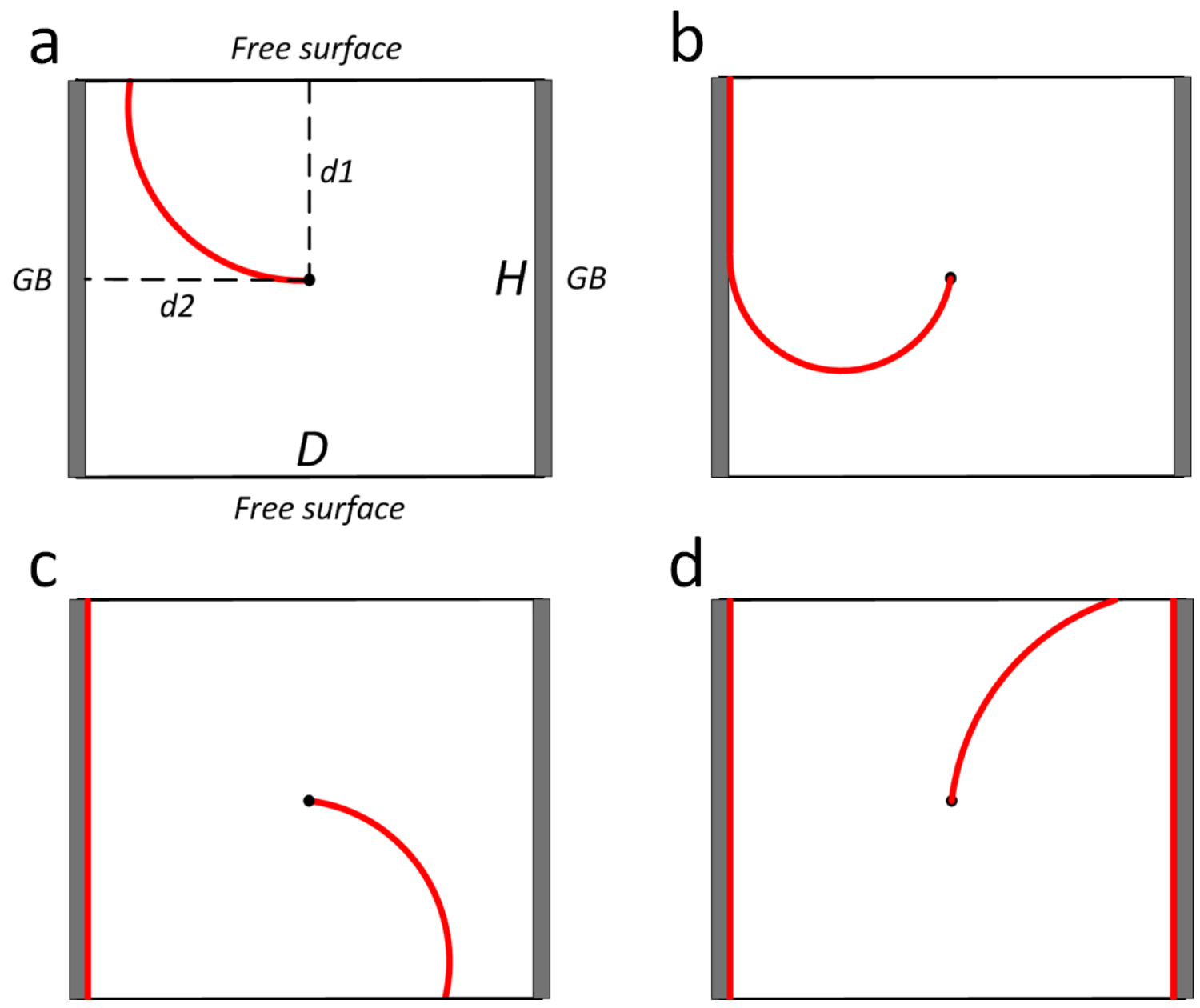

Figure 7.14 Schematic depiction of the operation of spiral source in freestanding thin films. Red lines are dislocations; $\mathrm{d} 1$ and $\mathrm{d} 2$ indicate the shortest distances of internal pinning point to the free surface and grain boundary; the spiral source operates in counterclockwise direction.

thin film plasticity by putting a single Frank-Read source in the center of a columnar grain and concluded that the size of the most effective dislocation source for unpassivated thin films is $1 / 3$ of the smaller dimension among film thickness or grain size. However, the two arms of randomly set Frank-Read sources in our simulation are always blocked by the grain boundary or truncated by free surfaces forming single-ended spiral sources and seldom operate at the same time. Instead, only the one arm with longer distance to the edge of the grain can continue operating and transform back to its initial position to allow further 
activation as shown in Figure 7.14. Recent in-situ TEM observations confirmed our simulation results that the operation of single-ended spiral source was recorded during straining and the emission of new dislocations relaxed the applied strain and stress [43, 48]. As shown in Figure 7.14, when the moving arm approaches the interface, the spiral source can operate like double-ended Frank-Read sources and will deposit dislocation segments on the grain boundary. When the moving arm arrived at free surfaces where they can escape free, the surface node can just slip along the intersection line between its slip plane and the free surface. The stress required to operate a single-ended spiral source have the same form as that for double-ended Frank-Read source [49]. Since the dislocation sources in thin films are rare and just have to operate several times to achieve the imposed plastic deformation, the critical resolved shear stress (CRSS) for initiate yielding in the film can be rationalized by considering the stress required to move the longest spiral sources formed in the sample as following:

$$
\tau=\tau_{o}+k \mu \frac{\ln (\bar{L} / b)}{(\bar{L} / b)}+\alpha \mu b \sqrt{\rho}
$$

where $\tau_{o}$ is the lattice friction stress, $\bar{L}$ is the effective source length, $\mu$ is shear modulus, $b$ is magnitude of Burgers vector, and $k$ is source-hardening constant, equal to 0.12 for single-ended sources and 0.18 for double-ended sources [49], $\alpha$ is the hardening coefficient ( $\sim 0.35$ for FCC metals) [50], $\rho$ is the dislocations density. In this study, we take $\tau_{o}=10 \mathrm{MPa}$, $b=0.25 \mathrm{~nm}$ for $\mathrm{Cu}, \rho=1.0 \times 10^{13} \mathrm{~m}^{-2}$ and the average $k=0.15$. The second and third terms in eq. (7.2) represent the stress required to activate the effective spiral source in the sample and back stress from the dislocation forest, respectively.

Following an idea from ref. [51], the length of effective spiral source can be evaluated from a statistical model illustrated in Figure 7.15. Since most films in experiment showed equiaxed grains, we assume the shape of the gain is square, $H=D$, and the spiral sources 


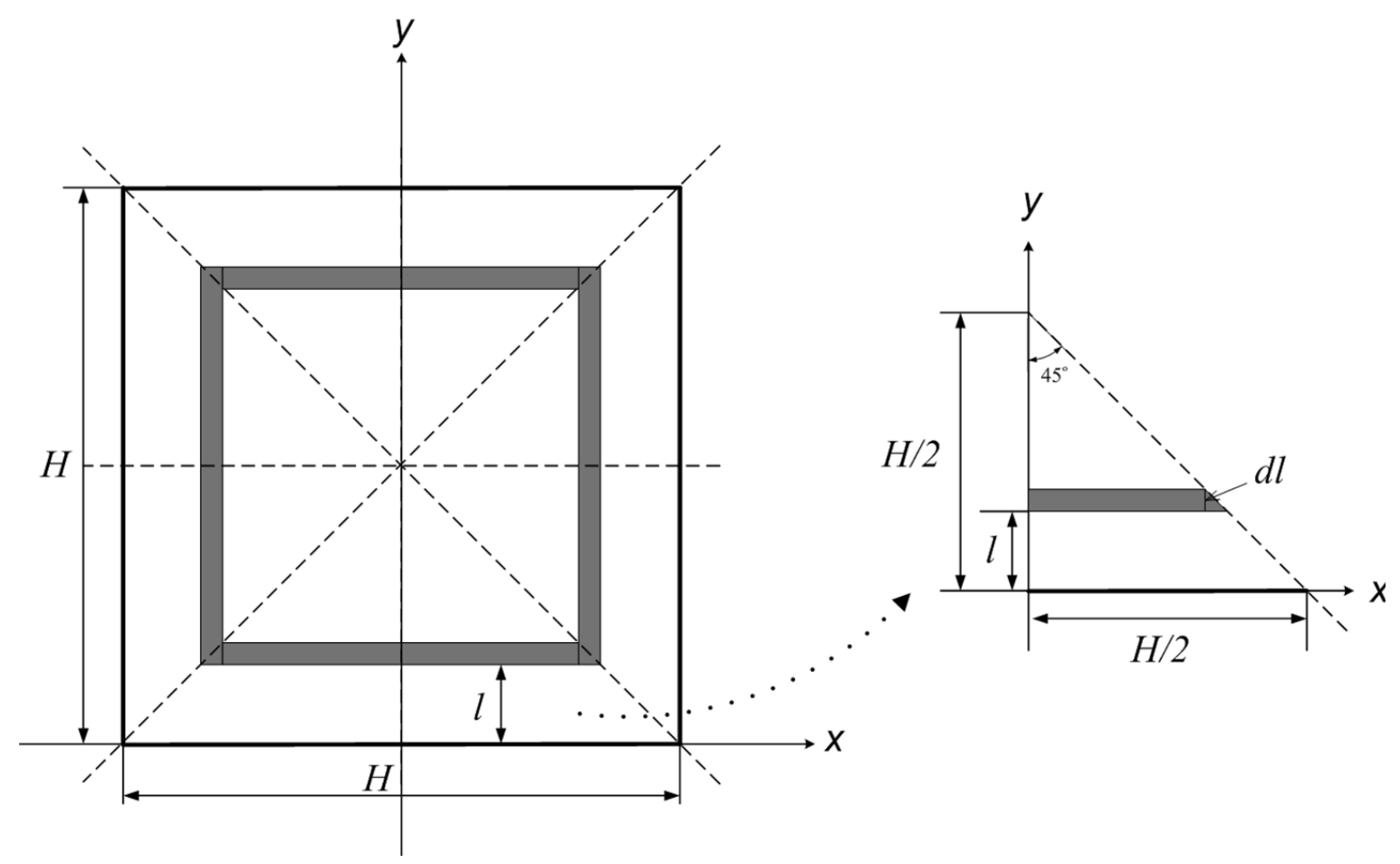

Figure 7.15 Schematic sketch of the statistical model for evaluating the effective length of spiral source in an equiaxed grain. Dashed lines indicate the axis of symmetry in the square.

with internal pins located in the shaded area have the same distance to the edge of square grain. As the square grain is quartic symmetry, we can take out an isosceles right triangle from the square to analysis the problem as shown in Figure 7.15. For a random distribution of pins in the triangle, the probability, $P(l) d l$, of finding a pin within the shaded area in the isosceles right triangle width, $d l$, at a distance $l$ from the edge (bottom) is given by

$$
\boldsymbol{P}(\boldsymbol{l}) d l=\frac{4(H-2 l) d l}{H^{2}}
$$

For the case of $n$ pins located randomly, the probability for the maximum distance from the edge to be $L_{\max }$, is given by 


$$
\boldsymbol{P}\left(\boldsymbol{L}_{\max }\right) d \boldsymbol{L}_{\max }=n \frac{4\left(H-2 \boldsymbol{L}_{\max }\right) d \boldsymbol{L}_{\max }}{H^{2}} \times\left(1-\frac{\left(H-2 \boldsymbol{L}_{\text {max }}\right)^{2}}{H^{2}}\right)^{n-1} .
$$

The above equation gives the probability that a given sample with $n$ pins has $L_{\max }$ as the effective source length. The first moment of this distribution will give the mean effective source length as

$$
\begin{aligned}
\overline{\boldsymbol{L}}_{\text {max }} & =\int_{0}^{\frac{H}{2}} \boldsymbol{L}_{\max } \boldsymbol{P}\left(\boldsymbol{L}_{\max }\right) d \boldsymbol{L}_{\max } \\
& =\int_{0}^{\frac{H}{2}} n \boldsymbol{L}_{\max } \frac{4\left(H-2 \boldsymbol{L}_{\max }\right) d \boldsymbol{L}_{\max }}{H^{2}} \times\left(1-\frac{\left(H-2 \boldsymbol{L}_{\max }\right)^{2}}{H^{2}}\right)^{n-1} d \boldsymbol{L}_{\max } .
\end{aligned}
$$

Thus the second moment of this distribution gives the standard deviation of the effective source length by

$$
\sigma_{\boldsymbol{L}_{\max }}=\left[\int_{0}^{\frac{H}{2}} \boldsymbol{L}_{\max }^{2} \boldsymbol{P}\left(\boldsymbol{L}_{\max }\right) d \boldsymbol{L}_{\max }-\overline{\boldsymbol{L}}_{\max }^{2}\right]^{\frac{1}{2}} .
$$

The number of pins, $n$, is related to the sample dimensions and initial dislocation density in the sample, as given by

$$
n=\operatorname{Integer}\left[\rho_{\text {initial }} \frac{V}{L_{\text {ave }}}\right] \times 2,
$$

where $\rho_{\text {initial }}$ is the initial dislocation density, $V$ is the sample volume, $L_{\text {ave }}$ is the average length of dislocation segments in the sample and the factor two indicates each Frank-Read source has two pins in the sample. In this study, the average length of dislocation segments is taken to be $H / 2$. The upper bound of this spiral source model should be stress required to nucleate dislocations from the free surface or grain boundaries. Since we do not know the 


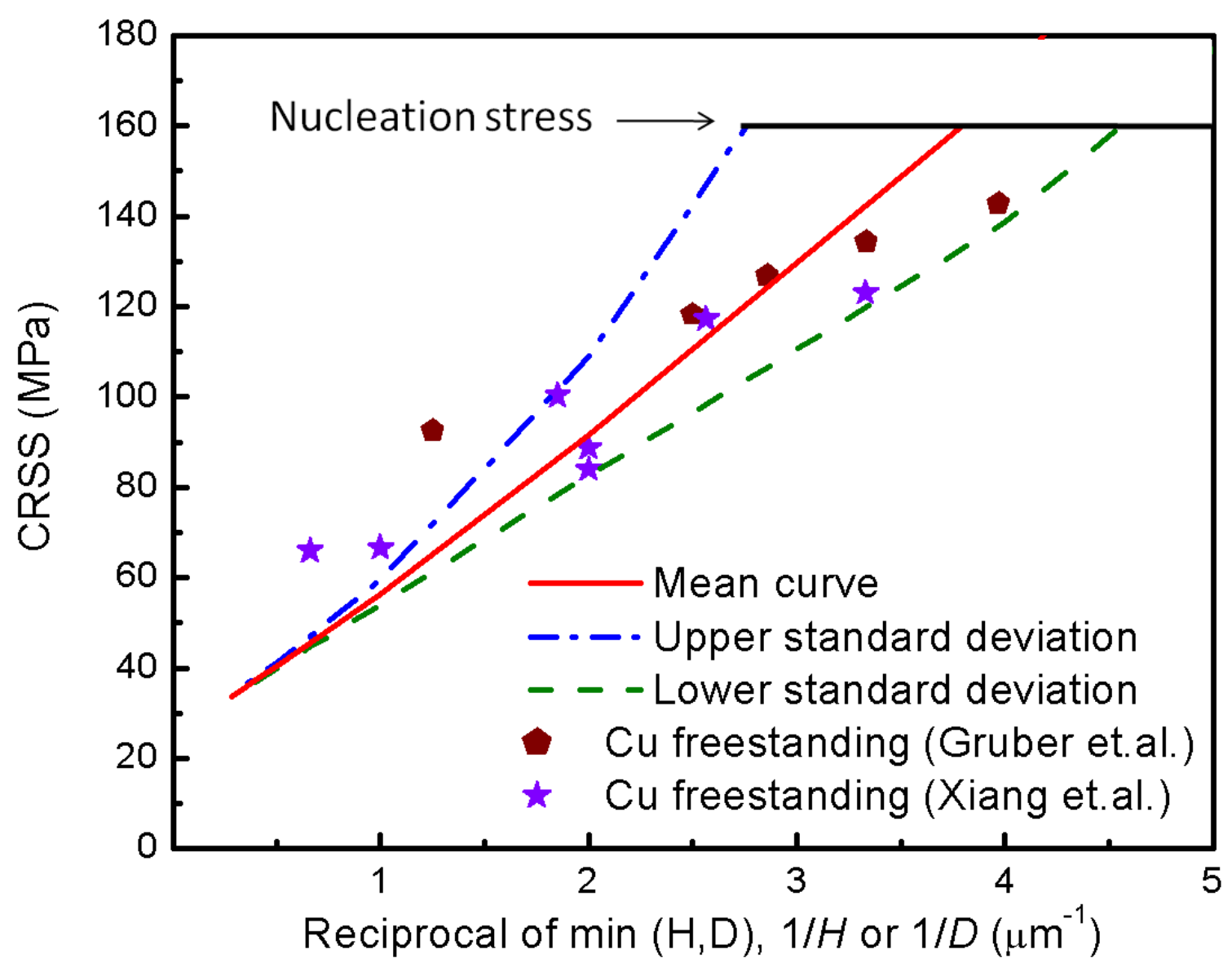

Figure 7.16 Comparison of the results predicted by spiral source model stress with experimental data. The stress is shown versus the reciprocal value of the smaller dimension among film thickness or grain size.

actual stress, we estimated the nucleation stress based on $\gamma / b$, where the stacking fault energy, $\gamma=0.04 \mathrm{~J} / \mathrm{m}^{2}$ for $\mathrm{Cu}[52]$. Thus, the required nucleation is approximately equal to $160 \mathrm{MPa}$.

In Figure 7.16, the results predicted by spiral source model are compared with experimental data for freestanding polycrystalline $\mathrm{Cu}$ films. It can be seen that this spiral source model can reasonably predict the increase of strength with film thickness decreasing and the large scatter in the magnitude of strength in thinner films. For the thicker films, the predicted results are relative lower than that observed in experiments. That because this model just predicts the initial stress for plasticity and neglects hardening from the reactions between mobile sources and forest dislocations. Since the dislocation structures and reactions 
are more complicated in thicker films than those in thinner ones, the effective dislocation sources may be shortened in thicker films resulting in higher strength. In addition, the dislocation density in experiment may vary from one to one. That can also cause the experiment results diverge from the predicted value. It is worth to point out that this model can not only be developed to predict the yield stress for polycrystalline thin films, but also other confined small volumes, such as single crystal thin films and micropillars [51].

\subsection{Conclusions}

In this study, a 3D DD simulation was set up to investigate the plasticity of freestanding polycrystalline thin films. Both cross-slip and grain boundary relaxation mechanisms have been considered in our simulations, which are important in the plastic deformation of polycrystalline films and neglected by previous studies. The simulations were analyzed to identify the evolution of dislocation sources and densities in the presence of free surfaces and grain boundaries at the micron and submicron regime. According to our simulation results, a spiral source model has been established to predict the size-dependent strength in thin films. The findings can be summarized as follows:

The stress-strain curves of freestanding polycrystalline films can be predicted by 3D DD simulations with a probable dislocation transmission rule. The computed and experimental stress-strain curves agree quite well and a good fit is obtained to the plateau regime with penetrable GB condition, while the curve for impenetrable GB conditions exhibits nearly linear hardening after initial yielding and the curve for free GB conditions has lower yielding point and flow stress.

At a constant film thickness, the total dislocation density deceases with increasing grain sizes due to the increment of surface area to volume ratio. In films with pancake-like grains, the dependence of yield stress on the grain size gradually becomes weaker with decreasing film thickness. In contrast, the needle-like grains strength the grain boundary effect in 
polycrystalline films and grain size dependent behaviors in films with high aspect ratio can be described by Hall-Petch relation.

With the same grain size, high aspect ratio films can hold higher total and GB dislocation density, since they have large grain boundary areas per volume in the film. The yield strength of films can scale proportionally to the reciprocal of thickness. With high aspect ratios increases, the dependence of the film strength on thickness becomes weaker due to the effect of free surfaces has been diminished.

According to the dislocation structures observed in our simulations, a spiral source model has been set up to predict the yield stress of thin films. Comparison with data on freestanding thin films shows that spiral source model can reasonably explain the increase of strength with film thickness decreasing and the large scatter in the magnitude of strength in thinner films.

Further investigations are need for studying the plasticity of polycrystalline films with few grains across the thickness and also implement more realistic models on dislocation and grain boundary reactions in 3D DD simulations, both of which will slow down computation significantly. 


\section{References}

[1] Ghoniem NM, Sun LZ. Fast-sum method for the elastic field off three-dimensional dislocation ensembles. Physical Review B 1999;60:128.

[2] Ghoniem NM, Tong SH, Sun LZ. Parametric dislocation dynamics: A thermodynamics-based approach to investigations of mesoscopic plastic deformation. Physical Review B 2000;61:913.

[3] Wang ZQ, Ghoniem N, Swaminarayan S, Lesar R. A parallel algorithm for 3D dislocation dynamics. Journal of Computational Physics 2006;219:608.

[4] Wang ZQ, Beyerlein IJ, Lesar R. Plastic anisotropy in fcc single crystals in high rate deformation. International Journal of Plasticity 2009;25:26.

[5] Zhou C, Biner S, LeSar R. Simulations of the effect of surface coatings on plasticity at small scales. Scripta Materialia 2010;63:1096.

[6] El-Awady JA, Wen M, Ghoniem NM. The role of the weakest-link mechanism in controlling the plasticity of micropillars. Journal of the Mechanics and Physics of Solids 2009;57:32.

[7] Quek SS, Zhang YW, Xiang Y, Srolovitz DJ. Dislocation cross-slip in heteroepitaxial multilayer films. Acta Materialia 2010;58:226.

[8] Zhou C, Biner SB, LeSar R. Discrete dislocation dynamics simulations of plasticity at small scales. Acta Materialia 2010;58:1565.

[9] Canova G, Kubin LP. Dislocation microstructures and plastic-flow - a 3-dimensional simulation. In: Maugin GA, editor. Continuum Models and Discrete Systems, Vol 2. Essex: Longman Scientific \& Technical, 1991. p.93.

[10] Kubin LP, Canova G. The modelling of dislocation patterns. Scripta Metallurgica et Materialia 1992;27:957.

[11] Verdier M, Fivel M, Groma I. Mesoscopic scale simulation of dislocation dynamics in fcc metals: Principles and applications. Modelling and Simulation in Materials Science and Engineering 1998;6:755.

[12] Clark WAT, Wagoner RH, Shen ZY, Lee TC, Robertson IM, Birnbaum HK. On the criteria for slip transmission across interfaces in polycrystals. Scripta Metallurgica Et Materialia 1992;26:203.

[13] Lee T, Robertson I, Birnbaum H. An In Situ transmission electron microscope 
deformation study of the slip transfer mechanisms in metals Metallurgical and Materials Transactions A 1990;21:2437.

[14] Lee TC, Robertson IM, Birnbaum HK. Prediction of slip transfer mechanisms across grain-boundaries. Scripta Metallurgica 1989;23:799.

[15] Shen Z, Wagoner RH, Clark WAT. Dislocation and grain-boundary interactions in metals. Acta Metallurgica 1988;36:3231.

[16] de Koning M, Kurtz RJ, Bulatov VV, Henager CH, Hoagland RG, Cai W, Nomura M. Modeling of dislocation-grain boundary interactions in FCC metals. Journal of Nuclear Materials 2003;323:281.

[17] de Koning M, Miller R, Bulatov VV, Abraham FF. Modelling grain-boundary resistance in intergranular dislocation slip transmission. Philosophical Magazine a-Physics of Condensed Matter Structure Defects and Mechanical Properties 2002;82:2511.

[18] Kumar R, Szekely F, Van der Giessen E. Modelling dislocation transmission across tilt grain boundaries in 2D. Computational Materials Science 2010;49:46.

[19] Xiang Y, Vlassak JJ. Bauschinger and size effects in thin-film plasticity. Acta Materialia 2006;54:5449.

[20] Espinosa HD, Berbenni S, Panico M, Schwarz KW. An interpretation of size-scale plasticity in geometrically confined systems. Proceedings of the National Academy of Sciences of the United States of America 2005;102:16933.

[21] Espinosa HD, Panico M, Berbenni S, Schwarz KW. Discrete dislocation dynamics simulations to interpret plasticity size and surface effects in freestanding FCC thin films. International Journal of Plasticity 2006;22:2091.

[22] von Blanckenhagen B, Arst E, Gumbsch P. Discrete dislocation simulation of plastic deformation in metal thin films. Acta Materialia 2004;52:773.

[23] Espinosa HD, Prorok BC, Peng B. Plasticity size effects in free-standing submicron polycrystalline FCC films subjected to pure tension. Journal of the Mechanics and Physics of Solids 2004;52:667.

[24] Hommel M, Kraft O. Deformation behavior of thin copper films on deformable substrates. Acta Materialia 2001;49:3935.

[25] Hall EO. The deformation and ageing of mild steel .3. discussion of results. Proceedings of the Physical Society of London Section B 1951;64:747. 
[26] Petch NJ. The cleavage strength of polycrystals. Journal of the Iron and Steel Institute 1953;174:25.

[27] Doerner MF, Nix WD. Stresses and deformation processes in thin-films on substrates. Critical Reviews in Solid State and Materials Sciences 1988;14:225.

[28] Nix WD. Mechanical-properties of thin-films. Metallurgical Transactions a-Physical Metallurgy and Materials Science 1989;20:2217.

[29] Venkatraman R, Bravman J, Nix W, Avies P, Flinn P, Fraser D. Mechanical properties and microstructural characterization of $\mathrm{Al}-0.5 \% \mathrm{Cu}$ thin films. Journal of Electronic Materials 1990;19:1231.

[30] Venkatraman R, Bravman JC. Separation of film thickness and grain-boundary strengthening effects in al thin-films on si. Journal of Materials Research 1992;7:2040.

[31] Chauhan S, Bastawros AF. Probing thickness-dependent dislocation storage in freestanding $\mathrm{Cu}$ films using residual electrical resistivity. Applied Physics Letters 2008;93.

[32] Fleck NA, Muller GM, Ashby MF, Hutchinson JW. Strain gradient plasticity: Theory and experiment. Acta Metallurgica et Materialia 1994;42:475.

[33] Hutchinson JW. Plasticity at the micron scale. International Journal of Solids and Structures 2000;37:225.

[34] Stölken JS, Evans AG. A microbend test method for measuring the plasticity length scale. Acta Materialia 1998;46:5109.

[35] Gruber PA, Bohm J, Onuseit F, Wanner A, Spolenak R, Arzt E. Size effects on yield strength and strain hardening for ultra-thin $\mathrm{Cu}$ films with and without passivation: A study by synchrotron and bulge test techniques. Acta Materialia 2008;56:2318.

[36] Haque MA, Saif MTA. Deformation mechanisms in free-standing nanoscale thin films: A quantitative in situ transmission electron microscope study. Proceedings of the National Academy of Sciences of the United States of America 2004;101:6335.

[37] Haque MA, Saif MTA. In situ tensile testing of nanoscale freestanding thin films inside a transmission electron microscope. Journal of Materials Research 2005;20:1769.

[38] Rajagopalan J, Han JH, Saif MTA. Plastic deformation recovery in freestanding nanocrystalline aluminum and gold thin films. Science 2007;315:1831.

[39] Chen MW, Ma E, Hemker KJ, Sheng HW, Wang YM, Cheng XM. Deformation twinning in nanocrystalline aluminum. Science 2003;300:1275. 
[40] Kumar KS, Suresh S, Chisholm MF, Horton JA, Wang P. Deformation of electrodeposited nanocrystalline nickel. Acta Materialia 2003;51:387.

[41] Eiper E, Keckes J, Martinschitz KJ, Zizak I, Cabié M, Dehm G. Size-independent stresses in $\mathrm{Al}$ thin films thermally strained down to $-100{ }^{\circ} \mathrm{C}$. Acta Materialia 2007;55:1941.

[42] Gruber PA, Solenthaler C, Arzt E, Spolenak R. Strong single-crystalline Au films tested by a new synchrotron technique. Acta Materialia 2008;56:1876.

[43] Oh SH, Legros M, Kiener D, Gruber P, Dehm G. In situ TEM straining of single crystal $\mathrm{Au}$ films on polyimide: Change of deformation mechanisms at the nanoscale. Acta Materialia 2007;55:5558.

[44] Thompson CV. The yield stress of polycrystalline thin-films. Journal of Materials Research 1993;8:237.

[45] Arzt E, Dehm G, Gumbsch P, Kraft O, Weiss D. Interface controlled plasticity in metals: dispersion hardening and thin film deformation. Progress in Materials Science $2001 ; 46: 283$.

[46] von Blanckenhagen B, Gumbsch P, Arzt E. Dislocation sources in discrete dislocation simulations of thin-film plasticity and the Hall-Petch relation. Modelling and Simulation in Materials Science and Engineering 2001;9:157.

[47] von Blanckenhagen B, Gumbsch P, Arzt E. Dislocation sources and the flow stress of polycrystalline thin metal films. Philosophical Magazine Letters 2003;83:1.

[48] Legros M, Cabie M, Gianola DS. In Situ Deformation of Thin Films on Substrates. Microscopy Research and Technique 2009;72:270.

[49] Rao SI, Dimiduk DM, Tang M, Parthasarathy TA, Uchic MD, Woodward C. Estimating the strength of single-ended dislocation sources in micron-sized single crystals. Philosophical Magazine 2007;87:4777.

[50] Kubin L, Devincre B, Hoc T. Modeling dislocation storage rates and mean free paths in face-centered cubic crystals. Acta Materialia 2008;56:6040.

[51] Parthasarathy TA, Rao SI, Dimiduk DM, Uchic MD, Trinkle DR. Contribution to size effect of yield strength from the stochastics of dislocation source lengths in finite samples. Scripta Materialia 2007;56:313.

[52] Derek Hull, Bacon DJ. Introduction to Dislocations, Fourth Edition. Butterworth-Heinemann, 2001. 


\section{CHAPTER 8}

\section{DISLOCATION DYNAMICS SIMULATIONS OF BAUSCHINGER EFFECTS IN METALLIC THIN FILMS}

The Bauschinger effect normally refers the decrease of reversal strength of a metal after a forward deformation [1-2]. It is an important phenomenon found in most crystalline materials. In single crystals, it is controlled by the reversibility of the accumulated dislocations during forward loading and the associated misorientation patterns [3-5]. In polycrystals, it is mainly attributed to dissolution of dislocation cell walls or sub-boundaries formed during pre-straining [6-8]. In precipitation-strengthened materials, the large $\mathrm{BE}$ is related to the dislocation interaction with precipitates that impede dislocations glide in traction and promote it under reversed loading [9-11]. Normally, all Bauschinger effect in bulk materials just appears during the reversed loading. Recently, an anomalous Bauschinger effect has been observed in metallic thin films with passivation layers [12-14], that the reverse flow already takes place on unloading. This $\mathrm{BE}$ is much stronger than in bulk materials, and indicates the reduced length scales plays a particular role on mechanical behavior of small scale materials during strain-path changes. According to the strain-gradient plasticity theory, the size-dependent plasticity at small scales results from the presence of plastic strain gradients that increases the resistance to plastic flow by locally increasing the dislocation density [15-18]. For the thin films with passivating layers, dislocations are prevented from exiting the film and the yield stress increases with decreasing film thickness can be explained by a plastic strain gradient near the film-passivation interface. However, the BE observed in passivated thin films cannot be predicted by strain gradient plasticity calculations [13].

As mechanical structures and devices are being created on a dimension comparable to

the length scales of the underlying dislocation microstructures, the dynamical behavior of 
discrete dislocations casts a significant impact on the plastic deformation of metallic materials at micron and submicron scales [19-20]. A detailed understanding of dislocation motion, multiplication and interactions in a confined geometry is the key to explain the plastic deformation of polycrystalline thin films. In this study, 3-D DD simulations have been used to investigate the Bauschinger effect in freestanding and passivated metallic thin films.

\subsection{Simulation procedures}

The 3D DD SIMULATIONS framework described in [21-23] has been used in our study to simulate the plasticity in $\mathrm{Cu}$ (FCC) polycrystalline thin films. In this work, the materials properties of $\mathrm{Cu}$ are used: shear modulus $\mu=50 \mathrm{GPa}$, Poisson's ratio $v=0.34$, and lattice constant $\mathrm{a}=0.36 \mathrm{~nm}$. In agreement with the experimental observations [13], the passivation layer on films was considered as an impenetrable obstacle for dislocations, while free surfaces both served as a sink for dislocations and also generated image forces and were modeled using the boundary element method (BEM) [24-25]. Finally, a sophisticated thermally-activated cross-slip model developed by Kubin and co-workers [26-27] was adopted in our DD simulations with Monte Carlo sampling to determine the activation of cross slip.

In this study, a volume element consisting of nine $(=3 \times 3 \times 1)$ columnar grains is set up and represents freestanding polycrystalline thin films. Each grain has the same size and is set in [100] directions. The cross-section of each grain is square, and the length of each side of the square represents grain sizes $(D)$ while the height of each grain is equal to the thickness of thin films $(H)$. Six sides of the grain aggregate are set as impenetrable obstacle for dislocations for passivated films and free surfaces from which dislocations can escape for freestanding films. All grain boundaries are considered as pure tilt boundary with the misorientation between adjacent grains less than $10^{\circ}$. In this case, the GB transmission strength, $\tau_{G B}$, compared with the critical stress to activate the Frank-Read source, $\tau_{F R}$, is in the 
range $\tau_{G B} / \tau_{F R} \approx 2, \ldots, 10$ [28-29]. When the resolved shear stress at the GB dislocation exceeds the GB transmission strength, the GB dislocation will transmit the grain boundary and continue operate in the outgoing grain. In our previous simulations [30], we found when $\tau_{G B} / \tau_{F R} \approx 5$, the computed and experimental stress-strain curves agree quite well. Thus, we use the same GB transmission strength in current simulations.

At the beginning of simulations, each grain contains a set of Frank-Read sources with random lengths on twelve $<011>\{111\}$ slip systems. All initial dislocation densities of following simulations are set around $1.0 \times 10^{13} \mathrm{~m}^{-2}$. In our simulations, tensile loading was applied on the grain aggregate in [100] direction with a constant strain rate equal to $2000 \mathrm{~s}^{-1}$. In order to mimic the plastic deformation in real polycrystalline thin films, we tracked the stress-strain evolution in the center grain of the aggregate and averaged the simulation results from ten simulation results from different initial dislocation configurations at the same dislocation density.

At the beginning of simulations, each grain contains a set of Frank-Read sources with random lengths on twelve $<011>\{111\}$ slip systems. All initial dislocation densities of following simulations are set around $1.0 \times 10^{13} \mathrm{~m}^{-2}$. In our simulations, tensile loading was applied on the grain aggregate in [100] direction with a constant strain rate equal to $2000 \mathrm{~s}^{-1}$. In order to mimic the plastic deformation in real polycrystalline thin films, we tracked the stress-strain evolution in the center grain of the aggregate and averaged the simulation results from ten simulation results from different initial dislocation configurations at the same dislocation density.

\subsection{Effect of passivation layers on the film strength}

To compare simulation results from freestanding films and passivated films, Figure 8.1a plots the stress-strain curves for both cases together. In our simulations, we fixed the grain size at $500 \mathrm{~nm}$, and varied the film thickness from 250 to $1000 \mathrm{~nm}$ with corresponding aspect 
ratios at $0.5,1.0$ and 2.0 . The $0.2 \%$ offset yield strength, denoted by $\sigma_{y}$, is obtained from the intercept of the dash dotted line in Figure 8.1a. The size-dependent behavior, in which thinner films have higher strength, is observed in both freestanding and passivated films. The stress-strain curves for freestanding films exhibited plateaus regime after yielding and
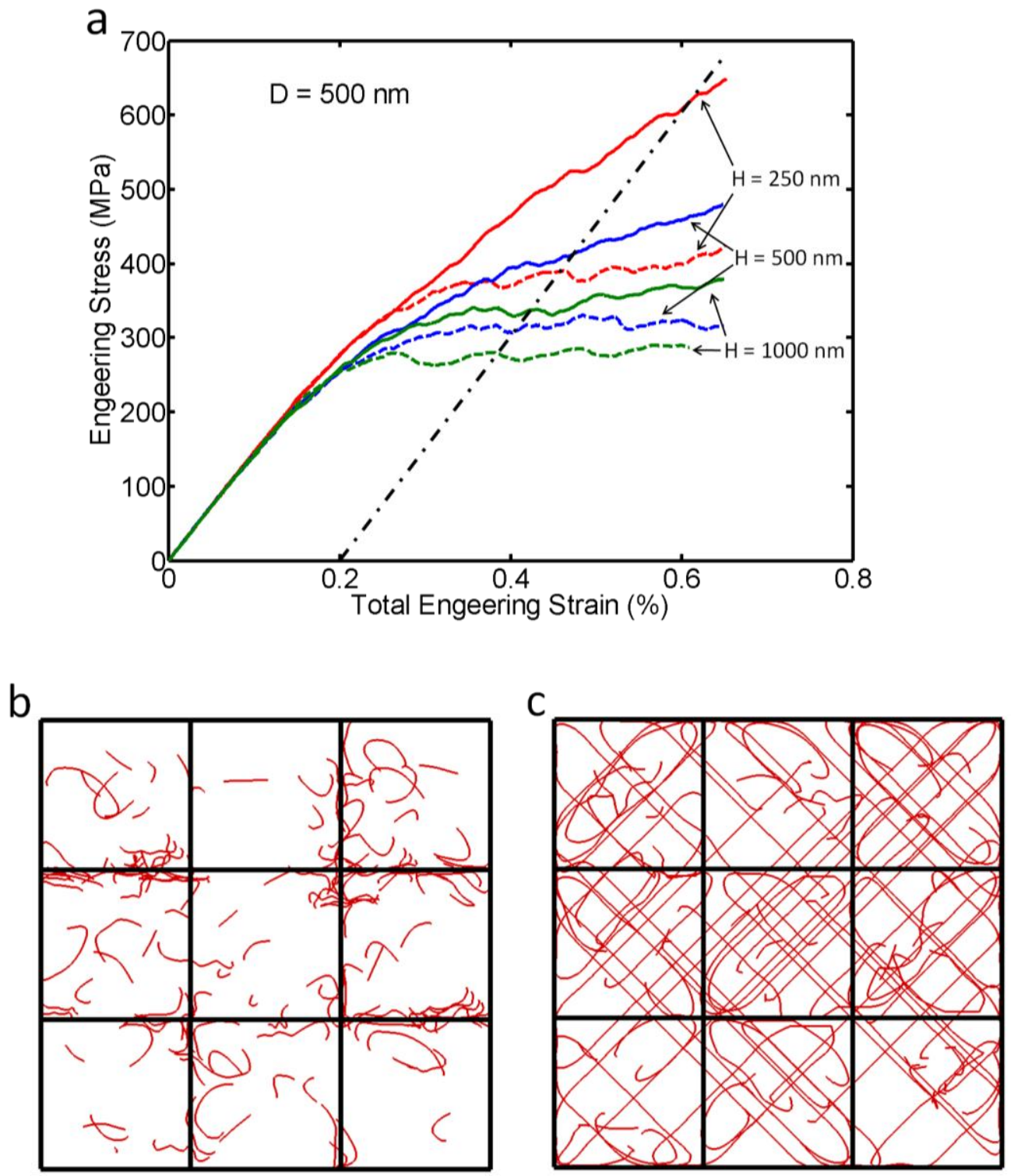

Figure 8.1 (a) Stress-strain curves of freestanding and passivated films under forward loading (dashed and solid lines for freestanding and passivated films, respectively); (b) dislocation structures in the $250 \mathrm{~nm}$ freestanding film; (c) dislocation structures in the $250 \mathrm{~nm}$ passivated film. 
the strain hardening rates approached zero in all thicknesses. While the stress-strain curves for passivated films had larger-strain-hardening rates than those for freestanding films. Furthermore, the strain-hardening rate in passivated films increases with decreasing film thickness that 250 and $1000 \mathrm{~nm}$ films have the highest and lowest hardening rates, respectively. Compared with freestanding films, the yield strengths for passivated films have approximately been increased by 13\%, 30\% and 56\% for thicknesses at 1000, 500 and 250 $\mathrm{nm}$, respectively. The typical dislocation structures in freestanding and passivated films after yielding are shown in Figure $8.1 \mathrm{~b}$ and c. Since dislocation sources can escape from the free surface without resistance, the dislocation structure in freestanding films is relative clean and the dislocations are composed of short segments truncated by free surfaces. In the passivated film shown in Figure 8.1c, lots of long misfit dislocations deposited at the interface between the film and passivation layers that results in higher dislocation density stored in the film. The misfit dislocations in passivated film produced a back stress to the on subsequently activated sources, caused dislocation pile-ups near the interface, and thus reduced the free path of mobile dislocations in the film and induced hardening with increasing strain. This can explain why passivated films exhibited higher hardening rate and strength than freestanding films.

\subsection{Effect of passivation layers on reverse plasticity of thin films}

In order to understand to effect of passivation layers on reverse plasticity of polycrystalline thin films, we unloaded the freestanding and passivated films from the same strain level to see the different responses between them. Figure 8.2a shows the response of freestanding and passivated films under unloading from pre-strains of $0.6 \%$ and $0.9 \%$. It is clear that unloading curves are nearly elastic and without any reverse plastic flow in freestanding films and only the unloading curve from pre-strain at $0.9 \%$ slightly deviates from elastic curve. On the other hand, the passivated film shows a significant Bauschinger 

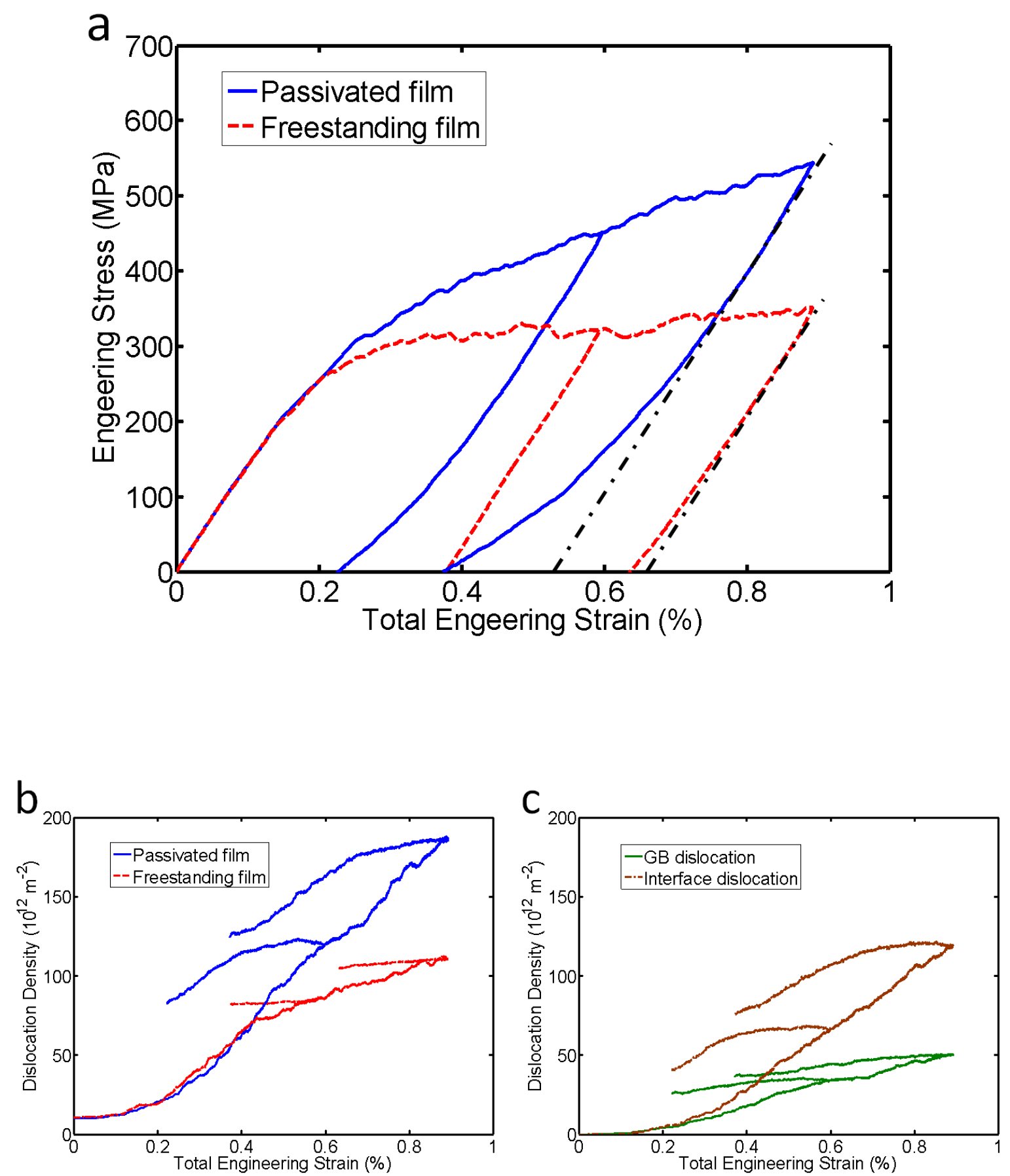

Figure 8.2 (a) Stress-strain curves of freestanding and passivated films during unloading (H and D are both equal to 500nm); (b) the corresponding total dislocation density evolution in both cases; (c) the corresponding grain boundary dislocation density evolution and interface dislocation density evolution in the passivated film. 
effect during unloading that large plastic flow occurs. In addition, the reverse plastic strain increases with increasing pre-strains as shown in Figure 8.2a. At this point, our simulation results agree well with experiment results that passivated films demonstrated a significant Bauschinger effect during unloading in contrast with freestanding films [13]. Figure 8.2b plots the total dislocation evolutions in both cases. We can see the dislocation increasing rate is higher in the passivated film than in the freestanding film, because the passivation layers can block dislocations near interfaces while free surface will assistant mobile dislocations escaping from the sample. During unloading, the dislocation density almost keeps constant in the freestanding films, while the density drops a lot with reverse strain in passivated films. However, there is not a direct relationship between the total dislocation density and the Bauschinger effect in thin films, since the passivated film exhibits much stronger Bauschinger effect than freestanding films, even they hold the same total dislocation densities. As shown in Figure 8.2b, total dislocation density in passviated film at $0.6 \%$ pre-strain is at the same level as that in passviated film at $0.9 \%$ pre-strain, but the mechanical response under unloading are totally different between these two cases shown in Figure 8.2a. To further analysis the Bauschinger effect in passivated films, the evolution of interface dislocation density and grain boundary (GB) dislocation density in passivated films have been plotted in Figure 8.2c. It is obvious that GB dislocation density did not change too much during unloading, while the interface dislocation density decreased fast with increasing reverse strain and has the same pace with the evolution of total dislocation density. After comparing the interface dislocation density curve in Figure 8.2c to the total dislocation density curve in Figure $8.2 \mathrm{~b}$, we can easily find the loss amount of total dislocation in passivated films approximates the loss amount of interface dislocation. In our simulations, the loss of interface dislocation results from the reversed motion of pile-up dislocations and collapse of misfit dislocations. Figure 8.3 illustrates one typical example of the reversed motion of pile-up dislocations. During forward loading, the misfit dislocations deposited at the interface between films and passivation layers produced a back stress on subsequently 

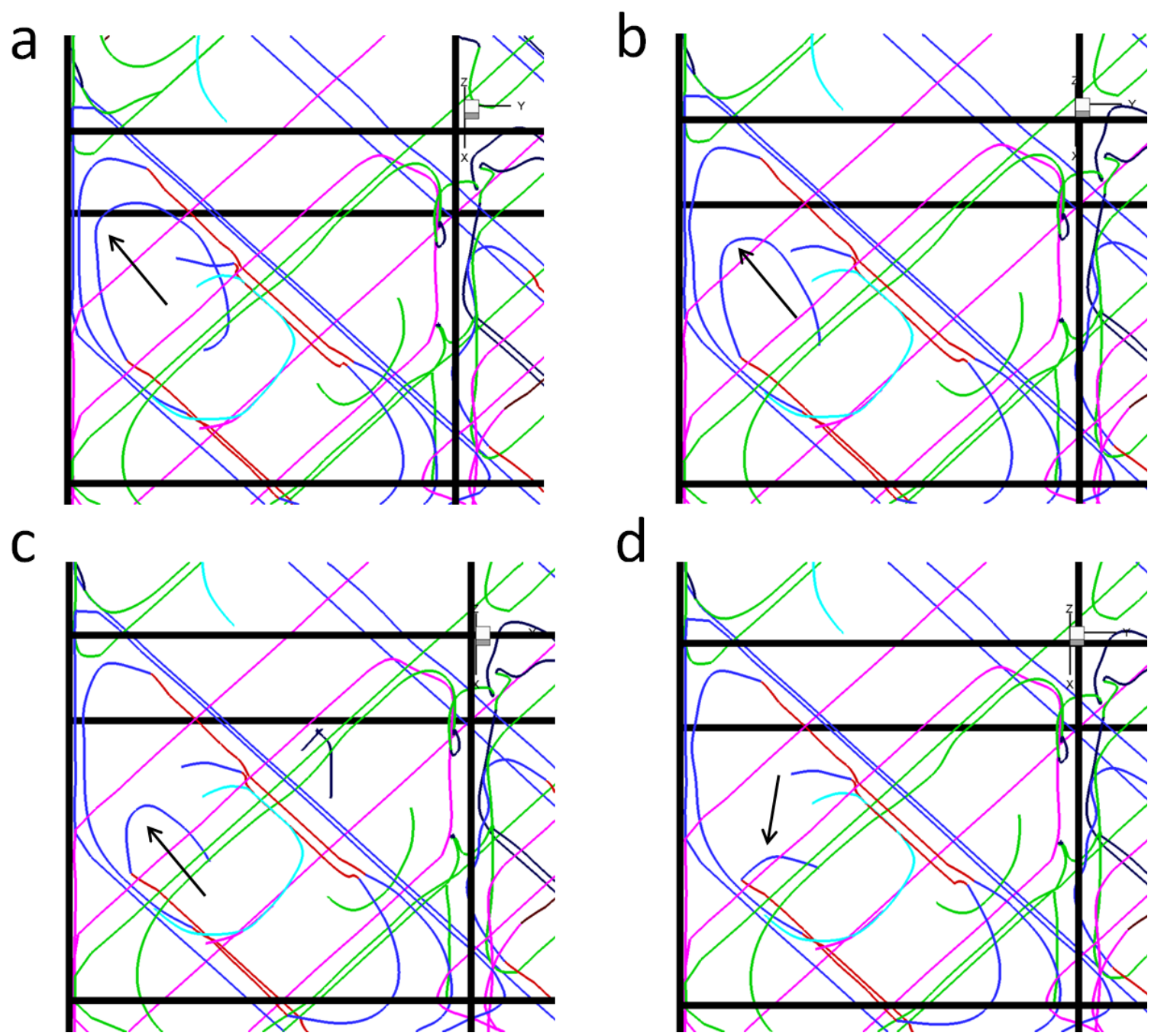

Figure 8.3 Illustration of the reversed motion of the pile-up dislocation (marked with arrow) in passivated films during unloading.

coming dislocations. When the following dislocation approaches the misfit dislocations, it will stop near interfaces due to the back stress. Although cross-slip of screw dislocations can relax part of these back-stresses from deposit misfit dislocation [31], there still lots of non-screw dislocation pile-ups formed near the interface. The total force on these immobile pile-up dislocations equals to zero and is composed of three major parts, the applied force, repulsive force, and self-force. The first part makes the dislocation bow-out and moving forward, while the other two parts make it moving back. When the applied load decreases, 
the immobile pile-up dislocations will move back to balance the three forces on them. With progress of unloading, the expanded dislocation continued shrinking and finally arrived at an equilibrium position even the whole film was still under tension as shown in Figure 8.3. If there is few dislocation pile-ups formed near misfit dislocations, the deposited long misfit will collapse from the interface and reverse move to its original position. The back motion of dislocations will create reverse plastic strain that results in the observed reverse plastic flow during unloading. Furthermore, the reverse plastic flow increases with the interface dislocation density as show in Figure 8.2, because more interface dislocations can have more sources to create reverse plastic strain during unloading. Although dislocation pile-ups may form near grain boundary, the number is limited as shown in Figure 8.1b and c that cannot generate sufficient reverse plastic flow during unloading. This can explain why current simulations and previous experiments [12-14] did not observed Bauschinger effect in freestanding films during unloading. Since the interface dislocation is the key factor to the Bauschinger effect in thin films, the freestanding cases are not considered in the following investigations and the analysis only focus passivated films.

\subsection{Bauschinger effect in passivated thin films}

To study the Bauschinger effect in passivated films quantitatively, we performed our simulations on films with different aspect ratios and unloaded them from different pre-strains. The Bauschinger strain and pre-strain are defined in Figure 8.4a and the normalized Bauschinger strain is plotted as a function of normalized pre-strain in Figure 8.4b. It is easy to see that the increasing pre-strain will promote the Bauschinger effect in passivated films, since the interface dislocation density increases with pre-strain as show in Figure 8.2c. Moreover, the film aspect ratio has a strong effect on the reverse flow in the passivated films, as the amplitude of BE strain increases faster in films with lower aspect ratios as shown in Figure 8.4. That because films with lower aspect ratios have larger interface areas that can 

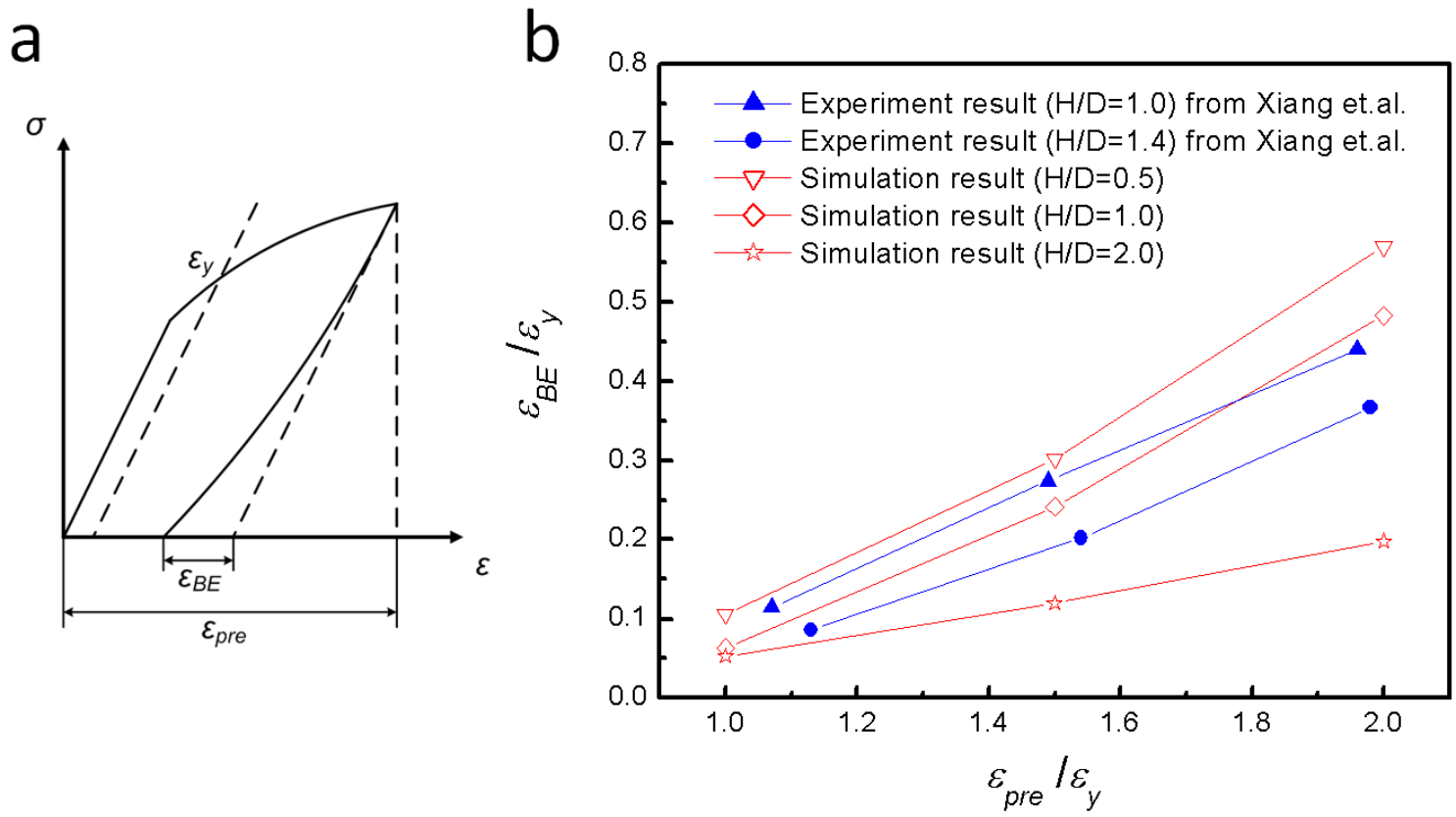

Figure 8.4 (a) Description of notations used for quantifying BE, $\varepsilon_{y}$ denotes yield strain, $\varepsilon_{\text {pre }}$ denotes pre- strain and $\varepsilon_{B E}$ denotes $\mathrm{BE}$ strain; (b) plot of normalized $\mathrm{BE}$ strain vs normalized pre-strain from simulation results on passivated films with different aspect ratios and comparison with experiment results from ref. [13].

adopt more misfit dislocations at the interface and more pile-ups dislocations near interfaces, finally result in larger reverse plastic flow during unloading. From Figure 8.4, we can see our simulation results march well with experiment results from ref. [13].

In bulk polycrystalline metals, Bauschinger effect is a widely observed and regarded as an intrinsic feature of the hardening process [3]. The Bauschinger effect in passivated thin films is also closely related to the strain hardening during forward loading, that low aspect ratio films have higher strain hardening rates as showed in Figure 8.1a and also exhibit stronger Bauschinger effect in Figure 8.4. However, the reverse plastic strain in bulk metals at the end of each unloading cycle is no more than $4 \%$ of maximum elastic strain [3]. While the Bauschinger strain in thin films can approach over $50 \%$ of the yield strain as shown in Figure $8.4 \mathrm{~b}$. This deference is caused by different hardening process in bulk metal and passivated film. The strain hardening in bulk metals normally results from dislocation 
reactions forming cell structures or subgrains [4, 6]. In passivated thin films, dislocation pile-ups are the main factor for the hardening process with increasing strain. Since dislocation pile-ups are more unstable compared with dislocation cell structures during unloading, the Bauschinger effect is much stronger in passivated thin films than in bulk metals at unloading.

\subsection{Conclusions}

The purpose of the present study is to enhance the understanding of the BE in small scale materials with the help of DD simulations, which account for the discrete nature of plasticity. In our simulations, we found passivated films have higher strain hardening rate and strength compared with freestanding films and the strain hardening rate in passivated films increases with decreasing film aspect ratios. Under unloading, passivated films exhibited a significant Bauschinger effect from pre-strains and the increasing pre-strain will promote more reverse plastic strain. Besides that, the amplitude of BE strain increases with decreasing film aspect ratios. The reverse motion of pile-up dislocation and collapse of misfit dislocations are responsible to the observed Bauschinger effect in passivated films. 


\section{References}

[1] Atkinson JD, Brown LM, Stobbs WM. Work-hardening of copper-silica .4. bauschinger effect and plastic relaxation. Philosophical Magazine 1974;30:1247.

[2] Pedersen OB, Brown LM, Stobbs WM. The bauschinger effect in copper. Acta Metallurgica 1981;29:1843.

[3] Buckley SN, Entwistle KM. The bauschinger effect in super-pure aluminum single crystals and polycrystals. Acta Metallurgica 1956;4:352.

[4] Hasegawa T, Yakou T, Kocks UF. Forward and reverse rearrangements of dislocations in tangled walls. Materials Science and Engineering 1986;81:189.

[5] Demir E, Raabe D. Mechanical and microstructural single-crystal Bauschinger effects: Observation of reversible plasticity in copper during bending. Acta Materialia 2010;58:6055.

[6] Hasegawa T, Yakou T, Karashima S. Deformation behavior and dislocation-structures upon stress reversal in polycrystalline aluminum. Materials Science and Engineering 1975;20:267.

[7] Stout M, Rollett A. Large-strain Bauschinger effects in fcc metals and alloys. Metallurgical and Materials Transactions A 1990;21:3201.

[8] Kocks UF, Mecking H. Physics and phenomenology of strain hardening: the FCC case. Progress in Materials Science 2003;48:171.

[9] Abel A, Ham RK. Cyclic strain behaviour of crystals of aluminum-4wt percent copper .i. bauschinger effect. Acta Metallurgica 1966;14:1489.

[10] Brown LM. Orowans explanation of bauschinger effect. Scripta Metallurgica 1977;11:127.

[11] Brown LM, Stobbs WM. Work-hardening of copper-silica .1. model based on internal stresses, with no plastic relaxation. Philosophical Magazine 1971;23:1185.

[12] Baker SP, Keller-Flaig RM, Shu JB. Bauschinger effect and anomalous thermomechanical deformation induced by oxygen in passivated thin $\mathrm{Cu}$ films on substrates. Acta Materialia 2003;51:3019.

[13] Xiang Y, Vlassak JJ. Bauschinger and size effects in thin-film plasticity. Acta Materialia 2006;54:5449.

[14] Xiang Y, Vlassak JJ. Bauschinger effect in thin metal films. Scripta Materialia 
2005;53:177.

[15] Fleck NA, Muller GM, Ashby MF, Hutchinson JW. Strain gradient plasticity: Theory and experiment. Acta Metallurgica et Materialia 1994;42:475.

[16] Hutchinson JW. Plasticity at the micron scale. International Journal of Solids and Structures 2000;37:225.

[17] Fredriksson P, Gudmundson P. Size-dependent yield strength of thin films. International Journal of Plasticity 2005;21:1834.

[18] Gudmundson P. A unified treatment of strain gradient plasticity. Journal of the Mechanics and Physics of Solids 2004;52:1379.

[19] Kraft O, Gruber PA, Mönig R, Weygand D. Plasticity in Confined Dimensions. Annual Review of Materials Research 2010;40:293.

[20] Uchic MD, Shade PA, Dimiduk DM. Plasticity of Micrometer-Scale Single Crystals in Compression. Annual Review of Materials Research 2009;39:361.

[21] Ghoniem NM, Sun LZ. Fast-sum method for the elastic field off three-dimensional dislocation ensembles. Physical Review B 1999;60:128.

[22] Ghoniem NM, Tong SH, Sun LZ. Parametric dislocation dynamics: A thermodynamics-based approach to investigations of mesoscopic plastic deformation. Physical Review B 2000;61:913.

[23] Wang ZQ, Ghoniem N, Swaminarayan S, Lesar R. A parallel algorithm for 3D dislocation dynamics. Journal of Computational Physics 2006;219:608.

[24] Becker A. The Boundary Element Method in Engineering: A Complete Course McGraw-Hill International, 1992.

[25] El-Awady JA, Biner SB, Ghoniem NM. A self-consistent boundary element, parametric dislocation dynamics formulation of plastic flow in finite volumes. Journal of the Mechanics and Physics of Solids 2008;56:2019.

[26] Canova G, Kubin LP. Dislocation microstructures and plastic-flow - a 3-dimensional simulation. In: Maugin GA, editor. Continuum Models and Discrete Systems, Vol 2. Essex: Longman Scientific \& Technical, 1991. p.93.

[27] Kubin LP, Canova G. The modelling of dislocation patterns. Scripta Metallurgica et Materialia 1992;27:957.

[28] de Koning M, Miller R, Bulatov VV, Abraham FF. Modelling grain-boundary resistance in intergranular dislocation slip transmission. Philosophical Magazine 
a-Physics of Condensed Matter Structure Defects and Mechanical Properties 2002;82:2511.

[29] Kumar R, Szekely F, Van der Giessen E. Modelling dislocation transmission across tilt grain boundaries in 2D. Computational Materials Science 2010;49:46.

[30] Zhou C, LeSar R. Dislocation dynamics simulations of plasticity in polycrystalline thin films.In preperation to Acta Materialia.

[31] Zhou C, Biner S, LeSar R. Simulations of the effect of surface coatings on plasticity at small scales. Scripta Materialia 2010;63:1096. 


\section{CHAPTER 9 \\ CONCLUSIONS}

Miniaturization of structures and devices in micro- and nanotechnology leads to the development of materials and compounds with novel properties that cannot simply be extrapolated from material properties on larger scales. Mechanical behavior and reliability of devices containing metallic structures are of critical importance to innovations in integrated micro electronics, electro-mechanical, optoelectronic, and micro- or nano-electro-mechanical devices. DD simulations, in which the dislocations are the simulated entities, offer a way to extend length scales beyond those of atomistic simulations and the results from DD simulations can be directly compared with the micromechanical tests. In this research, 3-D DD simulations was used to study the plastic deformation of nano- and micro-scale materials and understand the correlation between dislocation motion, interactions and the mechanical response.

In Chapter 4, an experimental-like initial dislocation structures cut from larger deformed samples have been introduced into 3-D DD simulations to study the plasticity in small sizes. The results indicate that the loading direction has negligible effect on the flow stress with both multi-slip and single-slip loading resulting in the similar saturation. This lack of a dependence on loading direction can be easily understood. Since the number of dislocation sources decreases with the sample diameters, the probability to activate a source with low Schmid factors increases in small samples. In small samples, dynamic sources can be easily generated by cross-slip or collinear reactions, the stability of which depends on the position

and sample size. There were at least two origins of "exhaustion hardening": the escape of dynamic sources from the surface and dislocation interactions such as junction formation. Both of these effects shut off the activated sources, leading to the flow intermittency. The "mechanical annealing" at the early stage of deformation were seen to arise from the surface 
dislocations and the weakly-entangled dislocations leaving the sample. The drop in dislocation density was followed by an increase that always resulted from processes that were enabled by cross-slip. The scarcity of available dislocation sources gives a major contribution to the higher flow stress and larger scatter of strength in smaller sizes. The scaling law determined from the current simulation results is close to that found experimentally.

In Chapter 5, 3-D DD simulations were employed to study the dynamic behavior of internal dislocation sources in micropillars of different sizes. From the simulation results, we identified the dominating plastic deformation mechanisms at small scales by combining our modeling results. We note that these mechanisms are consistent with the available experimental data. In confined volumes, image stresses alter the local resolved shear stresses on slip planes, resulting in an increase in the probability of cross-slip to form new internal sources. These naturally formed sources have shorter residence lifetimes in smaller samples under the influence of attractive image forces from the nearby surfaces. The normalized critically resolved shear stress for a number of FCC single crystals exhibited a similar size-dependent behavior for all the materials. The generalized single-arm dislocation model can reasonably predict both the increase of yield strength with decreasing sample size, as well as the statistical variation of the strength at small scales. The plastic deformation of FCC single crystals at small scales depends not only on sample size but also on the dislocation density. At nano-and micro-scales, there is a critical size for dislocation starvation, which strongly depends on the initial dislocation density. Below this critical size, the dislocation loss rate will exceed the multiplication rate and thus nucleation of surface dislocations and dislocation starvation hardening will likely dominate plastic deformation process. Otherwise, multiplication of internal dislocation sources should control the plastic flow with increasing strain.

In Chapter 6, our simulations offer an explanation for the significant increase in compressive strength and formation of band structures in coated micropillars, demonstrating 
a fundamentally different strengthening mechanism in coated micropillars than in samples with free surfaces. In the coated samples, dislocations are blocked from leaving the sample, leading to dislocation pile-ups that induce a strong back stress on the later-activated sources, inhibiting further dislocation nucleation. Thus, the coated samples exhibited a higher strain-hardening rate, smaller strain bursts and greater flow stresses than those in samples with free surface. In addition, cross slip activated in coated samples enable screw dislocations to escape their original slip plane, generating more mobile dislocation sources for plastic deformation, and enabling the formation of banded structures and subcells.

In Chapter 7, a 3-D DD simulation was set up to investigate the plasticity of freestanding polycrystalline thin films. Both cross-slip and grain boundary relaxation mechanisms have been considered in our simulations, which are important in the plastic deformation of polycrystalline films and neglected by previous studies. According to our simulation results, a spiral source model has been established to predict the size-dependent strength in thin films. At a constant film thickness, the total dislocation density deceases with increasing grain sizes due to the increment of surface area to volume ratio. In films with pancake-like grains, the dependence of yield stress on the grain size gradually becomes weaker with decreasing film thickness. In contrast, the needle-like grains strength the grain boundary effect in polycrystalline films and grain size dependent behaviors in films with high aspect ratio can be described by Hall-Petch relation. With the same grain size, high aspect ratio films can hold higher total and GB dislocation density, since they have large grain boundary areas per volume in the film. The yield strength of films can scale proportionally to the reciprocal of thickness. With high aspect ratios increases, the dependence of the film strength on thickness becomes weaker due to the effect of free surfaces has been diminished. According to the dislocation structures observed in our simulations, a spiral source model has been set up to predict the yield stress of thin films. Comparison with data on freestanding thin films shows that spiral source model can reasonably explain the increase of strength with film thickness decreasing and the large scatter in the magnitude of strength in thinner films. 
In Chapter 8, the purpose is to enhance the understanding of the BE in small scale materials with the help of DD simulations, which account for the discrete nature of plasticity. In our simulations, we found passivated films have higher strain hardening rate and strength compared with freestanding films and the strain hardening rate in passivated films increases with decreasing film aspect ratios. Under unloading, passivated films exhibited a significant Bauschinger effect from pre-strains and the increasing pre-strain will promote more reverse plastic strain. Besides that, the amplitude of BE strain increases with decreasing film aspect ratios. The reverse motion of pile-up dislocation and collapse of misfit dislocations are responsible to the observed Bauschinger effect in passivated films.

Through a series of simulations, detailed investigation of the relationship between material microstructure and mechanical properties of small scale materials were performed by the method of dislocation dynamics. Numerical results can be directly compared with experiment results that indicate DD simulations are of great help in understanding plasticity at small scales. 